\title{
Alinhamentos e comparação de sequências
}

Francisco Eloi Soares de Araujo

TESE APRESENTADA

AO

Instituto DE MATEMÁtica e EstatísticA

DA

Universidade DE SÃo PAUlo

PARA

OBTENÇÃO DO TÍTULO

$\mathrm{DE}$

Doutor EM CiÊnCIAS

Programa: Ciência da Computação

Orientador: Prof. Dr. José Augusto Ramos Soares

Durante o desenvolvimento deste trabalho o autor recebeu auxílio financeiro da CAPES

São Paulo, 23 de julho de 2012 


\title{
Alinhamentos e comparação de sequências
}

\author{
Esta versão definitiva da tese \\ contém as correções e alterações sugeridas pela \\ Comissão Julgadora durante a defesa realizada \\ por Francisco Eloi Soares de Araujo em 24 de maio de 2012.
}

Comissão Julgadora:

- Prof. Dr. José Augusto Ramos Soares (Presidente) - IME - USP

- Prof. Dr. Carlos Eduardo Ferreira - IME - USP

- Prof. Dr. Luiz Carlos da Silva Rozante - CMCC - UFABC

- Prof. Dr. Zanoni Dias - IC - UNICAMP

- Prof. Dr. Fábio Henrique Viduani Martinez - FACOM - UFMS 


\section{Agradecimentos}

Muitos parentes e amigos foram importantes para o nosso doutorado. Certamente cada um desses sabe da sua importância nesse processo. Se eu esquecer de referenciar alguém direta ou indiretamente, perdoe-me pois a memória às vezes falha.

Agradeço inicialmente e principalmente à minha família: à Ione, minha esposa e aos meus filhos Gabriel, Sarah e Pedro que me deram amor e apoio logístico durante todos os anos de meu doutorado; e ao José Augusto, meu orientador e amigo, pelos ensinamentos e pela paciência sobrenatural. Certamente, sem o apoio da família e a paciência do José Augusto, esse trabalho não teria terminado.

Agradeço aos irmão e cunhados, sobrinhos e sobrinhas, sogro e sogra pelo apoio moral, em particular à minha cunhada Cláudia pelo incentivo e também pelas caronas do e para o IME. Agradeço também à minha família que estou redescobrindo em Campo Grande pela acolhida e pelo carinho recebido quando cheguei naquela cidade: meu tio Jair e meus primos Aily, Aise e Fernando, bem como os respectivos companheiros e descendentes.

Pela torcida e pela amizade, agradeço aos amigos do colégio São Luís; aos colegas de Jaboticabal incluindo em particular os irmãos de coração da república Amoribunda; aos amigos com quem trabalhei, Paulo Sérgio na Unisa, Hirata, Joyce, Nelson e Shirley no Senac, e Ana Lúcia, Cláudia Melo, Gabiru, Hamilton e Helena na Metodista; aos atuais amigos e colegas de trabalho da UFMS, em particular aos companheiros da FACOM-2 Carlos Higa, Fábio Iaione e Vagner Pedrotti que acompanharam os últimos momentos desse trabalho; aos amigos de doutorado Alexandre, Álvaro, Cao, Charlie Brown, Fábio Viduani, Jair Donadelli, Marco Aurélio, Mário Leston, Rogério e Said, e aos professores do IME Coelho, Cristina, José Augusto, Nami, Paulo Feofiloff, Yoshiharu e Yoshiko que me ensinaram muito. Também agradeço ao Professor Carlinhos do IME que esteve presente em todas as etapas de avaliação deste trabalho com sugestões motivadoras e enriquecedoras.

Agradeço ainda à CAPES pelo auxílio financeiro durante o doutorado.

Elói Araújo 
Aos meus pais (in memoriam), à minha esposa Ione e aos meus queridos filhos Gabriel, Sarah e Pedro, que sempre me apoiaram e ao meu orientador, a quem fico grato pela oportunidade de estudar computação. 


\section{Resumo}

Araujo, F. E. S. Alinhamentos e comparação de sequências. 2012. Tese (Doutorado) Instituto de Matemática e Estatística, Universidade de São Paulo, São Paulo, 2012.

A comparação de sequências finitas é uma ferramenta que é utilizada para a solução de problemas em várias áreas. Comparamos sequências inferindo quais são as operações de edição de substituição, inserção e remoção de símbolos que transformam uma sequência em uma outra. As matrizes de pontuação são estruturas largamente utilizadas e que definem um custo para cada tipo de operação de edição. Uma matriz de pontuação $\gamma$ é indexada pelos símbolos do alfabeto. A entrada de $\gamma$ na linha a, coluna b mede o custo da operação de edição para substituir o símbolo a pelo símbolo b. As matrizes de pontuação induzem funções que atribuem uma pontuação para um conjunto de operações de edição. Algumas dessas funções para a comparação de duas e de várias sequências são estudadas nesta tese.

Quando cada símbolo de cada sequência é editado exatamente uma vez para transformar uma sequência em outra, o conjunto de operações de edição pode ser representado por uma estrutura conhecida por alinhamento. Descrevemos uma estrutura para representar o conjunto de operações de edição que não pode ser representado por um alinhamento convencional e descrevemos um algoritmo para encontrar a pontuação de uma sequência ótima de operações de edição usando um algoritmo conhecido para encontrar a pontuação de um alinhamento convencional ótimo.

Considerando três diferentes funções induzidas de pontuação, caracterizamos, para cada uma delas, a classe das matrizes para as quais as funções induzidas de pontuação são métricas nas sequências.

Dadas duas matrizes de pontuação $\gamma$ e $\delta$, dizemos que elas são equivalentes para uma dada função que é induzida por uma matriz de pontuação e que avalia a qualidade de um alinhamento se, para quaisquer dois alinhamentos $A$ e $B$, vale o seguinte: o alinhamento $A$ é "melhor" do que o alinhamento $B$ considerando a matriz $\gamma$ se e somente se $A$ é "melhor" do que o alinhamento $B$ considerando a matriz $\delta$. Neste trabalho, determinamos condições necessárias e suficientes para que duas matrizes de pontuação sejam equivalentes.

Finalmente, definimos três novos critérios para pontuar alinhamentos de várias sequências. Todos os critérios consideram o comprimento do alinhamento além das operações de edição por ele representadas. Para cada um dos critérios definidos, propomos um algoritmo e o problema de decisão correspondente mostramos ser NP-completo. 
Palavras-chave: métrica, matrizes equivalentes, custo normalizado de alinhamentos, distância de edição, alinhamento estendido, alinhamento de sequências, alinhamento de várias sequências. 


\section{Abstract}

Araujo, F. E. S. Alignment and comparison of sequences. 2012. Tese (Doutorado) Instituto de Matemática e Estatística, Universidade de São Paulo, São Paulo, 2012.

Comparison of finite sequences is a tool used to solve problems in several areas. In order to compare sequences, we infer which are the edit operations of substitution, insertion and deletion of symbols that transform one sequence into another. Scoring matrices are a widely used structure to define a cost for each type of edit operation. A scoring matrix $\gamma$ is indexed by symbols of an alphabet. The entry in $\gamma$ in row $a$ and column $b$ measures the cost of the edit operation for replacing symbol a by symbol b. Scoring matrices induce functions that assign a score for a set of edit operations. Some of these functions for comparing two and multiple sequences are studied in this thesis.

If each symbol is edited exactly once for transforming a sequence into another, the set of edit operations can be represented by a structure called alignment. We describe a structure to represent the set of edit operations that cannot be represented by a conventional alignment and we design an algorithm to find the cost of an optimal sequence of edit operations by using a known algorithm to find the cost of an optimal alignment.

Considering three different kinds of induced scoring functions, we characterize, for each one of them, the class of matrices for which the induced scoring functions are metrics on sequences.

Given two scoring matrices $\gamma$ and $\delta$, we say they are equivalent for a given function that is induced by a scoring matrix and that evaluates the quality of an alignment if, for any two alignments $A$ and $B$ of two sequences, we have the following: alignment $A$ is "better" than $B$ considering scoring matrix $\gamma$ if and only if $A$ is "better" than $B$ considering scoring matrix $\delta$. In this work, we determine necessary and sufficient conditions for scoring matrices to be equivalent.

Finally, we define three new criteria for scoring alignments of several sequence. Every criterion considers the length of the alignment and the edit operations represented by it. An algorithm for each criterion is studied and the corresponding decision problem is shown to be NP-complete.

Keywords: metric, equivalent matrices, normalized alignment cost, edit distance, extended alignment, sequence alignment, multiple sequence alignments. 


\section{Sumário}

1 Introdução $\quad \mathbf{1}$

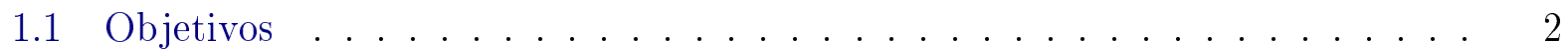

1.2 Contribuições . . . . . . . . . . . . . . . . . . . 5

1.3 Organização do Trabalho . . . . . . . . . . . . . . . . 5

$\begin{array}{lll}2 & \text { Conceitos } & 7\end{array}$

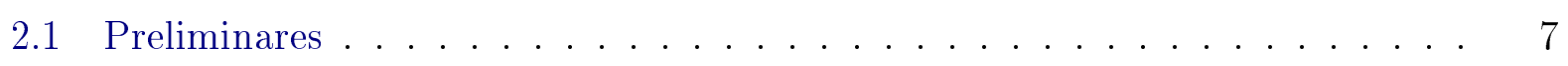

2.1.1 Alfabeto e sequências . . . . . . . . . . . . . . . . 7

2.1.2 Alinhamentos ....................... 7

2.1 .3 Matrizes de pontuação . . . . . . . . . . . . . . 8

2.1.4 Digrafos ............................ 9

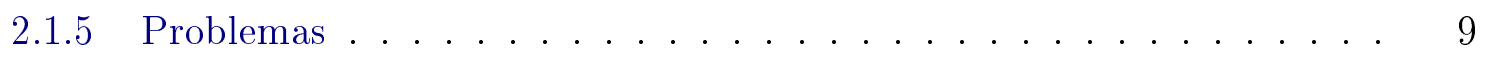

2.1.6 Fator de aproximação . . . . . . . . . . . . . . . . 10

2.2 Alinhamento de duas sequências . . . . . . . . . . . . . . . . 10

2.2 .1 Critério $v \mathrm{~A}_{\gamma} \ldots \ldots \ldots \ldots \ldots \ldots \ldots$

2.2 .2 Critério $v \mathrm{~N}_{\gamma} \ldots \ldots \ldots \ldots \ldots \ldots \ldots \ldots$

2.3 Alinhamento de várias sequências . . . . . . . . . . . . . . 28

2.3 .1 Algoritmos exatos . . . . . . . . . . . . . . . 29

2.3.2 Complexidade do problema . . . . . . . . . . . 30

$\begin{array}{lll}3 & \text { Alinhamento Estendido } & 37\end{array}$

4 Matrizes que induzem métricas $\quad 43$

4.1 Distância de alinhamento . . . . . . . . . . . . . . . . . 47

4.2 Distância normalizada de alinhamento . . . . . . . . . . . 55

4.3 Distância de edição . . . . . . . . . . . . . . . . . . 66

4.4 Comentário final . . . . . . . . . . . . . . . . 71

5 Matrizes equivalentes $\quad 73$

5.1 Operações $\otimes \mathrm{e} \oplus$ nas matrizes de pontuação . . . . . . . . . . . 74

5.2 Condições necessárias para $\gamma \sim \delta$ em $\mathbb{B} \ldots \ldots \ldots . \ldots 76$ 
6 Alinhamento de várias sequências $\quad 83$

6.1 Introdução . . . . . . . . . . . . . . . . . . 83

6.2 Algoritmos exatos . . . . . . . . . . . . . . . . . 84

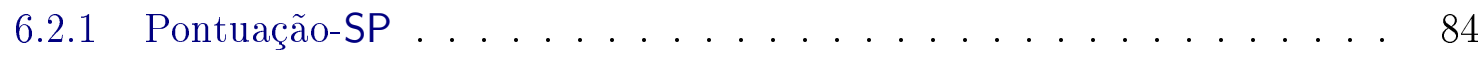

6.2 .2 Critério $\mathrm{V}_{\gamma}^{1} \ldots \ldots \ldots \ldots \ldots \ldots \ldots$

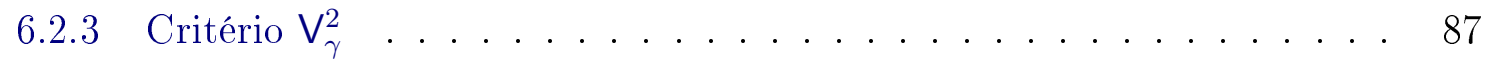

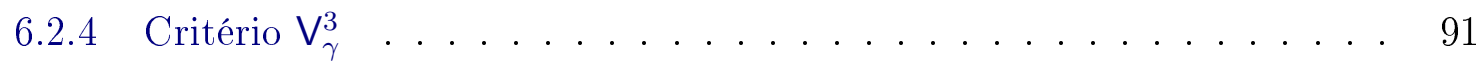

6.3 Complexidade . . . . . . . . . . . . . . . . . 92

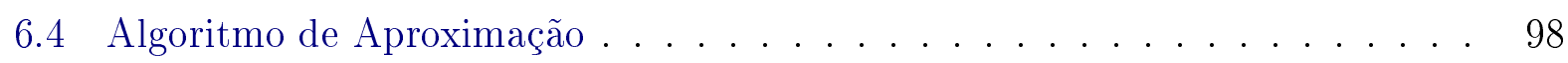

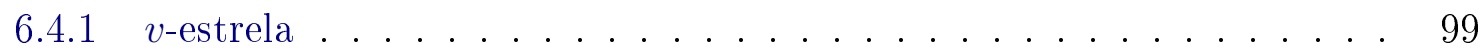

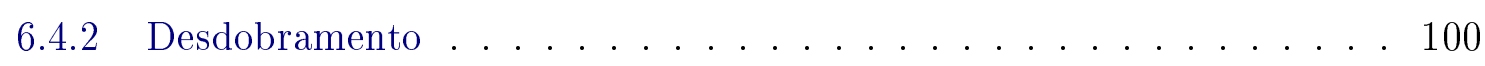

6.4 .3 Alinhamento compatível . . . . . . . . . . . . . . . . . 102

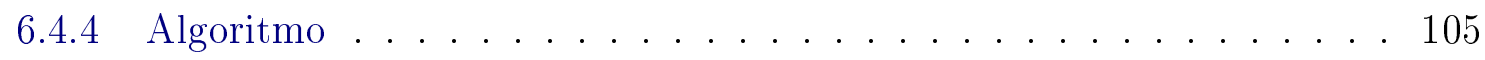

$\begin{array}{llr}7 & \text { Conclusão } & 109\end{array}$ 


\section{Capítulo 1}

\section{Introdução}

A comparação de sequências finitas é uma ferramenta que é utilizada para a solução de problemas em várias áreas tais como biologia computacional [SM97], processamento de textos [AG97], reconhecimento de padrões [MV93], etc. Uma maneira de comparar uma sequência com outras consiste em determinar quais são as operações de edição de inserção, remoção e substituição que transformam uma sequência em outras.

O alinhamento de sequências finitas é uma estrutura que permite a visualização de um conjunto de operações de edição que transformam uma sequência nas demais. Dependendo da aplicação e da formulação do problema, duas ou várias sequências podem ser comparadas. Comparamos duas sequências quando, por exemplo, queremos identificar se dois fragmentos de DNA possuem a mesma origem. Comparamos várias sequências quando, por exemplo, queremos representar regiões altamente conservadas em um conjunto de proteínas. Um alinhamento que representa a comparação de duas ou mais sequências é obtido colocando uma sequência embaixo da outra de modo que os símbolos "editados" fiquem em uma mesma coluna. Um símbolo especial $\sqcup$ é utilizado de modo a preencher espaços em branco quando alinhamos as sequências. Assim,

$$
\left[\begin{array}{ccccc}
a & b & c & c & \sqcup \\
a & \sqcup & b & c & b \\
& b & b & c & \sqcup
\end{array}\right],\left[\begin{array}{llll}
a & b & c & c \\
a & b & c & b \\
b & b & c & c
\end{array}\right] \text { e }\left[\begin{array}{ccccc}
a & b & c & \sqcup & c \\
\sqcup & a & b & c & b \\
b & b & \sqcup & b & c
\end{array}\right]
$$

são alinhamentos de (abcc, abcb, bbc).

Uma função objetivo é definida para atribuir uma pontuação a cada alinhamento. A função objetivo utilizada é o critério para pontuar um alinhamento, e um alinhamento de uma $k$-upla de sequências finitas $S$ cuja pontuação da função objetivo é mínima é dito alinhamento ótimo de $S$ para o critério considerado.

Seja $\mathcal{S}$ o conjunto de todas as $k$-uplas de sequências finitas. Dizemos que $f: \mathcal{S} \rightarrow \mathbb{R}$ é uma função ótima para um dado critério se $f(S)$ é a pontuação de um alinhamento ótimo de $S \in \mathcal{S}$.

Nesta tese, estudamos alguns critérios para pontuar alinhamentos de duas e de várias sequências. Estudamos também um critério para pontuar um conjunto de operações de edição que transforma uma sequência em outra mas, como veremos, não possui um alinhamento convencional para representá-lo. 


\section{$1.1 \quad$ Objetivos}

Seja $\Sigma$ um alfabeto e $\Sigma_{\sqcup}=\Sigma \cup\{\sqcup\}$, onde o símbolo $\sqcup \notin \Sigma$ é utilizado para representar inserções e remoções. Denotamos por $\Sigma^{*}$ o conjunto de todas as sequências finitas formadas com elementos de $\Sigma$.

Um alinhamento de duas sequências pode ser visto como uma representação de um conjunto de operações de edição de inserção, remoção e de substituição que transformam uma sequência em outra. Nesse sentido, o número de colunas é exatamente a quantidade dessas operações de edição. Por exemplo, o alinhamento

$$
\left[\begin{array}{lllll}
a & b & - & a & \sqcup \\
b & - & a & a & b
\end{array}\right]
$$

representa uma transformação de aba em baab através das substituições do primeiro e do último a de aba por b e a respectivamente. A remoção do b e a inserção apropriada de um a e um b em aba completam a transformação.

Duas sequências podem ser alinhadas de várias maneiras diferentes. Uma dada estrutura conhecida por matriz de pontuação pode ser usada para avaliar a qualidade de um alinhamento de duas sequências. Uma matriz de pontuação $\gamma$ para $\Sigma$ é uma matriz de números reais indexada nas linhas e nas colunas por elementos de $\Sigma_{\sqcup}$, ou seja, se a, b são símbolos em $\Sigma_{u}, \gamma_{a \rightarrow b}$ denota a entrada de $\gamma$ na linha a e coluna b. Se a, b $\in \Sigma$, então $\gamma_{a \rightarrow b}$ é o custo de substituiçãa do símbolo a pelo símbolo $\mathrm{b}, \gamma_{\mathrm{a} \rightarrow \sqcup}$ é o custo de remoção do símbolo a e $\gamma_{\hookrightarrow \rightarrow b}$ é o custo de inserção do símbolo b. As operações de substituição, inserção e remoção sobre uma sequência são chamadas de operações de edição. O valor de $\gamma_{{ }} \rightarrow_{\sqcup}$ é indefinido. A função $v \mathrm{~A}_{\gamma}$ associa a cada alinhamento $A$ a pontuação $v \mathrm{~A}_{\gamma}(A)$ que é a soma dos custos das operações de edição representadas em $A$. A função ótima para esse critério é opt $\mathrm{A}_{\gamma}$. A função opt $\mathrm{A}_{\gamma}$ é conhecida como distância de Levenshtein [Lev66] se, para cada par de elementos $\mathrm{a} \neq \mathrm{b}$ em $\Sigma_{\mathrm{b}}$, temos $\gamma_{\mathrm{a} \rightarrow \mathrm{a}}=0$ e $\gamma_{\mathrm{a} \rightarrow \mathrm{b}}=1$. Entretanto, nem sempre a função opt $\mathrm{A}_{\gamma}$ pode ser chamada de distância, ou seja, nem sempre optA $\mathrm{A}_{\gamma}$ é uma métrica em $\Sigma^{*}$. Sellers [Sel74] mostrou que se $\gamma$ é uma métrica em $\Sigma$, então opt $\mathrm{A}_{\gamma}$ também é uma métrica em $\Sigma^{*}$. Determinamos [AS06] as condições necessárias e suficientes da matriz $\gamma$ para que opt $\mathrm{A}_{\gamma}$ seja métrica em $\Sigma^{*}$. Mostramos, por exemplo, que se a matriz de pontuação é

$$
\gamma=\begin{array}{c|cccc} 
& \mathrm{a} & \mathrm{b} & \mathrm{c} & \mathrm{c} \\
\hline \mathrm{a} & 0 & 1 & 3 & 1 \\
\mathrm{~b} & 1 & 0 & 1 & 1 \\
\mathrm{c} & 4 & 1 & 0 & 1 \\
& 1 & 1 & 1 &
\end{array}
$$

então optA $\mathrm{A}_{\gamma}$ é uma métrica em $\Sigma^{*}$, embora $\gamma$ não seja métrica em $\Sigma_{\llcorner}$pois $\gamma_{a \rightarrow c} \neq \gamma_{c \rightarrow a}$ e $\gamma_{\mathrm{a} \rightarrow \mathrm{c}} \not \leq \gamma_{\mathrm{a} \rightarrow}+\gamma_{\mathrm{c}} \rightarrow \mathrm{c}$.

Navarro [Nav06] questionou se o resultado acima poderia ser estendido caracterizando assim, por exemplo, a classe de matrizes de pontuação $\gamma$ onde opt $\mathrm{A}_{\gamma}$ é uma quasimétrica em $\Sigma^{*}$. Como quasimétrica possui, exceto pela simetria, as propriedades de uma métrica, o questionamento de Navarro motivou a investigação individual, para cada uma das propriedades $P$ de uma métrica, das condições necessárias e suficientes de $\gamma$ para opt $\mathrm{A}_{\gamma}$ possuir a propriedade $P$ em $\Sigma^{*}$.

Se $\gamma$ não possui a propriedade da desigualdade triangular em $\Sigma_{\llcorner}$ou, para algum a $\in \Sigma$, $\gamma_{\mathrm{a} \rightarrow \mathbf{a}} \neq 0$, então pode ser o caso de que não seja possível representar por um alinhamento um conjunto de operações de edição de custo mínimo. Neste caso, definimos uma estrutura que 
tenha como finalidade essa representação e a chamamos de alinhamento estendido. Enquanto cada coluna de um alinhamento convencional representa uma única operação de edição, em um alinhamento estendido cada coluna representa uma sequência de operações de edição. Por exemplo,

$$
\left[\begin{array}{cccc} 
& & c & \\
a & & & \\
b & a & a \\
& & b & b
\end{array}\right]
$$

representa um alinhamento estendido de (aac, babb). Nesse exemplo, a primeira coluna representa que a foi substituído por b; a segunda coluna representa que a não sofreu alteração; a terceira coluna representa que c foi removido, depois um a foi inserido e depois substituído por b; e a quarta coluna representa que a foi inserido e depois substituído por b. Assim como no alinhamento convencional, a pontuação de um alinhamento estendido é a soma dos custos das operações de edição por ele representadas. $\mathrm{O}$ valor da função $o p t \mathrm{E}_{\gamma}$ que recebe como argumento um par ordenado de sequências $(s, t)$ é a pontuação do alinhamento estendido de $(s, t)$ de menor pontuação. Um algoritmo para esse critério é estudado nesta tese e, assim como para a função opt $\mathrm{A}_{\gamma}$, estudamos, para cada propriedade $P$ de uma métrica, as condições necessárias e suficientes da matriz de pontuação $\gamma$ para a função $o p t \mathrm{E}_{\gamma}$ possuir a propriedade $P$.

Voltando ao alinhamento convencional de duas sequências, um outro aspecto considerado na pontuação é o fato de que opt $\mathrm{A}_{\gamma}$ pode não descrever adequadamente o grau de semelhança entre duas sequências. Suponha, por exemplo, que duas sequências $s$ e $t$ diferem apenas em um símbolo. A diferença é representada por uma única operação de edição. Se, por exemplo, a quantidade de símbolos de cada uma das duas sequências é muito grande comparada com o custo da operação de edição envolvida, podemos dizer que as sequências são praticamente iguais. Por outro lado, se a quantidade de símbolos de cada uma das sequências é pequena, então as sequências podem ser consideradas muito diferentes. Levando em consideração a observação acima na pontuação de um alinhamento, Marzal e Vidal [MV93] definiram o critério $v \mathrm{~N}_{\gamma}$ que é a pontuação normalizada de um alinhamento (convencional). Mais precisamente, sendo $A$ um alinhamento de duas sequências, $v \mathrm{~N}_{\gamma}(A)=v \mathrm{~A}_{\gamma}(A) /|A|$, onde $|A|$ é o número de colunas de $A$. A função ótima para o critério $v \mathrm{~N}_{\gamma}$ é opt $\mathrm{N}_{\gamma}$, ou seja, opt $\mathrm{N}_{\gamma}$ é a pontuação- $v \mathrm{~N}_{\gamma}$ de um alinhamento de menor pontuação- $v \mathbf{N}_{\gamma}$ de duas sequências.

Diferentemente do critério $v \mathrm{~A}_{\gamma}$ para pontuar alinhamentos, $\gamma$ ser uma métrica em $\Sigma$ não implica que opt $\mathrm{N}_{\gamma}$ seja uma métrica em $\Sigma^{*}$. Essa observação foi feita por Marzal e Vidal [MV93] a partir da matriz de pontuação

$$
\gamma=\begin{array}{c|ccc} 
& \mathrm{a} & \mathrm{b} & \mathrm{c} \\
\hline \mathrm{a} & 0 & 5 & 5 \\
\mathrm{~b} & 5 & 0 & 1 \\
& 5 & 1 &
\end{array} .
$$

Note que $\gamma$ é uma métrica em $\Sigma_{\sqcup}$, mas opt $\mathrm{N}_{\gamma}$ não é métrica em $\Sigma^{*}$ desde que

$$
\begin{aligned}
\operatorname{opt} \mathrm{N}_{\gamma}(\mathrm{a}, \mathrm{b}) & =\min \left\{v \mathrm{~N}_{\gamma}\left(\left[\begin{array}{cc}
\mathrm{a} & \mathrm{b} \\
& \mathrm{b}
\end{array}\right]\right), v \mathrm{~N}_{\gamma}\left(\left[\begin{array}{cc}
\mathrm{b} & \mathrm{a} \\
\mathrm{b} & \sqcup
\end{array}\right]\right), v \mathrm{~N}_{\gamma}\left(\left[\begin{array}{l}
\mathrm{a} \\
\mathrm{b}
\end{array}\right]\right)\right\}=3 \\
& >\frac{17}{6}=\frac{1}{2}+\frac{7}{3}=v \mathrm{~N}_{\gamma}\left(\left[\begin{array}{cc}
\mathrm{a} & \sqcup \\
\mathrm{a} & \mathrm{b}
\end{array}\right]\right)+v \mathrm{~N}_{\gamma}\left(\left[\begin{array}{ccc}
\mathrm{a} & \mathrm{b} & \sqcup \\
\sqcup & \sqcup & \mathrm{b}
\end{array}\right]\right) \\
& \geq \operatorname{opt}_{\gamma}(\mathrm{a}, \mathrm{ab})+\operatorname{opt} \mathrm{N}_{\gamma}(\mathrm{ab}, \mathrm{b}) .
\end{aligned}
$$


Yujian e Bo [YB07] observaram que é um problema em aberto decidir se uma matriz $\gamma$ induz opt $\mathrm{N}_{\gamma}$ métrica em $\Sigma^{*}$. Em [AS06] resolvemos esse problema mostrando as condições suficientes e necessárias de $\gamma$ para opt $\mathrm{N}_{\gamma}$ ser uma métrica em $\Sigma^{*}$.

Quando usamos o critério $v \mathrm{~A}_{\gamma}$ e calculamos a pontuação de dois alinhamentos $A$ e $B$ de duas sequências e $v \mathrm{~A}_{\gamma}(A) \leq v \mathrm{~A}_{\gamma}(B)$, entendemos que, para o critério $v \mathrm{~A}_{\gamma}$, o alinhamento $A$ é "melhor" (ou pelo menos não é pior) do que o alinhamento $B$. Essa "classificação" depende da matriz de pontuação $\gamma$. Por exemplo, considere duas matrizes de pontuação $\gamma$ e $\delta$ tais que

$$
\gamma=\begin{array}{c|ccc} 
& \mathrm{a} & \mathrm{b} & \mathrm{c} \\
\hline \mathrm{a} & 0 & 1 & 1 \\
\mathrm{~b} & 1 & 0 & 1 \\
& 1 & 1
\end{array} \quad e \quad \delta=\begin{array}{r|ccc}
\mathrm{a} & 0 & 1 & 2 \\
\mathrm{~b} & 1 & 0 & 2 \\
& & 2 & 2
\end{array}
$$

e os seguintes alinhamentos de (aba, bab):

$$
A=\left[\begin{array}{lll}
\mathrm{a} & \mathrm{b} & \mathrm{a} \\
\mathrm{b} & \mathrm{a} & \mathrm{b}
\end{array}\right] \text { e } B=\left[\begin{array}{cccc}
\mathrm{a} & \mathrm{b} & \mathrm{a} & \mathrm{u} \\
& \mathrm{b} & \mathrm{a} & \mathrm{b}
\end{array}\right]
$$

Se utilizamos a matriz de pontuação $\gamma$, então $B$ é considerado como um alinhamento "melhor" do que $A$ pois

$$
v \mathrm{~A}_{\gamma}(B)=1+0+0+1=2<3=1+1+1=v \mathrm{~A}_{\gamma}(A),
$$

mas se utilizamos $\delta$, então é $A$ que é considerado "melhor" pois

$$
v \mathrm{~A}_{\delta}(A)=1+1+1=3<4=2+0+0+2=v \mathrm{~A}_{\delta}(B) .
$$

Entretanto, não é verdade que se $\gamma \neq \delta$, então existem sequências $s, t \in \Sigma^{*}$ e alinhamentos $A$ e $B$ de $(s, t)$ tais que $A$ é melhor que $B$ se a matriz de pontuação é $\gamma$ e $B$ é melhor que $A$ se a matriz de pontuação é $\delta$. Em outras palavras, pode acontecer de $\gamma \neq \delta$ e

$$
v \mathrm{~A}_{\gamma}(A) \leq v \mathrm{~A}_{\gamma}(B) \quad \text { se e somente se } \quad v \mathrm{~A}_{\delta}(A) \leq v \mathrm{~A}_{\delta}(B)
$$

para cada par de alinhamentos $A$ e $B$ de duas sequências. Se vale (1.2), dizemos que as matrizes de pontuação $\gamma$ e $\delta$ são equivalentes. Nesta tese, caracterizamos as matrizes de pontuação equivalentes para um conjunto amplo de matrizes de pontuação.

Um alinhamento de várias sequências é uma generalização do alinhamento de duas sequências. A pontuação-SP é um critério para pontuar um alinhamento de várias sequências que, assim como o critério $v \mathrm{~A}_{\gamma}$ para duas sequências, não considera o comprimento das sequências alinhadas mas somente a soma dos custos das operações de edição representadas nas colunas do alinhamento. Nesta tese, apresentamos três novos critérios para pontuar os alinhamentos e que representa, cada um deles, uma generalização do critério $v \mathrm{~N}_{\gamma}$ para alinhamento de várias sequências. Para cada um dos critérios propostos, mostramos um algoritmo exato para encontrar a pontuação de um alinhamento ótimo e mostramos que a versão de decisão do problema é NP-completa. Também mostramos um algoritmo para obter uma pontuação aproximada da pontuação ótima do alinhamento de várias sequências para a pontuação-SP quando a matriz de pontuação $\gamma$ induz métrica em opt $\mathrm{A}_{\gamma}$. Esse algoritmo pode ser utilizado para obter uma pontuação aproximada da pontuação ótima quando o critério utilizado é um dos três novos critérios definidos e a matriz de pontuação induz métrica em $o p t \mathrm{~N}_{\gamma}$. 


\subsection{Contribuições}

As principais contribuições desta tese e que foram discutidas na seção anterior são resumidas a seguir.

(a) Definimos alinhamento estendido de duas sequências, que é uma estrutura mais geral do que um alinhamento convencional e que utilizamos para representar um conjunto de operações de edição que transformam uma sequência em outra. Definimos também o critério opt $\mathrm{E}_{\gamma}$ para pontuar alinhamentos estendidos e descrevemos um algoritmo para computar opt $\mathrm{E}_{\gamma}$ e um alinhamento estendido ótimo.

(b) Descrevemos e ampliamos os resultados obtidos em [AS06] caracterizando, para cada propriedade $P$ de uma métrica, a classe das matrizes de pontuação $\gamma$ para as quais a função induzida opt $\mathrm{A}_{\gamma}$ possui a propriedade $P$ em $\Sigma^{*}$; e fazemos o mesmo considerando as funções opt $\mathrm{N}_{\gamma}$ e opt $\mathrm{E}_{\gamma}$.

(c) Mostramos como identificar matrizes de pontuação equivalentes para uma ampla classe de matrizes de pontuação.

(d) Definimos $\mathrm{V}_{\gamma}^{1}, \mathrm{~V}_{\gamma}^{2}$ e $\mathrm{V}_{\gamma}^{3}$, que são, como veremos, três novos critérios para a pontuação de um alinhamento de várias sequências. Eles levam em conta não somente a soma dos custos das operações de edição em um alinhamento mas também o seu comprimento. Para cada um desses critérios, descrevemos um algoritmo exato para encontrar a pontuação de um alinhamento ótimo e mostramos que a versão de decisão desse problema é NP-completa.

Descrevemos um algoritmo para encontrar uma pontuação aproximada da pontuação de um alinhamento ótimo de várias sequências para o critério pontuação-SP quando a matriz de pontuação $\gamma$ induz métrica na função opt $\mathrm{A}_{\gamma}$. Esse algoritmo pode ser utilizado também como algoritmo de aproximação para encontrar uma pontuação aproximada da pontuação de um alinhamento ótimo para o critério $\mathrm{V}_{\gamma}^{2}$ quando a matriz de pontuação $\gamma$ induz métrica em opt $\mathrm{N}_{\gamma}$.

\subsection{Organização do Trabalho}

Esta tese está organizada como descrito a seguir. No Capítulo 2 apresentamos os conceitos e os principais resultados da revisão bibliográfica que julgamos mais importantes para este trabalho. No Capítulo 3 apresentamos o conceito de alinhamento estendido, o critério $v \mathrm{E}_{\gamma}$ para pontuar um alinhamento estendido e um algoritmo para encontrar a pontuação de um alinhamento estendido ótimo, assim como o alinhamento estendido correspondente. No Capítulo 4 caracterizamos as matrizes de pontuação $\gamma$ que induzem funções opt $\mathrm{A}_{\gamma}$ que são métricas nas sequências; e fazemos o mesmo com as funções opt $\mathrm{N}_{\gamma}$ e opt $\mathrm{E}_{\gamma}$. No Capítulo 5 mostramos como determinar se duas matrizes de pontuação $\gamma$ e $\delta$ são equivalentes. No Capítulo 6 descrevemos os resultados para alinhamentos de várias sequências. Finalmente, no Capítulo 7 apresentamos as conclusões obtidas neste trabalho, sugerindo trabalhos futuros. 


\section{Capítulo 2}

\section{Conceitos}

Neste capítulo dissertamos sobre os conceitos utilizados nesta tese. Descrevemos os principais algoritmos e a complexidade dos problemas estudados e que são mencionados na literatura. Na Seção 2.1 fazemos as definições básicas que são utilizadas nas demais seções e nos capítulos seguintes. Nas Seções 2.2 e 2.3 apresentamos alguns resultados encontrados em nossa pesquisa bibliográfica sobre alinhamentos de duas e de várias sequências.

\subsection{Preliminares}

\subsubsection{Alfabeto e sequências}

Um alfabeto $\Sigma$ é um conjunto finito e não-vazio de símbolos. Denotamos uma sequência finita $s$ sobre $\Sigma$ por $s(1) s(2) \ldots s(n)$, onde $s(j) \in \Sigma$. Dizemos que o comprimento de $s$, denotado por $|s|$, é $n$. Denotamos a sequência $s(p) s(p+1) \ldots s(q)$ por $s(p \ldots q)$, e a sequência vazia, que é a sequência de comprimento igual a zero, por $\epsilon$. A sequência a ${ }^{n}$ é a sequência formada por $n$ símbolos a. Denotamos a sequência formada pela concatenação das sequências $s$ e $t$ por $s t$.

\subsubsection{Alinhamentos}

Seja $\Sigma_{\sqcup}=\Sigma \cup\left\{\_\right\}$, onde $\sqcup \notin \Sigma$. Chamamos espaço o símbolo ${ }_{\sqcup}$. Ele é usado para representar uma inserção ou uma remoção em operações de edição. Seja $S=\left(s_{1}, \ldots, s_{k}\right)$ uma $k$-upla de elementos em $\Sigma^{*}$. Um alinhamento de $S$ é uma $k$-upla $A=\left(s_{1}^{\prime}, \ldots, s_{k}^{\prime}\right)$ de elementos em $\Sigma^{*}$, onde

(a) cada sequência $s_{h}^{\prime}$ é obtida inserindo-se espaços em $s_{h}$,

(b) $\left|s_{h}^{\prime}\right|=\left|s_{i}^{\prime}\right|$ para cada $h, i$ e

(c) não existe $j$ tal que $s_{1}^{\prime}(j)=\ldots=s_{k}^{\prime}(j)=$.

Quando o alfabeto dos símbolos que formam os elementos de uma $k$-upla é $\Sigma$, preferimos escrevê-la entre colchetes "[" e "]". Por outro lado, usamos parênteses "(" e ")" para escrever $k$-uplas cujos elementos são formados por símbolos em $\Sigma$. Então, preferimos escrever $\left[s_{1}^{\prime}, \ldots, s_{k}^{\prime}\right]$ em vez de $\left(s_{1}^{\prime}, \ldots, s_{k}^{\prime}\right)$ para denotar o alinhamento formado pelas sequências $s_{1}^{\prime}, \ldots, s_{k}^{\prime}$. Dizemos que a $k$-upla de símbolos $\left[s_{1}^{\prime}(j), \ldots, s_{k}^{\prime}(j)\right]$ é a coluna $j$ do alinhamento $\left[s_{1}^{\prime}, \ldots, s_{k}^{\prime}\right]$ e denotamos a coluna $j$ de um alinhamento $A$ por $A(j)$. Se

$$
A=\left[\ldots, s_{h}^{\prime}, \ldots, s_{i}^{\prime}, \ldots\right]
$$


dizemos que o par de símbolos $\left[s_{h}^{\prime}(j), s_{i}^{\prime}(j)\right]$ alinha em $A$ ou, simplesmente, que $s_{h}^{\prime}(j)$ e $s_{i}^{\prime}(j)$ estão alinhados em $A$ se a ordem está subentendida, e que $|A|=\left|s_{i}^{\prime}\right|$ é o comprimento do alinhamento $A$.

São conhecidos os seguintes limitantes para o comprimento de um alinhamento.

Fato 1. Seja $A$ um alinhamento de $\left(s_{1}, \ldots, s_{k}\right)$. Então, $\max _{i}\left\{\left|s_{i}\right|\right\} \leq|A| \leq \sum_{i}\left|s_{i}\right|$.

Prova. Suponha que $i$ é um inteiro e $1 \leq i \leq k$. Observe que os símbolos de $s_{i}$ devem estar em colunas distintas em $A$. Logo, $A$ deve ter pelo menos $\left|s_{i}\right|$ colunas. Como a observação vale para qualquer $i$, temos que $\max _{i}\left\{\left|s_{i}\right|\right\} \leq|A|$.

Pela definição de alinhamento, cada coluna de $A$ deve ter pelo menos um símbolo de alguma sequência em $s_{1}, \ldots, s_{k}$. Como a quantidade de símbolos em $\left(s_{1}, \ldots, s_{k}\right)$ é $\sum_{i}\left|s_{i}\right|$, segue que $|A| \leq \sum_{i}\left|s_{i}\right|$.

Segue dos resultados acima que $\max _{i}\left\{\left|s_{i}\right|\right\} \leq|A| \leq \sum_{i}\left|s_{i}\right|$.

Um alinhamento também pode ser representado no formato matricial colocando uma sequência sobre outra. Assim, os alinhamentos

$$
\left[\mathrm{aaa}_{\sqcup}, \mathrm{ab}_{\sqcup \sqcup,}, \mathrm{cac}\right] \text { e }\left[\mathrm{aaa}_{\sqcup}, \mathrm{ab}_{\sqcup \sqcup \sqcup,}, \mathrm{ca}_{\sqcup} \mathrm{c}\right]
$$

de (aaa, ab, cac) podem ser visualizados por

$$
\left[\begin{array}{cccc}
a & a & a & - \\
a & b & \sqcup & \sqcup \\
& c & a & c
\end{array}\right] \text { e }\left[\begin{array}{ccccc}
\sqcup & a & a & a & \sqcup \\
a & b & \sqcup & u & \sqcup \\
\sqcup & c & a & u & c
\end{array}\right] \text {. }
$$

Sejam $I=\left\{i_{1}, \ldots, i_{m}\right\} \subseteq\{1, \ldots, k\}$ tal que $i_{1}<\ldots<i_{m}$ e $A=\left[s_{1}^{\prime}, \ldots, s_{k}^{\prime}\right]$ um alinhamento de $S=\left(s_{1}, \ldots, s_{k}\right)$. Escrevemos $S_{I}$ para denotar a $m$-upla $\left(s_{i_{1}}, \ldots, s_{i_{m}}\right)$. O alinhamento de $S_{I}$ induzido por $A$ é o alinhamento $A_{I}$ obtido a partir do alinhamento $A$ considerando somente as sequências correspondentes às sequências em $S_{I}$ e, da estrutura resultante, eliminando as colunas onde todos os símbolos são iguais a ${ }_{\omega}$.

No exemplo acima,

$$
\left[\begin{array}{lll}
\mathrm{a} & \mathrm{a} & \mathrm{a} \\
\mathrm{a} & \mathrm{b} & \mathrm{c}
\end{array}\right]
$$

é um alinhamento de (aaa, ab) induzido por [aaa $, a_{\sqcup \sqcup}, b_{\sqcup} c a c$ ] de (aaa, ab, cac).

Denotamos por $\mathcal{A}_{S}$ o conjunto de todos os alinhamentos de $S$.

\subsubsection{Matrizes de pontuação}

Cada critério de pontuação aqui estudado tem uma matriz de pontuação associada à sua definição. Uma matriz de pontuação é uma matriz que possui números reais como entradas e que são indexadas nas linhas e nas colunas por elementos em $\Sigma_{\sqcup}$. Para a, b $\in \Sigma_{\sqcup}$ e uma matriz de pontuação $\gamma$, denotamos por $\gamma_{\mathrm{a} \rightarrow \mathrm{b}}$ a entrada de $\gamma$ na linha a e coluna $\mathrm{b}$. O valor de $\gamma_{a \rightarrow b}$ define um custo para a operação de edição de substituição se $a, b \in \Sigma$, de inserção

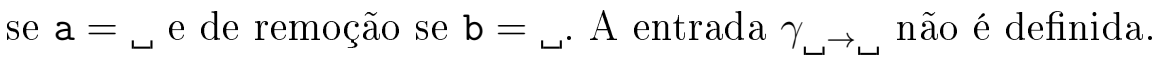




\subsubsection{Digrafos}

Um digrafo $D$ consiste de um conjunto finito $V(D)$ de elementos chamados vértices, um conjunto $E(D)$ de pares ordenados de vértices chamados arcos e uma função

$$
\text { custo }: E(D) \rightarrow \mathbb{R}
$$

$\mathrm{O} \operatorname{arco}(v, w) \in E(D)$ é denotado por $v \rightarrow w$ e dizemos que $v \rightarrow w$ sai do vértice $v$ e que entra no vértice $w$. Dizemos que o custo do arco e é custo(e) e, sobrecarregando a função custo, definimos o custo do digrafo $D$ como custo $(D)=\sum_{e \in E(D)}$ custo $(e)$.

Sejam $v_{0}, v_{1}, \ldots, v_{n} \in V(D)$ e $e_{1}, e_{2}, \ldots, e_{n} \in E(D)$, tais que $e_{i}=v_{i-1} \rightarrow v_{i}$ para cada $i$. Dizemos que a sequência de $\operatorname{arcos} P=\left(e_{1}, \ldots, e_{n}\right)$ é um caminho de comprimento $n$ de $v_{0}$ a $v_{n}$, que cada arco $e_{i}$ aparece em $P$, e que $v_{0}, v_{1}, \ldots, v_{n}$ e $e_{1}, e_{2}, \ldots, e_{n}$ são, respectivamente, os vértices e os arcos do caminho $P$. O custo do caminho $P$ é custo $(P)=\sum_{i=1}^{n}$ custo $\left(e_{i}\right)$. Se não existe um caminho $P^{\prime}$ de $v_{0}$ a $v_{n}$ tal que custo $\left(P^{\prime}\right)<\operatorname{custo}(P)$, dizemos que $P$ é um caminho ótimo de $v_{0}$ a $v_{n}$. Se $v_{0}=v_{n}$, dizemos que $P$ é um ciclo. Se $P$ é um ciclo e custo $(P)<0$, dizemos que $P$ é um ciclo negativo. Se os arcos em um ciclo $P$ são dois a dois distintos, dizemos que $P$ é um ciclo simples. Dizemos que um conjunto de $\operatorname{arcos}\left\{e_{1}, \ldots, e_{n}\right\}$ define o ciclo $P$ se $P$ é um ciclo de comprimento $n$, é simples e cada $e_{i}$ aparece em $P$.

Sejam $P=\left(e_{1}, \ldots, e_{n}\right)$ e $Q=\left(f_{1}, \ldots, f_{m}\right)$ caminhos de $v$ a $z$ e de $z$ a $w$ respectivamente. Então, denotamos a concatenação dos caminhos $P$ e $Q$ por

$$
P Q=\left(e_{1}, e_{2}, \ldots, e_{n}, f_{1}, f_{2}, \ldots, f_{m}\right)
$$

que é um caminho de $v$ a $w$.

Dizemos que um digrafo $D$ é euleriano se, para cada vértice $v \in V(D)$, as quantidades de arcos que entram e que saem de $v$ são iguais. Um resultado bem conhecido $\left[\mathrm{GPB}^{+} 02\right]$ é o seguinte.

Teorema 2. Um digrafo $D$ é euleriano se e somente se existe uma partição de $E(D)$ onde cada conjunto da partição define um ciclo.

Para uma matriz de pontuação $\gamma$, o digrafo $D(\gamma)$ é tal que

$$
\begin{aligned}
V(D) & =\Sigma_{\sqcup}, \\
E(D) & =\left(\Sigma_{\sqcup} \times \Sigma_{\sqcup}\right) \backslash\left\{\left({ }_{\sqcup}, \sqcup\right)\right\} \mathrm{e} \\
\text { custo }(v \rightarrow w) & =\gamma_{v \rightarrow w} \text { para cada } v \rightarrow w \in E(D) .
\end{aligned}
$$

\subsubsection{Problemas}

Uma instância de um problema clássico de alinhamento em geral é um conjunto de sequências. Sendo um conjunto, a ordem das sequências na instância não é importante. Implicitamente nesses casos a matriz de pontuação deve ser simétrica. Em outras palavras, esse é um caso particular quando assumimos que uma instância é uma lista ordenada de sequências e que as matrizes utilizadas podem não ser simétricas. Consideramos em nossas definições a formulação mais geral.

Os problemas envolvendo alinhamento de sequências têm como objetivo encontrar um alinhamento ótimo. Em geral, o tempo gasto para encontrar um alinhamento ótimo e o tempo gasto para encontrar a pontuação de um alinhamento ótimo são assintoticamente iguais. Para o critério $v \mathrm{~A}_{\gamma}$, Needleman e Wunsch [NW70] descrevem um algoritmo que, a partir de uma tabela de programação dinâmica, encontra um alinhamento ótimo de duas 
sequências $s$ e $t$. Para outros critérios estudados neste texto, inclusive aqueles utilizados para várias sequências, uma adaptação óbvia desse método pode ser usada para encontrar um alinhamento de pontuação ótima consultando uma tabela de programação dinâmica. Então, desde que os algoritmos apresentados em geral constroem tabelas de programação dinâmica, optamos neste texto em definir e tratar os problemas de alinhamento como um problema para encontrar somente a pontuação de um alinhamento ótimo de uma $k$-upla de sequências, omitindo detalhes de como um alinhamento ótimo pode ser obtido.

Além de problemas de otimização, também tratamos de problemas de decisão para discutir resultados de complexidade. Neste caso, denotamos por $\mathbf{P}(I)$ a resposta ou solução de um problema de decisão $\mathbf{P}$ para a instância $I$, ou seja, $\mathbf{P}(I) \in\{\operatorname{Sim}, \mathbf{N a ̃ o}\}$.

\subsubsection{Fator de aproximação}

Sejam II o conjunto de instâncias de um problema de minimização, OPT $(I)$ um número associado a cada instância $I \in \mathbb{I}$ e $\mathscr{A}(I) \geq \mathrm{OPT}(I)$ um número computado por um algoritmo $\mathscr{A}$ com entrada $I$. Dizemos que $\mathscr{A}$ é uma $\alpha$-aproximação para o problema se

$$
\mathscr{A}(I) \leq \alpha \cdot \operatorname{OPT}(I)
$$

para toda instância $I$, onde $\alpha$ é um número constante. Dizemos também que $\alpha$ é uma fator de aproximação do algoritmo $\mathscr{A}$.

\subsection{Alinhamento de duas sequências}

\subsubsection{Critério $v \mathrm{~A}_{\gamma}$}

Sejam $s, t \in \Sigma^{*}, n=|s|, m=|t|$. Um critério simples para pontuar um alinhamento usa a função $v \mathrm{~A}_{\gamma}$. Para um alinhamento $\left[s^{\prime}, t^{\prime}\right]$ de $(s, t)$ temos que

$$
v \mathrm{~A}_{\gamma}\left(\left[s^{\prime}, t^{\prime}\right]\right)=\sum_{j=1}^{\left|\left[s^{\prime}, t^{\prime}\right]\right|} \gamma_{s^{\prime}(j) \rightarrow t^{\prime}(j)} .
$$

Dizemos que $v \mathrm{~A}_{\gamma}\left(\left[s^{\prime}, t^{\prime}\right]\right)$ é a pontuação-v $\mathrm{A}_{\gamma}$ do alinhamento $\left[s^{\prime}, t^{\prime}\right]$.

A função ótima para esse critério é opt $\mathrm{A}_{\gamma}$, ou seja, opt $\mathrm{A}_{\gamma}(s, t)=\min _{A \in \mathcal{A}_{\{s, t\}}}\left\{v \mathrm{~A}_{\gamma}(A)\right\}$. Um alinhamento $A$ de $(s, t)$ tal que $v \mathrm{~A}_{\gamma}(A)=o p t \mathrm{~A}_{\gamma}(s, t)$ é chamado de alinhamento $A$-ótimo de $(s, t)$. O problema correspondente é

Problema 1. APS: Alinhamento de um par de sequências

Dados $s, t \in \Sigma^{*}$, determinar opt $\mathrm{A}_{\gamma}(s, t)$, onde $\gamma$ é uma matriz de pontuação fixa.

Um algoritmo ingênuo para a solução do Problema APS calcula as pontuações- $v \mathrm{~A}_{\gamma}$ de todos os alinhamentos em $\mathcal{A}_{\{s, t\}}$ e devolve a menor pontuação computada. O problema com essa estratégia é que, em geral, o valor de $\left|\mathcal{A}_{\{s, t\}}\right|$ é muito grande. Estimamos a seguir esse valor supondo que $n=|s|=|t|$.

Primeiramente contamos quantos alinhamentos diferentes $\left[s^{\prime}, t^{\prime}\right]$ de comprimento $2 n-j$ existem para um dado inteiro $j$. Como $\left|\left[s^{\prime}, t^{\prime}\right]\right|=2 n-j$, temos que $\left|s^{\prime}\right|=\left|t^{\prime}\right|=2 n-j$, o que implica, sendo $n=|s|=|t|$, que as sequências $s^{\prime}$ e $t^{\prime}$ possuem, cada uma, $n-j$ espaços. Sendo $\left|s^{\prime}\right|=2 n-j$, segue que existem $\left(\begin{array}{c}2 n-j \\ n-j\end{array}\right)$ modos diferentes de definir a posição dos $n-j$ espaços em $s^{\prime}$. Uma vez feito isto, desde que $\left|t^{\prime}\right|=2 n-j$ e que nenhuma coluna de $\left[s^{\prime}, t^{\prime}\right]$ pode possuir dois caracteres iguais a espaço, há $\left(\begin{array}{c}(2 n-j)-(n-j) \\ n-j\end{array}\right)=\left(\begin{array}{c}n \\ n-j\end{array}\right)$ modos diferentes de 
definir a posição dos $n-j$ espaços na sequência $t^{\prime}$. Logo, a quantidade de alinhamentos $\left[s^{\prime}, t^{\prime}\right]$ distintos de comprimento $2 n-j$ é

$$
\left(\begin{array}{c}
2 n-j \\
n-j
\end{array}\right) \cdot\left(\begin{array}{c}
n \\
n-j
\end{array}\right)=\frac{(2 n-j) !}{(n-j) ! n !} \cdot \frac{n !}{(n-j) ! j !}=\frac{(2 n-j) !}{(n-j) !(n-j) ! j !} .
$$

Portanto, considerando $j=0,1, \ldots, n$, segue

$$
\left|\mathcal{A}_{\{s, t\}}\right|=\sum_{j=0}^{n} \frac{(2 n-j) !}{(n-j) !(n-j) ! j !} .
$$

O seguinte fato estabelece um limite inferior para o valor de (2.1).

Fato 3. Sejam $s, t \in \Sigma \operatorname{com} n=|s|=|t|$. Então, $\left|\mathcal{A}_{\{s, t\}}\right|=\Omega\left(4^{n}\right)$.

Prova. Para mostrar que $\left|\mathcal{A}_{\{s, t\}}\right|=\Omega\left(4^{n}\right)$, basta mostrar que para $n \geq 1$, temos que

$$
\frac{(2 n-1) !}{(n-1) !(n-1) !} \geq\left(\frac{1}{4}\right) 4^{n}
$$

desde que $(2 n-1) ! /((n-1) !(n-1)$ !) é apenas uma das parcelas da expressão em $(2.1)$.

A prova é por indução em $n$. Note primeiramente que, para $n=1$, temos

$$
\frac{(2-1) !}{(1-1) !(1-1) !}=1=\left(\frac{1}{4}\right) 4^{1}
$$

Suponha agora que $n>1$ e que

$$
\frac{(2(n-1)-1) !}{((n-1)-1) !((n-1)-1) !} \geq\left(\frac{1}{4}\right) 4^{n-1} .
$$

Logo,

$$
\begin{aligned}
\frac{(2 n-1) !}{(n-1) !(n-1) !} & =\frac{(2 n-1)(2 n-2)}{(n-1)(n-1)} \frac{(2(n-1)-1) !}{((n-1)-1) !((n-1)-1) !} \\
& \geq \frac{(2 n-1)(2 n-2)}{(n-1)(n-1)}\left(\frac{1}{4}\right) 4^{n-1} \\
& \geq \frac{(2 n-2)(2 n-2)}{(n-1)(n-1)}\left(\frac{1}{4}\right) 4^{n-1} \\
& =\left(\frac{1}{4}\right) 4^{n},
\end{aligned}
$$

onde (2.2) segue da hipótese de indução e de $n>1$, e (2.3) segue de $n>1$.

Então, o problema de usar um algoritmo que use uma estratégia para buscar um alinhamento A-ótimo verificando todos os possíveis alinhamentos, pelo Fato 3, gasta tempo exponencial no tamanho da entrada no caso geral, o que torna a execução desse algoritmo impraticável.

Needleman e Wunsch [NW70] descrevem um algoritmo de programação dinâmica para o Problema APS. Para mostrar o funcionamento do algoritmo, considere as sequências $s, t \in \Sigma$ com $n, m$ símbolos cada, respectivamente, e $A=\left[s^{\prime}(1) \ldots s^{\prime}(\mathcal{N}), t^{\prime}(1) \ldots t^{\prime}(\mathcal{N})\right]$ um alinhamento A-ótimo de $(s, t)$, ou seja, opt $\mathrm{A}_{\gamma}(s, t)=v \mathrm{~A}_{\gamma}(A)$. Para o cálculo de $v \mathrm{~A}_{\gamma}(A)$, dois 
casos devem ser considerados: ou uma das sequências $s$ ou $t$ é vazia, ou as duas sequências são não vazias. Se $s=\epsilon$ ou $t=\epsilon$, então somente um alinhamento é possível e

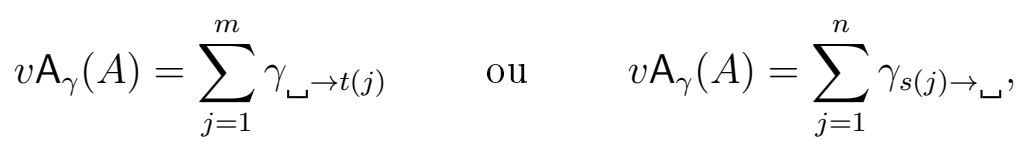

respectivamente. Se as duas sequências são não vazias, então primeiramente note que se $A^{\prime}=\left[s^{\prime}(1) \ldots s^{\prime}(\mathcal{N}-1), t^{\prime}(1) \ldots t^{\prime}(\mathcal{N}-1)\right]$, então

$$
v \mathrm{~A}_{\gamma}(A)=v \mathrm{~A}_{\gamma}\left(A^{\prime}\right)+\gamma_{s^{\prime}(\mathcal{N}) \rightarrow t^{\prime}(\mathcal{N})} .
$$

Além disso, existem $\bar{s}, \bar{t} \in \Sigma^{*}$ tais que $A^{\prime}$ é um alinhamento de $(\bar{s}, \bar{t})$. Vamos mostrar que $A^{\prime}$ é um alinhamento A-ótimo de $(\bar{s}, \bar{t})$. Suponha, por contradição, que $A^{\prime}$ não é A-ótimo. Então, existe um alinhamento $\left[\bar{s}^{\prime}, \bar{t}^{\prime}\right]$ de $(\bar{s}, \bar{t})$ tal que $v \mathrm{~A}_{\gamma}\left(\left[\bar{s}^{\prime}, \bar{t}^{\prime}\right]\right)<v \mathrm{~A}_{\gamma}\left(A^{\prime}\right)$. Desde que $\left[\bar{s}^{\prime}(1) \ldots \bar{s}^{\prime}\left(\left|\left[\bar{s}^{\prime}, \bar{t}^{\prime}\right]\right|\right) s^{\prime}(\mathcal{N}), \bar{t}^{\prime}(1) \ldots \bar{t}^{\prime}\left(\left|\left[\bar{s}^{\prime}, \bar{t}^{\prime}\right]\right|\right) t^{\prime}(\mathcal{N})\right]$ também é um alinhamento de $(s, t)$, segue das observações acima que

$$
\begin{aligned}
v \mathrm{~A}_{\gamma}\left(\left[\bar{s}^{\prime}(1) \ldots \bar{s}^{\prime}\left(\left|\left[\bar{s}^{\prime}, \bar{t}^{\prime}\right]\right|\right) s^{\prime}(\mathcal{N}), \bar{t}^{\prime}(1) \ldots \bar{t}^{\prime}\left(\left|\left[\overline{s^{\prime}}, \bar{t}^{\prime}\right]\right|\right) t^{\prime}(\mathcal{N})\right]\right) & =v \mathrm{~A}_{\gamma}\left(\left[\overline{s^{\prime}}, \bar{t}^{\prime}\right]\right)+\gamma_{s^{\prime}(\mathcal{N}) \rightarrow t^{\prime}(\mathcal{N})} \\
& <v \mathrm{~A}_{\gamma}\left(A^{\prime}\right)+\gamma_{s^{\prime}(\mathcal{N}) \rightarrow t^{\prime}(\mathcal{N})} \\
& =v \mathrm{~A}_{\gamma}(A)=\operatorname{opt}_{\gamma}(s, t)
\end{aligned}
$$

contrariando a otimalidade de $A$. Portanto, $A^{\prime}$ é um alinhamento A-ótimo de $(\bar{s}, \bar{t})$.

Estudamos as três únicas possibilidades para $s^{\prime}(\mathcal{N})$ e $t^{\prime}(\mathcal{N})$ :

1. se $s^{\prime}(\mathcal{N}) \neq \neq_{\sqcup}$ e $t^{\prime}(\mathcal{N})={ }_{\iota}$, então $A^{\prime}$ é alinhamento ótimo de $(s(1 \ldots n-1), t)$, o que implica que

$$
o p t \mathrm{~A}_{\gamma}(s, t)=v \mathrm{~A}_{\gamma}\left(A^{\prime}\right)+\gamma_{s^{\prime}(\mathcal{N}) \rightarrow t^{\prime}(\mathcal{N})}=\operatorname{opt}_{\gamma}(s(1 \ldots n-1), t)+\gamma_{s(n) \rightarrow \sqcup ;} ;
$$

2. se $s^{\prime}(\mathcal{N}) \neq{ }_{\sqcup}$ e $t^{\prime}(\mathcal{N}) \neq \_$, então $A^{\prime}$ é alinhamento ótimo de $(s(1 \ldots n-1), t(1 \ldots m-1))$, o que implica que

$$
\operatorname{opt} \mathrm{A}_{\gamma}(s, t)=v \mathrm{~A}_{\gamma}\left(A^{\prime}\right)+\gamma_{s^{\prime}(\mathcal{N}) \rightarrow t^{\prime}(\mathcal{N})}=\operatorname{optA}_{\gamma}(s(1 \ldots n-1), t(1 \ldots m-1))+\gamma_{s(n) \rightarrow t(m)} \text {; }
$$

3. se $s^{\prime}(\mathcal{N})={ }$ e $t^{\prime}(\mathcal{N}) \neq{ }$, então $A^{\prime}$ é alinhamento ótimo de $(s, t(1 \ldots m-1))$, o que implica que

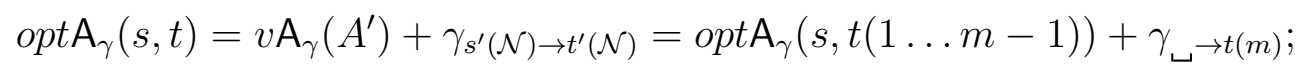

e escolhemos aquela com menor pontuação- $v \mathrm{~A}_{\gamma}$. Dessas considerações segue que

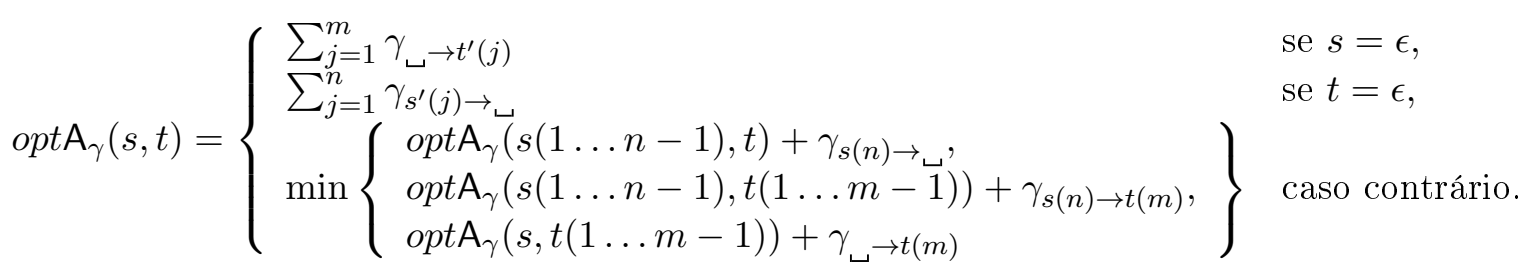

A recorrência é calculada usando uma tabela $d$ de programação dinâmica. $O$ valor de $d(p, q)$ é o valor de opt $\mathrm{A}_{\gamma}(s(1 \ldots p), t(1 \ldots q))$. A tabela $d$ é usualmente denominada matriz 
dos alinhamentos de $(s, t)$ e é construída conforme a função de recorrência

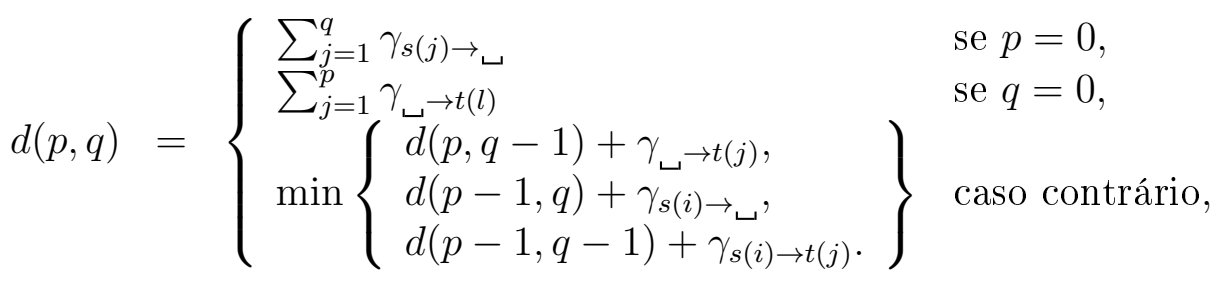

gastando tempo e espaço $\Theta\left(n^{2}\right)$ quando $n=m$. Claramente, $d(n, m)=\operatorname{opt}_{\gamma}(s, t)$.

Para o caso especial da matriz de pontuação $\gamma$ ser tal que $\gamma_{a \rightarrow b}=0$ se $a=b$ e $\gamma_{a \rightarrow b}=1$ se $\mathrm{a} \neq \mathrm{b}$, Masek e Paterson [MP80] descrevem um algoritmo que usa o método conhecido como "Método dos Quatro Russos" e que gasta tempo $O\left(n^{2} / \log n\right)$ quando $|s|=|t|=n$. Crochemore, Landau e Ziv-Ukelson [CLZU02] estenderam o método para matrizes com entradas racionais descrevendo assim um algoritmo para o problema que gasta tempo $O\left(n^{2} / \log n\right)$.

No restante deste capítulo, assumimos que o tempo gasto para determinar opt $\mathrm{A}_{\gamma}(s, t)$ e um alinhamento A-ótimo, onde $|s|=|t|=n$, no pior caso é $T_{\mathrm{APS}}(n)$.

\subsubsection{Critério $v \mathrm{~N}_{\gamma}$}

Marzal e Vidal [MV93] definem um outro critério para pontuar alinhamentos de duas sequências que chamamos pontuação-v $\mathrm{N}_{\gamma}$ que é uma normalização do valor da pontuação$v \mathrm{~A}_{\gamma}$. Eles definem a pontuação de um alinhamento $A$ de duas sequências como

$$
v \mathrm{~N}_{\gamma}(A)= \begin{cases}0 & \text { se }|A|=0 \\ v \mathrm{~A}_{\gamma}(A) /|A| & \text { caso contrário. }\end{cases}
$$

A função ótima para esse critério é

$$
\operatorname{opt} \mathrm{N}_{\gamma}(s, t)=\min _{A \in \mathcal{A}_{s, t}}\left\{v \mathrm{~N}_{\gamma}(A)\right\}
$$

e um alinhamento $N$-ótimo de $(s, t)$ é um alinhamento $A$ tal que $v \mathrm{~N}_{\gamma}(A)=o p t \mathrm{~N}_{\gamma}(s, t)$ e o problema correspondente é o seguinte.

Problema 2. APSN: Alinhamento de um par de sequências com custo normalizado

Dados $s, t \in \Sigma^{*}$, determinar opt $\mathrm{N}_{\gamma}(s, t)$, onde $\gamma$ é uma matriz de pontuação fixa.

Para a descrição dos algoritmos nesta seção assumimos que $n \neq 0$ ou $m \neq 0$.

Para uma matriz de pontuação $\gamma$ denotamos por $\gamma_{\min }$ e $\gamma_{\max }$ o menor e o maior valor de entrada da matriz $\gamma$. A seguir mostramos limitantes inferior e superior para o valor da pontuação- $v \mathrm{~N}_{\gamma}$ que dependem unicamente de $\gamma$.

Fato 4. Seja $A$ um alinhamento de $(s, t) \operatorname{com} s \neq \epsilon$ ou $t \neq \epsilon$. Então, $\gamma_{\min } \leq v \mathrm{~N}_{\gamma}(A) \leq \gamma_{\max }$.

Prova. Suponha que $\left[s^{\prime}, t^{\prime}\right]=A$. Como $s \neq \epsilon$ ou $t \neq \epsilon$, temos que $\left|\left[s^{\prime}, t^{\prime}\right]\right| \geq 1$. Logo,

$$
\begin{aligned}
\gamma_{\min }=\frac{\left|\left[s^{\prime}, t^{\prime}\right]\right| \gamma_{\min }}{\left|\left[s^{\prime}, t^{\prime}\right]\right|} \leq \frac{\sum_{i} \gamma_{s^{\prime}(i) \rightarrow t^{\prime}(i)}}{\left|\left[s^{\prime}, t^{\prime}\right]\right|} & =v \mathrm{~N}_{\gamma}\left(\left[s^{\prime}, t^{\prime}\right]\right) \\
& =\frac{\sum_{i} \gamma_{s^{\prime}(i) \rightarrow t^{\prime}(i)}}{\left|\left[s^{\prime}, t^{\prime}\right]\right|} \leq \frac{\left|\left[s^{\prime}, t^{\prime}\right]\right| \gamma_{\max }}{\left|\left[s^{\prime}, t^{\prime}\right]\right|}=\gamma_{\max } .
\end{aligned}
$$




\section{Uma heurística para calcular o valor de $\operatorname{opt} \mathrm{N}_{\gamma}(s, t)$}

Estendendo a idéia de Needleman e Wunsch, descrevemos uma heurística ingênua para o Problema 2. A estratégia é computar opt $\mathrm{A}_{\gamma}(s, t) /|A|$, onde $A$ é um alinhamento A-ótimo que tenha o maior comprimento. A idéia é adaptar o algoritmo que computa a tabela $d$ e que é descrito na Seção 2.2.1 para, quando calcular o valor $d(i, j)=\operatorname{optA}_{\gamma}(s(1 \ldots i), t(1 \ldots j))$, calcular também o valor de $L(i, j)$ que definimos ser o comprimento do alinhamento A-ótimo de maior comprimento de $(s(1 \ldots i), t(1 \ldots j))$.

Similarmente à computação de $d(i, j)$, o valor $L(i, j)$ é calculado usando programação dinâmica. A seguinte recorrência faz o trabalho. Para $i, j>0$,

$$
\begin{aligned}
& L(0,0)=0, \\
& L(0, j)=j \text {, } \\
& L(i, 0)=i \text {, } \\
& L(i, j)=\max \left\{\begin{array}{ll}
L(i-1, j) & \text { se } d(i, j)=d(i-1, j)+\gamma_{s(i) \rightarrow \sqcup,} \\
L(i, j-1) & \text { se } d(i, j)=d(i, j-1)+\gamma_{\sqcup \rightarrow t(j)}, \\
L(i-1, j-1) & \text { se } d(i, j)=d(i-1, j-1)+\gamma_{s(i) \rightarrow s(j)}
\end{array}\right\}+1 .
\end{aligned}
$$

O valor devolvido então pela heurística é $d(n, m) / L(n, m)$. Claramente a recorrência acima pode ser calculada usando tempo e espaço $\Theta\left(n^{2}\right)$ se $n=m$.

A heurística acima devolve o valor normalizado de um alinhamento A-ótimo de maior comprimento. Entretanto, dependendo das sequências envolvidas, nem sempre existe um alinhamento que é ao mesmo tempo A-ótimo e N-ótimo. Por exemplo, para

$$
\gamma_{a \rightarrow b}= \begin{cases}0 & \text { se } a=b, \\ 1 & \text { caso contrário, }\end{cases}
$$

os alinhamentos A-ótimo de maior comprimento e N-ótimos para (aab, bcc) são, respectivamente,

$$
\left[\begin{array}{lll}
a & a & b \\
b & c & c
\end{array}\right] \text { e }\left[\begin{array}{ccccc}
a & a & b & c & \sqcup \\
& \sqcup & b & c & c
\end{array}\right] \text {. }
$$

A heurística acima não garante encontrar a pontuação- $v \mathrm{~N}_{\gamma}$ de um alinhamento N-ótimo mas o valor computado é, no máximo, o dobro de opt $\mathrm{N}_{\gamma}(s, t)$, ou seja, a heurística é um algoritmo que é uma 2-aproximação. Para verificar esse fato, considere $A$ um alinhamento A-ótimo (cuja pontuação- $v \mathrm{~A}_{\gamma}$ foi calculada pelo algoritmo) e $B$ um alinhamento N-ótimo. Logo, $v \mathrm{~A}_{\gamma}(A) \leq v \mathrm{~A}_{\gamma}(B)$. Além disso,

$$
|B| \leq|s|+|t| \leq 2 \max \{|s|,|t|\} \leq 2|A|,
$$

onde a primeira e a última desigualdade seguem do Fato 1. Portanto, $|A| \geq(1 / 2)|B|$. Seguem das observações acima que

$$
v \mathrm{~N}_{\gamma}(A)=\frac{v \mathrm{~A}_{\gamma}(A)}{|A|} \leq \frac{v \mathrm{~A}_{\gamma}(B)}{|A|} \leq \frac{v \mathrm{~A}_{\gamma}(B)}{(1 / 2)|B|}=2 \cdot o p t \mathrm{~N}_{\gamma}(s, t)
$$

Essa análise é justa no seguinte sentido. Para qualquer $\varepsilon>0$, vamos descrever uma matriz de pontuação e sequências para os quais o valor devolvido pelo algoritmo é pelo menos $(2-\varepsilon)$ vezes o valor da pontuação de um alinhamento N-ótimo. 
Sejam $\mathrm{a}, \mathrm{b} \in \Sigma \mathrm{e} \varepsilon>0$. Considere $\gamma$ a matriz de pontuação tal que

$$
\gamma_{\mathrm{a} \rightarrow \sqcup}=\gamma_{\iota \rightarrow \mathrm{b}}=\frac{1}{\varepsilon} \quad \text { e } \quad \gamma_{\mathrm{a} \rightarrow \mathrm{b}}=\frac{2}{\varepsilon}-1
$$

Considere também as sequências $\mathrm{a}^{n}, \mathrm{~b}^{n} \in \Sigma^{*}$, onde $n$ é um inteiro positivo. A pontuação de qualquer alinhamento de $\left(\mathrm{a}^{n}, \mathrm{~b}^{n}\right)$, onde [a, b] alinha em $k$ colunas é $2 n / \varepsilon-k$. Logo,

$$
\operatorname{opt} \mathrm{A}_{\gamma}\left(\mathrm{a}^{n}, \mathrm{~b}^{n}\right)=\min _{0 \leq k \leq n}\left\{\frac{2 n}{\varepsilon}-k\right\}=\frac{2 n}{\varepsilon}-n=\left(\frac{2}{\varepsilon}-1\right) n .
$$

Segue que $\left[\mathrm{a}^{n}, \mathrm{~b}^{n}\right]$ é o único alinhamento $A$-ótimo de $\left(\mathrm{a}^{n}, \mathrm{~b}^{n}\right)$. Além disso, a pontuação- $v \mathrm{~N}_{\gamma}$ do alinhamento $\left[\mathrm{a}^{n} \sqcup^{n}, \sqcup^{n} \mathrm{~b}^{n}\right]$ é $1 / \varepsilon$. Logo,

$$
\begin{aligned}
\frac{\operatorname{opt} \mathrm{A}_{\gamma}(s, t)}{\left|\left[\mathrm{a}^{n}, \mathrm{~b}^{n}\right]\right|} & =\frac{v \mathrm{~A}_{\gamma}\left(\left[\mathrm{a}^{n}, \mathrm{~b}^{n}\right]\right)}{\left|\left[\mathrm{a}^{n}, \mathrm{~b}^{n}\right]\right|} \\
& =\frac{(2 / \varepsilon-1) n}{n}=\frac{2}{\varepsilon}-1=(2-\varepsilon) \frac{1}{\varepsilon}=(2-\varepsilon) \cdot v \mathrm{~N}_{\gamma}\left(\left[\mathrm{a}^{n} \sqcup^{n},{ }^{n} \mathrm{~b}^{n}\right]\right) \\
& \geq(2-\varepsilon) \cdot \operatorname{opt} \mathrm{N}_{\gamma}\left(\mathrm{a}^{n}, \mathrm{~b}^{n}\right),
\end{aligned}
$$

o que significa que o valor devolvido pelo algoritmo é pelo menos $(2-\varepsilon)$ vezes o valor da pontuação do alinhamento N-ótimo.

\section{Algoritmo básico de Marzal e Vidal}

Marzal e Vidal [MV93] sugerem um algoritmo que usa uma tabela $d$ de programação dinâmica, armazenando na entrada $d(i, j, k)$, para cada $i=0,1, \ldots, n$, cada $j=0,1, \ldots, m$ e cada comprimento $k=\max \{i, j\}, \max \{i, j\}+1, \ldots, i+j$, a pontuação- $v \mathrm{~A}_{\gamma}$ de um alinhamento de $(s(1 \ldots i), t(1 \ldots j))$ de comprimento $k$ de menor pontuação- $v \mathrm{~A}_{\gamma}$.

Após computar a tabela $d$, o valor de um alinhamento N-ótimo é obtido de

$$
o p t \mathrm{~N}_{\gamma}(s, t)=\min _{k} \frac{d(n, m, k)}{k} .
$$

Esse algoritmo pode ser implementado gastando tempo e espaço $\Theta(n m(n+m))$.

\section{Pontuação- $v \mathrm{~A}_{\gamma}$ e pontuação- $v \mathrm{~N}_{\gamma}$}

A heurística descrita na seção anterior mostra implicitamente que um algoritmo para resolver o Problema APS não pode ser usado diretamente para calcular o valor de APSN. Nesta seção, vamos estabelecer uma relação entre a pontuação- $v \mathrm{~A}_{\gamma}$ e a pontuação- $v \mathrm{~N}_{\gamma}$ que permite descrever algoritmos mais rápidos para calcular opt $\mathrm{N}_{\gamma}$ usando algoritmos para o Problema APS. Para um par $(s, t)$ de sequências e um número real $\lambda$, a pontuação- $v \mathrm{~A}_{\gamma}(\lambda)$ de um alinhamento $A$ de $(s, t)$ é

$$
v \mathrm{~A}_{\gamma}(\lambda)(A)=v \mathrm{~A}_{\gamma}(A)-\lambda|A| .
$$

A função ótima para esse critério é

$$
\operatorname{opt} \mathrm{A}_{\gamma}(\lambda)(s, t)=\min _{A \in \mathcal{A}_{s, t}}\left\{v \mathrm{~A}_{\gamma}(\lambda)(A)\right\}
$$


e um alinhamento $\mathrm{A}(\lambda)$-ótimo de $(s, t)$ para $\gamma$ é um alinhamento cuja pontuação- $v \mathrm{~A}_{\gamma}(\lambda)$ é opt $\mathrm{A}_{\gamma}(\lambda)(s, t)$.

Note que um alinhamento $\mathrm{A}(0)$-ótimo para $\gamma$ é A-ótimo e que opt $\mathrm{A}_{\gamma}(0)(s, t)=o p t \mathrm{~A}_{\gamma}(s, t)$. Note também que opt $\mathrm{A}_{\gamma}(\lambda)(s, t)=o p t \mathrm{~A}_{\delta}(s, t)$, onde $\delta$ é a matriz de pontuação obtida subtraindo $\lambda$ de cada entrada da matriz $\gamma$. Portanto, um algoritmo para encontrar opt $\mathrm{A}_{\delta}(s, t)$ é um algoritmo para encontrar opt $\mathrm{A}_{\gamma}(\lambda)(s, t)$.

Os próximos lemas estabelecem relações entre os valores de $\operatorname{opt} \mathrm{A}_{\gamma}(\lambda)(s, t)$ e $\operatorname{opt} \mathrm{N}_{\gamma}(s, t)$.

Lema 5. Sejam $s, t \in \Sigma^{*}$ e $\lambda \in \mathbb{R}$. Então, $\lambda>\operatorname{opt} \mathrm{N}_{\gamma}(s, t)$ se e somente se $\operatorname{opt} \mathrm{A}_{\gamma}(\lambda)(s, t)<0$.

Prova. Vamos demonstrar o lema provando que se $\lambda>\operatorname{opt} \mathrm{N}_{\gamma}(s, t)$, então opt $\mathrm{A}_{\gamma}(\lambda)(s, t)<0$, e, depois, que se opt $\mathrm{A}_{\gamma}(\lambda)(s, t)<0$, então $\lambda>$ opt $\mathrm{N}_{\gamma}(s, t)$.

Suponha $\lambda>$ opt $\mathrm{N}_{\gamma}(s, t)$. Considere que $A$ é um alinhamento N-ótimo de $(s, t)$, ou seja, opt $\mathrm{N}_{\gamma}(s, t)=v \mathrm{~N}_{\gamma}(A)$. Segue que $\lambda>\operatorname{opt} \mathrm{N}_{\gamma}(s, t)=v \mathrm{~N}_{\gamma}(A)=v \mathrm{~A}_{\gamma}(A) /|A|$, o que implica que $\lambda>v \mathrm{~A}_{\gamma}(A) /|A|$. Logo, $v \mathrm{~A}_{\gamma}(A)-\lambda|A|<0$. Desde que opt $\mathrm{A}_{\gamma}(\lambda)(s, t) \leq v \mathrm{~A}_{\gamma}(\lambda)(A)$, segue que opt $\mathrm{A}_{\gamma}(\lambda)(s, t) \leq v \mathrm{~A}_{\gamma}(\lambda)(A)=v \mathrm{~A}_{\gamma}(A)-\lambda|A|<0$. Portanto, se $\lambda>\operatorname{opt} \mathrm{N}_{\gamma}(s, t)$, então $\operatorname{opt}_{\gamma}(\lambda)(s, t)<0$.

Reciprocamente, suponha que opt $\mathrm{A}_{\gamma}(\lambda)(s, t)<0$. Considere que $B$ é um alinhamento $\mathrm{A}(\lambda)$-ótimo de $(s, t)$ para $\gamma$. Segue que $v \mathrm{~A}_{\gamma}(B)-\lambda|B|=v \mathrm{~A}_{\gamma}(\lambda)(B)=\operatorname{opt}_{\gamma}(\lambda)(s, t)<0$. Logo, $v \mathrm{~A}_{\gamma}(B)-\lambda|B|<0$, o que implica que $\lambda>v \mathrm{~A}_{\gamma}(B) /|B|$. Sendo $v \mathrm{~A}_{\gamma}(B) /|B|=v \mathrm{~N}_{\gamma}(B)$ e $v \mathrm{~N}_{\gamma}(B) \geq \operatorname{opt} \mathrm{N}_{\gamma}(s, t)$, segue que $\lambda>v \mathrm{~A}_{\gamma}(B) /|B|=v \mathrm{~N}_{\gamma}(B) \geq \operatorname{opt} \mathrm{N}_{\gamma}(s, t)$. Portanto, se opt $\mathrm{A}_{\gamma}(\lambda)(s, t)<0$, então $\lambda>$ opt $\mathrm{N}_{\gamma}(s, t)$.

Lema 6. Sejam $s, t \in \Sigma^{*}$ e $\lambda \in \mathbb{R}$. Então, $\lambda=o p t \mathrm{~N}_{\gamma}(s, t)$ se e somente se $\operatorname{opt} \mathrm{A}_{\gamma}(\lambda)(s, t)=0$.

Prova. Vamos demonstrar o lema provando que se $\lambda=\operatorname{opt} \mathrm{N}_{\gamma}(s, t)$, então opt $\mathrm{A}_{\gamma}(\lambda)(s, t)=0$, e que se opt $\mathrm{A}_{\gamma}(\lambda)(s, t)=0$, então $\lambda=o p t \mathrm{~N}_{\gamma}(s, t)$.

Suponha então que $\lambda=o p t \mathrm{~N}_{\gamma}(s, t)$. Seja $A$ é um alinhamento N-ótimo de $(s, t)$. Segue que $\lambda=\operatorname{opt} \mathrm{N}_{\gamma}(s, t)=v \mathrm{~N}_{\gamma}(A)=v \mathrm{~A}_{\gamma}(A) /|A|$. Logo, $v \mathrm{~A}_{\gamma}(A)-\lambda|A|=0$. Desde que opt $\mathrm{A}_{\gamma}(\lambda)(s, t) \leq v \mathrm{~A}_{\gamma}(\lambda)(A)$ e $v \mathrm{~A}_{\gamma}(\lambda)(A)=v \mathrm{~A}_{\gamma}(A)-\lambda|A|$, segue que opt $\mathrm{A}_{\gamma}(\lambda)(s, t) \leq$ $v \mathrm{~A}_{\gamma}(\lambda)(A)=v \mathrm{~A}_{\gamma}(A)-\lambda|A|=0$. Logo opt $\mathrm{A}_{\gamma}(\lambda)(s, t)<0$ ou opt $\mathrm{A}_{\gamma}(\lambda)(s, t)=0$. Desde que opt $\mathrm{A}_{\gamma}(\lambda)(s, t)<0$ implica pelo Lema 5 que $\lambda>\operatorname{opt} \mathrm{N}_{\gamma}(s, t)$ contrariando a hipótese de que $\lambda=\operatorname{opt} \mathrm{N}_{\gamma}(s, t)$, segue que opt $\mathrm{A}_{\gamma}(\lambda)(s, t)=0$. Logo, se $\lambda=\operatorname{opt} \mathrm{N}_{\gamma}(s, t)$, então $\operatorname{optA}_{\gamma}(\lambda)(s, t)=0$.

Reciprocamente, suponha que opt $\mathrm{A}_{\gamma}(\lambda)(s, t)=0$. Seja $B$ é um alinhamento $\mathrm{A}(\lambda)$-ótimo de $(s, t)$ para $\gamma$. Segue que $v \mathrm{~A}_{\gamma}(B)-\lambda|B|=0$, o que implica que $\lambda=v \mathrm{~A}_{\gamma}(B) /|B|$. Segue que $\lambda=v \mathrm{~A}_{\gamma}(B) /|B|=v \mathrm{~N}_{\gamma}(B) \geq \operatorname{opt} \mathrm{N}_{\gamma}(s, t)$. Logo, $\lambda>\operatorname{opt} \mathrm{N}_{\gamma}(s, t)$ ou $\lambda=\operatorname{opt} \mathrm{N}_{\gamma}(s, t)$. Desde que $\lambda>\operatorname{opt} \mathrm{N}_{\gamma}(s, t)$ implica pelo Lema 5 que opt $\mathrm{A}_{\gamma}(\lambda)(s, t)<0$ contrariando a hipótese de que opt $\mathrm{A}_{\gamma}(\lambda)(s, t)=0$, segue que $\lambda=$ opt $\mathrm{N}_{\gamma}(s, t)$. Portanto, se opt $\mathrm{A}_{\gamma}(\lambda)(s, t)=0$, então $\lambda=\operatorname{opt} \mathrm{N}_{\gamma}(s, t)$.

Lema 7. Sejam $s, t \in \Sigma^{*}$ e $\lambda \in \mathbb{R}$. Então, $\lambda<o p t \mathrm{~N}_{\gamma}(s, t)$ se e somente se $\operatorname{opt} \mathrm{A}_{\gamma}(\lambda)(s, t)>0$.

Prova. Pelos Lemas 5 e 6 temos que $\lambda \ngtr o p t \mathrm{~N}_{\gamma}(s, t)$ se e somente se opt $\mathrm{A}_{\gamma}(\lambda)(s, t) \nless 0$; e $\lambda \neq o p t \mathrm{~N}_{\gamma}(s, t)$ se e somente se opt $\mathrm{A}_{\gamma}(\lambda)(s, t) \neq 0$. Portanto, $\lambda<$ opt $\mathrm{N}_{\gamma}(s, t)$ se e somente se $o p t \mathrm{~A}_{\gamma}(\lambda)(s, t)>0$.

Lema 8. Sejam $s, t \in \Sigma^{*}, \lambda=\operatorname{opt} \mathrm{N}_{\gamma}(s, t)$ e $A$ um alinhamento de $(s, t)$. Então, $A$ é um alinhamento $\mathrm{N}$-ótimo se e somente se $A$ é um alinhamento $\mathrm{A}(\lambda)$-ótimo. 
Prova. Vamos demonstrar o lema provando que se $A$ é um alinhamento N-ótimo, então $A$ é um alinhamento $\mathrm{A}(\lambda)$-ótimo, e que se $A$ é um alinhamento $\mathrm{A}(\lambda)$-ótimo, então $A$ é um alinhamento N-ótimo.

Suponha que $A$ é um alinhamento N-ótimo, ou seja, $v \mathrm{~A}_{\gamma}(A) /|A|=v \mathrm{~N}_{\gamma}(A)=\lambda$. Segue que $v \mathrm{~A}_{\gamma}(A)-\lambda|A|=0$. Seja $B$ um alinhamento arbitrário. Logo, $v \mathrm{~A}_{\gamma}(B) /|B|=v \mathrm{~N}_{\gamma}(B) \geq \lambda$. Segue que $v \mathrm{~A}_{\gamma}(B)-\lambda|B| \geq 0$. Segue que $v \mathrm{~A}_{\gamma}(\lambda)(A)=v \mathrm{~A}_{\gamma}(A)-\lambda|A|=0 \leq v \mathrm{~A}_{\gamma}(B)-\lambda|B|=$ $v \mathrm{~A}_{\gamma}(\lambda)(B)$. Sendo $B$ um alinhamento arbitrário, segue que $A$ é $\mathrm{A}(\lambda)$-ótimo. Portanto, se $A$ é um alinhamento N-ótimo, então $A$ é um alinhamento $\mathrm{A}(\lambda)$-ótimo.

Reciprocamente, suponha que $A$ é $\mathrm{A}(\lambda)$-ótimo, ou seja, $v \mathrm{~A}_{\gamma}(A)-\lambda|A|=\operatorname{opt} \mathrm{A}_{\gamma}(\lambda)(s, t)$. Como $\lambda=\operatorname{opt} \mathrm{N}_{\gamma}(s, t)$, temos pelo Lema 6 que opt $\mathrm{A}_{\gamma}(\lambda)(s, t)=0$. Logo, $v \mathrm{~A}_{\gamma}(A)-\lambda|A|=$ opt $\mathrm{A}_{\gamma}(\lambda)(s, t)=0$, o que implica, sendo $\lambda=\operatorname{opt} \mathrm{N}_{\gamma}(s, t)$ e $v \mathrm{~A}_{\gamma}(A) /|A|=\lambda$, que $A$ é N-ótimo. Portanto, se $A$ é um alinhamento $\mathrm{A}(\lambda)$-ótimo, então $A$ é um alinhamento N-ótimo.

\section{Algoritmo desenvolvido por Vidal, Marzal e Aibar [VMA95]}

Vidal, Marzal e Aibar [VMA95], baseados nos conceitos apresentados na seção anterior descrevem um outro método, que é o Algoritmo 1, para determinar o valor de opt $\mathrm{N}_{\gamma}(s, t)$ usando um algoritmo para encontrar valores de $\operatorname{opt} \mathrm{A}_{\gamma}(\lambda)(s, t)$ para diversos valores de $\lambda$.

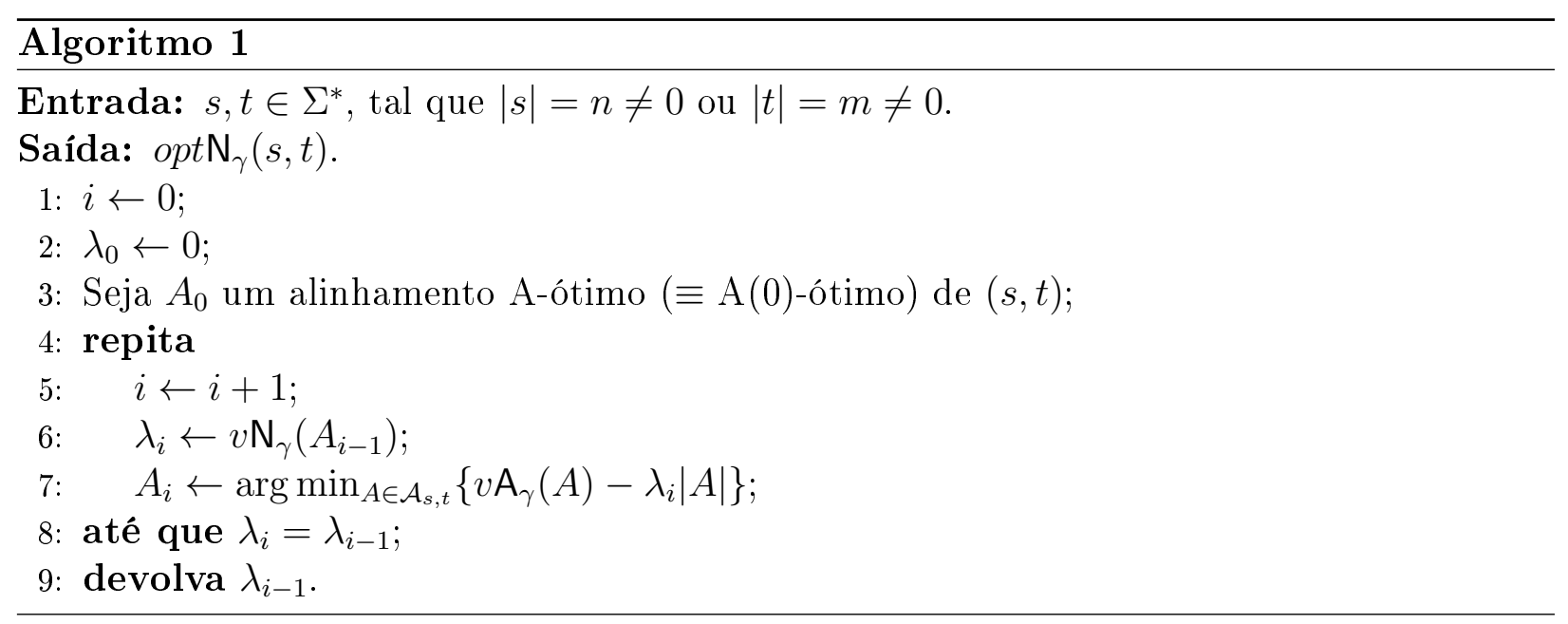

Verificamos o seguinte invariante do Algoritmo 1.

Proposição 9. Para $i \geq 2$, vale que $\lambda_{i-1} \geq \lambda_{i}$.

Prova. Seja $i \geq 2$. Pela Linha 6 do Algoritmo 1 , temos que $\lambda_{i-1}=v \mathrm{~A}_{\gamma}\left(A_{i-2}\right) /\left|A_{i-2}\right|$. Logo, $v \mathrm{~A}_{\gamma}\left(A_{i-2}\right)-\lambda_{i-1}\left|A_{i-2}\right|=0$. Pela minimalidade de $A_{i-1}$ calculada na Linha 7 , temos que $v \mathrm{~A}_{\gamma}\left(A_{i-1}\right)-\lambda_{i-1}\left|A_{i-1}\right| \leq v \mathrm{~A}_{\gamma}\left(A_{i-2}\right)-\lambda_{i-1}\left|A_{i-2}\right|$. Segue que

$$
v \mathrm{~A}_{\gamma}\left(A_{i-1}\right)-\lambda_{i-1}\left|A_{i-1}\right| \leq v \mathrm{~A}_{\gamma}\left(A_{i-2}\right)-\lambda_{i-1}\left|A_{i-2}\right|=0,
$$

o que implica que $\lambda_{i-1} \geq v \mathrm{~A}_{\gamma}\left(A_{i-1}\right) /\left|A_{i-1}\right|$. Sendo, pela Linha $6, \lambda_{i}=v \mathrm{~A}_{\gamma}\left(A_{i-1}\right) /\left|A_{i-1}\right|$, segue que $\lambda_{i-1} \geq v \mathrm{~A}_{\gamma}\left(A_{i-1}\right) /\left|A_{i-1}\right|=\lambda_{i}$. Portanto, para $i \geq 2$, vale que $\lambda_{i-1} \geq \lambda_{i}$.

Proposição 10. Se o Algoritmo 1 executa $Q$ iterações, então, para cada par de inteiro $p, q$ com $0 \leq p<q<Q-1$, temos que $\left|A_{p}\right| \neq\left|A_{q}\right|$. 
Prova. Suponha que Algoritmo 1 executa $Q$ iterações e que $p$ e $q$ são inteiros não negativos tais que $p<q<Q-1$ e, por contradição, $\left|A_{p}\right|=\left|A_{q}\right|$. Sendo $p<q<Q-1$, segue que $p+2<Q$. Pela Linha 7 temos que

$$
v \mathrm{~A}_{\gamma}\left(A_{p}\right)-\lambda_{p}\left|A_{p}\right| \leq v \mathrm{~A}_{\gamma}\left(A_{q}\right)-\lambda_{p}\left|A_{q}\right| \quad \text { e } \quad v \mathrm{~A}_{\gamma}\left(A_{q}\right)-\lambda_{q}\left|A_{q}\right| \leq v \mathrm{~A}_{\gamma}\left(A_{p}\right)-\lambda_{q}\left|A_{p}\right|,
$$

o que implica, sendo $\left|A_{p}\right|=\left|A_{q}\right|$, que $v \mathrm{~A}_{\gamma}\left(A_{p}\right) \leq v \mathrm{~A}_{\gamma}\left(A_{q}\right)$ e $v \mathrm{~A}_{\gamma}\left(A_{q}\right) \leq v \mathrm{~A}_{\gamma}\left(A_{p}\right)$, respectivamente. $\log 0 v \mathrm{~A}_{\gamma}\left(A_{p}\right)=v \mathrm{~A}_{\gamma}\left(A_{q}\right)$. Segue que

$$
\lambda_{p+1}=\frac{v \mathrm{~A}_{\gamma}\left(A_{p}\right)}{\left|A_{p}\right|}=\frac{v \mathrm{~A}_{\gamma}\left(A_{q}\right)}{\left|A_{q}\right|}=\lambda_{q+1} .
$$

Sendo $p<q<Q-1$, segue da Proposição 9 que $\lambda_{p+1}=\lambda_{p+2}=\ldots=\lambda_{q}=\lambda_{q+1}$. Logo, pela Linha 8 , o algoritmo para, no máximo, na iteração $p+2$ contrariando que $p+2<Q$. Portanto, se o algoritmo executa $Q$ iterações, então $\left|A_{p}\right| \neq\left|A_{q}\right|$ para cada par de inteiro $p, q$ com $0 \leq p<q<Q-1$.

Lema 11. Depois de no máximo $\min \{n, m\}+2$ iterações, o Algoritmo 1 para.

Prova. Suponha por contradição que o Algoritmo 1 executa mais do que $\min \{n, m\}+2$ iterações. Sejam $A_{0}, \ldots, A_{\min \{n, m\}+3}$ alinhamentos que essa hipótese garante existir. Pelo Fato 1, temos $\max \{n, m\} \leq\left|A_{i}\right| \leq n+m$ para cada $A_{i}$, o que implica que existem $\min \{n, m\}+1=(n+m)-\max \{n, m\}+1$ comprimentos possíveis para o alinhamento $A_{i}$. Logo, há pelo menos dois alinhamentos com mesmo comprimento em $A_{0}, \ldots, A_{\min \{n, m\}+1}$ contrariando a Proposição 10. Portanto, depois de no máximo $\min \{n, m\}+2$ iterações, o Algoritmo 1 para.

Lema 12. Se $\lambda_{i}=\lambda_{i-1}$, então $\lambda_{i-1}=\operatorname{opt} \mathrm{N}_{\gamma}(s, t)$.

Prova. Suponha que $\lambda_{i}=\lambda_{i-1}$. Pela execução da Linha 7 na iteração $i-1$ temos que $A_{i-1}$ é um alinhamento $\mathrm{A}\left(\lambda_{i-1}\right)$-ótimo, ou seja, opt $\mathrm{A}_{\gamma}\left(\lambda_{i-1}\right)(s, t)=v \mathrm{~A}_{\gamma}\left(A_{i-1}\right)-\lambda_{i-1}\left|A_{i-1}\right|$. Pela Linha 6 , temos que $\lambda_{i}=v \mathrm{~N}_{\gamma}\left(A_{i-1}\right)$, ou seja, $\lambda_{i}=v \mathrm{~A}_{\gamma}\left(A_{i-1}\right) /\left|A_{i-1}\right|$. Logo, $v \mathrm{~A}_{\gamma}\left(A_{i-1}\right)-$ $\lambda_{i}\left|A_{i-1}\right|=0$. Pela hipótese de que $\lambda_{i}=\lambda_{i-1}$ segue que

$$
\operatorname{optA}_{\gamma}\left(\lambda_{i-1}\right)(s, t)=v \mathrm{~A}_{\gamma}\left(A_{i-1}\right)-\lambda_{i-1}\left|A_{i-1}\right|=v \mathrm{~A}_{\gamma}\left(A_{i-1}\right)-\lambda_{i}\left|A_{i-1}\right|=0 .
$$

Segue do Lema 6 que $\lambda_{i-1}=\operatorname{opt} \mathrm{N}_{\gamma}(s, t)$. Portanto, se $\lambda_{i}=\lambda_{i-1}$, então $\lambda_{i-1}=\operatorname{opt} \mathrm{N}_{\gamma}(s, t)$.

O Lema 11 garante que Algoritmo 1 para e o Lema 12 garante que, quando para, o valor devolvido é opt $\mathrm{N}_{\gamma}(s, t)$ conforme esperado. Portanto, o algoritmo está correto.

O Lema 11 garante que Algoritmo 1 faz no máximo $\min \{n, m\}+2$ iterações. Logo, se $n=m$, então o tempo gasto no pior caso é $O\left(T_{\mathrm{APS}}(n) \cdot n\right)$, onde, lembramos, $T_{\mathrm{APS}}(n)$ é o tempo gasto no pior caso para encontrar a pontuação e um um alinhamento A-ótimo para $(s, t)$ para o critério $v \mathrm{~A}_{\delta}$, onde $\delta$ é a matriz obtida subtraindo $\lambda$ de cada entrada de $\gamma$. Os autores enfatizam que, embora $T_{\mathrm{APS}}(n)=O\left(n^{2}\right)$ e, portanto, $O\left(T_{\mathrm{APS}}(n) \cdot n\right)=O\left(n^{3}\right)$, na prática, os testes por eles realizados mostram que o algoritmo é muito mais rápido que isto, sugerindo que essa análise esteja superestimada. Entretanto, não há provas de que de fato seja esse o caso.

\section{Algoritmo de Arslan e Egecioglu [AE00]}

Assim como o algoritmo estudado na seção anterior, o algoritmo descrito por Arslan e Egecioglu [AE00] computa alinhamentos $\mathrm{A}(\lambda)$-ótimos até encontrar um $\lambda=o p t \mathrm{~N}_{\gamma}(s, t)$ 
quando $\min _{A \in \mathcal{A}_{s, t}}\left\{v \mathrm{~A}_{\gamma}(\lambda)(A)\right\}=0$. Entretanto, ele usa uma estratégia de busca binária para encontrar o $\lambda$ correto obtendo um gasto de tempo garantidamente mais rápido que o método anterior. A restrição para obter essa melhora no tempo gasto é de que a matriz de pontuação tenha entradas racionais, o que parece não ser na prática uma restrição muito grande. Por simplicidade, consideramos aqui que as entradas da matriz de pontuação são números inteiros.

Lembramos que, conforme nossa convenção, $\gamma_{\max }$ e $\gamma_{\min }$ são os valores da maior e da menor entrada em $\gamma$.

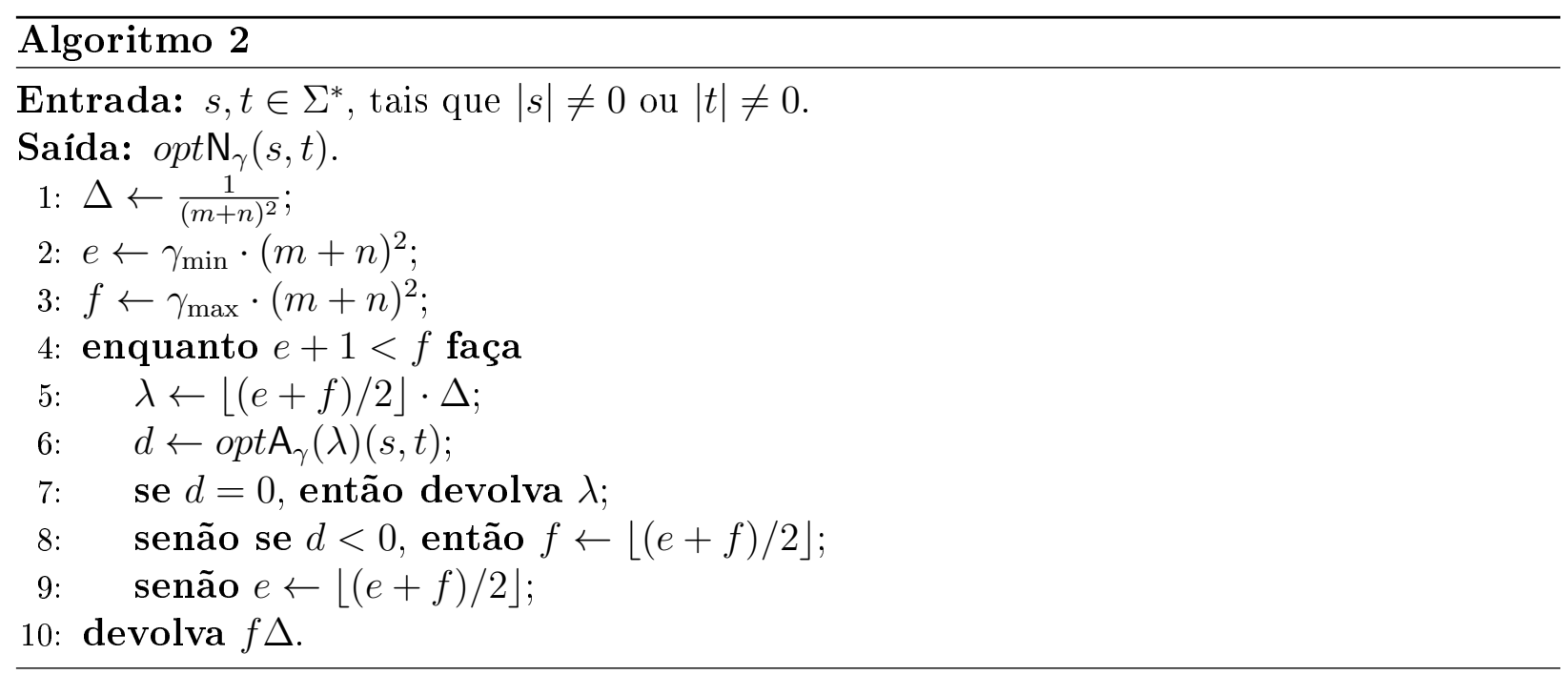

Os resultados a seguir auxiliam na análise da correção e do tempo gasto do Algoritmo 2.

Proposição 13. Sejam $\gamma$ uma matriz de pontuação com entradas inteiras, $s, t \in \Sigma^{*}$ com comprimentos $n$ e $m$, respectivamente, e $A$ e $B$ alinhamentos de $(s, t)$. Se $v \mathrm{~N}_{\gamma}(A) \neq v \mathrm{~N}_{\gamma}(B)$, então

$$
\left|v \mathrm{~N}_{\gamma}(A)-v \mathrm{~N}_{\gamma}(B)\right|>\frac{1}{(n+m)^{2}}
$$

Prova. Suponha que $v \mathrm{~N}_{\gamma}(A) \neq v \mathrm{~N}_{\gamma}(B)$. Logo, $\left|v \mathrm{~A}_{\gamma}(A)\right| B\left|-v \mathrm{~A}_{\gamma}(B)\right| A \|>0$. Desde que $\gamma$ é uma matriz de pontuação com entradas inteiras, segue que $\left|v \mathrm{~A}_{\gamma}(A)\right| B\left|-v \mathrm{~A}_{\gamma}(B)\right| A|| \geq 1$. Pelo Fato 1 , temos que $|A| \leq n+m$ e $|B| \leq n+m$. e, desde que $\left|v \mathrm{~A}_{\gamma}(A)\right| B\left|-v \mathrm{~A}_{\gamma}(B)\right| A||>0$, $A$ e $B$ não podem ter ao mesmo tempo comprimento $n+m$, o que implica que $|A||B|<(n+m)^{2}$. Segue que

$$
\begin{aligned}
\left|v \mathrm{~N}_{\gamma}(A)-v \mathrm{~N}_{\gamma}(B)\right| & =\left|\frac{v \mathrm{~A}_{\gamma}(A)}{|A|}-\frac{v \mathrm{~A}_{\gamma}(B)}{|B|}\right|=\frac{\left|v \mathrm{~A}_{\gamma}(A)\right| B\left|-v \mathrm{~A}_{\gamma}(B)\right| A||}{|A||B|} \geq \frac{1}{|A||B|} \\
& >\frac{1}{(n+m)^{2}} .
\end{aligned}
$$

Lema 14. No início de cada iteração do Algoritmo 2 (imediatamente antes da Linha 4 ser executada), vale $e \Delta \leq$ opt $\mathrm{N}_{\gamma}(s, t) \leq f \Delta$.

Prova. No início da primeira iteração, $e \Delta=\gamma_{\min }$ e $f \Delta=\gamma_{\max }$. Pelo Fato 4 , temos que $\gamma_{\min } \leq v \mathrm{~N}_{\gamma}(A) \leq \gamma_{\max }$ para um alinhamento N-ótimo $A$ de $(s, t)$. Segue que $e \Delta=\gamma_{\min } \leq$ $v \mathrm{~N}_{\gamma}(A)=\operatorname{opt} \mathrm{N}_{\gamma}(s, t) \leq \gamma_{\max }=f \Delta$. Portanto, vale o invariante no início da primeira iteração. 
Suponha que estamos no início de uma iteração qualquer que não é a última e que vale, nesse momento, $e \Delta \leq o p t \mathrm{~N}_{\gamma}(s, t) \leq f \Delta$. Nesta iteração, o algoritmo calcula um novo valor para $\lambda$ e para $d$ executando as Linhas 5 e 6 . Note que $d \neq 0$ porque não estamos na última iteração, o que implica que a Linha 7 não é executada e que $d<0$ ou $d>0$. Se $d<0$, então pelo Lema 5 temos que opt $\mathrm{N}_{\gamma}(s, t)<\lambda$. Logo, e $\Delta \leq \operatorname{opt} \mathrm{N}_{\gamma}(s, t) \leq \lambda=\lfloor(e+f) / 2\rfloor \cdot \Delta$ e, portanto, sendo $f$ atualizado na Linha 8 , vale o invariante no início da próxima iteração. Se $d>0$, então segue do Lema 7 que $\lfloor(e+f) / 2\rfloor \cdot \Delta=\lambda \leq o p t \mathrm{~N}_{\gamma}(s, t) \leq f$ e, portanto, sendo $e$ atualizado na Linha 9 , também vale o invariante no início da próxima iteração.

Lema 15. Sejam $\gamma$ uma matriz de pontuação com entradas inteiras, $s, t \in \Sigma^{*}$ tais que $|s|=n$ e $|t|=m$, e $\lambda_{1}, \lambda_{2}$ números reais tais que $\lambda_{1} \leq \operatorname{opt}_{\gamma}(s, t) \leq \lambda_{2}$ e $\lambda_{2}-\lambda_{1} \leq 1 /(n+m)^{2}$. Então, um alinhamento $\mathrm{A}\left(\lambda_{2}\right)$-ótimo de $(s, t)$ é N-ótimo.

Prova. Suponha que $A$ é um alinhamento N-ótimo e $B$ um alinhamento $\mathrm{A}\left(\lambda_{2}\right)$-ótimo. Como $\operatorname{opt} \mathrm{N}_{\gamma}(s, t)=v \mathrm{~N}_{\gamma}(A)$ e $\lambda_{1} \leq \operatorname{opt} \mathrm{N}_{\gamma}(s, t)$, temos que $\lambda_{1} \leq v \mathrm{~N}_{\gamma}(A)$. Como opt $\mathrm{N}_{\gamma}(s, t) \leq \lambda_{2}$, pelos Lemas 5 e 6 temos que opt $\mathrm{A}_{\gamma}\left(\lambda_{2}\right)(s, t) \leq 0$ e, desde que $v \mathrm{~A}_{\gamma}\left(\lambda_{2}\right)(B)=o p t \mathrm{~A}_{\gamma}\left(\lambda_{2}\right)(s, t)$, segue que $v \mathrm{~A}_{\gamma}(B)-\lambda_{2}|B|=v \mathrm{~A}_{\gamma}\left(\lambda_{2}\right)(B) \leq 0$, o que implica que $v \mathrm{~N}_{\gamma}(B)=v \mathrm{~A}_{\gamma}(B) /|B| \leq \lambda_{2}$. Como $A$ é um alinhamento N-ótimo, temos que $v \mathrm{~N}_{\gamma}(A) \leq v \mathrm{~N}_{\gamma}(B)$, e sendo $\lambda_{1} \leq v \mathrm{~N}_{\gamma}(A)$ e $v \mathrm{~N}_{\gamma}(B) \leq \lambda_{2}$, segue que $\left|v \mathrm{~N}_{\gamma}(B)-v \mathrm{~N}_{\gamma}(A)\right|=v \mathrm{~N}_{\gamma}(B)-v \mathrm{~N}_{\gamma}(A) \leq \lambda_{2}-\lambda_{1} \leq 1 /(n+m)^{2}$, o que implica, pela contrapositiva da Proposição 13, que $v \mathrm{~N}_{\gamma}(A)=v \mathrm{~N}_{\gamma}(B)$. Logo, o alinhamento $B$ é N-ótimo. Portanto, um alinhamento $\mathrm{A}\left(\lambda_{2}\right)$-ótimo de $(s, t)$ é N-ótimo.

Claramente o algoritmo para. Se o algoritmo para na Linha 7, então o Lema 6 garante que o valor devolvido pelo algoritmo é $\operatorname{opt} \mathrm{N}_{\gamma}(s, t)$. Suponha que o algoritmo para na Linha 10 . Como $f-e \leq 1$ nesse momento, temos que $f \Delta-e \Delta \leq \Delta=1 /(n+m)^{2}$. Pelo Lema 14, vale $e \Delta \leq \operatorname{opt} \mathrm{N}_{\gamma}(s, t) \leq f \Delta$ no início de cada iteração, o que implica que, em particular, vale no início da última iteração e, portanto, vale quando o algoritmo executa a Linha 10. Segue do Lema 15 que o alinhamento $\mathrm{A}(f \Delta)$-ótimo computado nesse momento é também um alinhamento N-ótimo. Portanto, na Linha 10 o algoritmo devolve corretamente opt $\mathrm{N}_{\gamma}(s, t)$.

Considerando $|s|=|t|=n$, o tempo gasto é limitado pelo número de iterações vezes $T_{\mathrm{APS}}(n)$ que é o tempo gasto no pior caso cada vez que a Linha 6 é executada pelo Algoritmo 2. O intervalo $[e, f]$ inicialmente igual a $\left[\gamma_{\min } \cdot(n+m)^{2}, \gamma_{\max } \cdot(n+m)^{2}\right]=$ $\left[\gamma_{\min } \cdot 4 n^{2}, \gamma_{\max } \cdot 4 n^{2}\right]$ é reduzido pela metade em cada iteração até ficar menor que 1 no pior caso. Portanto, a quantidade de iterações do algoritmo é, no máximo,

$$
\log \left(\gamma_{\max } \cdot 4 n^{2}-\gamma_{\min } \cdot 4 n^{2}\right)=2+2 \log n+\log \left(\gamma_{\max }-\gamma_{\min }\right)=\Theta(\log n),
$$

pois $\gamma_{\max }$ e $\gamma_{\min }$ são constantes. Segue que o tempo total gasto é $O\left(T_{\mathrm{APS}}(n) \cdot \log n\right)$.

\section{Alinhamentos com custo uniforme para inserção e remoção}

Uma das propostas de nosso doutorado foi pesquisar um algoritmo para determinar a pontuação de um alinhamento N-ótimo de duas sequências que fosse mais rápido do que os anteriormente descritos. Elaboramos um algoritmo para o problema que, entretanto, não é mais eficiente do que o Algoritmo 2, que é o algoritmo comprovadamente mais eficiente conhecido. Além disso, nosso algoritmo é menos geral porque ele só pode ser aplicado se o custo para inserção e remoção de símbolos é uniforme. Embora, em termos de resultado, o algoritmo não representa uma contribuição, julgamos interessante incluí-lo nesta tese pelo fato das estruturas usadas pelo algoritmo serem diferentes. Assim como os Algoritmos $1 \mathrm{e}$ 2 , nosso algoritmo calcula o valor de $\operatorname{opt}_{\gamma}(s, t)$ para diversos valores de $\gamma$, mas, em vez de, 
em cada iteração, alterar o valor de todas as entradas da matriz de pontuação, somente o valor do custo de inserção/remoção é alterado.

Consideramos na descrição do algoritmo que as entradas em $\gamma$ são números inteiros. Isto não representa uma restrição em relação às matrizes de pontuação que têm números racionais como entrada, dado que podemos obter uma matriz de pontuação de números inteiros equivalente a partir de uma matriz de pontuação com entradas racionais, multiplicando todas as entradas de $\gamma$ pelo mínimo múltiplo comum dos denominadores das entradas de $\gamma$. Essa transformação é feita em tempo constante se a matriz de pontuação não é parte da entrada do problema.

Considere $N=|s|+|t|$. A soma dos custos das operações de edição de substituição de um alinhamento $A$ é denotado por $c_{\gamma}(A)$. Então, o custo de inserção/remoção em $A$ é o valor $v \mathrm{~A}_{\gamma}(A)-c_{\gamma}(A)$. A quantidade total de operações de inserção e remoção em $A$ é denotada por $k(A)$. Se o custo de inserção e de remoção em $\gamma$ é uniforme e é $g$, então o custo de inserção/remoção em $A$ é $g k(A)$. Logo, nesse caso, $v \mathrm{~A}_{\gamma}(A)-c_{\gamma}(A)=g k(A)$, o que implica que $v \mathrm{~A}_{\gamma}(A)=c_{\gamma}(A)+g k(A)$. Note que o comprimento do alinhamento $A$ é $|A|=(N+k(A)) / 2$ e que a quantidade de operações de substituição é $(N-k(A)) / 2$. Dizemos que o par de inteiros $\left(c_{\gamma}(A), k(A)\right)$ corresponde ao alinhamento $A$. Para as sequências $s, t$, definimos o conjunto

$$
\mathbb{A}=\left\{\left(c_{\gamma}(A), k(A)\right): A \text { é um alinhamento em } \mathcal{A}_{s, t}\right\} .
$$

Se $g$ é o custo de inserção/remoção, determinar $\operatorname{optA}_{\gamma}(s, t)$ significa determinar o valor de $\min _{\left(c_{\gamma}(A), k(A)\right) \in \mathbb{A}}\left\{c_{\gamma}(A)+g k(A)\right\}$. Por simplicidade, escrevemos $(c, k)$ em vez de $\left(c_{\gamma}(A), k(A)\right)$ quando o alinhamento $A$ e a matriz de pontuação estiverem subentendidas. Dizemos que o custo de $(c, k)$ para $g$ é $c+g k$. Dizemos que $(c, k)$ é A-ótimo para $g$ e que $g$ define o par $(c, k) \in \mathbb{A}$ quando, para cada $\left(c^{\prime}, k^{\prime}\right) \in \mathbb{A}$, temos $c+g k \leq c^{\prime}+g k^{\prime}$ e, para $k^{\prime}>k$, temos $c+g k<c^{\prime}+g k^{\prime}$. Dizemos que $(c, k)$ é A-ótimo se $(c, k)$ é A-ótimo para algum $g$.

As seguintes observações estabelecem um limitante superior para a quantidade de espaços em um alinhamento:

(i) o par de inteiros $(0, N)$ corresponde a um alinhamento de comprimento máximo;

(ii) a quantidade de espaços em um alinhamento é no máximo $N$, ou seja, $k \leq N$ para qualquer $(c, k) \in \mathbb{A}$.

O seguinte resultado mostra uma relação entre $(c, k)$ e o comprimento do alinhamento que a ele corresponde.

Fato 16. Sejam $(c, k)$ e $\left(c^{\prime}, k^{\prime}\right)$ correspondentes aos alinhamentos $A$ e $A^{\prime}$ respectivamente. Então, valem:

(i) $|A| \leq\left|A^{\prime}\right|$ se e somente se $k \leq k^{\prime}$ e

(ii) se $|A|<\left|A^{\prime}\right|$, então $k \leq k^{\prime}-2$.

Prova. Sejam $(c, k)$ e $\left(c^{\prime}, k^{\prime}\right)$ correspondentes aos alinhamentos $A$ e $A^{\prime}$ respectivamente.

Como $|A|=(N+k) / 2$ e $\left|A^{\prime}\right|=\left(N+k^{\prime}\right) / 2$, temos que $|A| \leq\left|A^{\prime}\right|$ se e somente se $k \leq k^{\prime}$. Logo, vale $(i)$.

Suponha que $|A|<\left|A^{\prime}\right|$. Como $|A|,\left|A^{\prime}\right| \in \mathbb{Z}$, segue que $|A| \leq\left|A^{\prime}\right|-1$. Logo,

$$
\frac{N+k}{2}=|A| \leq\left|A^{\prime}\right|-1=\frac{N+k^{\prime}}{2}-1=\frac{N+k^{\prime}-2}{2}
$$


o que implica que $k \leq k^{\prime}-2$. Portanto, se $|A|<\left|A^{\prime}\right|$, então $k \leq k^{\prime}-2$. Logo, vale $(i i)$.

O seguinte resultado mostra que, para cada $k$, existe no máximo um elemento $(c, k)$ em $\mathbb{A}$ que é A-ótimo.

Fato 17. Sejam $(c, k),\left(c^{\prime}, k\right) \in \mathbb{A}$ e A-ótimos. Então $c=c^{\prime}$.

Prova. Como $(c, k),\left(c^{\prime}, k\right)$ são A-ótimos, existem $g$ e $g^{\prime}$ tais que $c+g k \leq c^{\prime}+g k$ e $c^{\prime}+g^{\prime} k \leq$ $c+g^{\prime} k$, respectivamente. Segue da primeira desigualdade que $c \leq c^{\prime}$ e da segunda desigualdade que $c^{\prime} \leq c$. Logo, $c=c^{\prime}$.

Definimos o conjunto $\mathcal{H}$ dos elementos A-ótimos em $\mathbb{A}$. Em outras palavras,

$$
\mathcal{H}=\{(c, k):(c, k) \text { é A-ótimo }\} \text {. }
$$

Se $\left(c_{1}, k_{1}\right),\left(c_{2}, k_{2}\right) \in \mathcal{H}$, então existem $g_{1}, g_{2}$ tais que $\left(c_{1}, k_{1}\right)$ é A-ótimo para $g_{1}$ e $\left(c_{2}, k_{2}\right)$ é A-ótimo para $g_{2}$. O próximo resultado mostra uma relação entre $g_{1}, g_{2}$ quando $k_{1}>k_{2}$.

Fato 18. Sejam $\left(c_{1}, k_{1}\right),\left(c_{2}, k_{2}\right)$ A-ótimos para $g_{1}$ e $g_{2}$ respectivamente. Se $k_{1}>k_{2}$, então

$$
g_{1} \leq \frac{c_{2}-c_{1}}{k_{1}-k_{2}}<g_{2}
$$

Prova. Suponha que $k_{1}>k_{2}$. Como $\left(c_{1}, k_{1}\right),\left(c_{2}, k_{2}\right)$ são A-ótimos para $g_{1}$ e $g_{2}$, temos que $c_{1}+g_{1} k_{1} \leq c_{2}+g_{1} k_{2}$ e $c_{2}+g_{2} k_{2}<c_{1}+g_{2} k_{1}$. Logo, sendo $k_{1}>k_{2}, g_{1} \leq\left(c_{2}-c_{1}\right) /\left(k_{1}-k_{2}\right)$ e $\left(c_{2}-c_{1}\right) /\left(k_{1}-k_{2}\right)<g_{2}$. Portanto, se $k_{1}>k_{2}$, então

$$
g_{1} \leq \frac{c_{2}-c_{1}}{k_{1}-k_{2}}<g_{2}
$$

Estendendo o conceito do conjunto $\mathcal{H}$, definimos

$$
\mathcal{H}\left[g_{1}, g_{2}\right]=\left\{(c, k) \in \mathbb{A}:(c, k) \text { é A-ótimo para } g, g_{1} \leq g \leq g_{2}\right\} .
$$

Note que, para quaisquer $g_{1}, g_{2}$, temos que

$$
\mathcal{H}\left[g_{1}, g_{2}\right] \subseteq \mathcal{H}
$$

Se $(c, k) \in \mathbb{A}$ é o par correspondente a um alinhamento $A$ qualquer, então $(N-k) / 2$ é a quantidade de substituições em $A$. Desde que o custo individual de cada operação de substituição não é inferior a $\min _{a, b \in \Sigma}\left\{\gamma_{a \rightarrow b}\right\}$ nem superior a $\max _{a, b \in \Sigma}\left\{\gamma_{a \rightarrow b}\right\}$, segue que

$$
\frac{N-k}{2} \min _{\mathrm{a}, \mathrm{b} \in \Sigma}\left\{\gamma_{\mathrm{a} \rightarrow \mathrm{b}}\right\} \leq c \leq \frac{N-k}{2} \max _{\mathrm{a}, \mathrm{b} \in \Sigma}\left\{\gamma_{\mathrm{a} \rightarrow \mathrm{b}}\right\}
$$

Os resultados a seguir mostram valores para $g_{1}$ e $g_{2}$ de modo que $\mathcal{H}=\mathcal{H}\left[g_{1}, g_{2}\right]$.

Proposição 19. O par $(0, N)$ é A-ótimo para $\min _{\mathrm{a}, \mathrm{b} \in \Sigma}\left\{\gamma_{\mathrm{a} \rightarrow \mathrm{b}}\right\} / 2$.

Prova. Para cada $\left(c^{\prime}, k^{\prime}\right) \in \mathbb{A}$, temos que

$$
\begin{aligned}
0+\frac{\min _{\mathrm{a}, \mathrm{b} \in \Sigma}\left\{\gamma_{\mathrm{a} \rightarrow \mathrm{b}}\right\}}{2} N & =\frac{\min _{\mathrm{a}, \mathrm{b} \in \Sigma}\left\{\gamma_{\mathrm{a} \rightarrow \mathrm{b}}\right\}}{2}\left(N-k^{\prime}\right)+\frac{\min _{\mathrm{a}, \mathrm{b} \in \Sigma}\left\{\gamma_{\mathrm{a} \rightarrow \mathrm{b}}\right\}}{2} k^{\prime} \\
& \leq c^{\prime}+\frac{\min _{\mathrm{a}, \mathrm{b} \in \Sigma}\left\{\gamma_{\mathrm{a} \rightarrow \mathrm{b}}\right\}}{2} k^{\prime}
\end{aligned}
$$


onde a desigualdade segue de (2.5). Desde que $N \geq k^{\prime}$, para cada $\left(c^{\prime}, k^{\prime}\right) \in \mathbb{A}$, segue que $(0, N)$ é A-ótimo para $\min _{\mathrm{a}, \mathrm{b} \in \Sigma}\left\{\gamma_{\mathrm{a} \rightarrow \mathrm{b}}\right\} / 2$.

Fato 20. Se $g>-N \cdot \min _{\mathrm{a}, \mathrm{b} \in \Sigma}\left\{\gamma_{\mathrm{a} \rightarrow \mathrm{b}}\right\}$ e $g>0$, então

$$
-g<\frac{N-k}{2} \min _{\mathrm{a}, \mathrm{b} \in \Sigma}\left\{\gamma_{\mathrm{a} \rightarrow \mathrm{b}}\right\}
$$

para cada $(c, k) \in \mathbb{A}$.

Prova. Suponha que $g>-N \cdot \min _{\mathrm{a}, \mathrm{b} \in \Sigma}\left\{\gamma_{\mathrm{a} \rightarrow \mathrm{b}}\right\}$ e $g>0$. Se $\min _{\mathrm{a}, \mathrm{b} \in \Sigma}\left\{\gamma_{\mathrm{a} \rightarrow \mathrm{b}}\right\} \geq 0$, então, desde que $N \geq k$ e $g>0$

$$
-g<0 \leq \frac{N-k}{2} \min _{\mathrm{a}, \mathrm{b} \in \Sigma}\left\{\gamma_{\mathrm{a} \rightarrow \mathrm{b}}\right\}
$$

e o fato está provado; se $\min _{\mathrm{a}, \mathrm{b} \in \Sigma}\left\{\gamma_{\mathrm{a} \rightarrow \mathrm{b}}\right\}<0$, desde que $N \geq k$ e $g>-N \cdot \min _{\mathrm{a}, \mathrm{b} \in \Sigma}\left\{\gamma_{\mathrm{a} \rightarrow \mathrm{b}}\right\}$,

$$
-g<N \cdot \min _{\mathrm{a}, \mathrm{b} \in \Sigma}\left\{\gamma_{\mathrm{a} \rightarrow \mathrm{b}}\right\} \leq \frac{N-k}{2} \min _{\mathrm{a}, \mathrm{b} \in \Sigma}\left\{\gamma_{\mathrm{a} \rightarrow \mathrm{b}}\right\}
$$

e também para este caso o fato está provado.

Proposição 21. Seja $(c, k) \in \mathbb{A}$ o par que corresponde a um alinhamento de menor pontuação entre os alinhamentos de comprimento mínimo e

$$
g>N \cdot \max \left\{-\min _{\mathrm{a}, \mathrm{b} \in \Sigma}\left\{\gamma_{\mathrm{a} \rightarrow \mathrm{b}}\right\}, \max _{\mathrm{a}, \mathrm{b} \in \Sigma}\left\{\gamma_{\mathrm{a} \rightarrow \mathrm{b}}\right\}\right\} .
$$

Então, $(c, k)$ é A-ótimo para $g$.

Prova. Seja $\left(c^{\prime}, k^{\prime}\right)$ correspondente a um alinhamento arbitrário $A^{\prime}$. Note que $g>0$. Para provar a proposição, desde que $k$ é mínimo, vamos mostrar que $(i)$ se $k=k^{\prime}$, então $c+g k \leq$ $c^{\prime}+g k^{\prime}$; e $(i i)$ se $k<k^{\prime}$, então $c+g k<c^{\prime}+g k^{\prime}$.

Suponha que $k=k^{\prime}$. Segue do Fato 16 que $|A|=\left|A^{\prime}\right|$, o que implica, sendo $A$ de menor pontuação entre os alinhamentos de comprimento mínimo, que $c+g k \leq c^{\prime}+g k^{\prime}$ e $(i)$ está provado.

Suponha então que $k<k^{\prime}$. Segue do Fato 16 que $|A|<\left|A^{\prime}\right|$ e $k \leq k^{\prime}-2$. Analisamos os dois casos possíveis: o caso $\max _{\mathrm{a}, \mathrm{b} \in \Sigma}\left\{\gamma_{\mathrm{a} \rightarrow \mathrm{b}}\right\} \geq 0$ e o caso $\max _{\mathrm{a}, \mathrm{b} \in \Sigma}\left\{\gamma_{\mathrm{a} \rightarrow \mathrm{b}}\right\}<0$.

Suponha que $\max _{\mathrm{a}, \mathrm{b} \in \Sigma}\left\{\gamma_{\mathrm{a} \rightarrow \mathrm{b}}\right\} \geq 0$. Logo,

$$
\begin{aligned}
c+g k & \leq N\left(\max _{\mathrm{a}, \mathrm{b} \in \Sigma}\left\{\gamma_{\mathrm{a} \rightarrow \mathrm{b}}\right\}\right)+g\left(k^{\prime}-2\right) \\
& <g+g\left(k^{\prime}-2\right)=-g+g k^{\prime} \\
& <\frac{N-k^{\prime}}{2} \min _{\mathrm{a}, \mathrm{b} \in \Sigma}\left\{\gamma_{\mathrm{a} \rightarrow \mathrm{b}}\right\}+g k^{\prime} \\
& \leq c^{\prime}+g k^{\prime} .
\end{aligned}
$$

onde (2.6) vale pois, sendo $\max _{\mathrm{a}, \mathrm{b} \in \Sigma}\left\{\gamma_{\mathrm{a} \rightarrow \mathrm{b}}\right\} \geq 0$ e a quantidade de substituições não superior a $N$, vale que $c \leq N \max _{\mathrm{a}, \mathrm{b} \in \Sigma}\left\{\gamma_{\mathrm{a} \rightarrow \mathrm{b}}\right\}$, e sendo $k \leq k^{\prime}-2$ e $g>0$, vale que $g k \leq g\left(k^{\prime}-2\right) ;(2.7)$ vale pela hipótese da proposição; (2.8) vale pelo Fato 20, e (2.9) segue de (2.5). Portanto, se $k<k^{\prime}$ e $\max _{\mathrm{a}, \mathrm{b} \in \Sigma}\left\{\gamma_{\mathrm{a} \rightarrow \mathrm{b}}\right\} \geq 0$, então $c+g k<c^{\prime}+g k^{\prime}$, o que implica que nesse caso vale (ii). 
Suponha $\max _{a, b \in \Sigma}\left\{\gamma_{a \rightarrow b}\right\}<0$. Segue que $\min _{a, b \in \Sigma}\left\{\gamma_{a \rightarrow b}\right\}<0$. Logo,

$$
\begin{aligned}
c+g k & \leq g k \\
& \leq g\left(k^{\prime}-2\right) \\
& \leq-g+g k^{\prime} \\
& <\frac{N-k^{\prime}}{2} \min _{\mathrm{a}, \mathrm{b} \in \Sigma}\left\{\gamma_{\mathrm{a} \rightarrow \mathrm{b}}\right\}+g k^{\prime}, \\
& \leq c^{\prime}+g k^{\prime} .
\end{aligned}
$$

onde (2.10) segue de $\max _{\mathrm{a}, \mathrm{b} \in \Sigma}\left\{\gamma_{\mathrm{a} \rightarrow \mathrm{b}}\right\}<0 ;$ (2.11) segue de $g>0$ e $k \leq k^{\prime}-2$; (2.12) segue de $g>0 ;(2.13)$ segue do Fato 20 e; (2.14) segue de (2.5). Portanto, vale (ii) também quando $\max _{\mathrm{a}, \mathrm{b} \in \Sigma}\left\{\gamma_{\mathrm{a} \rightarrow \mathrm{b}}\right\}<0$ e a prova termina.

Proposição 22. Sejam

$$
e=\frac{\min _{\mathrm{a}, \mathrm{b} \in \Sigma}\left\{\gamma_{\mathrm{a} \rightarrow \mathrm{b}}\right\}}{2} \quad \text { e } \quad f=N \cdot \max \left\{-\min _{\mathrm{a}, \mathrm{b} \in \Sigma}\left\{\gamma_{\mathrm{a} \rightarrow \mathrm{b}}\right\}, \max _{\mathrm{a}, \mathrm{b} \in \Sigma}\left\{\gamma_{\mathrm{a} \rightarrow \mathrm{b}}\right\}\right\}+\varepsilon,
$$

para algum número $\varepsilon>0$. Então, $\mathcal{H}=\mathcal{H}[e, f]$.

Prova. Seja $\mathcal{H}=\left\{\left(c_{0}, k_{0}\right), \ldots,\left(c_{\mathcal{N}}, k_{\mathcal{N}}\right)\right\}$ tal que $\left(c_{i}, k_{i}\right) \neq\left(c_{i+1}, k_{i+1}\right)$ para cada $0 \leq i<\mathcal{N}$ e $k_{0} \geq \ldots \geq k_{\mathcal{N}}$. Segue do Fato 17 que $k_{i} \neq k_{i+1}$ para cada $i$, o que implica que $k_{0}>\ldots>k_{\mathcal{N}}$.

Pela Proposição $19,\left(c_{0}, k_{0}\right)$ é A-ótimo para e e, pela Proposição $21,\left(c_{\mathcal{N}}, k_{\mathcal{N}}\right)$ é A-ótimo para $f$. Logo $\left(c_{0}, k_{0}\right),\left(c_{\mathcal{N}}, k_{\mathcal{N}}\right) \in \mathcal{H}[e, f]$.

Seja $(c, k) \in \mathcal{H}$ um elemento arbitrário, $(c, k) \neq\left(c_{0}, k_{0}\right),(c, k) \neq\left(c_{\mathcal{N}}, k_{\mathcal{N}}\right)$. Segue que $k_{0}>k>k_{\mathcal{N}}$. Como $(c, k) \in \mathcal{H}$, existe $g$ tal que $(c, k)$ é A-ótimo para $g$. Sendo $k_{0}>k$ e $k>k_{\mathcal{N}}$, segue do Fato 18 que $e<g$ e $g<f$. Logo $(c, k) \in \mathcal{H}[e, f]$. Como o argumento vale para cada $(c, k) \in \mathcal{H}$ exceto $\left(c_{0}, k_{0}\right)$ e $\left(c_{\mathcal{N}}, k_{\mathcal{N}}\right)$, e $\left(c_{0}, k_{0}\right),\left(c_{\mathcal{N}}, k_{\mathcal{N}}\right) \in \mathcal{H}[e, f]$, segue que $\mathcal{H} \subseteq \mathcal{H}[e, f]$. Segue de $(2.4)$ que $\mathcal{H}=\mathcal{H}[e, f]$.

Para um alinhamento $A$, sendo $|A|=(N+k(A)) / 2$, a pontuação- $v \mathrm{~N}_{\gamma}$ de $A$ é

$$
v \mathrm{~N}_{\gamma}(A)=\frac{c_{\gamma}(A)+g k(A)}{(N+k(A)) / 2}=2 \frac{c_{\gamma}(A)+g k(A)}{N+k(A)} .
$$

Dizemos que o custo normalizado do par $(c, k)$ para $g$ é $2(c+g k) /(N+k)$. Dizemos que o $\operatorname{par}(c, k) \in \mathbb{A}$ é N-ótimo para $g$ se, para cada $\left(c^{\prime}, k^{\prime}\right) \in \mathbb{A}$, temos que

$$
\frac{c+g k}{N+k} \leq \frac{c^{\prime}+g k^{\prime}}{N+k^{\prime}}
$$

O Algoritmo 3 abaixo usa a estrutura estudada para calcular opt $\mathrm{N}_{\gamma}(s, t)$. Consideramos, para cada $\mathrm{a}, \mathrm{b} \in \Sigma$, que $\gamma_{\mathrm{a} \rightarrow \mathrm{b}}$ é um número inteiro.

Vamos argumentar que o algoritmo está correto. O Lema 24 mostra que, no início de cada iteração na Linha 5 , um elemento em $\mathcal{H}[e, f]$, digamos $\left(c_{g}, k_{g}\right)$, é N-ótimo para $g$. Suponha $f-e<1 / N^{2}$, ou seja, o início da última iteração. A contrapositiva do Lema 27 garante existir no máximo dois elementos em $\mathcal{H}[e, f]$. Logo, um deles deve ser $\left(c_{g}, k_{g}\right)$. Consideramos o caso de $|\mathcal{H}[e, f]|=1$ e o caso de $|\mathcal{H}[e, f]|=2$. Se $|\mathcal{H}[e, f]|=1$, então $\left(c_{g}, k_{g}\right)$ é A-ótimo para e e é A-ótimo para $f$ e o algoritmo devolve corretamente o custo normalizado de $\left(c_{g}, k_{g}\right)$. Se $|\mathcal{H}[e, f]|=2$, o Lema 28 garante que os elementos A-ótimos para $e$ e para $f$, digamos $\left(c_{e}, k_{e}\right)$ e $\left(c_{f}, k_{f}\right)$, são diferentes. Segue que $\left(c_{g}, k_{g}\right)=\left(c_{e}, k_{e}\right)$ ou 


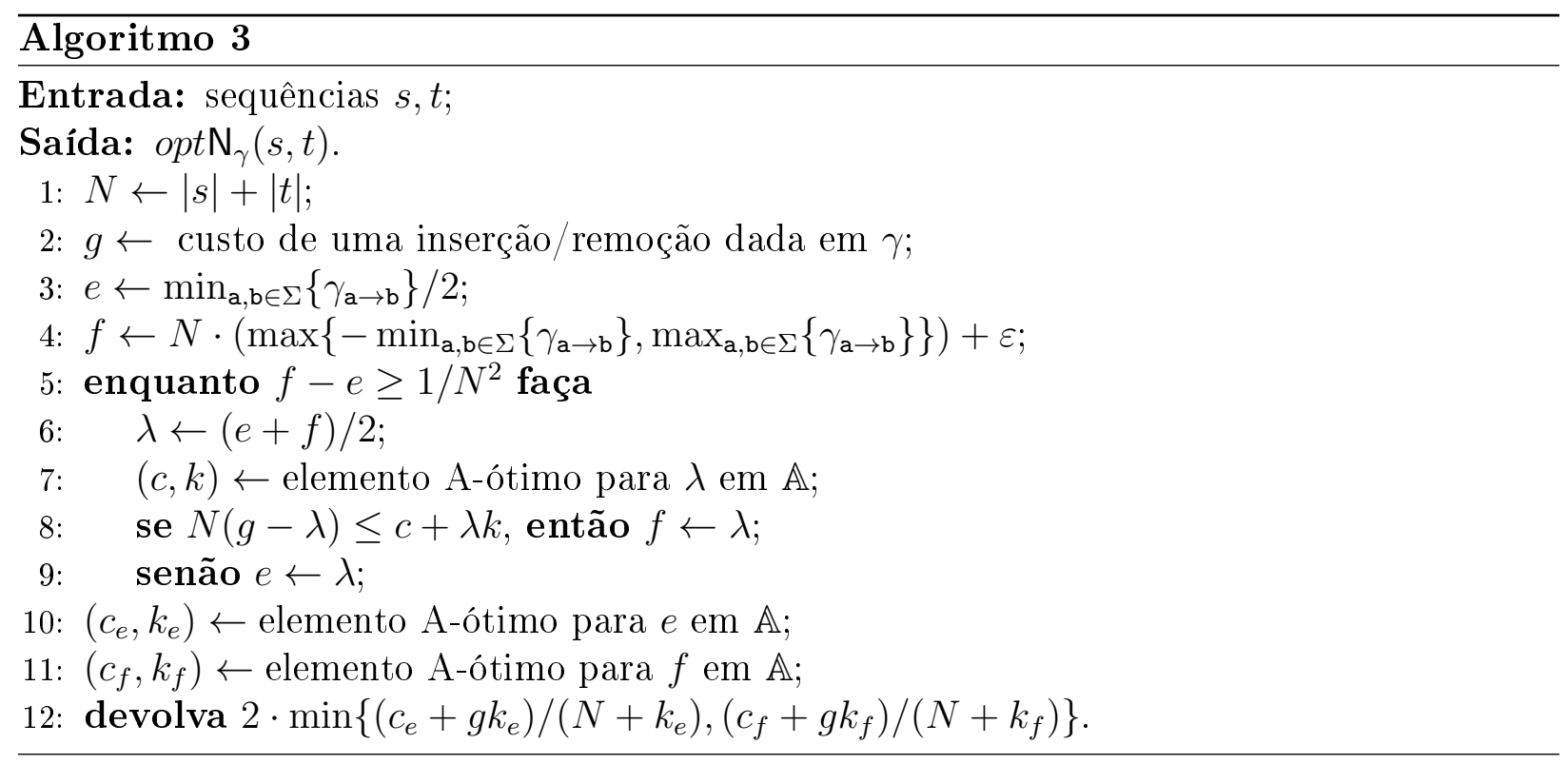

$\left(c_{g}, k_{g}\right)=\left(c_{f}, k_{f}\right)$. Desde que $\left(c_{g}, k_{g}\right)$ tem custo normalizado mínimo, segue que, também nesse caso, o algoritmo devolve corretamente o valor normalizado de $\left(c_{g}, k_{g}\right)$.

O seguinte resultado é usado para a prova do funcionamento do algoritmo.

Fato 23. Sejam $(c, k),\left(c_{g}, k_{g}\right) \in \mathbb{A}$ e $g, \lambda$ números reais tais que

$$
\begin{aligned}
c+\lambda k & \leq c_{g}+\lambda k_{g}, \\
\frac{c_{g}+g k_{g}}{N+k_{g}} & <\frac{c+g k}{N+k} .
\end{aligned}
$$

Então, se $k_{g}>k$, então $N(g-\lambda)<c+\lambda k$; e se $k>k_{g}$, então $N(g-\lambda)>c+\lambda k$.

Prova. Como $N>0$ e $k_{g}>0$, temos, usando (2.15) na primeira e (2.16) na segunda desigualdade abaixo, que

$$
\frac{c+\lambda k+(g-\lambda) k_{g}}{N+k_{g}} \leq \frac{c_{g}+\lambda k_{g}+(g-\lambda) k_{g}}{N+k_{g}}=\frac{c_{g}+g k_{g}}{N+k_{g}}<\frac{c+g k}{N+k} .
$$

Logo, $\left(c+\lambda k+(g-\lambda) k_{g}\right) /\left(N+k_{g}\right)<(c+g k) /(N+k)$. Segue, sendo $N>0, k, k_{g} \geq 0$, que

$$
N(g-\lambda)\left(k_{g}-k\right)<(c+\lambda k)\left(k_{g}-k\right) .
$$

Portanto, se $k_{g}>k$, então segue que $N(g-\lambda)<c+\lambda k$; e se $k>k_{g}$, então segue que $N(g-\lambda)>c+\lambda k$.

Lema 24. Cada vez que a Linha 5 do Algoritmo 3 é executada, para algum $\left(c_{g}, k_{g}\right)$ N-ótimo para $g$, vale que $\left(c_{g}, k_{g}\right) \in \mathcal{H}[e, f]$.

Prova. A prova é por indução no número de iterações. A Proposição 22 e os valores atribuídos a $e$ e $f$ nas Linhas 3 e 4 garantem que no início da primeira iteração $\mathcal{H}[e, f]=\mathcal{H}$. Segue do Fato 26 que o lema vale no início da primeira iteração.

Suponha que estamos no início de uma iteração qualquer que não seja a última. A hipótese de indução garante existir $\left(c_{g}, k_{g}\right)$ que é N-ótimo para $g$ e que é A-ótimo para algum $\bar{g}$, onde $e \leq \bar{g} \leq f$. Seja $(c, k)$ A-ótimo para $\lambda=(e+f) / 2$ definido ao executar a 
Linha 7. Consideramos dois casos: $(c, k)$ é N-ótimo e $(c, k)$ não é N-ótimo para $g$. Se $(c, k)$ é N-ótimo para $g$, desde que $(c, k) \in \mathcal{H}[e, \lambda]$ e $(c, k) \in \mathcal{H}[\lambda, f]$, temos que a afirmação vale trivialmente para a próxima iteração e o passo de indução está concluído e o lema provado. Assumimos, então que $(c, k)$ não é N-ótimo para $g$.

Como, $\left(c_{g}, k_{g}\right)$ é N-ótimo e $(c, k)$ não é N-ótimo para $g$, temos que

$$
\frac{c_{g}+g k_{g}}{N+k_{g}}<\frac{c+g k}{N+k}
$$

e que $\left(c_{g}, k_{g}\right) \neq(c, k)$. Como $(c, k)$ e $\left(c_{g}, k_{g}\right)$ são A-ótimos e $\left(c_{g}, k_{g}\right) \neq(c, k)$, temos pela contrapositiva do Fato 17 que $k_{g} \neq k$. Logo, $k_{g}>k$ ou $k>k_{g}$.

Suponha $k_{g}>k$. Como $\left(c_{g}, k_{g}\right)$ e $(c, k)$ são A-ótimos para $\bar{g}$ e $\lambda$ respectivamente, temos pelo Fato 18 que $\bar{g} \leq \lambda$, o que implica, sendo $e \leq \bar{g}$, que $e \leq \bar{g} \leq \lambda$ o que, por sua vez, implica, sendo $\left(c_{g}, k_{g}\right)$ A-ótimo para $\bar{g}$, que $\left(c_{g}, k_{g}\right) \in \mathcal{H}[e, \lambda]$. Por outro lado, como $k_{g}>k$, $c+\lambda k \leq c_{g}+\lambda k_{g}$ e vale (2.17), pelo Fato 23 temos que $N(g-\lambda)<c+\lambda k$ e, portanto, de acordo com a execução da Linha 8 do Algoritmo 3, o conjunto considerado na próxima iteração é $\mathcal{H}[e, \lambda]$ que contém o elemento N-ótimo $\left(c_{g}, k_{g}\right)$. Logo, vale a afirmação no início da próxima iteração.

Se $k>k_{g}$, então, usando resultados e raciocínio similares, temos que, de acordo com a execução do Algoritmo 3, a afirmação também vale no início da próxima iteração.

Fato 25. Sejam $\left(c_{1}, k_{1}\right),\left(c_{2}, k_{2}\right) \in \mathcal{H}$ com $k_{1}>k_{2}$, tais que, para cada $(c, k) \in \mathcal{H}, k \geq k_{1}$ ou $k \leq k_{2}$. Então, $\left(c_{1}, k_{1}\right)$ é A-ótimo para $\bar{g}=\left(c_{2}-c_{1}\right) /\left(k_{1}-k_{2}\right)$.

Prova. Como $\bar{g}=\left(c_{2}-c_{1}\right) /\left(k_{1}-k_{2}\right)$, temos que $c_{1}+\bar{g} k_{1}=c_{2}+\bar{g} k_{2}$.

Sejam $g_{1}, g_{2}$ números tais que $\left(c_{1}, k_{1}\right)$ e $\left(c_{2}, k_{2}\right)$ são A-ótimos para $g_{1}$ e $g_{2}$, respectivamente. Segue do Fato 18 que $g_{1} \leq \bar{g}=\left(c_{2}-c_{1}\right) /\left(k_{1}-k_{2}\right)<g_{2}$.

Seja também $(c, k) \in \mathcal{H}$ arbitrário tal que $(c, k) \neq\left(c_{1}, k_{1}\right)$ e $(c, k) \neq\left(c_{2}, k_{2}\right)$. Como, por hipótese, $k \geq k_{1}$ ou $k \leq k_{2}$, segue do Fato 17 que $k>k_{1}$ ou $k<k_{2}$.

Suponha que $k>k_{1}$. Pela definição de $g_{1}$, temos que $c_{1}+g_{1} k_{1}<c+g_{1} k$, o que implica, sendo $k>k_{1}$, que $\left(c_{1}-c\right) /\left(k-k_{1}\right)<g_{1}$ o que, por sua vez, implica, sendo $g_{1} \leq \bar{g}$, que $\left(c_{1}-c\right) /\left(k-k_{1}\right)<\bar{g}$. Logo, usando novamente o fato de $k>k_{1}$, segue que $c_{1}+\bar{g} k_{1}<c+\bar{g} k$. Portanto, se $k>k_{1}$, então $c_{1}+\bar{g} k_{1}<c+\bar{g} k$.

Suponha que $k_{2}>k$. Pela definição de $g_{2}$, temos que $c_{2}+g_{2} k_{2} \leq c+g_{2} k$, o que implica, sendo $k_{2}>k$, que $g_{2} \leq\left(c-c_{2}\right) /\left(k_{2}-k\right)$, o que, por sua vez, implica, sendo $\bar{g}<g_{2}$, que $\bar{g}<\left(c-c_{2}\right) /\left(k_{2}-k\right)$. Logo, usando novamente o fato de $k_{2}>k$, segue que $c_{2}+\bar{g} k_{2}<c+\bar{g} k$. Desde que $c_{1}+\bar{g} k_{1}=c_{2}+\bar{g} k_{2}$, segue que $c_{1}+\bar{g} k_{1}=c_{2}+\bar{g} k_{2}<c+\bar{g} k$. Portanto, se $k_{2}>k$, então $c_{1}+\bar{g} k_{1}<c+\bar{g} k$.

Como, independentemente do valor de $k, c_{1}+\bar{g} k_{1}<c+\bar{g} k$, sendo $(c, k) \in \mathcal{H}$ arbitrário, temos que $\left(c_{1}, k_{1}\right)$ é A-ótimo para $\bar{g}=\left(c_{2}-c_{1}\right) /\left(k_{1}-k_{2}\right)$.

Fato 26. Seja $(c, k)$ N-ótimo para $g \in \mathbb{R}$. Logo, $(c, k) \in \mathcal{H}$.

Prova. Seja $\mathcal{H}=\left\{\left(c_{0}, k_{0}\right), \ldots,\left(c_{\mathcal{N}}, k_{\mathcal{N}}\right)\right\}$ tal que $\left(c_{i}, k_{i}\right) \neq\left(c_{i+1}, k_{i+1}\right)$ para cada $0 \leq i<\mathcal{N}$ e $k_{0} \geq \ldots \geq k_{\mathcal{N}}$. Pela Proposição $19,\left(c_{0}, k_{0}\right)$ corresponde a um alinhamento de comprimento máximo e pela Proposição 21, $\left(c_{\mathcal{N}}, k_{\mathcal{N}}\right)$ corresponde a um alinhamento de comprimento mínimo. Segue do Fato 16 que $k_{\mathcal{N}} \leq k \leq k_{0}$. Seja $i$ o inteiro tal que $k_{i} \geq k \geq k_{i+1}$ que o argumento $k_{0} \geq k \geq k_{\mathcal{N}}$ garante existir. 
Segue do Fato 25 que $\left(c_{i}, k_{i}\right)$ é A-ótimo para $\bar{g}=\left(c_{i+1}-c_{i}\right) /\left(k_{i}-k_{i+1}\right)$, o que implica que $c_{i}+\bar{g} k_{i} \leq c+\bar{g} k$. Sendo $c_{i+1}+\bar{g} k_{i+1}=c_{i}+\bar{g} k_{i}$, segue que,

$$
c_{i+1}+\bar{g} k_{i+1}=c_{i}+\bar{g} k_{i} \leq c+\bar{g} k \text {. }
$$

Além disso, como $(c, k)$ é N-ótimo para $g$,

$$
\frac{c+g k}{N+k} \leq \min \left\{\frac{c_{i}+g k_{i}}{N+k_{i}}, \frac{c_{i+1}+g k_{i+1}}{N+k_{i+1}}\right\} .
$$

Se $k<k_{i}$ e $k>k_{i+1}$, então desde que vale (2.18) e (2.19), temos, pelo Fato 23, que $N(g-\bar{g})>c_{i}+\bar{g} k_{i}$ e que $N(g-\bar{g})<c_{i+1}+\bar{g} k_{i+1}$, o que implica $c_{i}+\bar{g} k_{i}<c_{i+1}+\bar{g} k_{i+1}$, uma contradição com (2.18). Logo, $k \nless k_{i}$ ou $k \ngtr k_{i+1}$, o que implica, sendo $k_{i} \geq k \geq k_{i+1}$ que $k=k_{i}$ ou $k=k_{i+1}$.

Se $k_{i}=k$, então, como consequência de (2.18), temos que $c_{i} \leq c$ e, como consequência de (2.19), temos que $c \leq c_{i}$, o que implica que $c_{i}=c$. Segue que $(c, k)=\left(c_{i}, k_{i}\right)$ e a prova termina para esse caso. Usando raciocínio análogo, se $k_{i+1}=k$, então $(c, k)=\left(c_{i}, k_{i+1}\right)$ e a prova também termina.

Lema 27. Se $|\mathcal{H}[e, f]| \geq 3$, então $f-e \geq 1 / N^{2}$.

Prova. Suponha que $|\mathcal{H}[e, f]| \geq 3$. Então, existem 3 números reais $g_{1}, g_{2}, g_{3}$ e 3 elementos 2-a-2 distintos $\left(c_{1}, k_{1}\right),\left(c_{2}, k_{2}\right),\left(c_{3}, k_{3}\right)$, tais que $e \leq g_{1}, g_{2}, g_{3} \leq f, k_{1} \geq k_{2} \geq k_{3}$ e $\left(c_{1}, k_{1}\right)$ é A-ótimo para $g_{1},\left(c_{2}, k_{2}\right)$ é A-ótimo para $g_{2}$ e $\left(c_{3}, k_{3}\right)$ é A-ótimo para $g_{3}$.

Como $\left(c_{1}, k_{1}\right),\left(c_{2}, k_{2}\right),\left(c_{3}, k_{3}\right)$ são 2 -a-2 distintos, temos que a contrapositiva do Fato 17 garante que $k_{1}, k_{2}, k_{3}$ são 2 -a-2 distintos. Segue daí que $k_{1}>k_{2}>k_{3}$. Segue do Fato 18 que

$$
g_{1} \leq \frac{c_{2}-c_{1}}{k_{1}-k_{2}}<g_{2} \leq \frac{c_{3}-c_{2}}{k_{2}-k_{3}}<g_{3}
$$

Logo,

$$
\begin{aligned}
g_{3}-g_{1} & \geq \frac{c_{3}-c_{2}}{k_{2}-k_{3}}-\frac{c_{2}-c_{1}}{k_{1}-k_{2}} \\
& =\frac{c_{1}\left(k_{2}-k_{3}\right)+c_{3}\left(k_{1}-k_{2}\right)-c_{2}\left(k_{1}-k_{3}\right)}{\left(k_{2}-k_{3}\right)\left(k_{1}-k_{2}\right)} \\
& \geq \frac{c_{1}\left(k_{2}-k_{3}\right)+c_{3}\left(k_{1}-k_{2}\right)-c_{2}\left(k_{1}-k_{3}\right)}{N^{2}} \\
& \geq \frac{1}{N^{2}},
\end{aligned}
$$

onde a segunda desigualdade segue de $N \geq k_{1}-k_{2}>0$ e $N \geq k_{2}-k_{3}>0$ pois $N \geq k_{1}>$ $k_{2}>k_{3}>0$; e a última desigualdade segue de $c_{1}, c_{2}, c_{3}, k_{1}, k_{2}, k_{3}$ serem números inteiros e $c_{1}\left(k_{2}-k_{3}\right)+c_{3}\left(k_{1}-k_{2}\right)-c_{2}\left(k_{1}-k_{3}\right)>0$, pois $\left(c_{2}-c_{1}\right) /\left(k_{1}-k_{2}\right)<\left(c_{3}-c_{2}\right)\left(k_{2}-k_{3}\right)$.

Portanto, sendo $e \leq g_{1}, g_{3} \leq f$ e $g_{3}-g_{1} \geq 1 / N^{2}$ segue que $f-e \geq g_{3}-g_{1} \geq 1 / N^{2}$.

Lema 28. Se $|\mathcal{H}[e, f]| \geq 2,\left(c_{e}, k_{e}\right)$ é A-ótimo para $e$ e $\left(c_{f}, k_{f}\right)$ é A-ótimo para $f$, então $\left(c_{e}, k_{e}\right) \neq\left(c_{f}, k_{f}\right)$.

Prova. Suponha que $|\mathcal{H}[e, f]| \geq 2,\left(c_{e}, k_{e}\right)$ e $\left(c_{f}, k_{f}\right)$ A-ótimos para $e$ e $f$ respectivamente. Como $|\mathcal{H}[e, f]| \geq 2$, existem $(c, k)$ e $g$ tais que $(c, k) \neq\left(c_{e}, k_{e}\right),(c, k)$ é A-ótimo para $g$ e $e \leq g \leq f$. 
Como $\left(c_{e}, k_{e}\right),(c, k)$ e $\left(c_{f}, k_{f}\right)$ são A-ótimos para $e, g$ e $f$ respectivamente e $e \leq g \leq f$, temos como consequência da contrapositiva do Fato 18 que $k_{e} \geq k \geq k_{f}$.

Como $\left(c_{e}, k_{e}\right)$ e $(c, k)$ são A-ótimos e $(c, k) \neq\left(c_{e}, k_{e}\right)$, temos, pela contrapositiva do Fato 17 , que $k_{e} \neq k$. Segue que $k_{e}>k \geq k_{e}$. Logo, $k_{e}>k_{f}$ e, portanto, $\left(c_{e}, k_{e}\right) \neq\left(c_{f}, k_{f}\right)$.

A seguir estudamos o tempo gasto pelo algoritmo.

Teorema 29. Para entrada $s, t \in \Sigma^{*}$, com $|s|=|t|=n$, e considerando custo uniforme para inserção e remoção, o Algoritmo 3 gasta tempo $O\left(T_{\mathrm{APS}}(n) \cdot \log n\right)$.

Prova. Inicialmente, $f-e$ não é superior a $4 n \cdot \max \left\{\left|\min _{\mathrm{a}, \mathrm{b} \in \Sigma} \gamma_{\mathrm{a} \rightarrow \mathrm{b}}\right|,\left|\max _{\mathrm{a}, \mathrm{b} \in \Sigma} \gamma_{\mathrm{a} \rightarrow \mathrm{b}}\right|\right\}$. Em cada iteração esse valor é dividido por 2 . Em

$$
O\left(\log \left(n^{2} \cdot 4 n \cdot \max \left\{\left|\min _{\mathrm{a}, \mathrm{b} \in \Sigma} \gamma_{\mathrm{a} \rightarrow \mathrm{b}}\right|,\left|\max _{\mathrm{a}, \mathrm{b} \in \Sigma} \gamma_{\mathrm{a} \rightarrow \mathrm{b}}\right|\right\}\right)\right)
$$

iterações esse valor é menor que $1 / n^{2}$. Se consideramos constantes os valores de $\gamma$, então o número de iterações é $O\left(\log 4 n^{3}\right)=O(\log n)$. Em cada iteração, o algoritmo gasta tempo $T_{\mathrm{APS}}(n)$ para determinar um alinhamento A-ótimo de $(s, t)$. Portanto, o tempo total gasto é $O\left(T_{\mathrm{APS}}(n) \cdot \log n\right)$.

A Tabela 2.1 resume os resultados descritos nesta seção para o alinhamento de duas sequências.

\begin{tabular}{|c|c|c|c|}
\hline Problema & Restrição* & Tempo** & Referência \\
\hline \multirow{3}{*}{ APS } & sem restrições & $O\left(n^{2}\right)$ & [NW70] \\
\hline & $\gamma_{\mathrm{a} \rightarrow \mathrm{b}}= \begin{cases}0 & \text { se } \mathrm{a}=\mathrm{b} \\
1 & \text { caso contrário }\end{cases}$ & $O\left(n^{2} / \log n\right)$ & [MP80] \\
\hline & $\gamma$ com entradas racionais & $O\left(n^{2} / \log n\right)$ & [CLZU02] \\
\hline \multirow{5}{*}{ APSN } & 2-aproximação sem restrição & $O\left(n^{2}\right)$ & descrita nesta tese \\
\hline & sem restrições & $O\left(n^{3}\right)$ & [MV93] \\
\hline & sem restrições & $\begin{array}{l}O\left(T_{\mathrm{APS}}(n) \cdot n\right) \\
\text { mas rápido na prática }\end{array}$ & [VMA95] \\
\hline & $\gamma$ com entradas racionais & $O\left(T_{\mathrm{APS}}(n) \cdot \log n\right)$ & [AE00] \\
\hline & $\begin{array}{l}\gamma \text { com custo uniforme } \\
\text { nas operações de edição }\end{array}$ & $O\left(T_{\mathrm{APS}}(n) \cdot \log n\right)$ & descrita nesta tese \\
\hline
\end{tabular}

Tabela 2.1: (*) é a restrição da matriz de pontuação $\gamma$; (**) é o tempo assintótico gasto para comparar duas sequências de comprimento $n$ cada.

\subsection{Alinhamento de várias sequências}

Considere uma matriz de pontuação $\gamma$ fixa. Sejam $S=\left(s_{1}, \ldots, s_{k}\right) \in\left(\Sigma^{*}\right)^{k}$ e um alinhamento $A=\left[s_{1}^{\prime}, \ldots, s_{k}^{\prime}\right]$ de $S$. O critério $v \mathrm{SP}_{\gamma}$, também chamado pontuação-SP, para pontuar o alinhamento $A$ é definido como

$$
v \mathrm{SP}_{\gamma}(A)=\sum_{h=1}^{k-1} \sum_{i=h+1}^{k} v \mathrm{~A}_{\gamma}\left(A_{\{h, i\}}\right) .
$$


Definimos opt $\mathrm{SP}_{\gamma}$ como a função ótima para o critério $v \mathrm{SP}_{\gamma}$. Um alinhamento $A$ de $S$ tal que $v \mathrm{SP}_{\gamma}(A)=\operatorname{optSP}_{\gamma}(S)$ é dito alinhamento $v \mathrm{SP}_{\gamma}$-ótimo. O seguinte problema está associado ao critério $v \mathrm{SP}_{\gamma}$.

Problema 3. AVS: Alinhamento de várias sequências

Dado $S \in\left(\Sigma^{*}\right)^{k}$, determinar optSP $\gamma(S)$, onde $\gamma$ é uma matriz de pontuação fixa.

\subsubsection{Algoritmos exatos}

Mostramos a seguir um algoritmo baseado em programação dinâmica para o problema AVS. Esse algoritmo é uma generalização do algoritmo básico utilizado para calcular a pontuação do alinhamento de duas sequências. Uma tabela $D$ armazena os valores ótimos dos sub-problemas calculadas pela programação dinâmica. A tabela possui $k$ dimensões e cada $D\left(j_{1}, j_{2}, \ldots, j_{k}\right)$ armazena o valor de $\operatorname{optSP}_{\gamma}\left(\left(s_{1}\left(1 \ldots j_{1}\right), \ldots, s_{k}\left(1 \ldots j_{k}\right)\right)\right)$.

A pontuação-SP de uma k-upla $\left[x_{1}, \ldots, x_{k}\right]$, onde cada $x_{i} \in \Sigma$, é definida como

$$
v \mathrm{SP}_{\gamma}\left(\left[x_{1}, \ldots, x_{k}\right]\right)=\sum_{h=1}^{k-1} \sum_{i=h+1}^{k} \gamma_{x_{h} \rightarrow x_{i}} .
$$

Assim, se $c_{j}$ é a coluna $j$ de um alinhamento $A=\left[s_{1}^{\prime}, \ldots, s_{k}^{\prime}\right]$, então a pontuação-SP de $A$ pode ser calculada

$$
v \mathrm{SP}_{\gamma}(A)=\sum_{j=1}^{|A|} v \mathrm{SP}_{\gamma}\left(c_{j}\right) .
$$

Dado $S=\left(s_{1}, \ldots, s_{k}\right)$ onde cada $s_{i}$ possui $n_{i}$ símbolos, definimos

$$
\vec{V}_{S}=\left\{0, \ldots, n_{1}\right\} \times\left\{0, \ldots, n_{2}\right\} \times \ldots \times\left\{0, \ldots, n_{k}\right\} .
$$

A tabela $D$ é indexada por $\vec{V}_{S}$. Um elemento de $\vec{j}=\left(j_{1}, \ldots, j_{k}\right) \in \vec{V}_{S}$ é denotado $\vec{j}=$ $\left[j_{1}, \ldots, j_{k}\right]$. Definimos $S(\vec{j})=\left(s_{1}\left(1 \ldots j_{1}\right), \ldots, s_{k}\left(1 \ldots j_{k}\right)\right)$. A entrada $D(\vec{j})$ armazena portanto optSP $(S(\vec{j}))$. Seja $\mathcal{B}^{k}=\{0,1\}^{k} \backslash \mathbf{0}^{k}$, onde $\mathbf{0}^{k}=[0, \ldots, 0]$ denota uma sequência de $k$ zeros. Para $\vec{j}=\left[j_{1}, \ldots, j_{k}\right] \in \vec{V}_{S}$ e $\vec{b}=\left[b_{1}, \ldots, b_{k}\right] \in \mathcal{B}^{k}$, definimos

$$
\vec{b} \cdot S(\vec{j})=\left[x_{1}, \ldots, x_{k}\right] \in\left(\Sigma_{\sqcup}\right)^{k}
$$

tal que

$$
x_{i}= \begin{cases}s_{i}\left(j_{i}\right) & \text { se } b_{i}=1 \\ \iota & \text { caso contrário. }\end{cases}
$$

Assim, dado um alinhamento $A$ de $S(\vec{j})$, existe $\vec{b} \in \mathcal{B}^{k}$ tal que $A(|A|)=\vec{b} \cdot S(\vec{j})$. Neste caso, sendo $\vec{j}=\left[j_{1}, \ldots, j_{k}\right]$ e $\vec{b}=\left[b_{1}, \ldots, b_{k}\right]$, temos que $b_{i}=1$ se e somente se $s_{i}\left(j_{i}\right)$ está na $i$-ésima linha da última coluna de $A$.

Definimos também a operação

$$
\vec{j}-\vec{b}=\left[j_{1}-b_{1}, \ldots, j_{k}-b_{k}\right] .
$$

Note que, se não existe $i$ tal que $j_{i}=0$ e $b_{i}=1$, ou seja, se $j_{i} \geq b_{i}$ para cada $i$, então $S(\vec{j}-\vec{b})$ é a $k$-upla $S(\vec{j})$ removendo os símbolos que estão na última coluna de um alinhamento onde $\vec{b} \cdot S(\vec{j})$ é a última coluna. Se $\vec{j}=\left[j_{1}, \ldots, j_{k}\right]$ e $\vec{b}=\left[b_{1}, \ldots, b_{k}\right]$, então denotamos $\vec{b} \leq \vec{j}$ se $j_{i}-b_{i} \geq 0$ para cada $i$. 
Então, $D(\vec{j})=\operatorname{optSP} \gamma(S(\vec{j}))$ é o menor valor

$$
\operatorname{optSP} \gamma(S(\vec{j}-\vec{b}))+v \mathrm{SP}_{\gamma}(\vec{b} \cdot S(\vec{j}))
$$

quando consideramos todas as possibilidades para $\vec{b} \leq \vec{j}$. Portanto, $D\left(\mathbf{0}^{k}\right)=0$ e cada entrada da tabela de programação dinâmica $D(\vec{j}) \operatorname{com} \vec{j} \neq \mathbf{0}^{k}$ é calculada por

$$
D(\vec{j})=\min _{\vec{b} \in \mathcal{B}^{k}, \vec{b} \leq \vec{j}}\left\{D(\vec{j}-\vec{b})+v \mathrm{SP}_{\gamma}(\vec{b} \cdot S(\vec{j}))\right\} .
$$

Quando calculamos o valor de $D(\vec{j})$, devemos garantir que $D(\vec{j}-\vec{b})$ já tenha sido calculado para todos os valores de $\vec{b}$. Isso pode ser feito gerando os índices de $D$ em ordem lexicográfica usando um algoritmo que simula o hodômetro de um automóvel [Bri03].

A seguir apresentamos o algoritmo.

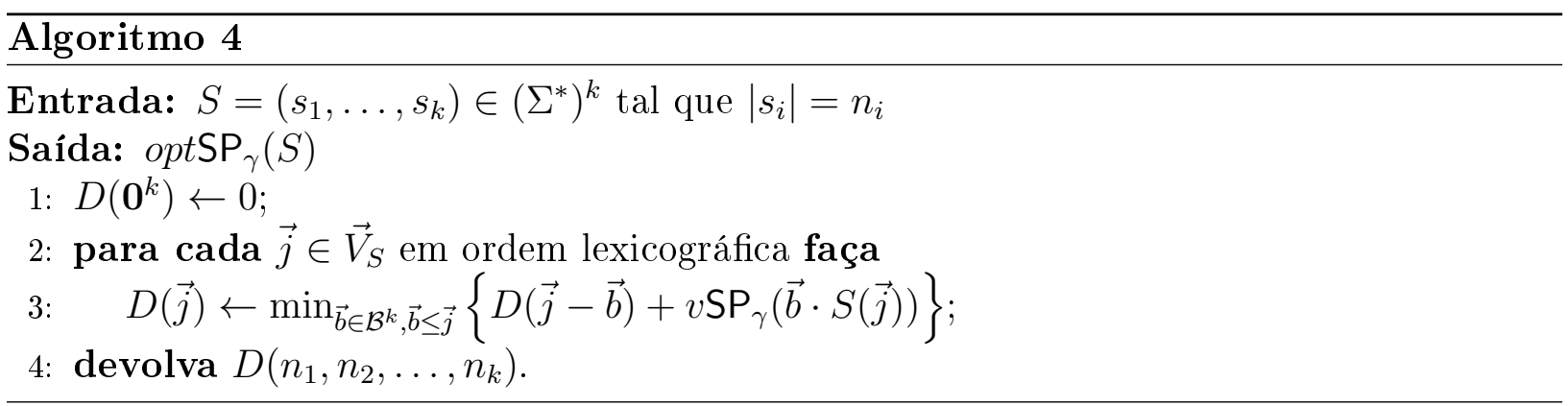

Se todas as sequências de $S$ são de comprimento $n$, o algoritmo gasta espaço $\Theta\left((n+1)^{k}\right)$, que é o espaço utilizado pela estrutura de dados para armazenar a tabela $D$. Para preencher cada entrada da tabela, o algoritmo examina $\Theta\left(2^{k}\right)$ outras entradas no pior caso sendo que o exame de cada uma dessas entradas gasta tempo $\Theta\left(k^{2}\right)$. Portanto, o tempo total consumido é $O\left(2^{k} k^{2}(n+1)^{k}\right)$.

Alguns métodos, como de Carrillo e Lipman [CL88], são mais eficientes quando as sequências são "parecidas" pois não calcula todas as entradas de $D$. No pior caso do caso geral, entretanto, o espaço e tempo gastos permanecem assintoticamente iguais.

\subsubsection{Complexidade do problema}

Para o estudo da complexidade consideramos a versão de decisão do problema.

Problema 4. AVSd: Alinhamento de Várias Sequências (Multiple sequence alignment)

Dados $S \in\left(\Sigma^{*}\right)^{k}$ e um inteiro positivo $C$, decidir se existe um alinhamento $A$ de $S$ tal que $v \operatorname{SP}_{\gamma}(A) \leq C$, onde $\gamma$ é uma matriz de pontuação fixa com entradas inteiras.

Nesta seção, dada uma matriz de pontuação $\gamma$ para um $\Sigma$, dizemos que uma matriz de pontuação $\delta$ para um $\Sigma^{\prime}$ é supermatriz de $\gamma$ se $\Sigma \subseteq \Sigma^{\prime}$ e $\gamma_{\mathrm{a} \rightarrow \mathrm{b}}=\delta_{\mathrm{a} \rightarrow \mathrm{b}}$ para cada a, b $\in \Sigma$.

Wang e Jiang [WJ94] mostraram que o Problema AVSd é NP-completo mas em sua redução os autores usaram matrizes que são supermatrizes de uma matriz de pontuação particular que não possui a propriedade reflexiva de uma métrica em $\Sigma_{\sqcup}$. Descrevemos a seguir essa redução.

\section{Redução de Wang e Jiang [WJ94]}

Uma supersequência de uma sequência $s(1) \ldots s(n) \in \Sigma^{*}$, onde cada $s(i) \in \Sigma$, é uma sequência na forma $t_{1} s(1) t_{2} s(2) t_{3} \ldots t_{n} s(n) t_{n+1}$, onde cada $t_{i} \in \Sigma^{*}$. 
Problema 5. SC-Mín: Supersequência Comum de Menor Comprimento (Shortest Common Supersequence Problem)

Dados um conjunto de sequências $\mathcal{S}=\left\{s_{1}, \ldots, s_{k}\right\}$ e um inteiro positivo $L$, decidir se existe uma sequência $s \in \Sigma^{*}$ tal que $s$ é supersequência de cada $s_{i} \in \mathcal{S}$ e $|s| \leq L$.

O Problema SC-Mín é NP-completo quando $|\Sigma|=2$ [RU81]. A seguir descrevemos uma redução do Problema SC-Mín ao Problema AVSd.

Sejam $\Sigma^{\text {SC-Min }}=\{0,1\}$ um alfabeto para uma instância $\left(\mathcal{S}=\left\{s_{1}, \ldots, s_{k}\right\}, L\right)$ de SCMín, $n_{i}=\left|s_{i}\right|$ e $N=\sum_{i} n_{i}$. Claramente a resposta do problema para a instância $(\mathcal{S}, L)$ é Não se $L<\max _{i}\left\{n_{i}\right\}$ e a resposta é Sim se $L \geq N$. Assumimos então que $\max _{i}\left\{n_{i}\right\} \leq L<N$.

Definimos $L+1$ instâncias $\left(S_{p}, C\right)$ de AVSd, para $p=0,1, \ldots, L$, onde $C$ é uma constante fixa igual a $k N+(k+1) L$. O alfabeto e a matriz de pontuação considerados são $\Sigma^{\mathbf{A V S}}=$ $\{0,1, a, b\}$ e

$\gamma=$\begin{tabular}{c|ccccc} 
& 0 & 1 & $a$ & $b$ & $\sqcup$ \\
\hline 0 & 2 & 2 & 1 & 2 & 1 \\
1 & 2 & 2 & 2 & 1 & 1 \\
$a$ & 1 & 2 & 0 & 2 & 1 \\
$b$ & 2 & 1 & 2 & 0 & 1 \\
& 1 & 1 & 1 & 1 &
\end{tabular}

respectivamente. Os símbolos em $\{a, b\}$ são chamados de letras e os símbolos em $\{0,1\}$ são chamados de símbolos numéricos. Note que as propriedades de não-negatividade, positividade, desigualdade triangular e simetria estão presentes em $\left(\Sigma^{\mathbf{A V S}}, \gamma\right)$, mas a propriedade reflexibilidade não está (pois $\gamma_{0 \rightarrow 0}=\gamma_{1 \rightarrow 1} \neq 0$ ), o que implica que $\gamma$ não é uma métrica em $\Sigma$. Para cada $p$, ponha $S_{p}=\left(s_{1}, \ldots, s_{k}, s_{k+1}=a^{p}, s_{k+2}=b^{L-p}\right)$.

Verificamos a seguir nesta seção que a resposta do Problema SC-Mín para instância $(\mathcal{S}, L)$ é Sim se e somente se a resposta do Problema AVSd para a instâncias $\left(S_{p}, C\right)$ é Sim para algum $p$. Para isto, mostramos um alinhamento com pontuação $C$ para algum $S_{p}$ quando existe uma supersequência comum das sequências de $\mathcal{S}$ de comprimento menor ou igual a $L$, e mostramos que qualquer alinhamento de qualquer $(k+2)$-upla $S_{p}$ tem pontuação superior a $C$ quando não existe uma supersequência comum das sequências de $\mathcal{S}$ de comprimento menor ou igual a $L$.

Verificamos pelo lema a seguir que qualquer alinhamento induzido $A_{\{1, \ldots, k\}}$ de qualquer $S_{p}$ possui pontuação constante.

Lema 30. Seja $A$ um alinhamento de $S_{p}$, para algum $p$. Então, $v \mathrm{SP}_{\gamma}\left(A_{\{1, \ldots, k\}}\right)=(k-1) N$.

Prova. Sejam $A$ um alinhamento de $S_{p}$, para algum $p$, e $h, i$ inteiros tais que $1 \leq h<i \leq k$. Seja $m$ a quantidade de símbolos de $s_{h}$ alinhados com símbolos de $s_{i}$. Portanto, $m-n_{h}$ símbolos de $s_{h}$ estão alinhados com $\sqcup$ e $m-n_{i}$ símbolos de $s_{i}$ estão alinhados com $\longleftarrow$ em $A_{\{h, i\}}$. Desde que $s_{h}$ e $s_{i}$ contêm somente símbolos numéricos e que $\gamma_{0 \rightarrow 0}=\gamma_{0 \rightarrow 1}=\gamma_{1 \rightarrow 0}=\gamma_{1 \rightarrow 1}=2$ e que $\gamma_{0 \rightarrow \sqcup}=\gamma_{\sqcup \rightarrow 0}=\gamma_{1 \rightarrow_{\llcorner}}=\gamma_{\sqcup \rightarrow 1}=1$, segue que

$$
v \mathrm{~A}_{\gamma}\left(A_{\{h, i\}}\right)=\left(n_{h}-m\right) \cdot 1+\left(n_{i}-m\right) \cdot 1+m \cdot 2=n_{h}+n_{i} .
$$

Logo,

$$
v \mathrm{SP}_{\gamma}\left(A_{\{1, \ldots, k\}}\right)=\sum_{h=1}^{k-1} \sum_{i<h}^{k} v \mathrm{~A}_{\gamma}\left(A_{\{h, i\}}\right)=\sum_{h=1}^{k-1} \sum_{i<h}^{k}\left(n_{h}+n_{i}\right)=(k-1) \sum_{h=1}^{k} n_{h}=(k-1) N .
$$

A duplicação da coluna $j$ em um alinhamento consiste na operação de inserção, em cada 
sequência $s^{\prime}$ do alinhamento, de um símbolo ${ }_{\sqcup}$ antes ou depois de $s^{\prime}(j)$ substituindo assim a coluna $j$ por outras duas, ou seja, substituindo cada sequência $s^{\prime}$ pela sequência

$$
s^{\prime}(1) \ldots s^{\prime}(j-1) s^{\prime}(j) s^{\prime}(j+1) \ldots s^{\prime}\left(\left|s^{\prime}\right|\right) \text { ou } s^{\prime}(1) \ldots s^{\prime}(j-1) s^{\prime}(j)_{\varsigma^{\prime}} s^{\prime}(j+1) \ldots s^{\prime}\left(\left|s^{\prime}\right|\right) .
$$

As novas colunas após a duplicação da coluna $j$ são as colunas $j$ e $j+1$ e cada uma delas não pode ter somente símbolos iguais a $\mathrm{L}^{\text {. }}$

Uma coluna $c$ de um alinhamento de $S_{p}$ é desejável se ela contém somente 0's, $a$ 's e ¿'s ou contém somente 1's, b's e ¿'s. Se a coluna $c$ não é desejável, então ela é indesejável. Note que se $A$ é um alinhamento sem colunas indesejáveis, então $|A| \geq L$ pois os símbolos de $a^{p}$ não podem estar alinhados com símbolos de $b^{L-p}$. Uma duplicação desejável de uma coluna indesejável $c=\left[c_{1}, \ldots, c_{k+2}\right]$ produz duas novas colunas $c^{\prime}=\left[c_{1}^{\prime}, \ldots, c_{k+2}^{\prime}\right]$ e $c^{\prime \prime}=$ $\left[c_{1}^{\prime \prime}, \ldots, c_{k+2}^{\prime \prime}\right]$ tais que: se $c_{i}=0$ ou $c_{i}=a$, então $c_{i}^{\prime}=c_{i}$ e $c_{i}^{\prime \prime}={ }_{\lrcorner}$; se $c_{i}=1$ ou $c_{i}=b$, então $c_{i}^{\prime}={ }_{\longleftarrow}$ e $c_{i}^{\prime \prime}=c_{i}$; e se $c_{i}={ }_{\iota}$, então $c_{i}^{\prime}=c_{i}^{\prime \prime}={ }$. Os seguintes resultados mostram que duplicação desejável não altera a pontuação de um alinhamento.

Lema 31. Seja $A$ um alinhamento de $S_{p}$ para algum $p$ com $q>0$ colunas indesejáveis. Se $c$ é uma coluna indesejável, então a duplicação desejável de $c$ produz um alinhamento $A^{\prime}$ com $q-1$ colunas indesejáveis e $v \mathrm{SP}_{\gamma}(A)=v \mathrm{SP}_{\gamma}\left(A^{\prime}\right)$.

Prova. Suponha que $c$ é uma coluna indesejável de $A$. Denotamos por $r_{\sigma}$ a quantidade de símbolos iguais a $\sigma$ em $c$. Sendo $\gamma$ simétrica, a pontuação-SP da coluna $c$ é

$$
\begin{aligned}
v \mathrm{SP}_{\gamma}(c)= & 2\left(\begin{array}{c}
r_{0} \\
2
\end{array}\right)+2\left(\begin{array}{c}
r_{1} \\
2
\end{array}\right)+2\left(r_{0} r_{1}+r_{0} r_{b}+r_{1} r_{a}+r_{a} r_{b}\right) \\
& +\left(r_{0} r_{a}+r_{0} r_{\sqcup}+r_{1} r_{b}+r_{1} r_{\sqcup}+r_{a} r_{\sqcup}+r_{b} r_{\sqcup}\right) .
\end{aligned}
$$

Sejam $c^{\prime}$ e $c^{\prime \prime}$ as novas colunas obtidas pela duplicação desejável de $c$. Assim, a coluna $c^{\prime}$ fica com $r_{0}$ símbolos iguais a $0, r_{a}$ símbolos iguais a $a$ e $r_{1}+r_{b}+r_{\sqcup}$ símbolos iguais a , e similarmente, a coluna $c_{i}^{\prime \prime}$ fica com $r_{1}$ símbolos iguais a $1, r_{b}$ símbolos iguais a $b$ e $r_{0}+r_{a}+r_{\sqcup}$ símbolos iguais a $\sqcup$. Segue que,

$$
\begin{aligned}
& v \mathrm{SP}_{\gamma}\left(c^{\prime}\right)=2\left(\begin{array}{c}
r_{0} \\
2
\end{array}\right)+r_{0}\left(r_{1}+r_{b}+r_{\sqcup}\right)+r_{0} r_{a}+r_{a}\left(r_{1}+r_{b}+r_{\sqcup}\right) \\
& v \mathrm{SP}_{\gamma}\left(c^{\prime \prime}\right)=2\left(\begin{array}{c}
r_{1} \\
2
\end{array}\right)+r_{1}\left(r_{0}+r_{a}+r_{\sqcup}\right)+r_{1} r_{b}+r_{b}\left(r_{0}+r_{a}+r_{\sqcup}\right) .
\end{aligned}
$$

Segue que $v \mathrm{SP}_{\gamma}(c)=v \mathrm{SP}_{\gamma}\left(c^{\prime}\right)+v \mathrm{SP}_{\gamma}\left(c^{\prime \prime}\right)$. Como as demais colunas de $A$ são colunas de $A^{\prime}$ e vice-versa, segue que $v \operatorname{SP}_{\gamma}(A)=v \mathrm{SP}_{\gamma}\left(A^{\prime}\right)$ e que $A^{\prime}$ possui exatamente uma coluna indesejável a menos que $A$.

Claramente, vale o seguinte corolário do Lema 31.

Corolário 32. Se $A$ é um alinhamento de $S_{p}$ para algum $p$, então existe um alinhamento $A^{\prime}$ de $S_{p}$ sem colunas indesejáveis tal que $v \mathrm{SP}_{\gamma}(A)=v \mathrm{SP}_{\gamma}\left(A^{\prime}\right)$.

O seguinte resultado determina a pontuação de um alinhamento sem colunas indesejáveis que possui uma letra em cada coluna.

Lema 33. Seja $A$ um alinhamento de $S_{p}$ sem colunas indesejáveis. Se em toda coluna de $A$ há exatamente uma letra ( $a$ ou $b)$, então $v \mathrm{SP}_{\gamma}(A)=k N+(k+1) L$; caso contrário $v \mathrm{SP}_{\gamma}(A)>k N+(k+1) L$. 
Prova. Seja $A=\left[s_{1}^{\prime}, \ldots, s_{k}^{\prime}, s_{k+1}^{\prime}, s_{k+2}^{\prime}\right]$. Como $A$ não possui colunas indesejáveis, temos que $|A|=L+q$, onde $q \geq 0$ é a quantidade de colunas que não têm letra. Segue que, se em toda coluna de $A$ há exatamente uma letra, então $q=0$. Caso contrário, $q>0$.

A pontuação-SP do alinhamento $A$ é

$$
v \mathrm{SP}_{\gamma}(A)=v \mathrm{SP}_{\gamma}\left(A_{\{1, \ldots, k\}}\right)+\sum_{j=1}^{L+q}\left(\gamma_{s_{k+1}^{\prime}(j) \rightarrow s_{k+2}^{\prime}(j)}+\sum_{i} \gamma_{s_{i}^{\prime}(j) \rightarrow s_{k+1}^{\prime}(j)}+\sum_{i} \gamma_{s_{i}^{\prime}(j) \rightarrow s_{k+2}^{\prime}(j)}\right) .
$$

Ponha

$$
X_{j}=\gamma_{s_{k+1}^{\prime}(j) \rightarrow s_{k+2}^{\prime}(j)}+\sum_{i} \gamma_{s_{i}^{\prime}(j) \rightarrow s_{k+1}^{\prime}(j)}+\sum_{i} \gamma_{s_{i}^{\prime}(j) \rightarrow s_{k+2}^{\prime}(j)}
$$

que é a contribuição de $a^{p}, b^{L-p}$ na coluna $j$ na pontuação-SP.

Sejam $c_{1}, c_{2}, \ldots, c_{L}$ as colunas com letra e $c_{L+1}, \ldots, c_{L+q}$ as colunas sem letra e seja $r_{j}$ a quantidade de símbolos numéricos na coluna $c_{j}$. Se $j \leq L$, ou seja, $c_{j}$ possui letra, então $X_{j}=(k+1)+r_{j}$, e se $j>L$, então $X_{j}=2 r_{j}$. Portanto,

$$
\sum_{j=1}^{L+q} X_{j}=\sum_{j=1}^{L} X_{j}+\sum_{j=L+1}^{L+q} X_{j}=\sum_{j=1}^{L}\left((k+1)+r_{j}\right)+\sum_{j=L+1}^{L+q} 2 r_{j}=L(k+1)+N+\sum_{j=L+1}^{L+q} r_{j} .
$$

Como pelo Lema $30 v \mathrm{SP}_{\gamma}\left(A_{\{1, \ldots, k\}}\right)=(k-1) N$, segue que

$$
v \mathrm{SP}_{\gamma}(A)=v \mathrm{SP}_{\gamma}\left(A_{\{1, \ldots, k\}}\right)+\sum_{j} X_{j}=k N+L(k+1)+\sum_{j=L+1}^{L+q} r_{j} .
$$

Suponha que em $A$ toda coluna tem exatamente uma letra. Logo, $q=0$ e, portanto, $\sum_{j=L+1}^{L+q} r_{j}=0$. Segue que

$$
v \mathrm{SP}_{\gamma}(A)=k N+(k+1) L+\sum_{j=L+1}^{L+q} r_{j}=k N+(k+1) L
$$

Portanto, se toda coluna de $A$ tem exatamente uma letra, então $v \mathrm{SP}_{\gamma}(A)=k N+(k+1) L$.

Caso contrário, $q>0$. Logo, desde que uma coluna $c_{j}$ não pode possuir unicamente símbolos iguais a ${ }$, temos que, se $j>L$, então $r_{j}>0$. Logo, se $q>0$, então $\sum_{j=L+1}^{L+q} r_{j}>0$. Segue que

$$
v \mathrm{SP}_{\gamma}(A)=k N+(k+1) L+\sum_{j=L+1}^{L+q} r_{j}>k N+(k+1) L .
$$

Portanto, se não é o caso de toda coluna de $A$ ter exatamente uma letra, $v \operatorname{SP}_{\gamma}(A)>k N+$ $(k+1) L$.

Lema 34. Sejam $t$ uma supersequência de comprimento mínimo de $\mathcal{S}=\left\{s_{1}, \ldots, s_{k}\right\}$ e $L$ um inteiro positivo. Existe um alinhamento de $S_{p}$ para algum $p$, sem colunas indesejáveis e com exatamente uma letra em cada coluna se e somente se $|t| \leq L$.

Prova. Suponha que existe um alinhamento $A$ de $S_{p}$ para algum $p$, sem colunas indesejáveis e com exatamente uma letra em cada coluna. Como há $p$ letras $a$ s e $L-p$ letras $b$ s, segue que $|A|=L$. Definimos uma sequência $t^{\prime}$ tal que o $j$-ésimo elemento de $t^{\prime}$ é 0 se a letra da $j$-ésima coluna de $A$ é $a$, e é 1 se a letra da $j$-ésima coluna de $A$ é $b$. Claramente $t^{\prime}$ corresponde a 
uma supersequência de $\mathcal{S}$ de comprimento $|A|=L$. Sendo $t$ uma supersequência de $\mathcal{S}$ de comprimento mínimo segue que $|t| \leq\left|t^{\prime}\right|=|A|=L$.

Reciprocamente, suponha $|t| \leq L$ para algum inteiro $L$ e considere $p$ a quantidade de símbolos iguais a 0 em $t$. Construímos um alinhamento $A$ para $S_{p}=\left(s_{1}, \ldots, s_{k}, a^{p}, b^{L-p}\right)$ de tal modo que, para cada $i$, os 0 's e os 1 s de $s_{i}$ fiquem alinhados com os 0 's e 1 's correspondentes aos símbolos da supersequência $t$; os $a$ 's e $|t|-p$ símbolos $b$ 's de $a^{p}$ e de $b^{L-p}$ fiquem alinhados com os 0 's e 1's correspondentes em $t$; os demais $L-|t|$ símbolos iguais a $b$ ficam alinhados com ـ em colunas isoladas. Por exemplo, se as sequências são 0000000,01010010, 01010,011010 e $t=0010100100$ e $L=11$, um possível alinhamento construído usando as regras acima é

$$
\left[\begin{array}{ccccccccccc}
0 & 0 & \sqcup & 0 & \sqcup & 0 & 0 & \sqcup & 0 & \sqcup & 0 \\
0 & \sqcup & 1 & 0 & 1 & 0 & 0 & 1 & \sqcup & \sqcup & 0 \\
& 0 & 1 & 0 & 1 & 0 & \sqcup & \sqcup & \sqcup & \sqcup & \sqcup \\
0 & \sqcup & 1 & \sqcup & 1 & \sqcup & 0 & 1 & 0 & \sqcup & \sqcup \\
\hline \hline a & a & \sqcup & a & \sqcup & a & a & \sqcup & a & \sqcup & a \\
\sqcup & \sqcup & b & \sqcup & b & \sqcup & \sqcup & b & \sqcup & b & \sqcup \\
0 & 0 & 1 & 0 & 1 & 0 & 0 & 1 & 0 & - & 0
\end{array}\right]
$$

Logo, se $|t| \leq L$, então existe um alinhamento de $S_{p}$ para algum $p$, sem colunas indesejáveis e com exatamente uma letra em cada coluna.

Teorema 35. O Problema AVSd é NP-completo para a matriz

$\gamma=$\begin{tabular}{c|ccccc} 
& 0 & 1 & $a$ & $b$ & $\sqcup$ \\
\hline 0 & 2 & 2 & 1 & 2 & 1 \\
1 & 2 & 2 & 2 & 1 & 1 \\
$a$ & 1 & 2 & 0 & 2 & 1 \\
$b$ & 2 & 1 & 2 & 0 & 1 \\
& 1 & 1 & 1 & 1 &
\end{tabular}.

Prova. O Problema AVSd está em NP pois, sendo $(S, C)$ uma instância de AVSd, podemos verificar em tempo polinomial se um alinhamento $A$ de $S$ é tal que $v \mathrm{SP}_{\gamma}(A) \leq C$.

A redução acima do Problema SC-Mín ao Problema AVSd claramente é polinomial. Basta verificar então que a resposta da instância $(\mathcal{S}, L)$ para o Problema SC-Mín é Sim se e somente se a resposta de alguma das $L+1$ instâncias $\left(S_{p}, C\right)$ para o Problema AVSd é Sim.

Suponha, então que a resposta da instância $(\mathcal{S}, L)$ para o Problema SC-Mín é Sim. Então, existe uma supersequência $t$ de $S$ de comprimento $|t| \leq L$. Segue do Lema 34 que, para algum $p$, existe um alinhamento $A$ de $S_{p}$, sem colunas indesejáveis e que possui letras em todas as colunas. Segue do Lema 33 que $v \mathrm{SP}_{\gamma}(A)=k N+(k+1) L$. Segue que a resposta da instância $\left(S_{p}, C\right)$ para o Problema AVSd é Sim.

Suponha, então que, para algum $j$, a resposta da instância $\left(S_{p}, C\right)$ para o Problema AVSd é Sim. Logo, existe um alinhamento $A$ de $S_{p}$ tal que $v \operatorname{SP}_{\gamma}(A) \leq C=k N+(k+1) L$. O Corolário 32 garante que existe um alinhamento $A^{\prime}$ de $S_{p}$ sem colunas indesejáveis e $v \mathrm{SP}_{\gamma}\left(A^{\prime}\right)=v \mathrm{SP}_{\gamma}(A)$. Segue do Lema 33 que em cada coluna de $A^{\prime}$ há exatamente uma letra. Segue do Lema 34 que existe uma supersequência de $\mathcal{S}$ de comprimento menor ou igual a $L$. Portanto, a resposta da $(S, L)$ para o Problema SC-Mín é Sim. 


\section{Outros resultados}

Bonizzoni e Vedova [BV01] mostraram que o Problema AVSd é NP-completo quando $|\Sigma| \geq 2$ e a matriz de pontuação é uma supermatriz de

$$
\gamma=\begin{array}{c|ccc} 
& \mathrm{a} & \mathrm{b} & \mathrm{c} \\
\hline \mathrm{a} & 0 & 1 & 2 \\
\mathrm{~b} & 1 & 0 & 2 \\
\sqcup & 2 & 2 &
\end{array},
$$

ou seja, para algumas matrizes que são métricas e que induzem métrica em optSP $\mathrm{P}_{\gamma}$.

Just [Jus01] mostrou que o problema é NP-completo para uma classe de matrizes de pontuação mais ampla que incluia inclusive as matrizes de Bonizzoni e Vedova (mas não as de Wang e Jiang) e que são supermatrizes de

$$
\gamma=\begin{array}{c|ccc} 
& \mathrm{a} & \mathrm{b} & \mathrm{c} \\
\hline \mathrm{a} & v_{\mathrm{a}} & u & y \\
\mathrm{~b} & u & v_{\mathrm{b}} & z \\
& y & z &
\end{array},
$$

onde $u>\max \left\{0, v_{\mathrm{a}}, v_{\mathrm{b}}\right\}, y>u$ e $z>0$.

Elias [Eli06] mostrou que o problema é NP-completo quando a matriz de pontuação é uma supermatriz de uma matriz que é métrica em $\Sigma_{\sqcup}$, e $\Sigma$ possui ao menos dois símbolos.

A Tabela 2.2 resume os resultados de NP-completude para o Problema AVSd estudados neste capítulo.

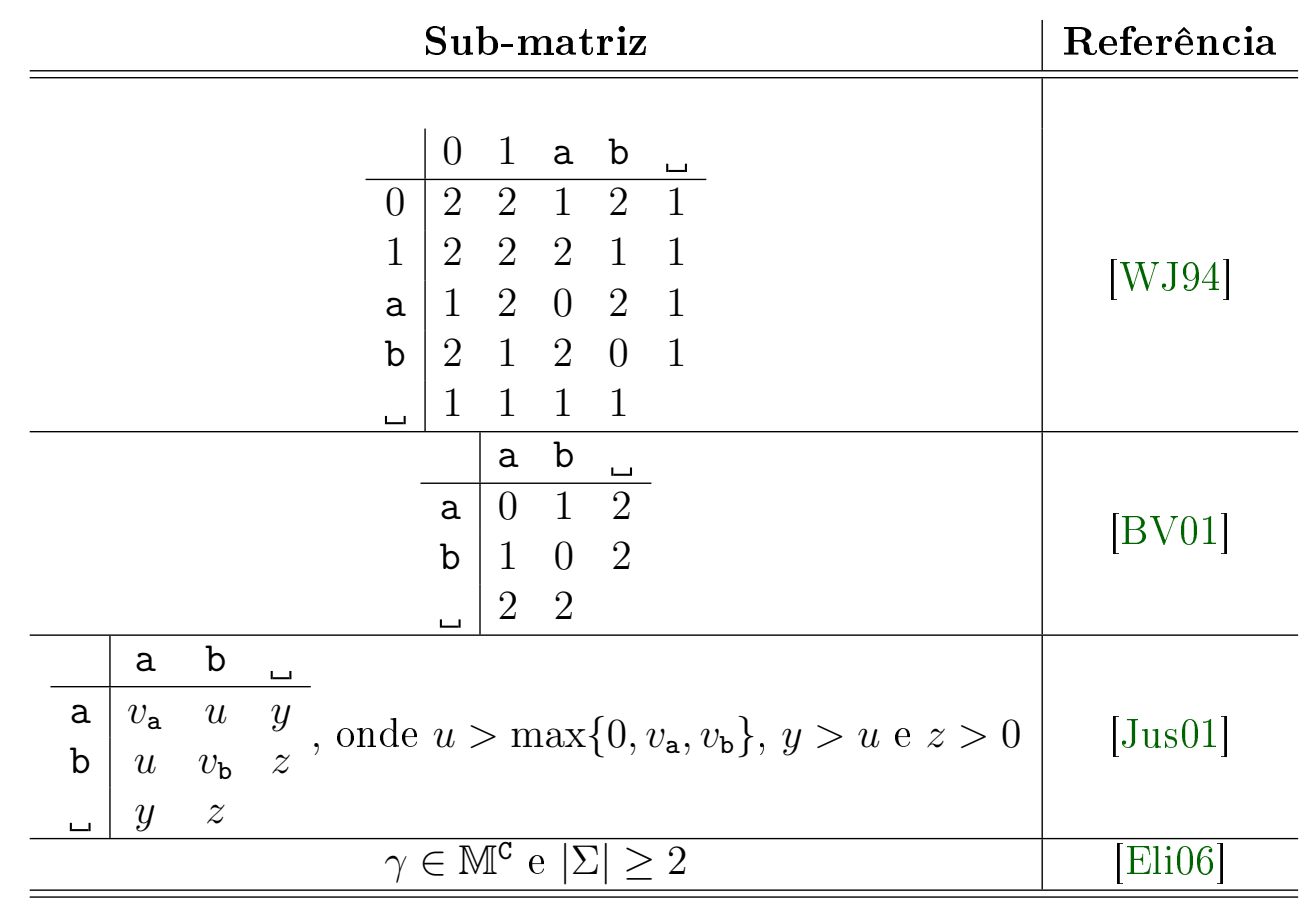

Tabela 2.2: Assumimos aqui que $\mathrm{a}, \mathrm{b} \in \Sigma$ e que a matriz de pontuação é uma supermatriz da sub-matriz dada. 


\section{Capítulo 3}

\section{Alinhamento Estendido}

Um alinhamento convencional de duas sequências representa um conjunto de operações de edição mas nem todo conjunto de operações de edição pode ser representado por um alinhamento convencional. Por exemplo, suponha que queremos representar as operações de edição cuja soma é mínima e que transforma a sequência a na sequência $b$ para a matriz de pontuação

$$
\gamma=\begin{array}{c|cccc} 
& \mathrm{a} & \mathrm{b} & \mathrm{c} & \mathrm{c} \\
\hline \mathrm{a} & 0 & 5 & 1 & 2 \\
\mathrm{~b} & 5 & 0 & 1 & 2 \\
\mathrm{c} & 1 & 1 & 0 & 2 \\
\mathrm{c} & 2 & 2 & 2 &
\end{array} .
$$

Há somente três maneiras de alinhar $(\mathrm{a}, \mathrm{b})$ de maneira convencional. Na primeira temos

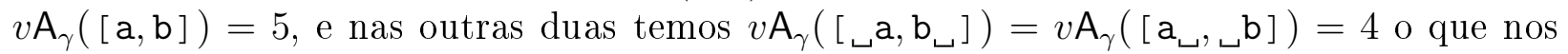
leva a concluir que opt $\mathrm{A}_{\gamma}(\mathrm{a}, \mathrm{b})=4$ e que $\left[\mathrm{a}_{\sqcup}, \mathrm{s}\right]$ é um alinhamento ótimo de $(\mathrm{a}, \mathrm{b})$ para o critério $v \mathrm{~A}_{\gamma}$. Entretanto, $\left[\mathrm{a}_{\sqcup}, \mathrm{b}\right.$ ] não representa qualquer conjunto de operações de edição de soma mínima, pois existe um de soma menor: editar a para c e, depois, c para b. Mas essa sequência não pode ser representada por um alinhamento convencional. Estendemos então o conceito de alinhamento para representar o conjunto de operações de edição. O alinhamento estendido "editar a para c e, depois, c para b" pode ser representado por

$$
\left[\begin{array}{l}
a \\
c \\
b
\end{array}\right] \text {. }
$$

Sejam $s, t \in \Sigma^{*}$. Um alinhamento estendido de $(s, t)$ é uma generalização do conceito de alinhamento. Qualquer conjunto de operações de edição que transforma $s$ em $t$ pode ser representado por essa estrutura. Formalmente, um alinhamento estendido de $(s, t)$ é uma $n$-upla $\left[c_{1}, c_{2}, \ldots, c_{n}\right]$, onde cada $c_{j}$ é uma sequência finita e não vazia com $m_{j}=\left|c_{j}\right|$ elementos em $\Sigma$ e com pelo menos um elemento em $\Sigma$. A sequência $s$ é a sequência obtida removendo os símbolos $\sqcup$ de $c_{1}(1) c_{2}(1) \ldots c_{n}(1)$, e $t$ é obtida removendo os símbolos $\sqcup$ de $c_{1}\left(m_{1}\right) c_{2}\left(m_{2}\right) \ldots c_{n}\left(m_{n}\right)$. Assim, por exemplo, [a, abcd, $\left.\mathrm{b}_{\lrcorner}, \sqcup \mathrm{d}, \mathrm{ca}, \_\mathrm{dabc}, \mathrm{abc}, \mathrm{dab}\right]$ é um alinhamento estendido de (aabcd, addaccb). Dizemos simplesmente alinhamento em vez de alinhamento estendido se o tipo de alinhamento está claro no contexto.

Se $A=\left[c_{1}, c_{2}, \ldots, c_{n}\right]$ é um alinhamento estendido de duas sequências, então dizemos que cada $c_{j}$ é a coluna do alinhamento e que $n=|A|$ é o comprimento do alinhamento $A$. 
A representação gráfica

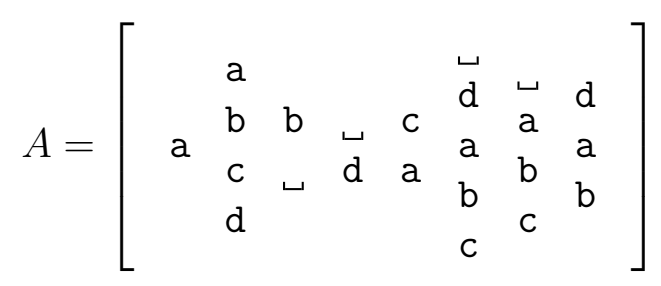

do alinhamento $A$ de (aabcd, addaccb) facilita a visualização das operações de edição que $A$ representa.

Em um alinhamento estendido cada coluna representa uma sequência de operações de edição que transforma um símbolo de $\Sigma_{\sqcup}$ em outro de $\Sigma_{\sqcup}$. A pontuação- $v \mathrm{E}_{\gamma}$ para editar a coluna $c_{j}$ ou simplesmente a pontuação- $v \mathrm{E}_{\gamma}$ de $c_{j}$ é

$$
v \mathrm{E}_{\gamma}\left(c_{j}\right)=\sum_{i=1}^{m_{j}-1} \gamma_{c_{j}(i) \rightarrow c_{j}(i+1)}
$$

e o critério $v \mathrm{E}_{\gamma}$ para pontuar o alinhamento $\left[c_{1}, \ldots, c_{n}\right]$ é

$$
v \mathrm{E}_{\gamma}\left(\left[c_{1}, \ldots, c_{n}\right]\right)=\sum_{j=1}^{n} v \mathrm{E}_{\gamma}\left(c_{j}\right) .
$$

Dizemos que o valor de $v \mathrm{E}_{\gamma}\left(\left[c_{1}, \ldots, c_{n}\right]\right)$ é a pontuação- $v \mathrm{E}_{\gamma}$ do alinhamento $\left[c_{1}, \ldots, c_{n}\right]$.

O conjunto de todos os alinhamentos estendidos de $(s, t)$ é denotado por $\mathcal{E}_{\{s, t\}}$ e definimos

$$
o p t \mathrm{E}_{\gamma}(s, t)=\min _{A \in \mathcal{E}_{\{s, t\}}}\left\{v \mathrm{E}_{\gamma}(A)\right\}
$$

Dizemos que um alinhamento estendido de $(s, t)$ de pontuação- $v \mathrm{E}_{\gamma}$ opt $\mathrm{E}_{\gamma}(s, t)$ é um alinhamento E-ótimo para $\gamma$. Enunciamos então o seguinte problema.

Problema 6. CME: Custo mínimo de edição

Dados $s, t \in \Sigma^{*}$, determinar opt $\mathrm{E}_{\gamma}(s, t)$, onde $\gamma$ é uma matriz fixa.

A matriz de pontuação $\gamma$ pode ser vista como um digrafo $D(\gamma)$ com peso nos arcos, onde o conjunto de vértices é $V(D)=\Sigma_{\sqcup}$ e o conjunto de arcos é $E(D)=\Sigma_{\sqcup} \times \Sigma_{\sqcup} \backslash\{(\sqcup, \sqcup)\}$ e custo $(a \rightarrow b)=\gamma_{a \rightarrow b}$ para cada $a \rightarrow b \in E(D)$. A Figura 3.1 mostra $D(\breve{\gamma})$ onde

$$
\gamma=\begin{array}{c|cccc} 
& \mathrm{a} & \mathrm{b} & \mathrm{c} & \mathrm{u} \\
\hline \mathrm{a} & 0 & 5 & 1 & 2 \\
\mathrm{~b} & 5 & 0 & 1 & 2 \\
\mathrm{c} & 1 & 1 & 0 & 2 \\
\mathrm{c} & 2 & 2 & 2 &
\end{array} .
$$

Relembrando os conceitos definidos no Capítulo 2, temos que uma sequência de arcos $P=\left(s(1) \rightarrow s(2)=e_{1}, \ldots, e_{n-1}=s(n-1) \rightarrow s(n)\right)$ em $D(\gamma)$ é um caminho de $s(1)$ a $s(n)$ de custo

$$
\operatorname{custo}(P)=\sum_{i=1}^{n-1} \text { custo }\left(e_{i}\right)
$$

se $s(1)=s(n)$, dizemos que $P$ é um ciclo; e se $P$ é um ciclo e custo $(P)<0$, dizemos que $P$ é um ciclo negativo. 


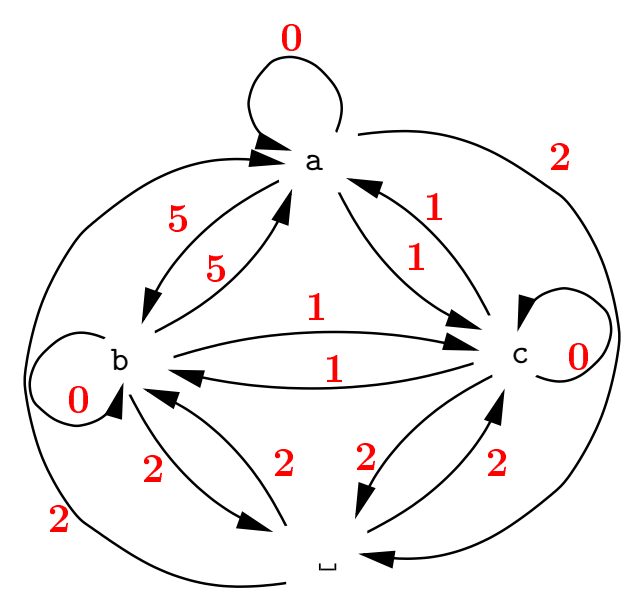

Figura 3.1: Digrafo $D(\gamma)$.

Pelo Lema 64 demonstrado no Capítulo 4, a pontuação- $v \mathrm{E}_{\gamma}$ de um alinhamento E-ótimo para $\gamma$ não é definida se existem ciclos negativos em $D(\gamma)$. Assumimos portanto que $D(\gamma)$ não possui ciclos negativos.

Um caminho ótimo de $s(1)$ a $s(n)$ em $D(\gamma)$ é um caminho de menor custo de $s(1)$ a $s(n)$ em $D(\gamma)$ e denotamos o seu custo por $\mathcal{P}_{\gamma}(s(1), s(n))$.

Dizemos que um alinhamento convencional $\left[s^{\prime}(1) \ldots s^{\prime}(n), t^{\prime}(1) \ldots t^{\prime}(n)\right]$ induz o alinhamento estendido $\left[s^{\prime}(1) t^{\prime}(1), s^{\prime}(2) t^{\prime}(2), \ldots, s^{\prime}(n) t^{\prime}(n)\right]$. Como vimos, nem sempre os valores de opt $\mathrm{E}_{\gamma}(s, t)$ e opt $\mathrm{A}_{\gamma}(s, t)$ são iguais. Entretanto, todo alinhamento convencional induz um alinhamento estendido de igual pontuação. Logo,

$$
o p t \mathrm{E}_{\gamma}(s, t) \leq \operatorname{opt} \mathrm{A}_{\gamma}(s, t)
$$

Mostramos a seguir como determinar uma matriz de pontuação $\delta$ de tal modo que $o p t \mathrm{E}_{\gamma}(s, t)=o p t \mathrm{~A}_{\delta}(s, t)$. A finalidade desta computação é determinar opt $\mathrm{E}_{\gamma}(s, t)$ usando algoritmos para computar opt $\mathrm{A}_{\delta}(s, t)$ gastando tempo adicional apenas para o pré-processamento da matriz de pontuação. A matriz de pontuação $\delta$ é definida a partir de $\gamma$. Para cada $\mathrm{a}, \mathrm{b} \in \Sigma_{\omega}$,

$$
\delta_{\mathrm{a} \rightarrow \mathrm{b}}=\mathcal{P}_{\gamma}(\mathrm{a}, \mathrm{b})
$$

A matriz de pontuação $\delta$ possui as seguintes propriedades.

Lema 36. Sejam $\gamma$ uma matriz de pontuação tal que $D(\gamma)$ não possui ciclo negativo, e $\delta$ uma matriz de pontuação definida a partir de $\gamma$ e conforme (3.2). Se a, b, c $\in \Sigma_{\sqcup}$, então

1. $\delta_{\mathrm{a} \rightarrow \mathrm{a}}=0 \mathrm{e}$

2. $\delta_{\mathrm{a} \rightarrow \mathrm{b}} \leq \delta_{\mathrm{a} \rightarrow \mathrm{c}}+\delta_{\mathrm{c} \rightarrow \mathrm{b}}$.

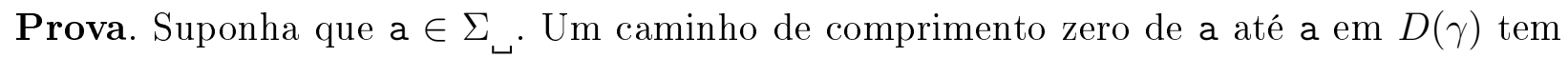
custo zero e, portanto, $\mathcal{P}_{\gamma}(\mathrm{a}, \mathrm{a}) \leq 0$. Como $D(\gamma)$ por definição não possui ciclo negativo, temos que qualquer caminho de a até a possui custo maior ou igual a zero e portanto $\mathcal{P}_{\gamma}(\mathrm{a}, \mathrm{a}) \geq 0$. Das observações feitas neste parágrafo, concluímos que $\mathcal{P}_{\gamma}(\mathrm{a}, \mathrm{a})=0$, o que implica que $\delta_{\mathrm{a} \rightarrow \mathrm{a}}=0$.

Suponha que a, b, c $\in \Sigma_{\sqcup}$. Por definição, os caminhos ótimos $P_{1}$ de a a c e $P_{2}$ de c a b em $D(\gamma)$ têm respectivamente custos $\mathcal{P}_{\gamma}(\mathrm{a}, \mathrm{c})=\delta_{\mathrm{a} \rightarrow \mathrm{c}}$ e $\mathcal{P}_{\gamma}(\mathrm{c}, \mathrm{b})=\delta_{\mathrm{c} \rightarrow \mathrm{b}}$. O caminho $P_{1} P_{2}$ 
definido pela concatenação de $P_{1}$ e $P_{2}$ é um caminho de a a b de custo $\mathcal{P}_{\gamma}(\mathrm{a}, \mathrm{c})+\mathcal{P}_{\gamma}(\mathrm{c}, \mathrm{b})$. Logo, desde que $\mathcal{P}_{\gamma}(\mathrm{a}, \mathrm{b})=\delta_{\mathrm{a} \rightarrow \mathrm{b}}$ é o custo do caminho ótimo de a para $\mathrm{b}$, segue que

$$
\delta_{\mathrm{a} \rightarrow \mathrm{b}}=\mathcal{P}_{\gamma}(\mathrm{a}, \mathrm{b}) \leq \operatorname{custo}\left(P_{1} P_{2}\right)=\mathcal{P}_{\gamma}(\mathrm{a}, \mathrm{c})+\mathcal{P}_{\gamma}(\mathrm{c}, \mathrm{b})=\delta_{\mathrm{a} \rightarrow \mathrm{c}}+\delta_{\mathrm{c} \rightarrow \mathrm{b}} .
$$

$\operatorname{Logo}, \delta_{\mathrm{a} \rightarrow \mathrm{b}} \leq \delta_{\mathrm{a} \rightarrow \mathrm{c}}+\delta_{\mathrm{c} \rightarrow \mathrm{b}}$

Lema 37. Sejam $\gamma$ uma matriz de pontuação tal que $D(\gamma)$ não possui ciclo negativo, $\delta$ uma matriz de pontuação definida a partir de $\gamma$ e conforme (3.2), e $s, t \in \Sigma^{*}$. Então, existe um alinhamento estendido $A^{\prime}$ de $(s, t)$ que é E-ótimo para $\delta$ e que também é induzido por um alinhamento convencional de $(s, t)$, ou seja, cada coluna de $A^{\prime}$ possui exatamente dois símbolos.

Prova. Seja $A=\left[c_{1}, \ldots, c_{n}\right]$ um alinhamento estendido de $(s, t)$ que é E-ótimo para $\delta$. Para cada coluna $c_{j}$ de $A$ definimos $c_{j}^{\prime}=\left[c_{j}(1), c_{j}\left(m_{j}\right)\right]$. Por construção, $\left[c_{1}^{\prime}, \ldots, c_{n}^{\prime}\right]$ é um alinhamento estendido que é induzido por um alinhamento convencional. Portanto, para provar o lema, basta mostrar que $v \mathrm{E}_{\delta}\left(A^{\prime}=\left[c_{1}^{\prime}, \ldots, c_{n}^{\prime}\right]\right)=o p t \mathrm{E}_{\delta}(s, t)$.

Para calcular $v \mathrm{E}_{\delta}\left(A^{\prime}\right)$, vamos primeiramente calcular a $v \mathrm{E}_{\delta}$-pontuação de $c_{j}^{\prime}$ para cada $j=1, \ldots, n$. Se $m_{j}=1$, então $c_{j}(1)=c_{j}\left(m_{j}\right)$ o que, desde que $\delta_{c_{j}(1) \rightarrow c_{j}(1)}=0$ pelo Item 1 do Lema 36, implica que $v \mathrm{E}_{\delta}\left(c_{j}^{\prime}\right)=\delta_{c_{j}(1) \rightarrow c_{j}\left(m_{j}\right)}=\delta_{c_{j}(1) \rightarrow c_{j}(1)}=0=v \mathrm{E}_{\delta}\left(c_{j}\right)$; se $m_{j}=2$, então $c_{j}^{\prime}=c_{j}$ e, portanto, $v \mathrm{E}_{\delta}\left(c_{j}^{\prime}\right)=v \mathrm{E}_{\delta}\left(c_{j}\right)$; e se $m>2$, então segue do Item 2 do Lema 36 que

$$
\begin{aligned}
v \mathrm{E}_{\delta}\left(c_{j}^{\prime}\right) & =\delta_{c_{j}(1) \rightarrow c_{j}\left(m_{j}\right)} \leq \delta_{c_{j}(1) \rightarrow c_{j}(2)}+\delta_{c_{j}(2) \rightarrow c_{j}\left(m_{j}\right)} \\
& \leq \delta_{c_{j}(1) \rightarrow c_{j}(2)}+\delta_{c_{j}(2) \rightarrow c_{j}(3)}+\delta_{c_{j}(3) \rightarrow c_{j}\left(m_{j}\right)} \leq \ldots \leq \sum_{i=1}^{n-1} \delta_{c_{j}(i) \rightarrow c_{j}(i+1)}=v \mathrm{E}_{\delta}\left(c_{j}\right) .
\end{aligned}
$$

Portanto,

$$
\begin{aligned}
v \mathrm{E}_{\delta}\left(A^{\prime}\right) & =\sum_{j} v \mathrm{E}_{\delta}\left(c_{j}^{\prime}\right)=\sum_{j: m_{j}=1} v \mathrm{E}_{\delta}\left(c_{j}^{\prime}\right)+\sum_{j: m_{j}=2} v \mathrm{E}_{\delta}\left(c_{j}^{\prime}\right)+\sum_{j: m_{j}>2} v \mathrm{E}_{\delta}\left(c_{j}^{\prime}\right) \\
& \leq \sum_{j: m_{j}=1} v \mathrm{E}_{\delta}\left(c_{j}\right)+\sum_{j: m_{j}=2} v \mathrm{E}_{\delta}\left(c_{j}\right)+\sum_{j: m_{j}>2} v \mathrm{E}_{\delta}\left(c_{j}\right)=\sum_{j} v \mathrm{E}_{\delta}\left(c_{j}\right)=v \mathrm{E}_{\delta}(A),
\end{aligned}
$$

o que implica, sendo $v \mathrm{E}_{\delta}(A)=\operatorname{opt} \mathrm{E}_{\delta}(s, t)$, que $v \mathrm{E}_{\delta}\left(A^{\prime}\right) \leq v \mathrm{E}_{\delta}(A)=o p t \mathrm{E}_{\delta}(s, t)$. Logo, $v \mathrm{E}_{\delta}\left(A^{\prime}\right)=o p t \mathrm{E}_{\delta}(s, t)$.

O seguinte resultado mostra que opt $\mathrm{E}_{\gamma}(s, t)=o p t \mathrm{~A}_{\delta}(s, t)$.

Teorema 38. Sejam $\gamma$ uma matriz de pontuação tal que $D(\gamma)$ não possui ciclo negativo, $\delta$ uma matriz de pontuação definida a partir de $\gamma$ e conforme (3.2), e $s, t \in \Sigma^{*}$. Então,

$$
o p t \mathrm{E}_{\gamma}(s, t)=o p t \mathrm{E}_{\delta}(s, t)=o p t \mathrm{~A}_{\delta}(s, t) .
$$

Prova. Seja $\left[c_{1}, \ldots, c_{n}\right]$ um alinhamento estendido de $(s, t)$.

Suponha que $\left[c_{1}, \ldots, c_{n}\right]$ é E-ótimo para $\gamma$. Logo, opt $\mathrm{E}_{\gamma}(s, t)=\sum_{j=1}^{n} v \mathrm{E}_{\gamma}\left(c_{j}\right)$. Como $v \mathrm{E}_{\gamma}\left(c_{j}\right)$ para um inteiro $j$ arbitrário é o custo de um caminho de $c_{j}(1)$ a $c_{j}\left(m_{j}\right)$ em $D(\gamma)$ e $\mathcal{P}_{\gamma}\left(c_{j}(1), c_{j}\left(m_{j}\right)\right)$ é o custo de um caminho ótimo de $c_{j}(1)$ a $c_{j}\left(m_{j}\right)$ em $D(\gamma)$, temos que 
$v \mathrm{E}_{\gamma}\left(c_{j}\right) \geq \mathcal{P}_{\gamma}\left(c_{j}(1), c_{j}\left(m_{j}\right)\right)=\delta_{c_{j}(1) \rightarrow c_{i}\left(m_{j}\right)}$. Segue daí, desde que $j$ é arbitrário, que

$$
\begin{aligned}
o p t \mathrm{E}_{\gamma}(s, t) & =\sum_{j=1}^{n} v \mathrm{E}_{\gamma}\left(c_{j}\right) \geq \sum_{j=1}^{n} \mathcal{P}_{\gamma}\left(c_{j}(1), c_{j}\left(m_{j}\right)\right)=\sum_{j=1}^{n} \delta_{c_{j}(1) \rightarrow c_{j}\left(m_{j}\right)} \\
& =v \mathrm{E}_{\delta}\left(\left[\begin{array}{ccc}
c_{1}(1) & \ldots & c_{n}(1) \\
c_{1}\left(m_{1}\right) & \ldots & c_{n}\left(m_{n}\right)
\end{array}\right]\right) \geq \operatorname{opt} \mathrm{E}_{\delta}(s, t) .
\end{aligned}
$$

Portanto, opt $\mathrm{E}_{\gamma}(s, t) \geq o p t \mathrm{E}_{\delta}(s, t)$.

Suponha agora que $\left[c_{1}, \ldots, c_{n}\right]$ é E-ótimo para $\delta$ e que é induzido por um alinhamento convencional que o Lema 37 garante existir. Desde que cada $c_{j}$ possui 2 símbolos, segue que $v \mathrm{E}_{\delta}\left(c_{j}\right)=\delta_{c_{j}(1) \rightarrow c_{j}\left(m_{j}\right)}=\mathcal{P}_{\gamma}\left(c_{j}(1), c_{j}\left(m_{j}\right)\right)$ para cada $j$ o que implica que $v \mathrm{E}_{\delta}\left(\left[c_{1}, \ldots, c_{n}\right]\right)=\sum_{j=1}^{n} \mathcal{P}_{\gamma}\left(c_{j}(1), c_{j}\left(m_{j}\right)\right)$. Um alinhamento estendido obtido a partir do alinhamento $\left[c_{1}, \ldots, c_{n}\right]$ substituindo cada coluna $c_{j}$ por uma sequência $c_{j}^{\prime}$ de símbolos que induz um caminho ótimo de $c_{j}(1)$ a $c_{j}\left(m_{j}\right)$ em $D(\gamma)$ é um alinhamento estendido de $(s, t)$ e $v \mathrm{E}_{\gamma}\left(\left[c_{1}^{\prime}, \ldots, c_{n}^{\prime}\right]\right)=\sum_{j=1}^{n} \mathcal{P}_{\gamma}\left(c_{j}(1), c_{j}\left(m_{j}\right)\right)$. Segue dos argumentos apresentados neste parágrafo que

$o p t \mathrm{E}_{\gamma}(s, t) \leq v \mathrm{E}_{\gamma}\left(\left[c_{1}^{\prime}, \ldots, c_{n}^{\prime}\right]\right)=\sum_{j=1}^{n} \mathcal{P}_{\gamma}\left(c_{j}(1), c_{j}\left(m_{j}\right)\right)=v \mathrm{E}_{\delta}\left(\left[c_{1}, \ldots, c_{n}\right]\right)=o p t \mathrm{E}_{\delta}(s, t)$.

Portanto, opt $\mathrm{E}_{\gamma}(s, t) \leq o p t \mathrm{E}_{\delta}(s, t)$.

As conclusões combinadas obtidas nos dois parágrafos anteriores nos permitem concluir que $o p t \mathrm{E}_{\gamma}(s, t)=o p t \mathrm{E}_{\delta}(s, t)$.

Pelo Lema 37, existe um alinhamento estendido $A$ de $(s, t)$ que é E-ótimo para $\delta$ e que também é induzido por um alinhamento convencional $A^{\prime}$ de $(s, t)$. Como todo alinhamento convencional induz um alinhamento estendido de igual pontuação, temos que $v \mathrm{E}_{\delta}(A)=$ $v \mathrm{~A}_{\delta}\left(A^{\prime}\right)$. Sendo $A$ é E-ótimo para $\delta$, segue que opt $\mathrm{E}_{\delta}(s, t)=v \mathrm{E}_{\delta}(A)=v \mathrm{~A}_{\delta}\left(A^{\prime}\right) \geq$ opt $\mathrm{A}_{\delta}(s, t)$. $\operatorname{Logo}, \operatorname{opt}_{\delta}(s, t) \geq o p t \mathrm{~A}_{\delta}(s, t)$. Desde que, por (3.1), opt $\mathrm{E}_{\delta}(s, t) \leq o p t \mathrm{~A}_{\delta}(s, t)$, segue que $o p t \mathrm{E}_{\delta}(s, t)=\operatorname{opt} \mathrm{A}_{\delta}(s, t)$.

Segue que

$$
o p t \mathrm{E}_{\gamma}(s, t)=o p t \mathrm{E}_{\delta}(s, t)=o p t \mathrm{~A}_{\delta}(s, t)
$$

Como consequência do Teorema 38, podemos determinar opt $\mathrm{E}_{\gamma}(s, t)$ usando um algoritmo que computa o valor de opt $\mathrm{A}_{\delta}(s, t)$. O tempo gasto por esse algoritmo é o tempo gasto para computar a matriz $\delta$ mais o tempo gasto para computar o valor de opt $\mathrm{A}_{\delta}(s, t)$.

Para computar a matriz de pontuação $\delta$ basta resolver o conhecido problema de determinar o custo dos caminhos mais curtos de todos os pares de vértices do digrafo $D(\gamma)$. O algoritmo de Floyd-Warshall faz esse trabalho gastando tempo $\Theta\left(\left|\Sigma_{\nu}\right|^{3}\right)$ [Flo62] [War62]. Portanto, desde que $\Sigma$ é constante e não faz parte da entrada do problema CME, o tempo gasto no pré-processamento é $\Theta(1)$.

Conforme visto no Capítulo 2, se $|s|=|t|=n$, o valor de opt $\mathrm{A}_{\delta}(s, t)$ pode ser computado em tempo $T_{\mathrm{APS}}(n)$. Portanto, um algoritmo que utiliza essas estratégias combinadas gasta tempo $O\left(T_{\mathrm{APS}}(n)\right)$ para computar opt $\mathrm{E}_{\gamma}(s, t)$. Além disso, o algoritmo de Floyd-Warshall pode também computar uma estrutura que é utilizada para obter os caminhos ótimos em tempo proporcional ao comprimento do caminho. Então, se, além de $\operatorname{opt} \mathrm{A}_{\delta}(s, t)$, o alinhamento convencional é computado, a estrutura de Floyd-Warshall pode também computar, a partir do alinhamento convencional, o alinhamento estendido em tempo $O\left(T_{\mathrm{APS}}(n)\right)$. 


\section{Capítulo 4}

\section{Matrizes que induzem métricas}

Para um dado conjunto $S$, dizemos que a função $f: S \times S \rightarrow \mathbb{R}$ é uma métrica se $f$ satisfaz simultaneamente

1. $f(s, s)=0$ (reflexibilidade),

2. $f(s, t) \geq 0$ (não-negatividade),

3. $f(s, t)>0$ se $s \neq t$ (positividade),

4. $f(s, t)=f(t, s)($ simetria $)$ e

5. $f(s, u) \leq f(s, t)+f(t, u)$ (desigualdade triangular $)$

para cada $s, t, u \in S$.

Outras classes de funções mais gerais do que métricas requerem que algumas, mas nem todas, propriedades acima sejam satisfeitas. Por exemplo, para um dado conjunto $S$, dizemos que $f$ é uma pramétrica [AP90] se $f(s, s)=0$ e $f(s, t) \geq 0$ para cada $s, t \in S$. A Tabela 4.1 mostra, além de métrica $(\mathcal{M})$, as propriedades de algumas dessas classes: pramétrica $(\mathcal{P r})$, semimétrica $(\mathcal{S})$ [Wil31b], hemimétrica também dita pseudoquasimétrica $(\mathcal{H})$ [Kim68], pseudométrica $(\mathcal{P})$ [SS70] e quasimétrica $(Q)$ [Wil31a]. A Figura 4.1 mostra graficamente a relação entre essas classes de funções.

\begin{tabular}{|c||c|c|c|c|c|c|}
\hline Propriedade $\backslash$ Função & $\mathcal{P r}$ & $\mathcal{S}$ & $\mathcal{H}$ & $\mathcal{P}$ & $\mathcal{Q}$ & $\mathcal{M}$ \\
\hline \hline$f(s, s)=0$ & $\mathrm{x}$ & $\mathrm{x}$ & $\mathrm{x}$ & $\mathrm{x}$ & $\mathrm{x}$ & $\mathrm{x}$ \\
\hline$f(s, t) \geq 0$ & $\mathrm{x}$ & $\mathrm{x}$ & $\mathrm{x}$ & $\mathrm{x}$ & $\mathrm{x}$ & $\mathrm{x}$ \\
\hline$f(s, t)>0$ para $s \neq t$ & & $\mathrm{x}$ & & & $\mathrm{x}$ & $\mathrm{x}$ \\
\hline$f(s, t)=f(t, s)$ & & $\mathrm{x}$ & & $\mathrm{x}$ & & $\mathrm{x}$ \\
\hline$f(s, u) \leq f(s, t)+f(t, u)$ & & & $\mathrm{x}$ & $\mathrm{x}$ & $\mathrm{x}$ & $\mathrm{x}$ \\
\hline
\end{tabular}

Tabela 4.1: Cada célula marcada com $\mathrm{x}$ na linha $i$, coluna $j$ representa que uma função da classe de funçôes $j$ requer, por definição, que a propriedade i seja satisfeita.

Se opt $\mathrm{A}_{\gamma}\left(\right.$ opt $\mathrm{N}_{\gamma}$, opt $\left.\mathrm{E}_{\gamma}\right)$ possui uma propriedade $X$ em $\Sigma^{*}$, dizemos que a matriz de pontuação $\gamma$ induz a propriedade $X$ em opt $\mathrm{A}_{\gamma}\left(\right.$ opt $\mathrm{N}_{\gamma}$, opt $\left.\mathrm{E}_{\gamma}\right)$. Se opt $\mathrm{A}_{\gamma}\left(\right.$ opt $\mathrm{N}_{\gamma}$, opt $\left.\mathrm{E}_{\gamma}\right)$ é uma métrica (pramétrica, semimétrica, etc) em $\Sigma^{*}$ também dizemos que $\gamma$ induz métrica (pramétrica, semimétrica, etc) na função opt $\mathrm{A}_{\gamma}\left(\right.$ opt $\mathrm{N}_{\gamma}$, opt $\left.\mathrm{E}_{\gamma}\right)$.

A classe $\mathbb{M}^{C}$ é uma classe comum de matrizes de pontuação que possui as seguintes propriedades para cada a, b, c, $\in \Sigma_{\sqcup}$ : 


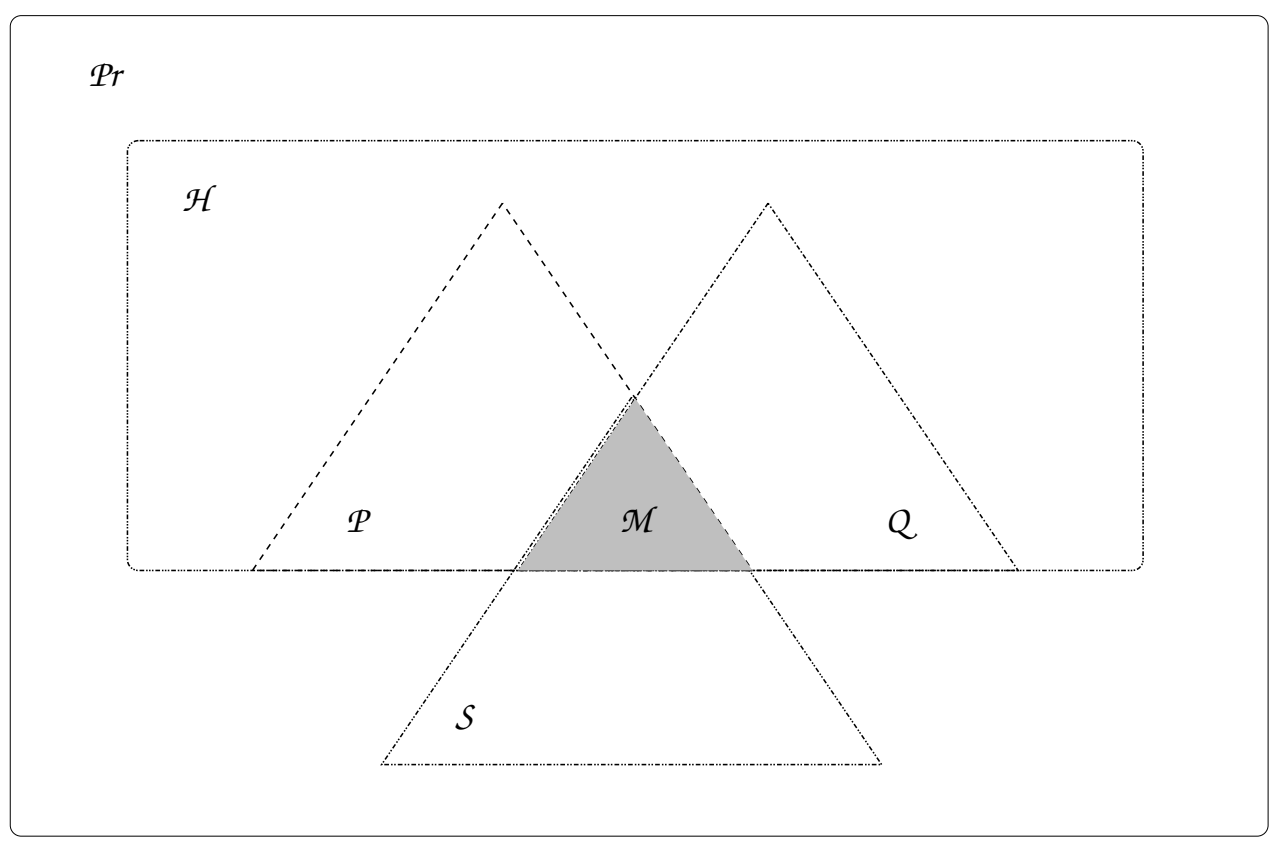

Figura 4.1: Conjunto das funções: a área sombreada representa o conjunto formado por métricas, que é a intersecção dos demais conjuntos de funçôes estudados.

1. $\gamma_{\mathrm{a} \rightarrow \mathrm{b}}>0$ se $\mathrm{a} \neq \mathrm{b}, \mathrm{e} \gamma_{\mathrm{a} \rightarrow \mathrm{b}}=0$ se $\mathrm{a}=\mathrm{b}$,

2. $\gamma_{\mathrm{a} \rightarrow \mathrm{b}}=\gamma_{\mathrm{b} \rightarrow \mathrm{a}}$,

3. $\gamma_{\mathrm{a} \rightarrow \mathrm{c}} \leq \gamma_{\mathrm{a} \rightarrow \mathrm{b}}+\gamma_{\mathrm{b} \rightarrow \mathrm{c}}$.

Sellers [Sel74] mostrou que as matrizes em $\mathbb{M}^{\mathrm{C}}$ induzem métrica em opt $\mathrm{A}_{\gamma}$. No entanto, definimos $\mathbb{M}^{\mathbb{A}}$ que é a classe das matrizes de pontuação tais que, para cada $a, b, c \in \Sigma$, valem

1. $\gamma_{\mathrm{a} \rightarrow \sqcup}=\gamma_{\sqcup \rightarrow \mathrm{a}}>0$,

2. $\gamma_{\mathrm{a} \rightarrow \mathrm{b}}>0$ se $\mathrm{a} \neq \mathrm{b}$, e $\gamma_{\mathrm{a} \rightarrow \mathrm{b}}=0$ se $\mathrm{a}=\mathrm{b}$,

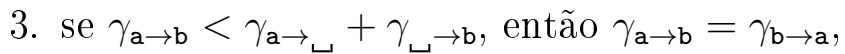

4. $\gamma_{\mathrm{a} \rightarrow \sqcup} \leq \gamma_{\mathrm{a} \rightarrow \mathrm{b}}+\gamma_{\mathrm{b} \rightarrow \sqcup}$,

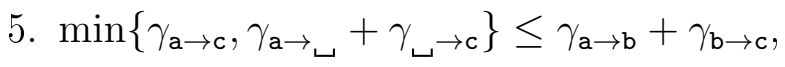

e mostramos neste capítulo que esta é a classe que contém exatamente todas as matrizes que induzem métrica em opt $\mathrm{A}_{\gamma}$, o que implica, dado que toda matriz em $\mathbb{M}^{\mathrm{C}}$ induz métrica em opt $\mathrm{A}_{\gamma}$, que $\mathbb{M}^{\mathrm{C}} \subseteq \mathbb{M}^{\mathrm{A}}$. Além disso, a matriz exibida em (1.1) no Capítulo 1, como vimos, pertence a $\mathbb{M}^{\mathrm{A}}$ e não pertence à $\mathbb{M}^{\mathrm{C}}$ o que implica que $\mathbb{M}^{\mathrm{C}} \neq \mathbb{M}^{\mathrm{A}}$.

Marzal e Vidal [MV93] observaram que nem toda matriz em $\mathbb{M}^{C}$ induz métrica em opt $\mathrm{N}_{\gamma}$. Eles mostraram, por exemplo, que a função opt $\mathrm{N}_{\gamma}$ induzida pela matriz

$$
\gamma=\begin{array}{c|ccc} 
& \mathrm{a} & \mathrm{b} & \mathrm{c} \\
\hline \mathrm{a} & 0 & 5 & 5 \\
\mathrm{~b} & 5 & 0 & 1 \\
- & 5 & 1 &
\end{array},
$$


que pertence a $\mathbb{M}^{\mathrm{C}}$, não respeita a propriedade da desigualdade triangular nas sequências desde que

$$
\begin{aligned}
\operatorname{opt} \mathrm{N}_{\gamma}(\mathrm{a}, \mathrm{b}) & =\min \left\{v \mathrm{~N}_{\gamma}\left(\left[\begin{array}{cc}
\mathrm{a} & \sqcup \\
& \mathrm{b}
\end{array}\right]\right), v \mathrm{~N}_{\gamma}\left(\left[\begin{array}{cc}
\sqcup & \mathrm{a} \\
\mathrm{b} & \sqcup
\end{array}\right]\right), v \mathrm{~N}_{\gamma}\left(\left[\begin{array}{l}
\mathrm{a} \\
\mathrm{b}
\end{array}\right]\right)\right\}=3 \\
& >\frac{1}{2}+\frac{7}{3}=v \mathrm{~N}_{\gamma}\left(\left[\begin{array}{cc}
\mathrm{a} & \sqcup \\
\mathrm{a} & \mathrm{b}
\end{array}\right]\right)+v \mathrm{~N}_{\gamma}\left(\left[\begin{array}{ccc}
\mathrm{a} & \mathrm{b} & \sqcup \\
\sqcup & \sqcup & \mathrm{b}
\end{array}\right]\right) \\
& \geq \operatorname{opt}_{\gamma}(\mathrm{a}, \mathrm{ab})+\operatorname{opt} \mathrm{N}_{\gamma}(\mathrm{ab}, \mathrm{b}) .
\end{aligned}
$$

Yujian e Bo [YB07] observaram que é um problema em aberto determinar se uma dada matriz de pontuação $\gamma$ induz ou não métrica em opt $\mathrm{N}_{\gamma}$.

Nós definimos a classe das matrizes $\mathbb{M}^{\mathbb{N}}$ que, como também mostramos neste capítulo, é exatamente a classe das matrizes $\gamma$ que induzem métrica em opt $\mathrm{N}_{\gamma}$. A classe $\mathbb{M}^{\mathbb{N}}$ possui as propriedades:

1. $\mathbb{M}^{\mathbb{N}} \subseteq \mathbb{M}^{\mathrm{A}} \mathrm{e}$

2. $\gamma_{\mathrm{a} \rightarrow \sqcup} \leq 2 \gamma_{\mathrm{b} \rightarrow \sqcup}$ para cada $\mathrm{a}, \mathrm{b} \in \Sigma$.

Note que a matriz em (1.1) do Capítulo 1 pertence à classe $\mathbb{M}^{\mathbb{N}}$ mas não pertence à classe $\mathbb{M}^{\mathrm{C}}$ e que a matriz em (4.1) não pertence à classe $\mathbb{M}^{\mathbb{N}}$ mas pertence à classe $\mathbb{M}^{\mathrm{C}}$. Logo, $\mathbb{M}^{\mathbb{N}} \nsubseteq \mathbb{M}^{\mathrm{C}}$ e $\mathbb{M}^{\mathrm{C}} \nsubseteq \mathbb{M}^{\mathbb{N}}$.

Outra classe de matrizes de pontuação que estudamos é a classe $\mathbb{M}^{\mathrm{E}}$. Ela possui as seguintes propriedades:

1. $\gamma_{\mathrm{a} \rightarrow \mathrm{a}} \geq 0$ para cada $\mathrm{a} \in \Sigma$,

2. $\gamma_{\mathrm{a} \rightarrow \mathrm{b}}, \gamma_{\mathrm{a} \rightarrow}, \gamma_{\boldsymbol{\zeta} \rightarrow \mathrm{a}}>0$ para cada $\mathrm{a} \neq \mathrm{b}, \mathrm{a}, \mathrm{b} \in \Sigma$,

3. $\mathcal{P}_{\gamma}(\mathrm{a}, \sqcup)=\mathcal{P}_{\gamma}\left(\_, \mathrm{a}\right)$ e $\mathcal{P}_{\gamma}(\mathrm{a}, \mathrm{b})=\mathcal{P}_{\gamma}(\mathrm{b}, \mathrm{a})$ para todo $\mathrm{a} \neq \mathrm{b} \in \Sigma$, onde $\mathcal{P}_{\gamma}(x, y)$ denota o custo de um caminho ótimo do vértice $x$ ao vértice $y$ no digrafo $D(\gamma)$.

Mostramos também neste capítulo que $\mathbb{M}^{\mathrm{E}}$ é exatamente a classe das matrizes de pontuação que induzem métrica em opt $\mathrm{E}_{\gamma}$.

O fato a seguir tem como consequência que $\mathbb{M}^{E}$ abrange as demais classes de matrizes de pontuação aqui estudadas.

Fato 39. $\mathbb{M}^{\mathrm{A}} \subseteq \mathbb{M}^{\mathrm{E}}$.

Prova. Para um arco $e=u \rightarrow v$ no digrafo $D(\gamma)$, denotamos por $e^{-1}$ o arco $v \rightarrow u$ e, para um caminho $P=\left(e_{1}, \ldots, e_{n}\right)$ de um vértice a a um vértice b em $D(\gamma)$, denotamos por $P^{-1}$ o caminho $\left(e_{n}^{-1}, \ldots, e_{1}^{-1}\right)$ de b a a.

Seja $\gamma \in \mathbb{M}^{\mathbb{A}}$. Por definição de $\mathbb{M}^{\mathrm{A}}$ temos que $\gamma_{\mathrm{a} \rightarrow \mathrm{a}}=0, \gamma_{\mathrm{a} \rightarrow \mathrm{b}}, \gamma_{\mathrm{a} \rightarrow\lrcorner}, \gamma_{\llcorner\rightarrow \mathrm{a}}>0$ para cada $\mathrm{a} \neq \mathrm{b} \in \Sigma$. Logo, as Propriedades 1 e 2 da definição de $\mathbb{M}^{E}$ são satisfeitas e, para finalizar a prova, basta demonstrar que também vale a Propriedade 3 . Sejam a, b $\in \Sigma_{\llcorner}$ e $P=\left(e_{1}, \ldots, e_{n}\right)$ um caminho ótimo de a até b de maior comprimento em $D(\gamma)$. Logo, $\mathcal{P}_{\gamma}(\mathrm{a}, \mathrm{b})=\operatorname{custo}(P)=\sum_{i=1}^{n} \operatorname{custo}\left(e_{i}\right)$.

Seja $e$ um arco em $P$. Como $\_\rightarrow$ não é arco em $D(\gamma)$, o arco $e$ tem a forma $u \rightarrow \backsim$, $\sqcup \rightarrow v$ ou $u \rightarrow v \operatorname{com} u, v \in \Sigma$.

Se $e$ tem a forma $u \rightarrow \sqcup$, então, desde que $\gamma \in \mathbb{M}^{\mathrm{A}}$, temos que $\gamma_{u \rightarrow_{\sqcup}}=\gamma_{\varsigma^{\rightarrow} \rightarrow u}$ o que implica que custo $(e)=\gamma_{u \rightarrow \sqcup}=\gamma_{{ } \rightarrow u}=\operatorname{custo}\left(e^{-1}\right)$. Analogamente, se $e$ tem a forma $\rightarrow_{\sqcup} \rightarrow u$, então custo $(e)=\operatorname{custo}\left(e^{-1}\right)$. 


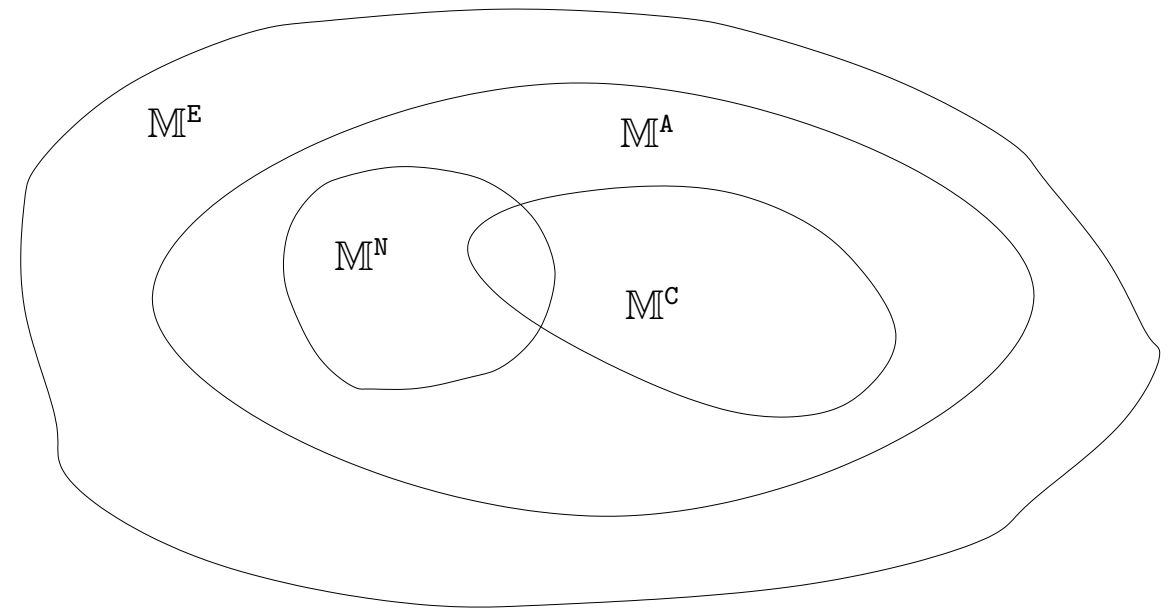

Figura 4.2: A figura mostra a relação entre as classes $\mathbb{M}^{\mathbb{E}}, \mathbb{M}^{\mathbb{A}}, \mathbb{M}^{\mathbb{N}}$ e $\mathbb{M}^{\mathrm{C}}$.

Se $e$ tem a forma $u \rightarrow v, u, v \in \Sigma$, então, sendo $P$ caminho ótimo de maior comprimento, custo $(u \rightarrow v)<$ custo $\left(u \rightarrow{ }_{\sqcup}\right)+$ custo $\left(_{\llcorner} \rightarrow v\right)$, o que implica que

$$
\left.\gamma_{u \rightarrow v}=\operatorname{custo}(u \rightarrow v)<\operatorname{custo}\left(u \rightarrow \rightarrow_{\sqcup}\right)+\operatorname{custo}_{(\iota} \rightarrow v\right)=\gamma_{u \rightarrow \sqcup}+\gamma_{\sqcup \rightarrow v} .
$$

Segue da definição de $\mathbb{M}^{\mathrm{A}}$ que $\gamma_{u \rightarrow v}=\gamma_{v \rightarrow u}$. Logo, custo $(e)=\gamma_{u \rightarrow v}=\gamma_{v \rightarrow u}=\operatorname{custo}\left(e^{-1}\right)$.

Dos três últimos parágrafos, concluímos que se e é arco de $P$, então custo $(e)=\operatorname{custo}\left(e^{-1}\right)$. Segue que custo $(P)=\sum_{i=1}^{n}$ custo $\left(e_{i}\right)=\sum_{i=1}^{n} \operatorname{custo}\left(e_{i}^{-1}\right)=\operatorname{custo}\left(P^{-1}\right)$, o que implica que $\mathcal{P}_{\gamma}(\mathrm{a}, \mathrm{b})=\operatorname{custo}(P)=\operatorname{custo}\left(P^{-1}\right) \geq \mathcal{P}_{\gamma}(\mathrm{b}, \mathrm{a})$. Desde que o resultado vale para quaisquer $\mathrm{a}, \mathrm{b} \in \Sigma_{\omega}$, segue que $\mathcal{P}_{\gamma}(\mathrm{b}, \mathrm{a}) \geq \mathcal{P}_{\gamma}(\mathrm{a}, \mathrm{b})$, o que nos permite concluir que $\mathcal{P}_{\gamma}(\mathrm{a}, \mathrm{b})=\mathcal{P}_{\gamma}(\mathrm{b}, \mathrm{a})$. Portanto, vale a Propriedade 3 da definição de $\mathbb{M}^{\mathrm{E}}$.

Das observações acima e do Fato 39 temos $\mathbb{M}^{\mathrm{C}} \subseteq \mathbb{M}^{\mathrm{A}} \subseteq \mathbb{M}^{\mathrm{E}}, \mathbb{M}^{\mathbb{N}} \subseteq \mathbb{M}^{\mathrm{A}}, \mathbb{M}^{\mathrm{C}} \nsubseteq \mathbb{M}^{\mathbb{N}}$ e $\mathbb{M}^{\mathbb{N}} \nsubseteq \mathbb{M}^{\mathrm{C}}$. A relação entre os conjuntos é mostrada na Figura 4.2.

Ainda vale observar que, para a relação dos conjuntos:

$$
\begin{array}{c|ccc} 
& \mathrm{a} & \mathrm{b} & \\
\hline \mathrm{a} & 0 & 1 & 1 \\
\mathrm{~b} & 1 & 0 & 1 \\
& 1 & 1 &
\end{array} \in \mathbb{M}^{\mathrm{C}} \cap \mathbb{M}^{\mathrm{N}}
$$

o que implica que $\mathbb{M}^{\mathrm{C}} \cap \mathbb{M}^{\mathbb{N}} \neq \emptyset$;

$$
\begin{array}{c|ccc} 
& \mathrm{a} & \mathrm{b} & \multicolumn{1}{c}{} \\
\hline \mathrm{a} & 0 & 3 & 3 \\
\mathrm{~b} & 3 & 0 & 1 \\
\sqcup & 3 & 1 &
\end{array} \in \mathbb{M}^{\mathrm{A}} \backslash\left(\mathbb{M}^{\mathrm{N}} \cup \mathbb{M}^{\mathrm{C}}\right)
$$

o que implica que $\mathbb{M}^{\mathrm{A}} \neq \mathbb{M}^{\mathbb{N}} \cup \mathbb{M}^{\mathrm{C}}$; 


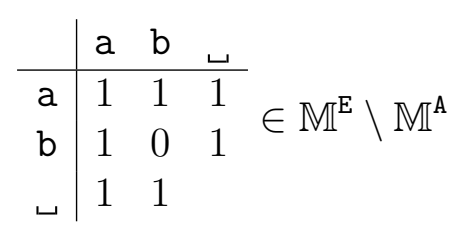

o que implica que $\mathbb{M}^{\mathrm{E}} \neq \mathbb{M}^{\mathrm{A}}$.

\subsection{Distância de alinhamento}

Nesta seção provamos cada uma das propriedades métricas de $\gamma$ em opt $\mathrm{A}_{\gamma}$.

Fato 40. Seja $\gamma$ uma matriz de pontuação. Se $D(\gamma)$ possui um ciclo negativo, então existe $s \in \Sigma^{*}$ tal que optA $\mathrm{A}_{\gamma}(s, s)<0$.

Prova. Suponha que $\mathrm{a}_{1}, \ldots, \mathrm{a}_{m} \in \Sigma$ são tais que $C=\left(\mathrm{a}_{1} \rightarrow \mathrm{a}_{2}, \ldots, \mathrm{a}_{m} \rightarrow \mathrm{a}_{1}\right)$ é um ciclo em $D(\gamma)$ e custo $(C)=-X<0$. Desde que ciclos com zero arcos possuem custo zero, segue que $C$ possui pelo menos um arco. Desde que $C$ possui pelo menos um arco $e_{\sqcup} \rightarrow \_$não é arco em $D(\gamma)$, segue que pelo menos um símbolo em $\mathrm{a}_{1}, \ldots, \mathrm{a}_{m}$ não é ${ }$. Sem perda de generalidade, assumimos que $\mathrm{a}_{1} \neq \omega_{\sqcup}$. Sejam um número inteiro $n>\left(\gamma_{\mathrm{a}_{1} \rightarrow \iota}+\gamma_{\iota \rightarrow \mathrm{a}_{1}}\right) / X$ e $s \in \Sigma^{*}$ a sequência obtida removendo os símbolos iguais $\mathrm{a}_{\sqcup}$ de $\left(\mathrm{a}_{1} \mathrm{a}_{2} \ldots \mathrm{a}_{m-1} \mathrm{a}_{m}\right)^{n} \mathrm{a}_{1}$. Logo, [ $\left.\left(\mathrm{a}_{1} \mathrm{a}_{2} \ldots \mathrm{a}_{m-1} \mathrm{a}_{m}\right)^{n} \mathrm{a}_{1}, \mathrm{a}_{1}\left(\mathrm{a}_{2} \ldots \mathrm{a}_{m-1} \mathrm{a}_{m} \mathrm{a}_{1}\right)^{n}{ }_{\longleftarrow}\right]$ é um alinhamento de $(s, s)$ e, portanto,

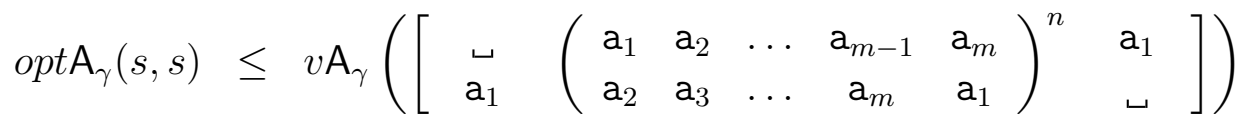

$$
\begin{aligned}
& =\gamma_{\iota \rightarrow a}-n X+\gamma_{\mathrm{a} \rightarrow \text { }} \\
& <\gamma_{\lrcorner \rightarrow a}-\left(\gamma_{a \rightarrow \sqcup}+\gamma_{\lrcorner \rightarrow a}\right)+\gamma_{a \rightarrow \sqcup}=0 \text {. }
\end{aligned}
$$

Portanto, se $D(\gamma)$ possui um ciclo negativo, então $\operatorname{opt}_{\gamma}(s, s)<0$ para algum $s \in \Sigma^{*}$.

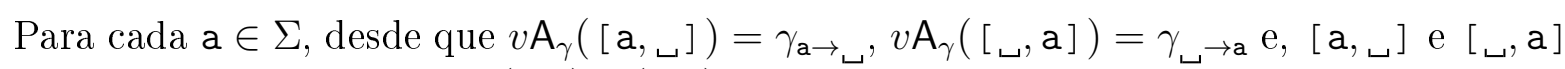
são os únicos alinhamentos de $(\mathrm{a}, \epsilon)$ e $(\epsilon, \mathrm{a})$ respectivamente, nós temos que

$$
\begin{aligned}
& \operatorname{opt}_{\gamma}(\mathrm{a}, \epsilon)=v \mathrm{~A}_{\gamma}([\mathrm{a},{ }])=\gamma_{\mathrm{a} \rightarrow \sqcup} \\
& \operatorname{opt}_{\gamma}(\epsilon, \mathrm{a})=v \mathrm{~A}_{\gamma}([\sqcup, \mathrm{a}])=\gamma_{\sqcup \rightarrow \mathrm{a}}
\end{aligned}
$$

Para cada $\mathrm{a}, \mathrm{b} \in \Sigma$, desde que

$$
\mathcal{A}_{\{(\mathrm{a}, \mathrm{b})\}}=\left\{\left[\begin{array}{l}
\mathrm{a} \\
\mathrm{b}
\end{array}\right],\left[\begin{array}{cc}
\mathrm{a} & \sqcup \\
& \mathrm{b}
\end{array}\right],\left[\begin{array}{cc}
\sqcup & \mathrm{a} \\
\mathrm{b} & \sqcup
\end{array}\right]\right\},
$$

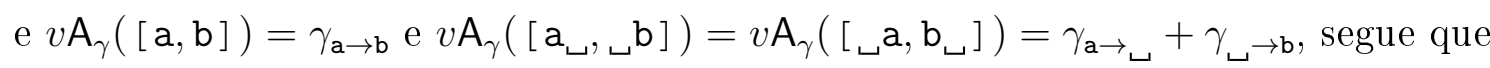

$$
\operatorname{opt}_{\gamma}(\mathrm{a}, \mathrm{b})=\min \left\{\begin{array}{l}
v \mathrm{~A}_{\gamma}([\mathrm{a}, \mathrm{b}])=\gamma_{\mathrm{a} \rightarrow \mathrm{b}} \\
v \mathrm{~A}_{\gamma}\left(\left[\mathrm{a}_{\sqcup}, \mathrm{b}\right]\right)=\gamma_{\mathrm{a} \rightarrow \sqcup}+\gamma_{\lrcorner \rightarrow \mathrm{b}} .
\end{array}\right.
$$

Lema 41. Seja $\gamma$ uma matriz de pontuação. Então, opt $\mathrm{A}_{\gamma}(s, s)=0$ para cada $s \in \Sigma^{*}$ se e somente se

(i) $D(\gamma)$ não possui ciclo negativo e 


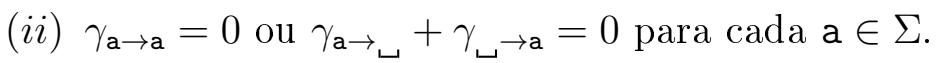

Prova. Seja a $\in \Sigma$ e suponha que $\operatorname{optA}_{\gamma}(s, s)=0$ para cada $s \in \Sigma^{*}$. Logo, pelo Fato 40 vale $(i)$ e como optA $(\mathrm{a}, \mathrm{a})=0$, usando (4.4), temos que $\gamma_{\mathrm{a} \rightarrow \mathrm{a}}=0$ ou $\gamma_{\mathrm{a} \rightarrow \sqcup}+\gamma_{\lrcorner \rightarrow \mathrm{a}}=0$, o que implica que vale $(i i)$. Portanto, se $\operatorname{optA}_{\gamma}(s, s)=0$ para cada $s \in \Sigma^{*}$, então valem $(i)$ e $(i i)$.

Reciprocamente, suponha que valem $(i)$ e $(i i)$. Sejam $s=s(1) s(2) \ldots s(n) \in \Sigma^{*}$ e $A$ um alinhamento A-ótimo de $(s, s)$. Definimos um digrafo $H$ para o alinhamento $A$, tal que

$$
\begin{aligned}
& V(H)=\{1,2, \ldots, n, \sqcup\}, \\
& E(H)=\{i \rightarrow j \mid \text { o } i \text {-ésimo e o } j \text {-ésimo símbolo de } s \text { estão alinhados em } A\} \cup \\
& \{i \rightarrow \sim \mid \text { o } i \text {-ésimo símbolo de } s \text { e } \sqcup \text { estão alinhados em } A\} \cup \\
& \left\{\_\rightarrow j \mid \sqcup \text { e o o } j \text {-ésimo símbolo de } s \text { estão alinhados em } A\right\} \text {, }
\end{aligned}
$$

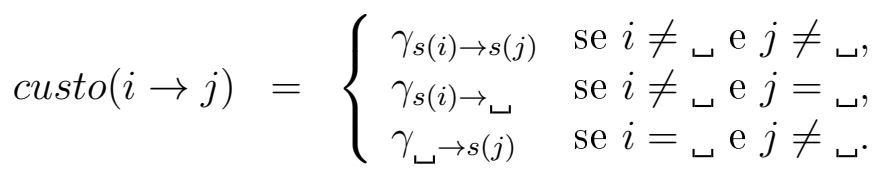

Por construção, temos que $v \mathrm{~A}_{\gamma}(A)=\operatorname{custo}(H)$. Temos também que $H$ é euleriano e, portanto, pelo Teorema 2, $E(H)$ pode ser decomposto em ciclos disjuntos nos arcos. Para cada ciclo dessa decomposição, existe um ciclo correspondente e de mesmo custo em $D(\gamma)$. Desde que, por hipótese, $D(\gamma)$ não possui ciclo de custo negativo, segue que custo $(H) \geq 0$. Segue que

$$
\operatorname{opt}_{\gamma}(s, s)=v \mathrm{~A}_{\gamma}(A)=\operatorname{custo}(H) \geq 0 .
$$

Além disso, construímos o seguinte alinhamento $B$ : alinhe $[s(i), s(i)]$ se $\gamma_{s(i) \rightarrow s(i)}=0$ e alinhe $\left[s(i),_{\sqcup}\right]$ e $[\sqcup, s(i)]$ caso contrário. Desde que vale $(i i)$, segue que $v \mathrm{~A}_{\gamma}(B)=0$. Então,

$$
\operatorname{opt}_{\gamma}(s, s) \leq v \mathrm{~A}_{\gamma}(B)=0
$$

Usando (4.5) e (4.6), e desde que o argumento é usado para qualquer $s \in \Sigma^{*}$, concluímos que se valem $(i)$ e $(i i)$, então $\operatorname{opt} \mathrm{A}_{\gamma}(s, s)=0$ para cada $s \in \Sigma^{*}$.

Lema 42. Seja $\gamma$ uma matriz de pontuação. Então, optA $(s, t) \geq 0$ para cada $s, t \in \Sigma^{*}$ se e somente se $\gamma_{\mathrm{a} \rightarrow \boldsymbol{}}, \gamma_{\boldsymbol{L}} \rightarrow \mathrm{b}, \gamma_{\mathrm{a} \rightarrow \mathrm{b}} \geq 0$ para cada $\mathrm{a}, \mathrm{b} \in \Sigma$.

Prova. Suponha que $\operatorname{optA}_{\gamma}(s, t) \geq 0$ para cada $s, t \in \Sigma^{*}$. Segue de (4.2), (4.3) e (4.4) que

$$
\begin{aligned}
& \gamma_{\mathrm{a} \rightarrow \sqcup}=v \mathrm{~A}_{\gamma}([\mathrm{a}, \sqcup])=\operatorname{opt} \mathrm{A}_{\gamma}(\mathrm{a}, \epsilon) \geq 0 \\
& \gamma_{\sqcup \rightarrow \mathrm{b}}=v \mathrm{~A}_{\gamma}([\sqcup, \mathrm{b}])=\operatorname{opt} \mathrm{A}_{\gamma}(\epsilon, \mathrm{b}) \geq 0 \\
& \gamma_{\mathrm{a} \rightarrow \mathrm{b}}=v \mathrm{~A}_{\gamma}([\mathrm{a}, \mathrm{b}]) \geq \operatorname{opt} \mathrm{A}_{\gamma}(\mathrm{a}, \mathrm{b}) \geq 0
\end{aligned}
$$

para cada $\mathrm{a}, \mathrm{b} \in \Sigma$. Logo, se opt $\mathrm{A}_{\gamma}(s, t) \geq 0$ para cada $s, t \in \Sigma^{*}$, então $\gamma_{\mathrm{a} \rightarrow \sqcup}, \gamma_{\llcorner} \rightarrow \mathrm{b}, \gamma_{\mathrm{a} \rightarrow \mathrm{b}} \geq 0$ para cada $\mathrm{a}, \mathrm{b} \in \Sigma$.

Reciprocamente, sejam $s, t \in \Sigma^{*}$ e suponha que $\gamma_{\mathrm{a} \rightarrow\lrcorner}, \gamma_{\lrcorner \rightarrow \mathrm{b}}, \gamma_{\mathrm{a} \rightarrow \mathrm{b}} \geq 0$ para cada $\mathrm{a}, \mathrm{b} \in \Sigma$, e que $A$ é um alinhamento A-ótimo de $(s, t)$. Desde que $v \mathrm{~A}_{\gamma}(A)$ é a soma das entradas de $\gamma$ que são maiores ou iguais a zero, segue que opt $\mathrm{A}_{\gamma}(s, t)=v \mathrm{~A}_{\gamma}(A) \geq 0$. Portanto, se $\gamma_{\mathrm{a} \rightarrow \boldsymbol{}}, \gamma_{\boldsymbol{L}_{\rightarrow}}, \gamma_{\mathrm{a} \rightarrow \mathrm{b}} \geq 0$ para cada $\mathrm{a}, \mathrm{b} \in \Sigma$, então $\operatorname{optA}_{\gamma}(s, t) \geq 0$ para cada $s, t \in \Sigma^{*}$. 
Lema 43. Seja $\gamma$ uma matriz de pontuação. Então, opt $\mathrm{A}_{\gamma}(s, t)>0$ para cada $s \neq t \in \Sigma^{*}$ se e somente se, para cada $\mathrm{a} \neq \mathrm{b} \in \Sigma$, valem

(i) $\gamma_{\mathrm{a} \rightarrow \mathrm{a}} \geq 0$

(ii) $\gamma_{\mathrm{a} \rightarrow \mathrm{b}}, \gamma_{\mathrm{a} \rightarrow \sqcup}, \gamma_{\lrcorner \rightarrow \mathrm{b}}>0$.

Prova. Sejam $\mathrm{a} \neq \mathrm{b} \in \Sigma$ e suponha que $\operatorname{opt}_{\gamma}(s, t)>0$ para cada $s \neq t \in \Sigma^{*}$. Desde que, para um inteiro arbitrário $n \geq 0$, $\left[\mathrm{a}^{n+1}, \mathrm{a}^{n}{ }\right]$ é um alinhamento de $\left(\mathrm{a}^{n+1}, \mathrm{a}^{n}\right)$ e, por hipótese, opt $\mathrm{A}_{\gamma}\left(\mathrm{a}^{n+1}, \mathrm{a}^{n}\right)>0$, segue que

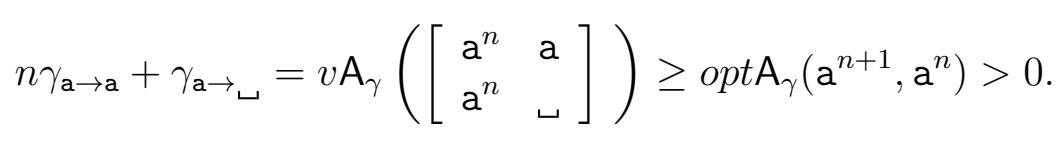

Desde que a expressão acima vale para um inteiro arbitrário não negativo $n$, temos que $\gamma_{\mathrm{a} \rightarrow \mathrm{a}} \geq 0$. Um argumento similar usado no primeiro parágrafo da prova do Lema 42 mostra que $\gamma_{\mathrm{a} \rightarrow \mathrm{b}}, \gamma_{\mathrm{a} \rightarrow \boldsymbol{b}}, \gamma_{\mathrm{u} \rightarrow \mathrm{b}}>0$. Desde que os argumentos valem para quaisquer $\mathrm{a} \neq \mathrm{b} \in \Sigma$, segue que se $\operatorname{optA}_{\gamma}(s, t)>0$ para cada $s \neq t \in \Sigma^{*}$, então valem $(i)$ e $(i i)$.

Reciprocamente, seja $s \neq t \in \Sigma^{*}$ e suponha que valem $(i)$ e $(i i)$ e que $\left[s^{\prime}, t^{\prime}\right]$ é um alinhamento de $(s, t)$. Desde que $s \neq t$, existe $h$ tal que $s^{\prime}(h) \neq t^{\prime}(h)$, o que implica por $(i i)$ que $\gamma_{s^{\prime}(h) \rightarrow t^{\prime}(h)}>0$. Desde que, por $(i)$ e $(i i), \gamma_{s^{\prime}(j) \rightarrow t^{\prime}(j)} \geq 0$ para cada $j \neq h$, segue que

$$
\operatorname{opt}_{\gamma}(s, t) \geq v \mathrm{~A}_{\gamma}\left(\left[s^{\prime}, t^{\prime}\right]\right)=\gamma_{s^{\prime}(h) \rightarrow t^{\prime}(h)}+\sum_{j \neq h} \gamma_{s^{\prime}(j) \rightarrow t^{\prime}(j)} \geq \gamma_{s^{\prime}(h) \rightarrow t^{\prime}(h)}+0=\gamma_{s^{\prime}(h) \rightarrow t^{\prime}(h)}>0 .
$$

Portanto, se valem $(i)$ e $(i i)$, então $o p t \mathrm{~A}_{\gamma}(s, t)>0$ para cada $s \neq t \in \Sigma^{*}$.

Lema 44. Seja $\gamma$ uma matriz de pontuação. Então, opt $\mathrm{A}_{\gamma}(s, t)=\operatorname{opt} \mathrm{A}_{\gamma}(t, s)$ para cada $s, t \in \Sigma^{*}$ se e somente valem, para cada a, $\mathrm{b} \in \Sigma$,

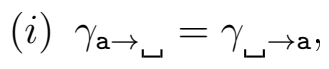

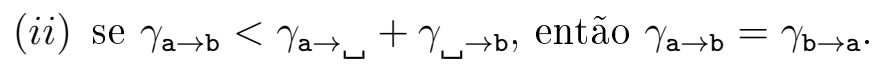

Prova. Sejam $\mathrm{a}, \mathrm{b} \in \Sigma$ e suponha que opt $\mathrm{A}_{\gamma}(s, t)=\operatorname{opt} \mathrm{A}_{\gamma}(t, s)$ para cada $s, t \in \Sigma^{*}$. Segue como consequência de (4.2) e (4.3) que

$$
\gamma_{\mathrm{a} \rightarrow \boldsymbol{\sqcup}}=\operatorname{optA}_{\gamma}(\mathrm{a}, \epsilon)=\operatorname{optA}_{\gamma}(\epsilon, \mathrm{a})=\gamma_{\boldsymbol{\iota} \rightarrow \mathrm{a}} .
$$

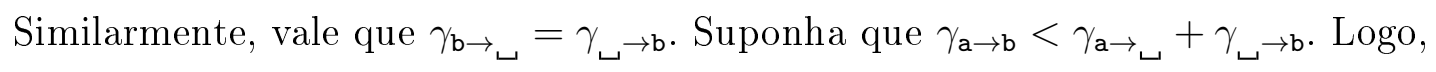

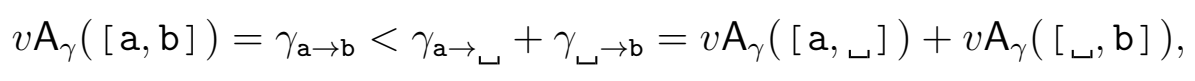

o que implica por (4.4) que [a,b] é A-ótimo. Desde que optA $(\mathrm{b}, \mathrm{a})=\operatorname{optA}_{\gamma}(\mathrm{a}, \mathrm{b})$, segue que

$$
\begin{aligned}
\operatorname{opt}_{\gamma}(\mathrm{b}, \mathrm{a})=\operatorname{optA}_{\gamma}(\mathrm{a}, \mathrm{b})=v \mathrm{~A}_{\gamma}([\mathrm{a}, \mathrm{b}]) & =\gamma_{\mathrm{a} \rightarrow \mathrm{b}} \\
& <\gamma_{\mathrm{a} \rightarrow \sqcup}+\gamma_{\sqcup \rightarrow \mathrm{b}}=\gamma_{\mathrm{b} \rightarrow \sqcup}+\gamma_{\sqcup \rightarrow \mathrm{a}} .
\end{aligned}
$$

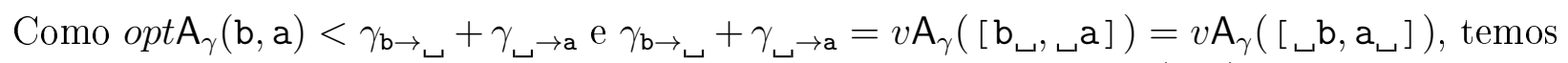
que nem $\left[b_{\sqcup},{ }_{\sqcup} a\right]$ e nem $\left[\sqcup, a_{\sqcup}\right]$ são alinhamentos A-ótimos de $(b, a)$. Segue novamente de (4.4) que

$$
\operatorname{opt}_{\gamma}(\mathrm{b}, \mathrm{a})=v \mathrm{~A}_{\gamma}([\mathrm{b}, \mathrm{a}])=\gamma_{\mathrm{b} \rightarrow \mathrm{a}}
$$


Usando (4.7) e (4.8), concluímos que $\gamma_{\mathrm{a} \rightarrow \mathrm{b}}=\operatorname{opt}_{\gamma}(\mathrm{b}, \mathrm{a})=\gamma_{\mathrm{b} \rightarrow \mathrm{a}}$. Portanto, se opt $\mathrm{A}_{\gamma}(s, t)=$ opt $\mathrm{A}_{\gamma}(t, s)$ para cada $s, t \in \Sigma^{*}$, então valem $(i)$ e $(i i)$ para cada a, $\mathrm{b} \in \Sigma$.

Reciprocamente, sejam $s, t \in \Sigma^{*}$ e considere que valem $(i)$ e $(i i)$ para cada a, b $\in \Sigma$. Suponha que $\left[s^{\prime}, t^{\prime}\right]$ é um alinhamento A-ótimo de $(s, t)$ de comprimento máximo e $j$ é um inteiro tal que $1 \leq j \leq\left|\left[s^{\prime}, t^{\prime}\right]\right|$. Como $\left[s^{\prime}, t^{\prime}\right]$ é um alinhamento, temos que $s^{\prime}(j) \neq \sqcup$ ou $t^{\prime}(j) \neq \omega_{\omega}$. Logo, se $s^{\prime}(j)=_{\leftarrow}$, então $t^{\prime}(j) \neq \neq_{\omega}$, o que implica, por $(i)$, que $\gamma_{s^{\prime}(j) \rightarrow t^{\prime}(j)}=$ $\gamma_{t^{\prime}(j) \rightarrow s^{\prime}(j)}$. Similarmente se $t^{\prime}(j)=\iota$, então $\gamma_{s^{\prime}(j) \rightarrow t^{\prime}(j)}=\gamma_{t^{\prime}(j) \rightarrow s^{\prime}(j)}$. Para finalizar a prova, resta verificar o caso $s^{\prime}(j) \neq \_$e $t^{\prime}(j) \neq \_$. Desde que $\left[s^{\prime}, t^{\prime}\right]$ tem comprimento máximo, temos que

$$
\begin{array}{r}
\gamma_{s^{\prime}(j) \rightarrow t^{\prime}(j)}+\sum_{p \neq j} \gamma_{s^{\prime}(p) \rightarrow t^{\prime}(p)}=v \mathrm{~A}_{\gamma}\left(\left[s^{\prime}, t^{\prime}\right]\right)< \\
v \mathrm{~A}_{\gamma}\left(\left[\begin{array}{ccc|c}
s^{\prime}(1 \ldots j-1) & s^{\prime}(j) & \sqcup & s^{\prime}\left(j+1 \ldots\left|\left(s^{\prime}, t^{\prime}\right)\right|\right) \\
t^{\prime}(1 \ldots j-1) & \sqcup & t^{\prime}(j) & t^{\prime}\left(j+1 \ldots\left|\left(s^{\prime}, t^{\prime}\right)\right|\right)
\end{array}\right]\right)= \\
\\
\gamma_{s^{\prime}(j) \rightarrow \sqcup}+\gamma_{\sqcup \rightarrow t^{\prime}(j)}+\sum_{p \neq j} \gamma_{s^{\prime}(p) \rightarrow t^{\prime}(p)},
\end{array}
$$

o que implica que $\gamma_{s^{\prime}(j) \rightarrow t^{\prime}(j)}<\gamma_{s^{\prime}(j) \rightarrow_{\sqcup}}+\gamma_{\iota^{\prime} \rightarrow t^{\prime}(j)}$. Segue de $(i i)$ que, também nesse caso, $\gamma_{s^{\prime}(j) \rightarrow t^{\prime}(j)}=\gamma_{t^{\prime}(j) \rightarrow s^{\prime}(j)}$. Como $\gamma_{s^{\prime}(j) \rightarrow t^{\prime}(j)}=\gamma_{t^{\prime}(j) \rightarrow s^{\prime}(j)}$ para cada $j$, temos que $v \mathrm{~A}_{\gamma}\left(\left[s^{\prime}, t^{\prime}\right]\right)=$ $v \mathrm{~A}_{\gamma}\left(\left[t^{\prime}, s^{\prime}\right]\right)$. Desde que $\left[s^{\prime}, t^{\prime}\right]$ é um alinhamento A-ótimo de $(s, t)$ e $\left[t^{\prime}, s^{\prime}\right]$ é um alinhamento de $(t, s)$, segue que

$$
\operatorname{opt} \mathrm{A}_{\gamma}(s, t)=v \mathrm{~A}_{\gamma}\left(\left[s^{\prime}, t^{\prime}\right]\right)=v \mathrm{~A}_{\gamma}\left(\left[t^{\prime}, s^{\prime}\right]\right) \geq \operatorname{opt} \mathrm{A}_{\gamma}(t, s) .
$$

Usando os mesmos argumentos podemos mostrar que opt $\mathrm{A}_{\gamma}(t, s) \geq$ opt $\mathrm{A}_{\gamma}(s, t)$, o que nos permite concluir que opt $\mathrm{A}_{\gamma}(s, t)=o p t \mathrm{~A}_{\gamma}(t, s)$. Portanto, se valem $(i)$ e $(i i)$ para cada $\mathrm{a}, \mathrm{b} \in \Sigma$, então opt $\mathrm{A}_{\gamma}(s, t)=$ opt $\mathrm{A}_{\gamma}(t, s)$ para cada $s, t \in \Sigma^{*}$.

Proposição 45. Sejam $\gamma$ uma matriz de pontuação, $\lambda$ um número inteiro e $s, t, u \in \Sigma^{*}$. Se

(i) $\gamma_{\mathrm{a} \rightarrow \sqcup} \leq \gamma_{\mathrm{a} \rightarrow \mathrm{b}}+\gamma_{\mathrm{b} \rightarrow \sqcup}$,

(ii) $\gamma_{\mathrm{S} \rightarrow \mathrm{a}} \leq \gamma_{\mathrm{S} \rightarrow \mathrm{b}}+\gamma_{\mathrm{b} \rightarrow \mathrm{a}}$,

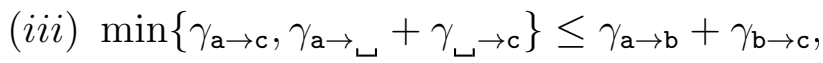

(iv) $\gamma_{\mathrm{b} \rightarrow \sqcup}+\gamma_{\sqcup \rightarrow \mathrm{b}} \geq \lambda$,

para cada a, b, c $\in \Sigma$, então, para cada alinhamento $A$ de $(s, t)$ e cada alinhamento $B$ de $(t, u)$, existem um alinhamento $C$ de $(s, u)$ e um inteiro $k \geq 0$ tais que

$$
v \mathrm{~A}_{\gamma}(A)+v \mathrm{~A}_{\gamma}(B) \geq v \mathrm{~A}_{\gamma}(C)+k \lambda, \quad|A| \leq|C|+k \text { e }|B| \leq|C|+k .
$$

Prova. Suponha que valem os itens $(i),(i i),(i i i),(i v)$ para cada a, b, c $\in \Sigma$ e que $A, B$ são 
alinhamentos de $(s, t)$ e $(t, u)$ respectivamente. Definimos

$$
\begin{aligned}
& \mathcal{C}_{1}=\left\{h \mid\left[s(h),{ }_{\longleftarrow}\right] \text { alinha em } A\right\}, \\
& \mathcal{C}_{2}=\{k \mid[{ }, u(k)] \text { alinha em } B\} \text {, } \\
& \mathcal{C}_{3}=\left\{j \mid\left[{ }_{\sqcup}, t(j)\right] \text { alinha em } A \text { e }\left[t(j),{ }_{\sqcup}\right] \text { alinha em } B\right\}, \\
& \mathcal{C}_{4}=\left\{(h, j) \mid[s(h), t(j)] \text { alinha em } A \text { e }\left[t(j),{ }_{\sqcup}\right] \text { alinha em } B\right\}, \\
& \mathcal{C}_{5}=\left\{(j, k) \mid\left[{ }_{\sqcup}, t(j)\right] \text { alinha em } A \text { e }[t(j), u(k)] \text { alinha em } B\right\}, \\
& \mathcal{C}_{6}=\left\{\begin{array}{l}
(h, j, k) \mid[s(h), t(j)] \text { alinha em } A,[t(j), u(k)] \text { alinha em } B \\
\mathrm{e} \gamma_{s(h) \rightarrow u(k)} \leq \gamma_{s(h) \rightarrow t(j)}+\gamma_{t(j) \rightarrow u(k)}
\end{array}\right\}, \\
& \mathcal{C}_{7}=\left\{\begin{array}{l}
(h, j, k) \mid[s(h), t(j)] \text { alinha em } A,[t(j), u(k)] \text { alinha em } B \\
\text { e } \gamma_{s(h) \rightarrow u(k)}>\gamma_{s(h) \rightarrow t(j)}+\gamma_{t(j) \rightarrow u(k)}
\end{array}\right\} .
\end{aligned}
$$

Então,

$$
\begin{aligned}
v \mathrm{~A}_{\gamma}(A)= & \sum_{h \in \mathcal{C}_{1}} \gamma_{s(h) \rightarrow \sqcup}+\sum_{j \in \mathcal{C}_{3}} \gamma_{\sqcup \rightarrow t(j)}+\sum_{(h, j) \in \mathcal{C}_{4}} \gamma_{s(h) \rightarrow t(j)}+ \\
& \sum_{(j, k) \in \mathcal{C}_{5}} \gamma_{\sqcup \rightarrow t(j)}+\sum_{(h, j, k) \in \mathcal{C}_{6}} \gamma_{s(h) \rightarrow t(j)}+ \\
& \sum_{(h, j, k) \in \mathcal{C}_{7}} \gamma_{s(h) \rightarrow t(j)}
\end{aligned}
$$

e

$$
\begin{aligned}
v \mathrm{~A}_{\gamma}(B)= & \sum_{k \in \mathcal{C}_{2}} \gamma_{\sqcup \rightarrow u(k)}+\sum_{j \in \mathcal{C}_{3}} \gamma_{t(j) \rightarrow}+\sum_{(h, j) \in \mathcal{C}_{4}} \gamma_{t(j) \rightarrow \sqcup}+ \\
& \sum_{(j, k) \in \mathcal{C}_{5}} \gamma_{t(j) \rightarrow u(k)}+\sum_{(h, j, k) \in \mathcal{C}_{6}} \gamma_{t(j) \rightarrow u(k)}+ \\
& \sum_{(h, j, k) \in \mathcal{C}_{7}} \gamma_{t(j) \rightarrow u(k)} .
\end{aligned}
$$

Definimos a seguir o alinhamento $C$ de $(s, u)$ de acordo com as seguintes três regras. Se $(h, j, k) \in \mathcal{C}_{6}$, então alinhe $[s(h), u(k)]$. Para cada $s(h)$ ainda não alinhado, alinhe $\left[s(h),{ }_{\longleftarrow}\right]$. Para cada $u(k)$ ainda não alinhado, alinhe $[\sqcup, u(k)]$. Portanto,

$$
\begin{aligned}
v \mathrm{~A}_{\gamma}(C)= & \sum_{h \in \mathcal{C}_{1}} \gamma_{s(h) \rightarrow \sqcup}+\sum_{k \in \mathcal{C}_{2}} \gamma_{\sqcup \rightarrow u(k)}+\sum_{(h, j) \in \mathcal{C}_{4}} \gamma_{s(h) \rightarrow \sqcup}+ \\
& \sum_{(j, k) \in \mathcal{C}_{5}} \gamma_{\sqcup \rightarrow u(k)}+\sum_{(h, j, k) \in \mathcal{C}_{6}} \gamma_{s(h) \rightarrow u(k)}+ \\
& \sum_{(h, j, k) \in \mathcal{C}_{7}}\left(\gamma_{s(h) \rightarrow \sqcup}+\gamma_{\sqcup \rightarrow u(k)}\right) .
\end{aligned}
$$

Se $j \in \mathcal{C}_{3}$, então, desde que vale $(i v)$, nós temos

$$
\begin{aligned}
\sum_{j \in \mathcal{C}_{3}} \gamma_{\sqcup \rightarrow t(j)}+\sum_{j \in \mathcal{C}_{3}} \gamma_{t(j) \rightarrow \sqcup} & =\sum_{j \in \mathcal{C}_{3}}\left(\gamma_{\sqcup \rightarrow t(j)}+\gamma_{t(j) \rightarrow}\right) \\
& \geq \sum_{j \in \mathcal{C}_{3}} \lambda=\left|\mathcal{C}_{3}\right| \lambda .
\end{aligned}
$$


Por $(i)$, (ii) e pela definição de $\mathcal{C}_{6}$, temos, respectivamente,

$$
\begin{aligned}
\sum_{(h, j) \in \mathcal{C}_{4}} \gamma_{s(h) \rightarrow t(j)}+\sum_{(h, j) \in \mathcal{C}_{4}} \gamma_{t(j) \rightarrow \sqcup} & \geq \sum_{(h, j) \in \mathcal{C}_{4}} \gamma_{s(h) \rightarrow \sqcup} . \\
\sum_{(j, k) \in \mathcal{C}_{5}} \gamma_{\sqcup \rightarrow t(j)}+\sum_{(j, k) \in \mathcal{C}_{5}} \gamma_{t(j) \rightarrow u(k)} & \geq \sum_{(j, k) \in \mathcal{C}_{5}} \gamma_{\sqcup \rightarrow u(k)} . \\
\sum_{(h, j, k) \in \mathcal{C}_{6}} \gamma_{s(h) \rightarrow t(j)}+\sum_{(h, j, k) \in \mathcal{C}_{6}} \gamma_{t(j) \rightarrow u(k)} & \geq \sum_{(h, j, k) \in \mathcal{C}_{6}} \gamma_{s(h) \rightarrow u(k)} .
\end{aligned}
$$

Por (iii) e pela definição de $\mathcal{C}_{7}$ segue que

$$
\sum_{(h, j, k) \in \mathcal{C}_{7}} \gamma_{s(h) \rightarrow t(j)}+\sum_{(h, j, k) \in \mathcal{C}_{7}} \gamma_{t(j) \rightarrow u(k)} \geq \sum_{(h, j, k) \in \mathcal{C}_{7}}\left(\gamma_{s(h) \rightarrow \sqcup}+\gamma_{\sqcup \rightarrow u(k)}\right) .
$$

Por (4.9), (4.10), (4.11), (4.12) e (4.13), temos que

$$
v \mathrm{~A}_{\gamma}(A)+v \mathrm{~A}_{\gamma}(B) \geq v \mathrm{~A}_{\gamma}(C)+\left|\mathcal{C}_{3}\right| \lambda
$$

Tomando $k=\left|\mathcal{C}_{3}\right|$, para finalizar a prova, basta mostrar que $|A| \leq|C|+\left|\mathcal{C}_{3}\right|$ e $|B| \leq|C|+\left|\mathcal{C}_{3}\right|$.

Desde que

$$
\begin{aligned}
|A|=\sum_{i}\left|\mathcal{C}_{i}\right|-\left|\mathcal{C}_{2}\right| & \leq \sum_{i}\left|\mathcal{C}_{i}\right|, \\
|B|=\sum_{i}\left|\mathcal{C}_{i}\right|-\left|\mathcal{C}_{1}\right| & \leq \sum_{i}\left|\mathcal{C}_{i}\right|, \\
|C|=\sum_{i}\left|\mathcal{C}_{i}\right|-\left|\mathcal{C}_{3}\right|+\left|\mathcal{C}_{7}\right| & \geq \sum_{i}\left|\mathcal{C}_{i}\right|-\left|\mathcal{C}_{3}\right|,
\end{aligned}
$$

temos $|A| \leq \sum_{i}\left|\mathcal{C}_{i}\right| \leq|C|+\left|\mathcal{C}_{3}\right|$ e $|B| \leq \sum_{i}\left|\mathcal{C}_{i}\right| \leq|C|+\left|\mathcal{C}_{3}\right|$.

Lema 46. Seja $\gamma$ uma matriz de pontuação. Então, optA $\mathrm{A}_{\gamma}(s, u) \leq \operatorname{opt} \mathrm{A}_{\gamma}(s, t)+\operatorname{opt} \mathrm{A}_{\gamma}(t, u)$ para cada $s, t, u \in \Sigma^{*}$ se e somente se, para cada a, b, c $\in \Sigma$,

(i) $\gamma_{\mathrm{a} \rightarrow \sqcup} \leq \gamma_{\mathrm{a} \rightarrow \mathrm{b}}+\gamma_{\mathrm{b} \rightarrow \sqcup}$,

(ii) $\gamma_{\mathrm{S} \rightarrow \mathrm{a}} \leq \gamma_{\boldsymbol{\sqcup} \rightarrow \mathrm{b}}+\gamma_{\mathrm{b} \rightarrow \mathrm{a}}$,

(iii) $\min \left\{\gamma_{\mathrm{a} \rightarrow \mathrm{c}}, \gamma_{\mathrm{a} \rightarrow \sqcup}+\gamma_{\mathrm{u} \rightarrow \mathrm{c}}\right\} \leq \gamma_{\mathrm{a} \rightarrow \mathrm{b}}+\gamma_{\mathrm{b} \rightarrow \mathrm{c}}$;

(iv) $\gamma_{\mathrm{b} \rightarrow \sqcup}+\gamma_{\iota^{\rightarrow b}} \geq 0$.

Prova. Sejam a, b, c $\in \Sigma$ e suponha que $\operatorname{opt}_{\gamma}(s, u) \leq \operatorname{opt} \mathrm{A}_{\gamma}(s, t)+\operatorname{opt} \mathrm{A}_{\gamma}(t, u)$ para cada $s, t, u \in \Sigma^{*}$. Seguem de (4.2), (4.3) e (4.4) que

$$
\begin{aligned}
& \gamma_{\mathrm{a} \rightarrow \sqcup}=\operatorname{opt}_{\gamma}(\mathrm{a}, \epsilon) \leq \operatorname{opt} \mathrm{A}_{\gamma}(\mathrm{a}, \mathrm{b})+\operatorname{opt} \mathrm{A}_{\gamma}\left(\mathrm{b},{ }_{\sqcup}\right) \\
& \leq \gamma_{\mathrm{a} \rightarrow \mathrm{b}}+\gamma_{\mathrm{b} \rightarrow \boldsymbol{b}} \text {, } \\
& \gamma_{\hookrightarrow \rightarrow \mathrm{a}}=\operatorname{optA}_{\gamma}(\epsilon, \mathrm{a}) \leq \operatorname{opt} \mathrm{A}_{\gamma}(\sqcup, \mathrm{b})+o p t \mathrm{~A}_{\gamma}(\mathrm{b}, \mathrm{a}) \\
& \leq \gamma_{\mathrm{u} \rightarrow \mathrm{b}}+\gamma_{\mathrm{b} \rightarrow \mathrm{a}} \text {, }
\end{aligned}
$$


$\mathrm{e}$

$$
\begin{aligned}
\min \left\{\gamma_{\mathrm{a} \rightarrow \mathrm{c}}, \gamma_{\mathrm{a} \rightarrow_{\sqcup}}+\gamma_{\mathrm{b} \rightarrow \mathrm{c}}\right\} & =\operatorname{optA}_{\gamma}(\mathrm{a}, \mathrm{c}) \\
& \leq \operatorname{optA}_{\gamma}(\mathrm{a}, \mathrm{b})+\operatorname{opt} \mathrm{A}_{\gamma}(\mathrm{b}, \mathrm{c}) \leq \gamma_{\mathrm{a} \rightarrow \mathrm{b}}+\gamma_{\mathrm{b} \rightarrow \mathrm{c}}
\end{aligned}
$$

Portanto, se opt $\mathrm{A}_{\gamma}(s, u) \leq o p t \mathrm{~A}_{\gamma}(s, t)+o p t \mathrm{~A}_{\gamma}(t, u)$ para cada $s, t, u \in \Sigma^{*}$ valem $(i),(i i)$ e $(i i i)$ para cada a, b, $\mathrm{c} \in \Sigma$. Para mostrar que também vale $(i v)$, assumimos que $\gamma_{\mathrm{b} \rightarrow \sqcup}+\gamma_{\llcorner\rightarrow \mathrm{b}} \neq 0$ pois se $\gamma_{\mathrm{b} \rightarrow}+\gamma_{\llcorner\rightarrow \mathrm{b}}=0$, então vale $(i v)$ trivialmente.

Seja

$$
n>\frac{\operatorname{optA}_{\gamma}(\mathrm{a}, \mathrm{c})-\left(\gamma_{\mathrm{a} \rightarrow \sqcup}+\gamma_{\lrcorner \rightarrow \mathrm{c}}\right)}{\gamma_{\mathrm{b} \rightarrow \sqcup}+\gamma_{\sqcup \rightarrow \mathrm{b}}}
$$

um inteiro positivo. Desde que $\left[\mathrm{a}_{\sqcup}{ }^{n}, \sqcup^{\left.\mathrm{b}^{n}\right]}\right.$ e $\left[\mathrm{b}^{n}{ }_{\sqcup},{ }^{n} \mathrm{c}\right]$ são alinhamentos de $\left(\mathrm{a}, \mathrm{b}^{n}\right)$ e $\left(\mathrm{b}^{n}, \mathrm{c}\right)$ respectivamente, segue que

$$
\begin{aligned}
\operatorname{opt}_{\gamma}(\mathrm{a}, \mathrm{c}) & \leq \operatorname{optA}_{\gamma}\left(\mathrm{a}, \mathrm{b}^{n}\right)+\operatorname{opt} \mathrm{A}_{\gamma}\left(\mathrm{b}^{n}, \mathrm{c}\right) \\
& \leq v \mathrm{~A}_{\gamma}\left(\left[\mathrm{a}_{\sqcup}{ }^{n}, \mathrm{~b}^{n}\right]\right)+v \mathrm{~A}_{\gamma}\left(\left[\mathrm{b}^{n} \sqcup,{ }_{\sqcup}{ }^{n} \mathrm{c}\right]\right) \\
& =\gamma_{\mathrm{a} \rightarrow \sqcup}+n \gamma_{\sqcup \rightarrow \mathrm{b}}+n \gamma_{\mathrm{b} \rightarrow \sqcup}+\gamma_{\sqcup \rightarrow \mathrm{c}},
\end{aligned}
$$

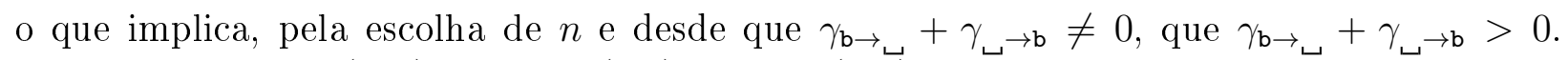
Portanto, se optA $\mathrm{A}_{\gamma}(s, u) \leq o p t \mathrm{~A}_{\gamma}(s, t)+o p t \mathrm{~A}_{\gamma}(t, u)$ para cada $s, t, u \in \Sigma^{*}$, então valem (i), (ii), (iii) e (iv) para a, b, c $\in \Sigma$.

Reciprocamente, suponha que valem $(i),(i i),(i i i)$ e $(i v)$ para a, b, c $\in \Sigma$. Sejam $s, t, u \in$ $\Sigma^{*}$ e $A, B$ alinhamentos A-ótimos de $(s, t)$ e de $(t, u)$ respectivamente. Segue, da Proposição 45 , que existem um inteiro $k \geq 0$ e um alinhamento $C$ de $(s, u)$ tais que,

$$
v \mathrm{~A}_{\gamma}(C)+0 k \leq v \mathrm{~A}_{\gamma}(A)+v \mathrm{~A}_{\gamma}(B) .
$$

Portanto, desde que $A$ e $B$ são alinhamentos A-ótimos de $(s, t)$ e $(t, u)$, e $C$ é um alinhamento de $(s, u)$, segue que

$$
\operatorname{opt}_{\gamma}(s, u) \leq v \mathrm{~A}_{\gamma}(C)=v \mathrm{~A}_{\gamma}(C)+0 k \leq v \mathrm{~A}_{\gamma}(A)+v \mathrm{~A}_{\gamma}(B)=o p t \mathrm{~A}_{\gamma}(s, t)+o p t \mathrm{~A}_{\gamma}(t, u) .
$$

Portanto, se valem $(i),(i i),(i i i)$ e (iv) para cada a, b, c $\in \Sigma$, então opt $\mathrm{A}_{\gamma}(s, u) \leq \operatorname{opt} \mathrm{A}_{\gamma}(s, t)+$ $\operatorname{opt}_{\gamma}(t, u)$ para cada $s, t, u \in \Sigma^{*}$.

A Tabela 4.2 resume os resultados que caracterizam as matrizes de pontuação que induzem as propriedades de funções métricas na função opt $\mathrm{A}_{\gamma}$. Ela é construída usando a Tabela 4.1 e os resultados obtidos dos Lemas 41, 42, 43, 44 e 46. Para cada classe de funções, é assinalado um conjunto de propriedades suficientes e necessárias de suas matrizes de pontuação.

O seguinte teorema prova um dos resultados discutidos no início deste capítulo.

Teorema 47. Seja $\gamma$ uma matriz de pontuação. Então, opt $\mathrm{A}_{\gamma}$ é uma métrica em $\Sigma^{*}$ se e somente se $\gamma \in \mathbb{M}^{\mathrm{A}}$.

Prova. Suponha que opt $\mathrm{A}_{\gamma}$ é uma métrica. Para provar que nesse caso $\gamma \in \mathbb{M}^{\mathrm{A}}$, basta mostrar que, para cada $a, b, c \in \Sigma$, valem

(i) $\gamma_{\mathrm{a} \rightarrow_{\llcorner}}=\gamma_{\lrcorner \rightarrow \mathrm{a}}>0$;

(ii) $\gamma_{\mathrm{a} \rightarrow \mathrm{b}}>0$ se $\mathrm{a} \neq \mathrm{b}$ e $\gamma_{\mathrm{a} \rightarrow \mathrm{b}}=0$ se $\mathrm{a}=\mathrm{b}$; 


\begin{tabular}{|c|c|c|c|c|c|c|c|}
\hline & & $\mathcal{P r}$ & $\mathcal{S}$ & $\mathcal{H}$ & $P$ & $Q$ & $\mathcal{M}$ \\
\hline (a) & $D(\gamma)$ não possui ciclo negativo & $\mathrm{x}$ & $\mathrm{x}$ & $\mathrm{x}$ & $\mathrm{x}$ & $\mathrm{x}$ & $\mathrm{x}$ \\
\hline (b) & $\gamma_{\mathrm{a} \rightarrow \mathrm{a}}=0$ ou $\gamma_{\mathrm{a} \rightarrow \sqcup}+\gamma_{\sqcup \rightarrow \mathrm{a}}=0$ & $\mathrm{x}$ & $\mathrm{x}$ & $\mathrm{x}$ & $\mathrm{x}$ & $\mathrm{x}$ & $\mathrm{x}$ \\
\hline (c) & $\gamma_{\mathrm{a} \rightarrow \mathrm{b}}, \gamma_{\mathrm{L} \rightarrow \mathrm{b}}, \gamma_{\mathrm{a} \rightarrow \mathrm{b}} \geq 0$ & $\mathrm{x}$ & $\mathrm{x}$ & $\mathrm{x}$ & $\mathrm{x}$ & $\mathrm{x}$ & $\mathrm{x}$ \\
\hline (d) & $\gamma_{\mathrm{a} \rightarrow \mathrm{a}} \geq 0$ & & $\mathrm{x}$ & & & $\mathrm{x}$ & $\mathrm{x}$ \\
\hline (e) & $\gamma_{a \rightarrow b}, \gamma_{\lrcorner \rightarrow a}>0$ e $\gamma_{a \rightarrow b}>0$ se $a \neq b$ & & $\mathrm{x}$ & & & $\mathrm{x}$ & $\mathrm{x}$ \\
\hline (f) & $\gamma_{\mathrm{a} \rightarrow \sqcup}=\gamma_{\sqcup \rightarrow \mathrm{a}}$ & & $\mathrm{x}$ & & $\mathrm{x}$ & & $\mathrm{x}$ \\
\hline (g) & $\begin{array}{l}\text { se } \gamma_{a \rightarrow b}<\gamma_{a \rightarrow}+\gamma_{\llcorner} \rightarrow b \\
\gamma_{a \rightarrow b}=\gamma_{b \rightarrow a}\end{array}$ & & $x$ & & $\mathrm{x}$ & & $\mathrm{x}$ \\
\hline (h) & $\gamma_{\mathrm{a} \rightarrow_{\sqcup}} \leq \gamma_{\mathrm{a} \rightarrow \mathrm{b}}+\gamma_{\mathrm{b} \rightarrow_{\sqcup}}$ & & & $\mathrm{x}$ & $\mathrm{x}$ & $\mathrm{x}$ & $\mathrm{x}$ \\
\hline (i) & 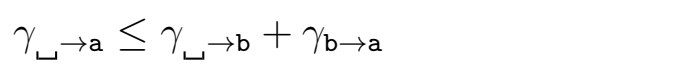 & & & $\mathrm{x}$ & $\mathrm{x}$ & $\mathrm{x}$ & $\mathrm{x}$ \\
\hline (j) & 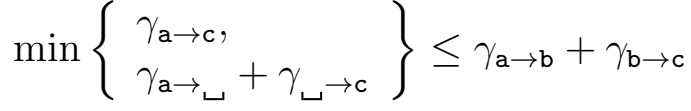 & & & $\mathrm{x}$ & $\mathrm{x}$ & $\mathrm{x}$ & $\mathrm{x}$ \\
\hline$(\mathrm{k})$ & $\gamma_{\mathrm{b} \rightarrow}+\gamma_{\mathrm{b} \rightarrow \mathrm{b}} \geq 0$ & & & $\mathrm{x}$ & $\mathrm{x}$ & $\mathrm{x}$ & $\mathrm{X}$ \\
\hline
\end{tabular}

Tabela 4.2: Considerando o critério opt $\mathrm{A}_{\gamma}$, para cada classe de funçôes pramétrica(P्Pr), semimétrica $(S)$, hemimétrica $(\mathcal{H})$, pseudométrica $(\mathcal{P})$, quasimétrica $(Q)$ e métrica $(\mathcal{M})$, um $\mathrm{x}$ indica que a condição assinalada correspondente para cada $\mathrm{a}, \mathrm{b}, \mathrm{c} \in \Sigma$ é necessária para que $\gamma$ induza as propriedades que definem as funçôes dessa classe. Este é um quadro resumo que relaciona as definiçôes de classes de função opt $\mathrm{A}_{\gamma}$ com os resultados obtidos pelos Lemas 41, 42, 43, 44 e 46 para cada a, b, $\mathrm{c} \in \Sigma$. 
(iii) se $\gamma_{\mathrm{a} \rightarrow \mathrm{b}}<\gamma_{\mathrm{a} \rightarrow \sqcup}+\gamma_{\llcorner\rightarrow \mathrm{b}}$, estão $\gamma_{\mathrm{a} \rightarrow \mathrm{b}}=\gamma_{\mathrm{b} \rightarrow \mathrm{a}}$.

(iv) $\gamma_{\mathrm{a} \rightarrow_{\sqcup}} \leq \gamma_{\mathrm{a} \rightarrow \mathrm{b}}+\gamma_{\mathrm{b} \rightarrow_{\sqcup}}$;

(v) $\min \left\{\gamma_{\mathrm{a} \rightarrow \mathrm{c}}, \gamma_{\mathrm{a} \rightarrow \sqcup}+\gamma_{{ }^{-} \rightarrow \mathrm{c}}\right\} \leq \gamma_{\mathrm{a} \rightarrow \mathrm{b}}+\gamma_{\mathrm{b} \rightarrow \mathrm{c}}$.

Usamos a Tabela 4.2 para verificar cada uma das condições necessárias para opt $\mathrm{A}_{\gamma}$ ser

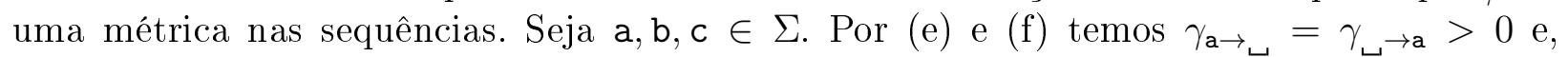
portanto, vale $(i)$. Como vale $(i)$, temos que $\gamma_{a \rightarrow}+\gamma_{\mathrm{s} \rightarrow \mathrm{a}} \neq 0$, o que implica, por (b), que $\gamma_{\mathrm{a} \rightarrow \mathrm{a}}=0$ e como vale (e), temos que $\gamma_{\mathrm{a} \rightarrow \mathrm{b}}>0$ se $\mathrm{a} \neq \mathrm{b}$. Segue que vale $(i i)$. Como valem (g), (h) e (j), temos que valem $(i i i),(i v)$ e $(v)$. Portanto, se opt $\mathrm{A}_{\gamma}$ é uma métrica, então valem $(i),(i i),(i i i),(i v)$ e $(v)$ para cada $\mathrm{a}, \mathrm{b}, \mathrm{c} \in \Sigma$, o que implica que $\gamma \in \mathbb{M}^{\mathrm{A}}$.

Reciprocamente, suponha que $\gamma \in \mathbb{M}^{\mathrm{A}}$, ou seja, valem as condições $(i)$ a $(v)$. Para mostrar que essas condições são suficientes para opt $\mathrm{A}_{\gamma}$ ser uma métrica, basta verificar que as condições necessárias (a) a (k) assinaladas na Tabela 4.2 são satisfeitas. Usando (i) e (ii), é imediata a verificação das condições (a) a (f) e da condição (k). Usando (iii), (iv) e (v), verificamos que as condições $(\mathrm{g}),(\mathrm{h})$ e (j) são satisfeitas. Resta mostrar que vale (i).

Se $\gamma_{\mathrm{b} \rightarrow \mathrm{a}} \geq \gamma_{\mathrm{b} \rightarrow \sqcup}+\gamma_{\sqcup \rightarrow \mathrm{a}}$, então, desde que vale $(\mathrm{k})$, segue que

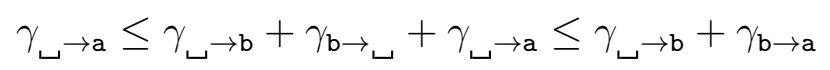

e a prova termina. Se $\gamma_{\mathrm{b} \rightarrow \mathrm{a}}<\gamma_{\mathrm{b} \rightarrow \sqcup}+\gamma_{\iota_{\rightarrow \mathrm{a}}}$, então segue de (iii) que $\gamma_{\mathrm{b} \rightarrow \mathrm{a}}=\gamma_{\mathrm{a} \rightarrow \mathrm{b}}$, o que implica, usando $(i)$ e $(i v)$, que

$$
\gamma_{\sqcup \rightarrow \mathrm{a}}=\gamma_{\mathrm{a} \rightarrow \sqcup} \leq \gamma_{\mathrm{a} \rightarrow \mathrm{b}}+\gamma_{\mathrm{b} \rightarrow \sqcup}=\gamma_{\lrcorner \rightarrow \mathrm{b}}+\gamma_{\mathrm{b} \rightarrow \mathrm{a}}
$$

Portanto, se $\gamma \in \mathbb{M}^{\mathrm{A}}$, então opt $\mathrm{A}_{\gamma}$ é uma métrica.

\subsection{Distância normalizada de alinhamento}

Nesta seção mostramos as condições necessárias e suficientes para a matriz de pontuação $\gamma$ induzir propriedades de uma métrica na função opt $\mathrm{N}_{\gamma}$.

Lema 48. Seja $\gamma$ uma matriz de pontuação. Então, opt $\mathrm{A}_{\gamma} \in \operatorname{Pr}$ se e somente se $\operatorname{opt} \mathrm{N}_{\gamma} \in \operatorname{Pr}$.

Prova. Sejam $s, t \in \Sigma^{*}$ sequências arbitrárias. Sendo $s, t$ sequências arbitrárias, para provar

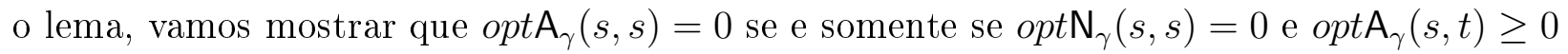
se e somente se $\operatorname{opt} \mathrm{N}_{\gamma}(s, t) \geq 0$.

Inicialmente, mostramos que se opt $\mathrm{A}_{\gamma}(s, s)=0$, então $\operatorname{opt} \mathrm{N}_{\gamma}(s, s)=0$. Desde que optA $\mathrm{A}_{\gamma}(\epsilon, \epsilon)=$ opt $\mathrm{N}_{\gamma}(\epsilon, \epsilon)=0$, para o caso $s=\epsilon$, o argumento está provado. Assumimos então que $s \neq \epsilon$. Suponha que opt $\mathrm{A}_{\gamma}(s, s)=0$. Seja $A$ um alinhamento A-ótimo de $(s, s)$. Desde que optA $(s, s)=0$, segue que $v \mathrm{~A}_{\gamma}(A)=0$ e, desde que $s \neq \epsilon$, temos que $|A|>0$. Segue que

$$
\operatorname{opt}_{\gamma}(s, s) \leq v \mathrm{~N}_{\gamma}(A)=\frac{v \mathrm{~A}_{\gamma}(A)}{|A|}=0 .
$$

Seja $B$ um alinhamento N-ótimo de $(s, s)$. Desde que $s \neq \epsilon$, segue que $|B|>0$, o que implica, desde que $\operatorname{opt}_{\gamma}(s, s)=0$ e $v \mathrm{~A}_{\gamma}(B) \geq \operatorname{opt} \mathrm{A}_{\gamma}(s, s)$, que

$$
o p t \mathrm{~N}_{\gamma}(s, s)=\frac{v \mathrm{~A}_{\gamma}(B)}{|B|} \geq \frac{o p t \mathrm{~A}_{\gamma}(s, s)}{|B|}=0 .
$$


Segue de (4.14) que se opt $\mathrm{A}_{\gamma}(s, s)=0$, então opt $\mathrm{N}_{\gamma}(s, s)=0$.

Argumentos similares podem ser usados para provar que opt $\mathrm{N}_{\gamma}(s, s)=0$ implica que $o p t \mathrm{~A}_{\gamma}(s, s)=0$ e que opt $\mathrm{A}_{\gamma}(s, t) \geq 0$ se e somente se opt $\mathrm{N}_{\gamma}(s, t) \geq 0$.

Corolário 49. Sejam $\gamma$ uma matriz de pontuação. Então, opt $\mathrm{N}_{\gamma} \in \operatorname{Pr}$ se e somente se valem

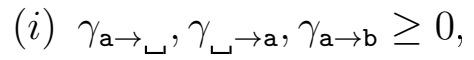

(ii) $\gamma_{\mathrm{a} \rightarrow \mathrm{a}}=0$ ou $\gamma_{\mathrm{a} \rightarrow \boldsymbol{b}}+\gamma_{\mathrm{s} \rightarrow \mathrm{a}}=0$,

para cada $a, b \in \Sigma$.

Prova. Suponha que opt $\mathrm{A}_{\gamma} \in \mathcal{P}$ r. Logo, pela Tabela 4.2, Propriedades (b) e (c), valem as condições $(i)$ e $(i i)$ para cada $\mathrm{a}, \mathrm{b} \in \Sigma$.

Reciprocamente, suponha que valem as condições $(i)$ e $(i i)$ para cada $\mathrm{a}, \mathrm{b} \in \Sigma$. Para provar que opt $\mathrm{A}_{\gamma} \in \mathcal{P r}$, basta verificar que valem as Propriedades (a), (b) e (c) da Tabela 4.2. É imediato que valem (b) e (c), e desde que não há entrada negativa em $\gamma$, segue que $D(\gamma)$ não pode ter ciclo negativo, o que implica que vale (a).

Pelos argumentos acima, temos que opt $\mathrm{A}_{\gamma} \in \mathcal{P r}$ se e somente se valem $(i)$ e $(i i)$ para cada a, $\mathrm{b} \in \Sigma$. Segue do Lema 48 que opt $\mathrm{N}_{\gamma} \in \mathcal{P r}$ se e somente se valem $(i)$ e $(i i)$.

Lema 50. Seja opt $\mathrm{N}_{\gamma} \in \mathcal{P r}$. Então, opt $\mathrm{N}_{\gamma}(s, t)>0$ para cada $s \neq t \in \Sigma^{*}$, se e somente se $\gamma_{\mathrm{a} \rightarrow}, \gamma_{\boldsymbol{}} \rightarrow \mathrm{a}, \gamma_{\mathrm{a} \rightarrow \mathrm{b}}>0$ para cada $\mathrm{a} \neq \mathrm{b} \in \Sigma$.

Prova. Seja $\mathrm{a} \neq \mathrm{b} \in \Sigma$ e suponha que $\operatorname{opt} \mathrm{N}_{\gamma}(s, t)>0$ para cada $s \neq t \in \Sigma^{*}$. Então,

$$
\begin{aligned}
\gamma_{\mathrm{a} \rightarrow \boldsymbol{}} & =v \mathrm{~N}_{\gamma}\left(\left[\mathrm{a},,_{\sqcup}\right]\right)=\operatorname{opt} \mathrm{N}_{\gamma}(\mathrm{a}, \epsilon)>0 \\
\gamma_{\sqcup \rightarrow \mathrm{a}} & =v \mathrm{~N}_{\gamma}([\mathrm{c}, \mathrm{a}])=\operatorname{opt} \mathrm{N}_{\gamma}(\epsilon, \mathrm{a})>0 \\
\gamma_{\mathrm{a} \rightarrow \mathrm{b}} & =v \mathrm{~N}_{\gamma}([\mathrm{a}, \mathrm{b}]) \geq \operatorname{opt} \mathrm{N}_{\gamma}(\mathrm{a}, \mathrm{b})>0
\end{aligned}
$$

Portanto, se $\operatorname{opt} \mathrm{N}_{\gamma}(s, t)>0$ para cada $s \neq t \in \Sigma^{*}$, então $\gamma_{\mathrm{a} \rightarrow\lrcorner}, \gamma_{\boldsymbol{L}_{\rightarrow}}, \gamma_{\mathrm{a} \rightarrow \mathrm{b}}>0$ para cada $\mathrm{a} \neq \mathrm{b} \in \Sigma$.

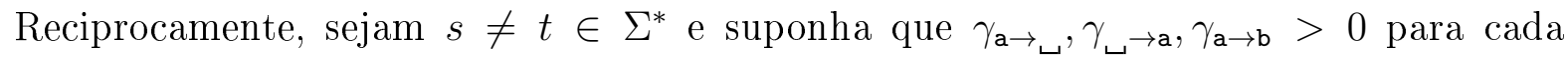
$\mathrm{a} \neq \mathrm{b} \in \Sigma$. Como opt $\mathrm{N}_{\gamma} \in \mathcal{P r}$, temos que $\gamma_{\mathrm{a} \rightarrow \mathrm{a}} \geq 0$ pelo Corolário 49. Segue do Lema 43 que $\operatorname{optA}_{\gamma}(s, t)>0$. Seja $A$ um alinhamento N-ótimo de $(s, t)$. Segue que

$$
o p t \mathrm{~N}_{\gamma}(s, t)=v \mathrm{~N}_{\gamma}(A)=\frac{v \mathrm{~A}_{\gamma}(A)}{|A|} \geq \frac{o p t \mathrm{~A}_{\gamma}(s, t)}{|A|}>0 .
$$

Portanto, se $\gamma_{\mathrm{a} \rightarrow}, \gamma_{\boldsymbol{b} \rightarrow \mathrm{a}}, \gamma_{\mathrm{a} \rightarrow \mathrm{b}}>0$ para cada $\mathrm{a} \neq \mathrm{b} \in \Sigma$, então opt $\mathrm{N}_{\gamma}(s, t)>0$ para cada $s \neq t \in \Sigma^{*}$.

Denotamos por $Q_{\max }$ o valor de $\max _{\mathrm{a} \in \Sigma}\left\{\gamma_{\mathrm{a} \rightarrow \boldsymbol{\iota}}, \gamma_{\boldsymbol{\iota} \rightarrow \mathrm{a}}\right\}$ e $q=\arg \max _{\mathrm{a} \in \Sigma}\left\{\gamma_{\mathrm{a} \rightarrow \boldsymbol{\iota}}, \gamma_{\boldsymbol{\iota}} \rightarrow \mathrm{a}\right\}$.

Proposição 51. Sejam $s, t \in \Sigma^{*}$. Se $s \neq \epsilon$ ou $t \neq \epsilon$, então $\operatorname{opt} \mathrm{N}_{\gamma}(s, t) \leq Q_{\max }$.

Prova. Suponha que $s \neq \epsilon$ ou $t \neq \epsilon$. Logo, qualquer alinhamento $A$ de $(s, t)$ é tal que $|A|>0$, o que implica que $v \mathrm{~N}_{\gamma}(A)=v \mathrm{~A}_{\gamma}(A) /|A|$. Portanto,

$$
\operatorname{opt}_{\gamma}(s, t) \leq v \mathrm{~N}_{\gamma}\left(\left[\begin{array}{cc}
s & \iota^{|t|} \\
\sqcup^{|s|} & t
\end{array}\right]\right) \leq \frac{|s| Q_{\max }+|t| Q_{\max }}{|s|+|t|}=Q_{\max } .
$$

Logo, se $s \neq \epsilon$ ou $t \neq \epsilon$, então $\operatorname{opt} \mathrm{N}_{\gamma}(s, t) \leq Q_{\text {max }}$. 
Proposição 52. Sejam opt $\mathrm{N}_{\gamma} \in \mathcal{P r}$, a $\in \Sigma$ e $s, t \in \Sigma^{*}$.

1. Se $Q_{\max }=0$, então opt $\mathrm{N}_{\gamma}(s, t)=\gamma_{\mathrm{a} \rightarrow\lrcorner}=\gamma_{\lrcorner \rightarrow \mathrm{a}}=0$,

2. Se $Q_{\max } \neq 0$, então $\gamma_{q \rightarrow \sqcup}+\gamma_{\lrcorner \rightarrow q}>0$ e $\gamma_{q \rightarrow q}=0$.

Prova. Suponha $Q_{\max }=0$. Como opt $\mathrm{N}_{\gamma} \in \mathcal{P} r$, temos que opt $\mathrm{N}_{\gamma}(s, t) \geq 0$. Se $s=\epsilon$ e $t=\epsilon$, então $\operatorname{opt} \mathrm{N}_{\gamma}(s, t)=0$, e se $s \neq \epsilon$ ou $t \neq \epsilon$, então, pela Proposição 51 , temos que $\operatorname{opt} \mathrm{N}_{\gamma}(s, t) \leq Q_{\max }=0$. Logo, opt $\mathrm{N}_{\gamma}(s, t)=0$. Desde que esse resultado vale para quaisquer $s, t$, segue que opt $\mathrm{N}_{\gamma}(\mathrm{a}, \epsilon)=0$ e $\operatorname{opt} \mathrm{N}_{\gamma}(\epsilon, \mathrm{a})=0$. Desde que [a, _] e [๖, a ] são os únicos alinhamentos de $(\mathrm{a}, \epsilon)$ e de $(\epsilon, \mathrm{a})$ respectivamente, segue que

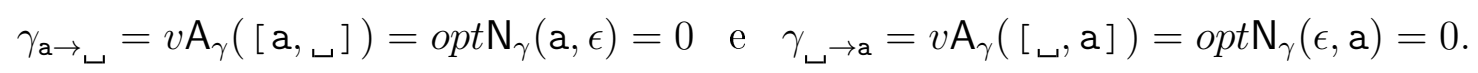

Portanto, se $Q_{\max }=0$, então $\operatorname{opt} \mathrm{N}_{\gamma}(s, t)=\gamma_{\mathrm{a} \rightarrow\lrcorner}=\gamma_{\mathrm{s}_{\rightarrow \mathrm{a}}}=0$.

Suponha $Q_{\max } \neq 0$. Como opt $\mathrm{N}_{\gamma} \in \mathcal{P r}$, pelo Corolário 49 temos que $\gamma_{q \rightarrow \sqcup}, \gamma_{\sqcup \rightarrow q} \geq 0$.

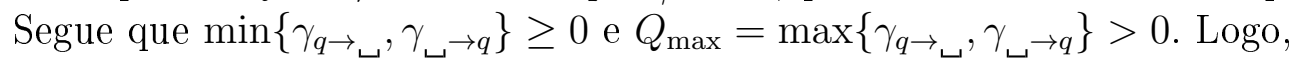

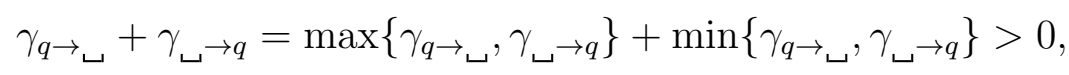

o que implica pelo Corolário 49 que $\gamma_{q \rightarrow q}=0$. Portanto, se $Q_{\max } \neq 0$, então $\gamma_{q \rightarrow}+\gamma_{\sqcup \rightarrow q}>0$ e $\gamma_{q \rightarrow q}=0$.

Fato 53. Sejam $x, z, k, w$ números reais. Se $k \geq 0$ e $w>0$, então

$$
\frac{k x+z}{k+w} \geq \min \left\{x, \frac{z}{w}\right\}
$$

Fato 54. Sejam os números $n>0, m>0, x \geq 0$. Se $n>x / m$, então

$$
m>\frac{x}{n} \geq \frac{x}{n+1} \geq \frac{x}{n+2} .
$$

Proposição 55. Considere que $\operatorname{opt} \mathrm{N}_{\gamma} \in \operatorname{Pr}$ e $Q_{\max } \neq 0$. Seja a, b $\in \Sigma$. Se

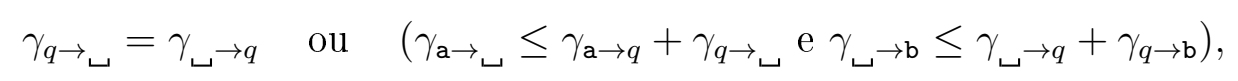

então existe $n_{0}$ tal que para cada inteiro $n>n_{0}$ temos que

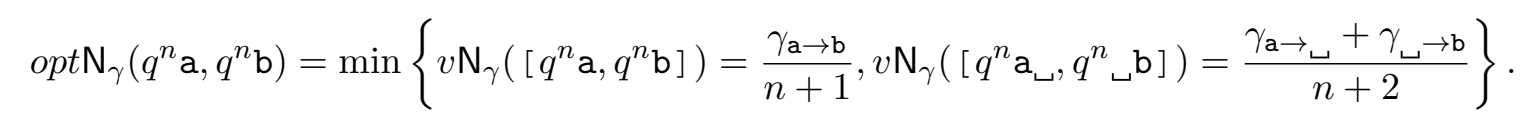

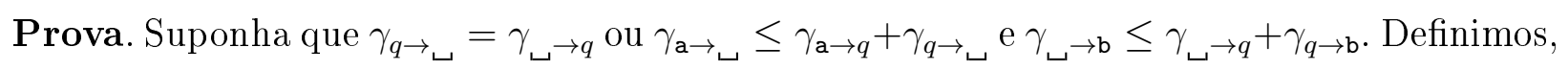

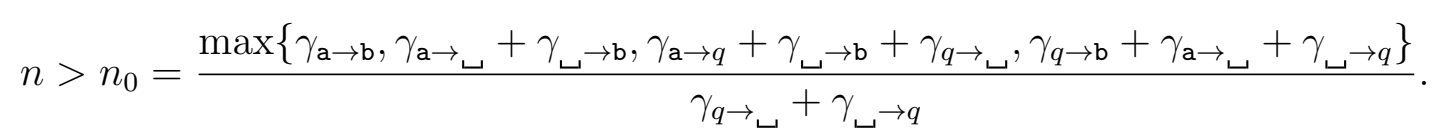

Sendo opt $\mathrm{N}_{\gamma} \in \mathcal{P r}$, segue do Corolário 49 que

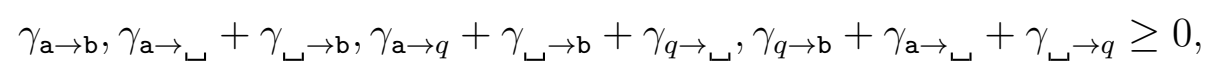

e sendo $Q_{\max } \neq 0$, segue da Proposição 52 que $\gamma_{q \rightarrow \sqcup}+\gamma_{\sqcup \rightarrow q}>0$ e $\gamma_{q \rightarrow q}=0$. 
Como $\gamma_{q \rightarrow q}=0$, temos que

$$
v \mathrm{~N}_{\gamma}\left(\left[\begin{array}{ll}
q^{n} & \mathrm{a} \\
q^{n} & \mathrm{~b}
\end{array}\right]\right)=\frac{\gamma_{\mathrm{a} \rightarrow \mathrm{b}}}{n+1} \quad \text { e } \quad v \mathrm{~N}_{\gamma}\left(\left[\begin{array}{ccc}
q^{n} & \mathrm{a} & \sqcup \\
q^{n} & \iota & \mathrm{b}
\end{array}\right]\right)=\frac{\gamma_{\mathrm{a} \rightarrow}+\gamma_{\llcorner} \rightarrow \mathrm{b}}{n+2}
$$

Logo, para provar a proposição, mostramos que

$$
v \mathrm{~N}_{\gamma}\left(\left[s^{\prime}, t^{\prime}\right]\right) \geq \min \left\{\frac{\gamma_{\mathrm{a} \rightarrow \mathrm{b}}}{n+1}, \frac{\gamma_{\mathrm{a} \rightarrow}+\gamma_{\sqcup \rightarrow \mathrm{b}}}{n+2}\right\}
$$

para cada alinhamento $\left[s^{\prime}, t^{\prime}\right]$ de $\left(q^{n} \mathrm{a}, q^{n} \mathrm{~b}\right)$.

Seja $k$ a quantidade de símbolos $\_$em $s^{\prime}$. Segue que a quantidade de símbolos ${ }_{\longleftarrow} \mathrm{em}$ $t^{\prime}$ é também $k$ e $\left|\left[s^{\prime}, t^{\prime}\right]\right|=k+n+1$. Examinamos quatro casos, abrangendo todos os alinhamentos possíveis de $\left(q^{n} \mathrm{a}, q^{n} \mathrm{~b}\right)$.

C-1 [a, b] alinha em $\left[s^{\prime}, t^{\prime}\right]$.

Neste caso, $k \geq 0$. Sendo $\gamma_{q \rightarrow q}=0$, temos

$$
\begin{aligned}
v \mathrm{~N}_{\gamma}\left(\left[s^{\prime}, t^{\prime}\right]\right) & =\frac{k\left(\gamma_{q \rightarrow}+\gamma_{\sqcup \rightarrow q}\right)+\gamma_{\mathrm{a} \rightarrow \mathrm{b}}}{k+n+1} \\
& \geq \min \left\{\gamma_{q \rightarrow \sqcup}+\gamma_{\sqcup \rightarrow q}, \frac{\gamma_{\mathrm{a} \rightarrow \mathrm{b}}}{n+1}\right\} \\
& =\frac{\gamma_{\mathrm{a} \rightarrow \mathrm{b}}}{n+1} .
\end{aligned}
$$

A Desigualdade (4.15), desde que $k \geq 0$ e $n+1>0$, segue do Fato 53. A Igual-

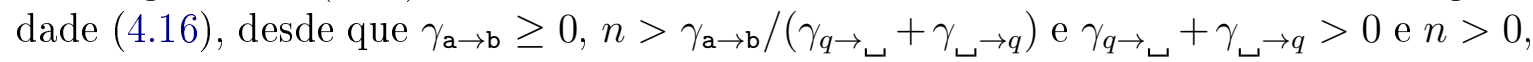
segue do Fato 54.

C-2 $\left[\mathrm{a},{ }_{\sqcup}\right]$ e $[\sqcup, \mathrm{b}]$ alinham em $\left[s^{\prime}, t^{\prime}\right]$.

Neste caso, $k \geq 1$. Sendo $\gamma_{q \rightarrow q}=0$, temos que

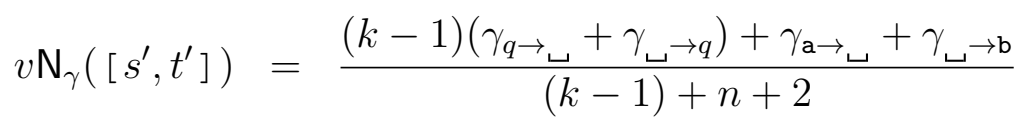

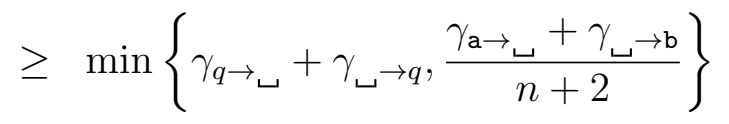

$$
\begin{aligned}
& =\frac{\gamma_{\mathrm{a} \rightarrow}+\gamma_{\lrcorner} \rightarrow \mathrm{b}}{n+2} \text {. }
\end{aligned}
$$

A Desigualdade (4.17), desde que $k-1 \geq 0$ e $n+2>0$, segue do Fato 53. Sendo

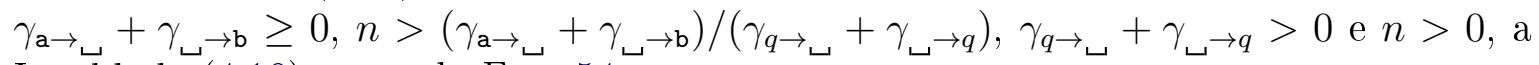
Igualdade (4.18) segue do Fato 54.

C-3 $[\mathrm{a}, q]$ alinha em $\left[s^{\prime}, t^{\prime}\right]$. 
Neste caso, $k \geq 1$. Também, desde que $\gamma_{q \rightarrow q}=0$, temos

$$
\begin{aligned}
& v \mathrm{~N}_{\gamma}\left(\left[s^{\prime}, t^{\prime}\right]\right)=\frac{(k-1)\left(\gamma_{q \rightarrow}+\gamma_{\sqcup \rightarrow q}\right)+\gamma_{\mathrm{a} \rightarrow q}+\gamma_{\sqcup \rightarrow \mathrm{b}}+\gamma_{q \rightarrow \sqcup}}{(k-1)+n+2}
\end{aligned}
$$

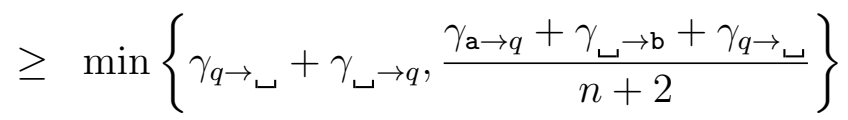

$$
\begin{aligned}
& =\frac{\gamma_{\mathrm{a} \rightarrow q}+\gamma_{\llcorner\rightarrow \mathrm{b}}+\gamma_{q \rightarrow}}{n+2} \text {. }
\end{aligned}
$$

A Desigualdade (4.19), desde que $k-1 \geq 0$ e $n+2>0$, segue do Fato 53. Sendo

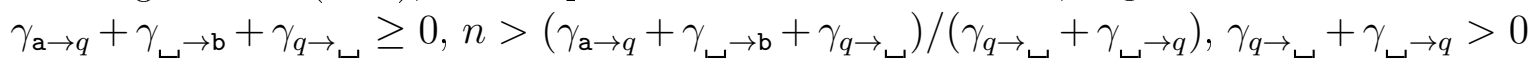
e $n>0$, a Igualdade (4.20) segue do Fato 54 .

Suponha que $\gamma_{q \rightarrow \sqcup}=\gamma_{\lrcorner \rightarrow q}$. Isto implica que

$$
\gamma_{q \rightarrow_{\sqcup}}=\max \left\{\gamma_{q \rightarrow}, \gamma_{\sqcup \rightarrow q}\right\}=Q_{\max } .
$$

Como opt $\mathrm{N}_{\gamma} \in \mathcal{P r}$, temos pelo Corolário 49 que $\gamma_{\mathrm{a} \rightarrow q} \geq 0$. Segue de (4.20) e da definição de $Q_{\max }$ que

$$
\begin{aligned}
v \mathrm{~N}_{\gamma}\left(\left[s^{\prime}, t^{\prime}\right]\right) & \geq \frac{\gamma_{\mathrm{a} \rightarrow q}+\gamma_{\sqcup \rightarrow \mathrm{b}}+\gamma_{q \rightarrow}}{n+2} \\
& \geq \frac{0+\gamma_{\sqcup \rightarrow \mathrm{b}}+Q_{\max }}{n+2} \geq \frac{\gamma_{\sqcup \rightarrow \mathrm{b}}+\gamma_{\mathrm{a} \rightarrow}}{n+2}
\end{aligned}
$$

e a prova está feita. Assumimos então que $\gamma_{q \rightarrow_{\sqcup}} \neq \gamma_{\llcorner\rightarrow q}$. Por hipótese, isto implica que $\gamma_{\mathrm{a} \rightarrow \sqcup} \leq \gamma_{\mathrm{a} \rightarrow q}+\gamma_{q \rightarrow_{\llcorner}}$. Segue de (4.20) que

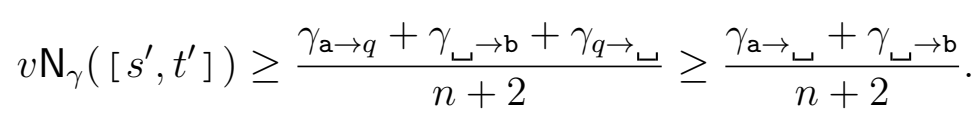

C-4 : $[q, \mathrm{~b}]$ alinha em $\left[s^{\prime}, t^{\prime}\right]$.

A prova desse caso é similar ao do Caso C-3, usando $\gamma_{\llcorner\rightarrow \mathrm{b}} \leq \gamma_{\sqcup \rightarrow q}+\gamma_{q \rightarrow \mathrm{b}}$ em vez de $\gamma_{\mathrm{a} \rightarrow\llcorner} \leq \gamma_{\mathrm{a} \rightarrow q}+\gamma_{q \rightarrow \sqcup}$ quando $\gamma_{q \rightarrow \sqcup} \neq \gamma_{\llcorner\rightarrow q}$.

Proposição 56. Sejam $o p t \mathrm{~N}_{\gamma} \in \mathcal{P r}, \mathrm{a}, \mathrm{b} \in \Sigma \mathrm{e} s, t \in \Sigma^{*}$. Se [a, b] alinha em um alinhamento N-ótimo de comprimento máximo de $(s, t)$, então

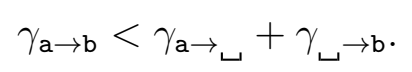

Prova. Suponha que $\left[s^{\prime}, t^{\prime}\right]$ é um alinhamento N-ótimo de comprimento máximo de $(s, t)$ e $j$ é um inteiro tal que $\left[\mathrm{a}=s^{\prime}(j), \mathrm{b}=t^{\prime}(j)\right]$ alinha em $\left[s^{\prime}, t^{\prime}\right]$. Considere o seguinte alinhamento obtido a partir de $\left[s^{\prime}, t^{\prime}\right]$

$$
A=\left[\begin{array}{cccc}
s^{\prime}(1 \ldots j-1) & \mathrm{a} & \sqcup & s^{\prime}\left(j+1 \ldots\left|s^{\prime}\right|\right) \\
t^{\prime}(1 \ldots j-1) & \sqcup & \mathrm{b} & t^{\prime}\left(j+1 \ldots\left|t^{\prime}\right|\right)
\end{array}\right] .
$$


Sendo $\left[s^{\prime}, t^{\prime}\right]$ alinhamento N-ótimo de comprimento máximo, segue que

$$
\begin{aligned}
\frac{v \mathrm{~A}_{\gamma}\left(\left[s^{\prime}, t^{\prime}\right]\right)}{\left|\left[s^{\prime}, t^{\prime}\right]\right|}=v \mathrm{~N}_{\gamma}\left(\left[s^{\prime}, t^{\prime}\right]\right) & <v \mathrm{~N}_{\gamma}(A)=\frac{v \mathrm{~A}_{\gamma}(A)}{|A|} \\
& =\frac{v \mathrm{~A}_{\gamma}\left(\left[s^{\prime}, t^{\prime}\right]\right)+\gamma_{\mathrm{a} \rightarrow \sqcup}+\gamma_{\llcorner\rightarrow \mathrm{b}}-\gamma_{\mathrm{a} \rightarrow \mathrm{b}}}{\left|\left[s^{\prime}, t^{\prime}\right]\right|+1},
\end{aligned}
$$

o que implica que

$$
\frac{v \mathrm{~A}_{\gamma}\left(\left[s^{\prime}, t^{\prime}\right]\right)}{\left|\left[s^{\prime}, t^{\prime}\right]\right|}<\gamma_{\mathrm{a} \rightarrow \sqcup}+\gamma_{\sqcup \rightarrow \mathrm{b}}-\gamma_{\mathrm{a} \rightarrow \mathrm{b}} .
$$

Como opt $\mathrm{N}_{\gamma} \in \mathcal{P r}$, temos opt $\mathrm{N}_{\gamma}(s, t) \geq 0$. Segue que

$$
0 \leq \operatorname{opt} \mathrm{N}_{\gamma}(s, t)=\frac{v \mathrm{~A}_{\gamma}\left(\left[s^{\prime}, t^{\prime}\right]\right)}{\left|\left[s^{\prime}, t^{\prime}\right]\right|}<\gamma_{\mathrm{a} \rightarrow \sqcup}+\gamma_{\iota \rightarrow \mathrm{b}}-\gamma_{\mathrm{a} \rightarrow \mathrm{b}}
$$

o que implica que $\gamma_{\mathrm{a} \rightarrow \mathrm{b}}<\gamma_{\mathrm{a} \rightarrow_{\sqcup}}+\gamma_{\boldsymbol{L}_{\rightarrow} \mathrm{b}}$. Portanto, se [a,b] alinha em um alinhamento

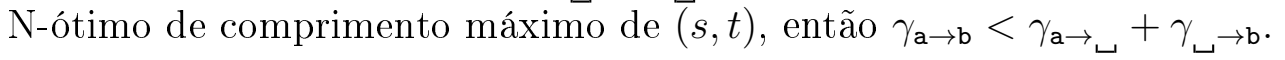

Fato 57. Sejam $x, y \in \mathbb{R}$. Se $x<y$, então

$$
\frac{x}{n+1}<\frac{y}{n+2}
$$

para todo inteiro positivo $n>(2 x-y) /(y-x)$.

Lema 58. Seja opt $\mathrm{N}_{\gamma} \in \mathcal{P r}$. Então, opt $\mathrm{N}_{\gamma}(s, t)=\operatorname{opt} \mathrm{N}_{\gamma}(t, s)$ para cada $s, t \in \Sigma^{*}$ se e somente se, para cada $\mathrm{a}, \mathrm{b} \in \Sigma$,

(i) $\gamma_{\mathrm{a} \rightarrow \sqcup}=\gamma_{\llcorner\rightarrow \mathrm{a}}, \mathrm{e}$

(ii) se $\gamma_{\mathrm{a} \rightarrow \mathrm{b}}<\gamma_{\mathrm{a} \rightarrow{ }^{-}}+\gamma_{\mathrm{b} \rightarrow \mathrm{b}}$, então $\gamma_{\mathrm{a} \rightarrow \mathrm{b}}=\gamma_{\mathrm{b} \rightarrow \mathrm{a}}$.

Prova. Para provar o lema, mostramos que se opt $\mathrm{N}_{\gamma}(s, t)=\operatorname{opt} \mathrm{N}_{\gamma}(t, s)$ para cada $s, t \in \Sigma^{*}$, então valem $(i)$ e $(i i)$ e, depois, que se valem $(i)$ e $(i i)$, então opt $\mathrm{N}_{\gamma}(s, t)=o p t \mathrm{~N}_{\gamma}(t, s)$ para cada $s, t \in \Sigma^{*}$.

Suponha que opt $\mathrm{N}_{\gamma}(s, t)=o p t \mathrm{~N}_{\gamma}(t, s)$ para cada $s, t \in \Sigma^{*}$. Sejam a, b $\in \Sigma$. Pelo Corolá-

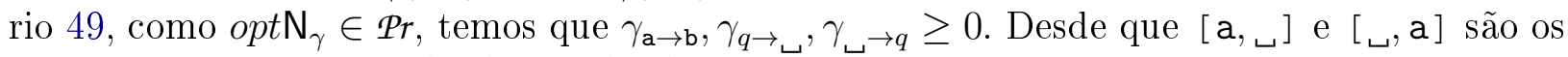
únicos alinhamentos de $(\mathrm{a}, \epsilon)$ e de $(\epsilon, \mathrm{a})$, respectivamente, segue que

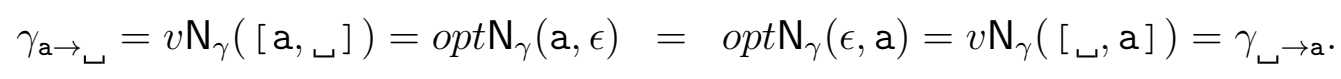

Logo, se opt $\mathrm{N}_{\gamma}(s, t)=\operatorname{opt} \mathrm{N}_{\gamma}(t, s)$ para cada $s, t \in \Sigma^{*}$, então vale $(i)$ para cada a $\in \Sigma$.

Para mostrar que vale $(i i)$, consideramos dois casos: $Q_{\max }=0$ e $Q_{\max } \neq 0$.

Suponha que $Q_{\max }=0$ e a, $\mathrm{b} \in \Sigma$. Sendo $\gamma_{\mathrm{a} \rightarrow \mathrm{b}} \geq 0$ e $Q_{\max } \geq \gamma_{\mathrm{a} \rightarrow \mathrm{b}}, \gamma_{\mathrm{b}} \rightarrow \mathrm{b}$, segue que $\gamma_{\mathrm{a} \rightarrow \mathrm{b}} \geq 0=0+0=Q_{\max }+Q_{\max } \geq \gamma_{\mathrm{a} \rightarrow_{\llcorner}}+\gamma_{\llcorner} \rightarrow \mathrm{b}$. Portanto, se $Q_{\max }=0$, então vale (ii) por vacuidade.

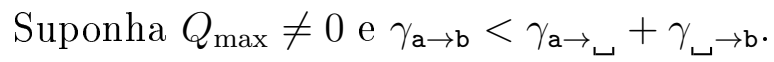

Seja

$$
n>\frac{2 \gamma_{a \rightarrow b}-\left(\gamma_{a \rightarrow \sqcup}+\gamma_{\lrcorner \rightarrow b}\right)}{\gamma_{a \rightarrow \sqcup}+\gamma_{\sqcup \rightarrow b}-\gamma_{a \rightarrow b}}
$$


um inteiro suficientemente grande satisfazendo também a Proposição 55. Desde que, por $(i)$,

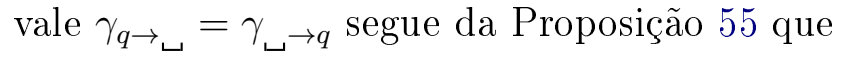

$$
\begin{aligned}
& \operatorname{opt} \mathrm{N}_{\gamma}\left(q^{n} \mathrm{a}, q^{n} \mathrm{~b}\right)=\min \left\{\frac{\gamma_{\mathrm{a} \rightarrow \mathrm{b}}}{n+1}, \frac{\gamma_{\mathrm{a} \rightarrow}+\gamma_{\sqcup} \rightarrow \mathrm{b}}{n+2}\right\} \mathrm{e} \\
& \operatorname{opt} \mathrm{N}_{\gamma}\left(q^{n} \mathrm{~b}, q^{n} \mathrm{a}\right)=\min \left\{\frac{\gamma_{\mathrm{b} \rightarrow \mathrm{a}}}{n+1}, \frac{\gamma_{\mathrm{b} \rightarrow}+\gamma_{\sqcup} \rightarrow \mathrm{a}}{n+2}\right\} .
\end{aligned}
$$

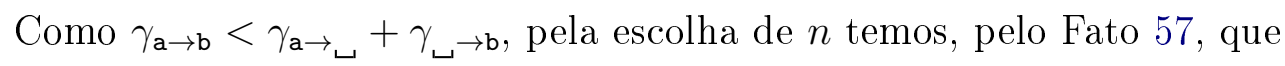

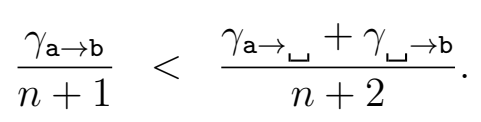

Por (4.21) e (4.23) temos que $\operatorname{opt} \mathrm{N}_{\gamma}\left(q^{n} \mathrm{a}, q^{n} \mathrm{~b}\right)=\gamma_{\mathrm{a} \rightarrow \mathrm{b}} /(n+1)$ e, sendo opt $\mathrm{N}_{\gamma}\left(q^{n} \mathrm{a}, q^{n} \mathrm{~b}\right)=$ $\operatorname{opt} \mathrm{N}_{\gamma}\left(q^{n} \mathrm{~b}, q^{n} \mathrm{a}\right)$, que

$$
\operatorname{opt} \mathrm{N}_{\gamma}\left(q^{n} \mathrm{~b}, q^{n} \mathrm{a}\right)=\operatorname{opt} \mathrm{N}_{\gamma}\left(q^{n} \mathrm{a}, q^{n} \mathrm{~b}\right)<\frac{\gamma_{\mathrm{a} \rightarrow}+\gamma_{\boldsymbol{\sqcup}} \rightarrow \mathrm{b}}{n+2} .
$$

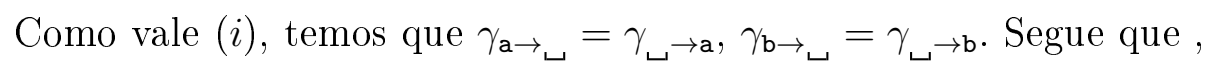

$$
\operatorname{opt} \mathrm{N}_{\gamma}\left(q^{n} \mathrm{~b}, q^{n} \mathrm{a}\right)<\frac{\gamma_{\mathrm{a} \rightarrow \sqcup}+\gamma_{\sqcup} \rightarrow \mathrm{b}}{n+2}=\frac{\gamma_{\mathrm{b} \rightarrow}+\gamma_{\llcorner} \rightarrow \mathrm{a}}{n+2},
$$

o que implica por (4.22) que opt $\mathrm{N}_{\gamma}\left(q^{n} \mathrm{~b}, q^{n} \mathrm{a}\right)=\gamma_{\mathrm{b} \rightarrow \mathrm{a}} /(n+1)$. Segue, sendo opt $\mathrm{N}_{\gamma}\left(q^{n} \mathrm{a}, q^{n} \mathrm{~b}\right)=$ $\gamma_{\mathrm{a} \rightarrow \mathrm{b}} /(n+1)$, que

$$
\frac{\gamma_{\mathrm{a} \rightarrow \mathrm{b}}}{n+1}=\operatorname{opt} \mathrm{N}_{\gamma}\left(q^{n} \mathrm{a}, q^{n} \mathrm{~b}\right)=\operatorname{opt} \mathrm{N}_{\gamma}\left(q^{n} \mathrm{~b}, q^{n} \mathrm{a}\right)=\frac{\gamma_{\mathrm{b} \rightarrow \mathrm{a}}}{n+1}
$$

o que implica que $\gamma_{\mathrm{a} \rightarrow \mathrm{b}}=\gamma_{\mathrm{b} \rightarrow \mathrm{a}}$. Portanto, se $\operatorname{opt} \mathrm{N}_{\gamma}(s, t)=\operatorname{opt} \mathrm{N}_{\gamma}(t, s)$ para cada $s, t \in \Sigma^{*}$, então também vale (ii) para cada $\mathrm{a}, \mathrm{b} \in \Sigma$.

Reciprocamente, suponha que valem $(i)$ e $(i i)$ para cada a, b $\in \Sigma$. Sejam $s, t \in \Sigma^{*}$ e $\left[s^{\prime}, t^{\prime}\right]$ um alinhamento $N$-ótimo de comprimento máximo de $(s, t)$. Se $s=\epsilon$ e $t=\epsilon$, então $s^{\prime}=\epsilon=t^{\prime}$ o que implica que

$$
\operatorname{opt} \mathrm{N}_{\gamma}(s, t)=v \mathrm{~N}_{\gamma}\left(\left[s^{\prime}, t^{\prime}\right]\right)=v \mathrm{~N}_{\gamma}([\epsilon, \epsilon])=0=v \mathrm{~N}_{\gamma}([\epsilon, \epsilon])=v \mathrm{~N}_{\gamma}\left(\left[t^{\prime}, s^{\prime}\right]\right)=\operatorname{opt} \mathrm{N}_{\gamma}(t, s) .
$$

Suponha então que $s \neq \epsilon$ ou $t \neq \epsilon$. Logo, $\left|\left[s^{\prime}, t^{\prime}\right]\right|>0$. Para cada inteiro $j$, se $s^{\prime}(j)=$ ou $t^{\prime}(j)=$, então, por $(i), \gamma_{s^{\prime}(j) \rightarrow t^{\prime}(j)}=\gamma_{t^{\prime}(j) \rightarrow s^{\prime}(j)}$; e se $s^{\prime}(j) \neq \_$e $t^{\prime}(j) \neq$ , então, sendo $\left[s^{\prime}(j), t^{\prime}(j)\right]$ alinhado e opt $\mathrm{N}_{\gamma} \in \mathcal{P r}$, temos que $\gamma_{s^{\prime}(j) \rightarrow t^{\prime}(j)}<\gamma_{s^{\prime}(j) \rightarrow \sqcup}+\gamma_{\iota \rightarrow t^{\prime}(j)}$ pela Proposição 56, o que implica por $(i i)$ que $\gamma_{s^{\prime}(j) \rightarrow t^{\prime}(j)}=\gamma_{t^{\prime}(j) \rightarrow s^{\prime}(j)}$. Usando esses argumentos, sendo $\left|s^{\prime}, t^{\prime}\right|>0$, temos que

$$
\begin{aligned}
\operatorname{opt} \mathrm{N}_{\gamma}(s, t) & =v \mathrm{~N}_{\gamma}\left(\left[s^{\prime}, t^{\prime}\right]\right)=\frac{\sum_{j} \gamma_{s^{\prime}(j) \rightarrow t^{\prime}(j)}}{\left|\left[s^{\prime}, t^{\prime}\right]\right|} \\
& =\frac{\sum_{j} \gamma_{t^{\prime}(j) \rightarrow s^{\prime}(j)}}{\left|\left[s^{\prime}, t^{\prime}\right]\right|}=v \mathrm{~N}_{\gamma}\left(\left[t^{\prime}, s^{\prime}\right]\right) \geq \operatorname{opt} \mathrm{N}_{\gamma}(t, s) .
\end{aligned}
$$

Similarmente, temos que opt $\mathrm{N}_{\gamma}(s, t) \leq o p t \mathrm{~N}_{\gamma}(t, s)$. Segue que opt $\mathrm{N}_{\gamma}(s, t)=\operatorname{opt} \mathrm{N}_{\gamma}(t, s)$.

Portanto, se valem $(i)$ e $(i i)$ para cada $\mathrm{a}, \mathrm{b} \in \Sigma$, então $\operatorname{opt} \mathrm{N}_{\gamma}(s, t)=\operatorname{opt} \mathrm{N}_{\gamma}(t, s)$ para cada 
$s, t \in \Sigma^{*}$.

Proposição 59. Sejam opt $\mathrm{N}_{\gamma} \in \mathcal{P r}$ e a, b $\in \Sigma$. Se $\operatorname{opt} \mathrm{N}_{\gamma}(s, u) \leq o p t \mathrm{~N}_{\gamma}(s, t)+o p t \mathrm{~N}_{\gamma}(t, u)$ para cada $s, t, u \in \Sigma^{*}$, então

$$
\gamma_{\mathrm{a} \rightarrow \sqcup} \leq \gamma_{\mathrm{a} \rightarrow \mathrm{b}}+\gamma_{\mathrm{b} \rightarrow \sqcup} \quad \text { e } \quad \gamma_{\sqcup \rightarrow \mathrm{a}} \leq \gamma_{\sqcup \rightarrow \mathrm{b}}+\gamma_{\mathrm{b} \rightarrow \mathrm{a}}
$$

Prova. Suponha que opt $\mathrm{N}_{\gamma}(s, u) \leq o p t \mathrm{~N}_{\gamma}(s, t)+o p t \mathrm{~N}_{\gamma}(t, u)$ para cada $s, t, u \in \Sigma^{*}$. Como $\left[\mathrm{a},{ }_{\longleftarrow}\right]$ é o único alinhamento de $(\mathrm{a}, \epsilon)$, e $[\mathrm{a}, \mathrm{b}]$ e $\left[\mathrm{b},{ }_{\longleftarrow}\right]$ são alinhamentos de $(\mathrm{a}, \mathrm{b})$ e de $(\mathrm{b}, \epsilon)$ respectivamente, segue que

$$
\begin{aligned}
& \gamma_{\mathrm{a} \rightarrow \sqcup}=v \mathrm{~N}_{\gamma}\left(\left[\mathrm{a},{ }_{\sqcup}\right]\right)=\operatorname{opt} \mathrm{N}_{\gamma}(\mathrm{a}, \epsilon) \leq \operatorname{opt} \mathrm{N}_{\gamma}(\mathrm{a}, \mathrm{b})+\operatorname{opt} \mathrm{N}_{\gamma}(\mathrm{b}, \epsilon) \\
& \leq v \mathrm{~N}_{\gamma}([\mathrm{a}, \mathrm{b}])+v \mathrm{~N}_{\gamma}([\mathrm{b}, \sqcup]) \\
& =\gamma_{\mathrm{a} \rightarrow \mathrm{b}}+\gamma_{\mathrm{b} \rightarrow} \text {. }
\end{aligned}
$$

Portanto, $\gamma_{\mathrm{a} \rightarrow \iota} \leq \gamma_{\mathrm{a} \rightarrow \mathrm{b}}+\gamma_{\mathrm{b} \rightarrow}$.

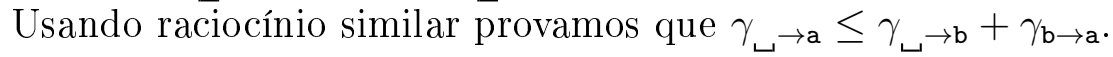

Proposição 60. Sejam opt $\mathrm{N}_{\gamma} \in \mathcal{P r}$ e a, b $\in \Sigma$. Se opt $\mathrm{N}_{\gamma}(s, u) \leq o p t \mathrm{~N}_{\gamma}(s, t)+o p t \mathrm{~N}_{\gamma}(t, u)$ para cada $s, t, u \in \Sigma^{*}$, então

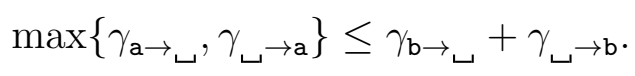

Prova. Ponha $\mathcal{G}=\max \left\{\gamma_{\mathrm{a} \rightarrow}, \gamma_{\lrcorner \rightarrow \mathrm{a}}\right\}$ e suponha que opt $\mathrm{N}_{\gamma}(s, u) \leq \operatorname{opt} \mathrm{N}_{\gamma}(s, t)+\operatorname{opt} \mathrm{N}_{\gamma}(t, u)$

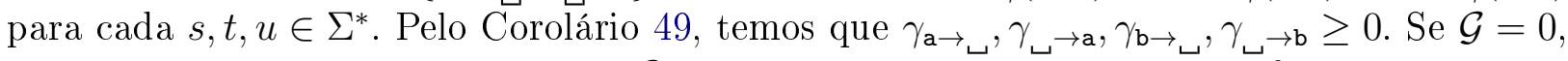

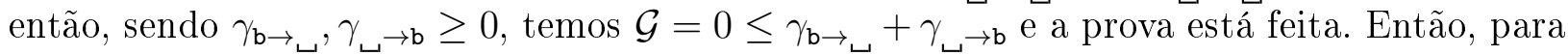

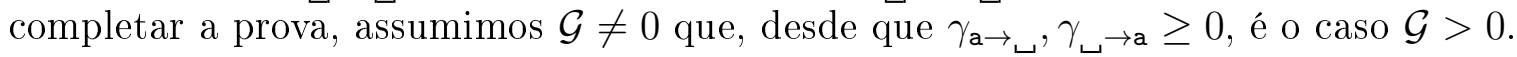

Suponha, por contradição, que $\mathcal{G}>\gamma_{\mathrm{b} \rightarrow}+\gamma_{\llcorner} \rightarrow \mathrm{b}$. Seja um inteiro positivo

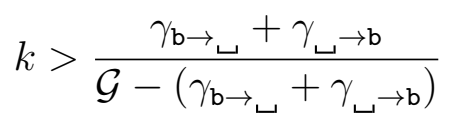

e suponha $\mathcal{G}=\gamma_{\mathrm{a} \rightarrow \mathrm{w}}$.

Como $\gamma_{a \rightarrow \sqcup}, \gamma_{\iota \rightarrow a} \geq 0$ e $\gamma_{a \rightarrow \sqcup}=\mathcal{G}>0$, temos que $\gamma_{a \rightarrow_{\sqcup}}+\gamma_{\sqcup \rightarrow a}>0$. Desde que opt $\mathrm{N}_{\gamma} \in \mathcal{P r}$, segue do Corolário 49 que $\gamma_{\mathrm{a} \rightarrow \mathrm{a}}=0$. Logo,

$$
\operatorname{opt} \mathrm{N}_{\gamma}\left(\mathrm{a}^{k}, \mathrm{a}^{k} \mathrm{~b}\right) \leq v \mathrm{~N}_{\gamma}\left(\left[\begin{array}{cc}
\mathrm{a}^{k} & \sqcup \\
\mathrm{a}^{k} & \mathrm{~b}
\end{array}\right]\right)=\frac{\gamma_{\lrcorner \rightarrow \mathrm{b}}}{k+1}
$$

Note que

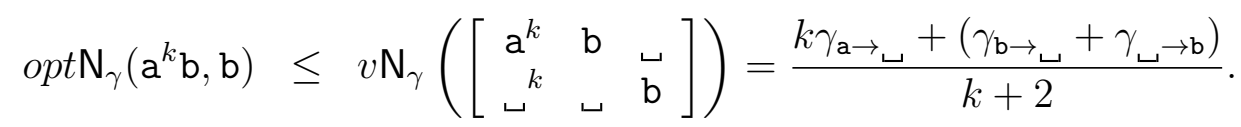

Em qualquer alinhamento de $\left(\mathrm{a}^{k}, \mathrm{~b}\right)$, ou $[\mathrm{a}, \mathrm{b}]$ alinha ou $\left[\_, \mathrm{b}\right]$ alinha. Então,

$$
\begin{aligned}
\operatorname{opt}_{\gamma}\left(\mathrm{a}^{k}, \mathrm{~b}\right) & =\min \left\{v \mathrm{~N}_{\gamma}\left(\left[\begin{array}{cc}
\mathrm{a}^{k-1} & \mathrm{a} \\
k-1 & \mathrm{~b}
\end{array}\right]\right), v \mathrm{~N}_{\gamma}\left(\left[\begin{array}{cc}
\mathrm{a}^{k} & \sqcup \\
k & \mathrm{~b}
\end{array}\right]\right)\right\} \\
& =\min \left\{\frac{(k-1) \gamma_{\mathrm{a} \rightarrow \sqcup}+\gamma_{\mathrm{a} \rightarrow \mathrm{b}}}{k}, \frac{k \gamma_{\mathrm{a} \rightarrow \sqcup}+\gamma_{\sqcup} \rightarrow \mathrm{b}}{k+1}\right\} .
\end{aligned}
$$


Suponha que

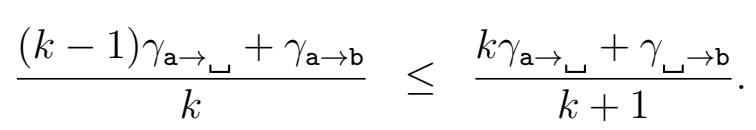

Como uma consequência de $\gamma_{\mathrm{a} \rightarrow \sqcup} \leq \gamma_{\mathrm{a} \rightarrow \mathrm{b}}+\gamma_{\mathrm{b} \rightarrow_{\sqcup}}$ (Proposição 59) e sendo $k>0$, temos que

$$
\frac{k \gamma_{\mathrm{a} \rightarrow_{\sqcup}}-\gamma_{\mathrm{b} \rightarrow_{\sqcup}}}{k} \leq \frac{(k-1) \gamma_{\mathrm{a} \rightarrow_{\sqcup}}+\gamma_{\mathrm{a} \rightarrow \mathrm{b}}}{k}
$$

Segue que

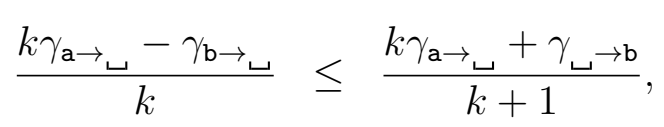

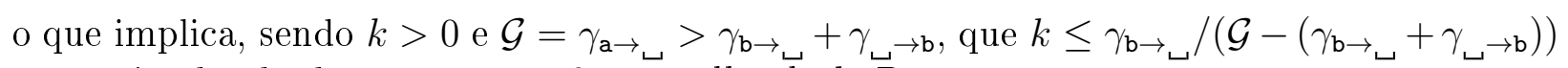
contrariando, desde que $\gamma_{\lrcorner \rightarrow \mathrm{b}} \geq 0$, a escolha de $k$. Portanto,

$$
\frac{(k-1) \gamma_{\mathrm{a} \rightarrow \sqcup}+\gamma_{\mathrm{a} \rightarrow \mathrm{b}}}{k} \not \leq \frac{k \gamma_{\mathrm{a} \rightarrow \sqcup}+\gamma_{\llcorner\rightarrow \mathrm{b}}}{k+1},
$$

o que implica por (4.26) que

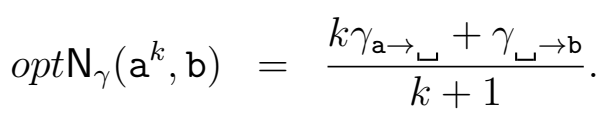

Como $\operatorname{opt} \mathrm{N}_{\gamma}\left(\mathrm{a}^{k}, \mathrm{~b}\right) \leq \operatorname{opt} \mathrm{N}_{\gamma}\left(\mathrm{a}^{k}, \mathrm{a}^{k} \mathrm{~b}\right)+\operatorname{opt} \mathrm{N}_{\gamma}\left(\mathrm{a}^{k} \mathrm{~b}, \mathrm{~b}\right)$, temos por (4.24), (4.25) e (4.27) que

$$
\begin{aligned}
\frac{k \gamma_{\mathrm{a} \rightarrow \sqcup}+\gamma_{\sqcup \rightarrow \mathrm{b}}}{k+1}=\operatorname{opt} \mathrm{N}_{\gamma}\left(\mathrm{a}^{k}, \mathrm{~b}\right) & \leq \operatorname{opt} \mathrm{N}_{\gamma}\left(\mathrm{a}^{k}, \mathrm{a}^{k} \mathrm{~b}\right)+\operatorname{opt} \mathrm{N}_{\gamma}\left(\mathrm{a}^{k} \mathrm{~b}, \mathrm{~b}\right) \\
& \leq \frac{\gamma_{\sqcup \rightarrow \mathrm{b}}}{k+1}+\frac{k \gamma_{\mathrm{a} \rightarrow \sqcup}+\left(\gamma_{\mathrm{b} \rightarrow \sqcup}+\gamma_{\sqcup \rightarrow \mathrm{b}}\right)}{k+2}
\end{aligned}
$$

o que implica, sendo $k>0$ e $\gamma_{\mathrm{a} \rightarrow_{\sqcup}}-\left(\gamma_{\mathrm{b} \rightarrow_{\sqcup}}+\gamma_{\iota^{\rightarrow b}}\right)>0$, que

$$
k \leq \frac{\gamma_{\mathrm{b} \rightarrow \sqcup}+\gamma_{\sqcup \rightarrow \mathrm{b}}}{\gamma_{\mathrm{a} \rightarrow \sqcup}-\left(\gamma_{\mathrm{b} \rightarrow \sqcup}+\gamma_{\sqcup \rightarrow \mathrm{b}}\right)},
$$

contrariando novamente a escolha de $k$. Portanto, $\mathcal{G}=\gamma_{\iota_{\rightarrow}}$. Entretanto, usando os mesmos argumentos e os pares de sequências $\left(\mathrm{b}, \mathrm{a}^{k}\right),\left(\mathrm{b}, \mathrm{a}^{k} \mathrm{~b}\right)$ e $\left(\mathrm{a}^{k} \mathrm{~b}, \mathrm{a}^{k}\right)$, obtemos contradição novamente. Portanto, se opt $\mathrm{N}_{\gamma}(s, u) \leq o p t \mathrm{~N}_{\gamma}(s, t)+o p t \mathrm{~N}_{\gamma}(t, u)$ para cada $s, t, u \in \Sigma^{*}$, então

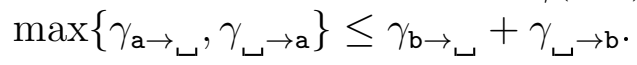

Proposição 61. Sejam opt $\mathrm{N}_{\gamma} \in \operatorname{Pr}$ e a, b, c $\in \Sigma$. Se opt $\mathrm{N}_{\gamma}(s, t) \leq o p t \mathrm{~N}_{\gamma}(s, u)+o p t \mathrm{~N}_{\gamma}(u, t)$ para cada $s, t, u \in \Sigma^{*}$, então

$$
\min \left\{\gamma_{\mathrm{a} \rightarrow \mathrm{c}}, \gamma_{\mathrm{a} \rightarrow \sqcup}+\gamma_{\lrcorner \rightarrow \mathrm{c}}\right\} \leq \gamma_{\mathrm{a} \rightarrow \mathrm{b}}+\gamma_{\mathrm{b} \rightarrow \mathrm{c}}
$$

Prova. Como opt $\mathrm{N}_{\gamma} \in \mathcal{P} r$, pelo Corolário 49 temos que $\gamma_{\mathrm{a} \rightarrow \mathrm{c}}, \gamma_{\mathrm{a} \rightarrow \mathrm{b}}, \gamma_{\mathrm{b} \rightarrow \mathrm{c}} \geq 0$.

Se $Q_{\max }=0$, então, como opt $\mathrm{N}_{\gamma} \in \mathcal{P} r$, pela Proposição 52 temos que $\gamma_{\mathrm{a} \rightarrow \sqcup}=\gamma_{\iota^{\rightarrow a}}=0$ e segue, sendo $\gamma_{\mathrm{a} \rightarrow \mathrm{c}}, \gamma_{\mathrm{a} \rightarrow \mathrm{b}}, \gamma_{\mathrm{b} \rightarrow \mathrm{c}} \geq 0$, que $\min \left\{\gamma_{\mathrm{a} \rightarrow \mathrm{c}}, \gamma_{\mathrm{a} \rightarrow_{\mathrm{b}}}+\gamma_{\mathrm{c} \rightarrow \mathrm{c}}\right\}=0 \leq \gamma_{\mathrm{a} \rightarrow \mathrm{b}}+\gamma_{\mathrm{b} \rightarrow \mathrm{c}}$ e a proposição está provada por vacuidade. Assumimos então que $Q_{\max } \neq 0$. 
Suponha que opt $\mathrm{N}_{\gamma}(s, t) \leq \operatorname{opt} \mathrm{N}_{\gamma}(s, u)+o p t \mathrm{~N}_{\gamma}(u, t)$ para cada $s, t, u \in \Sigma^{*}$. Segue da

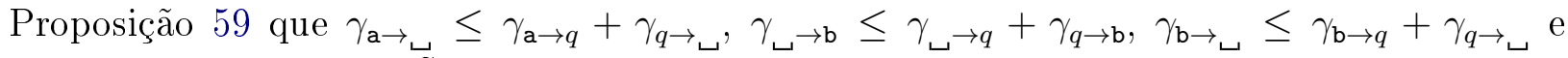

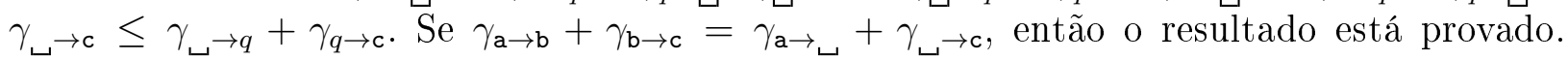
Assumimos então que $\gamma_{\mathrm{a} \rightarrow \mathrm{b}}+\gamma_{\mathrm{b} \rightarrow \mathrm{c}} \neq \gamma_{\mathrm{a} \rightarrow \mathrm{b}}+\gamma_{\mathrm{c} \rightarrow \mathrm{c}}$. Sendo $Q_{\max } \neq 0$, segue da Proposição 55

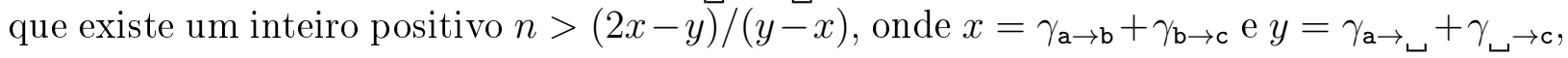
tal que

$$
\begin{aligned}
\min \left\{\frac{\gamma_{\mathrm{a} \rightarrow \mathrm{c}}}{n+1}, \frac{\gamma_{\mathrm{a} \rightarrow}+\gamma_{\llcorner} \rightarrow \mathrm{c}}{n+2}\right\} & =\operatorname{opt} \mathrm{N}_{\gamma}\left(q^{n} \mathrm{a}, q^{n} \mathrm{c}\right) \\
\operatorname{opt} \mathrm{N}_{\gamma}\left(q^{n} \mathrm{a}, q^{n} \mathrm{~b}\right) & \leq \frac{\gamma_{\mathrm{a} \rightarrow \mathrm{b}}}{n+1} \\
\operatorname{opt} \mathrm{N}_{\gamma}\left(q^{n} \mathrm{~b}, q^{n} \mathrm{c}\right) & \leq \frac{\gamma_{\mathrm{b} \rightarrow \mathrm{c}}}{n+1}
\end{aligned}
$$

Segue daí que

$$
\begin{aligned}
\min \left\{\frac{\gamma_{\mathrm{a} \rightarrow \mathrm{c}}}{n+1}, \frac{\gamma_{\mathrm{a} \rightarrow \sqcup}+\gamma_{\llcorner\rightarrow \mathrm{c}}}{n+2}\right\} & =\operatorname{opt} \mathrm{N}_{\gamma}\left(q^{n} \mathrm{a}, q^{n} \mathrm{c}\right) \\
& \leq \operatorname{opt} \mathrm{N}_{\gamma}\left(q^{n} \mathrm{a}, q^{n} \mathrm{~b}\right)+\operatorname{opt} \mathrm{N}_{\gamma}\left(q^{n} \mathrm{~b}, q^{n} \mathrm{c}\right) \\
& \leq \frac{\gamma_{\mathrm{a} \rightarrow \mathrm{b}}}{n+1}+\frac{\gamma_{\mathrm{b} \rightarrow \mathrm{c}}}{n+1} .
\end{aligned}
$$

Se

$$
\frac{\gamma_{\mathrm{a} \rightarrow \mathrm{c}}}{n+1}=\min \left\{\frac{\gamma_{\mathrm{a} \rightarrow \mathrm{c}}}{n+1}, \frac{\gamma_{\mathrm{a} \rightarrow}+\gamma_{\llcorner} \rightarrow \mathrm{c}}{n+2}\right\} \leq \frac{\gamma_{\mathrm{a} \rightarrow \mathrm{b}}}{n+1}+\frac{\gamma_{\mathrm{b} \rightarrow \mathrm{c}}}{n+1},
$$

então, sendo $n>0$, temos que $\gamma_{\mathrm{a} \rightarrow \mathrm{c}} \leq \gamma_{\mathrm{a} \rightarrow \mathrm{b}}+\gamma_{\mathrm{b} \rightarrow \mathrm{c}}$ e a prova está feita, e se

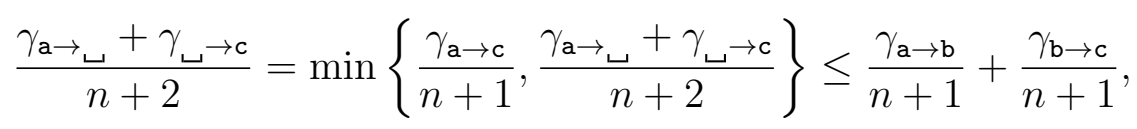

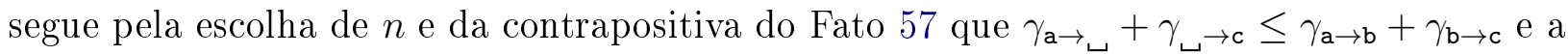
prova também está feita.

Lema 62. Sejam opt $\mathrm{N}_{\gamma} \in \mathcal{P r}$. Então, opt $\mathrm{N}_{\gamma}(s, u) \leq \operatorname{opt} \mathrm{N}_{\gamma}(s, t)+\operatorname{opt} \mathrm{N}_{\gamma}(t, u)$ para cada $s, t, u \in \Sigma^{*}$ se e somente se

(i) $\gamma_{\mathrm{a} \rightarrow_{\sqcup}} \leq \gamma_{\mathrm{a} \rightarrow \mathrm{b}}+\gamma_{\mathrm{b} \rightarrow_{\sqcup}}$,

(ii) $\gamma_{\mathrm{S} \rightarrow \mathrm{a}} \leq \gamma_{\boldsymbol{S} \rightarrow \mathrm{b}}+\gamma_{\mathrm{b} \rightarrow \mathrm{a}}$,

(iii) $\min \left\{\gamma_{\mathrm{a} \rightarrow \mathrm{c}}, \gamma_{\mathrm{a} \rightarrow \sqcup}+\gamma_{\sqcup \rightarrow \mathrm{c}}\right\} \leq \gamma_{\mathrm{a} \rightarrow \mathrm{b}}+\gamma_{\mathrm{b} \rightarrow \mathrm{c}}$ e

(iv) $\max \left\{\gamma_{\mathrm{a} \rightarrow \sqcup}, \gamma_{\sqcup \rightarrow \mathrm{a}}\right\} \leq \gamma_{\mathrm{b} \rightarrow \sqcup}+\gamma_{\sqcup \rightarrow \mathrm{b}}$.

para cada a, b, c $\in \Sigma$.

Prova. Para provar o lema, vamos mostrar que se opt $\mathrm{N}_{\gamma}(s, t) \leq \operatorname{opt} \mathrm{N}_{\gamma}(s, u)+\operatorname{opt} \mathrm{N}_{\gamma}(u, t)$ para cada $s, t, u \in \Sigma^{*}$, então valem $(i),(i i)$, (iii) e (iv), e que se valem $(i),(i i)$, (iii) e (iv), então opt $\mathrm{N}_{\gamma}(s, t) \leq o p t \mathrm{~N}_{\gamma}(s, u)+o p t \mathrm{~N}_{\gamma}(u, t)$ para cada $s, t, u \in \Sigma^{*}$.

Suponha que opt $\mathrm{N}_{\gamma}(s, t) \leq$ opt $\mathrm{N}_{\gamma}(s, u)+$ opt $\mathrm{N}_{\gamma}(u, t)$ para cada $s, t, u \in \Sigma^{*}$. Sendo opt $\mathrm{N}_{\gamma} \in$ $\mathcal{P}_{r}$, seguem das Proposições 59, 60 e 61 que valem $(i),($ ii $)$, (iii) e (iv) para cada a, b, c $\in \Sigma$. Portanto, se opt $\mathrm{N}_{\gamma}(s, t) \leq o p t \mathrm{~N}_{\gamma}(s, u)+o p t \mathrm{~N}_{\gamma}(u, t)$ para cada $s, t, u \in \Sigma^{*}$, então valem $(i)$, (ii), (iii) e $(i v)$. 
Reciprocamente, suponha que valem $(i),(i i),(i i i)$ e $(i v)$ para cada a, b, c $\in \Sigma$. Sejam $s, t, u \in \Sigma^{*}$ e $A, B$ alinhamentos N-ótimos de $(s, t)$ e de $(t, u)$ respectivamente. Segue da

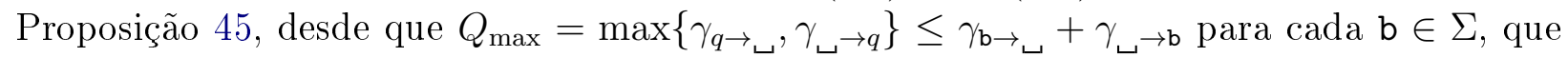
existem um inteiro $k \geq 0$ e um alinhamento $C$ de $(s, u)$ tais que

$$
v \mathrm{~A}_{\gamma}(A)+v \mathrm{~A}_{\gamma}(B) \geq v \mathrm{~A}_{\gamma}(C)+k Q_{\max }, \quad|A| \leq|C|+k, \quad|B| \leq|C|+k .
$$

Sendo opt $\mathrm{N}_{\gamma} \in \mathcal{P r}$, segue que opt $\mathrm{N}_{\gamma}(s, t) \geq 0$. Logo, $v \mathrm{~A}_{\gamma}(A) /|A|=\operatorname{opt} \mathrm{N}_{\gamma}(s, t) \geq 0$ o que implica sendo $|A|>0$ que $v \mathrm{~A}_{\gamma}(A) \geq 0$. Usando o mesmo argumento para o alinhamento $B$, temos que $v \mathrm{~A}_{\gamma}(B) \geq 0$. Segue que

$$
\begin{aligned}
\frac{v \mathrm{~A}_{\gamma}(C)+k Q_{\max }}{|C|+k} & \leq \frac{v \mathrm{~A}_{\gamma}(A)+v \mathrm{~A}_{\gamma}(B)}{|C|+k}=\frac{v \mathrm{~A}_{\gamma}(A)}{|C|+k}+\frac{v \mathrm{~A}_{\gamma}(B)}{|C|+k} \\
& \leq \frac{v \mathrm{~A}_{\gamma}(A)}{|A|}+\frac{v \mathrm{~A}_{\gamma}(B)}{|B|}=o p t \mathrm{~N}_{\gamma}(s, t)+o p t \mathrm{~N}_{\gamma}(t, u)
\end{aligned}
$$

Portanto, para mostrar que opt $\mathrm{N}_{\gamma}(s, t) \leq o p t \mathrm{~N}_{\gamma}(s, u)+o p t \mathrm{~N}_{\gamma}(u, t)$ basta provar que

$$
\frac{v \mathrm{~A}_{\gamma}(C)+k Q_{\max }}{|C|+k} \geq \operatorname{opt} \mathrm{N}_{\gamma}(s, u) .
$$

Considere dois casos: se $\left(v \mathrm{~A}_{\gamma}(C)+k Q_{\max }\right) /(|C|+k) \geq v \mathrm{~A}_{\gamma}(C) /|C|$, então sendo $C$ um alinhamento de $(s, u)$, temos

$$
\frac{v \mathrm{~A}_{\gamma}(C)+k Q_{\max }}{|C|+k} \geq \frac{v \mathrm{~A}_{\gamma}(C)}{|C|} \geq \operatorname{opt} \mathrm{N}_{\gamma}(s, u)
$$

e a prova está feita. E se $\left(v \mathrm{~A}_{\gamma}(C)+k Q_{\max }\right) /(|C|+k)<v \mathrm{~A}_{\gamma}(C) /|C|$, sendo $k \geq 0$ e $|C|>0$, temos que $v \mathrm{~A}_{\gamma}(C)>|C| Q_{\max }$. Desde que, pela Proposição 51 , opt $\mathrm{N}_{\gamma}(s, u) \leq Q_{\max }$, segue que

$$
\frac{v \mathrm{~A}_{\gamma}(C)+k Q_{\max }}{|C|+k}>\frac{|C| Q_{\max }+k Q_{\max }}{|C|+k}=Q_{\max } \geq \operatorname{opt} \mathrm{N}_{\gamma}(s, u) .
$$

Portanto, se valem (i), (ii), (iii) e (iv) para cada a, b, c $\in \Sigma$, então $\operatorname{opt} \mathrm{N}_{\gamma}(s, u) \leq$ $o p t \mathrm{~N}_{\gamma}(s, t)+o p t \mathrm{~N}_{\gamma}(t, u)$ para cada $s, t, u \in \Sigma^{*}$.

Usando os resultados obtidos nesta seção, podemos demonstrar o seguinte teorema.

Teorema 63. Seja $\gamma$ matriz de pontuação. Então, opt $\mathrm{N}_{\gamma} \in \mathcal{M}$ se e somente se $\gamma \in \mathbb{M}^{\mathbb{N}}$.

Prova. Suponha que opt $\mathrm{N}_{\gamma} \in \mathcal{M}$. Segue que opt $\mathrm{N}_{\gamma}(s, s)=0, \operatorname{opt} \mathrm{N}_{\gamma}(s, t)>0, \operatorname{opt} \mathrm{N}_{\gamma}(s, t)=$ $o p t \mathrm{~N}_{\gamma}(t, s)$ e opt $\mathrm{N}_{\gamma}(s, u) \leq \operatorname{opt} \mathrm{N}_{\gamma}(s, t)+\operatorname{opt} \mathrm{N}_{\gamma}(t, u)$ para cada $s, t, u \in \Sigma^{*}, s \neq t$. Como opt $\mathrm{N}_{\gamma}(s, s)=0$ e opt $\mathrm{N}_{\gamma}(s, t)>0$ para cada $s \neq t$, temos por definição que opt $\mathrm{N}_{\gamma} \in \operatorname{Pr}$. Sejam a, b, c símbolos arbitrários e distintos em $\Sigma$. Pelo Lema 50 temos que $\gamma_{a \rightarrow \sqcup}, \gamma_{\lrcorner \rightarrow a}, \gamma_{a \rightarrow b}>0$. Desde que $\gamma_{a \rightarrow}, \gamma_{u_{\rightarrow a}}>0$, segue do Corolário 49 que $\gamma_{a \rightarrow a}=0$. Pelo Lema 58 temos que

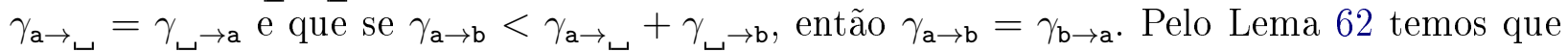
$\gamma_{a \rightarrow_{\lrcorner}} \leq \gamma_{a \rightarrow b}+\gamma_{b \rightarrow_{\lrcorner}}, \min \left\{\gamma_{a \rightarrow c}, \gamma_{a \rightarrow \sqcup}+\gamma_{\lrcorner \rightarrow c}\right\} \leq \gamma_{a \rightarrow b}+\gamma_{b \rightarrow c}$ e que $\max \left\{\gamma_{a \rightarrow}, \gamma_{\lrcorner \rightarrow a}\right\} \leq$

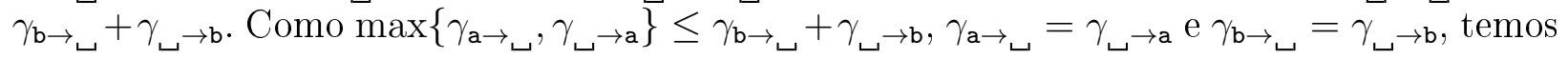
que

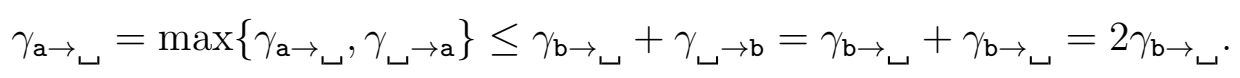

Desde que os resultados deste parágrafo valem para símbolos a, b, c $\in \Sigma$ arbitrários, segue 
que se opt $\mathrm{N}_{\gamma} \in \mathcal{M}$, então $\gamma \in \mathbb{M}^{\mathbb{N}}$.

Reciprocamente, suponha que $\gamma \in \mathbb{M}^{\mathbb{N}}$. Logo, $\gamma \in \mathbb{M}^{\mathrm{A}}$ e que $\gamma_{\mathrm{a} \rightarrow_{\sqcup}} \leq 2 \gamma_{\mathrm{b} \rightarrow_{\sqcup}}$ para cada $\mathrm{a}, \mathrm{b} \in \Sigma$. Como $\gamma \in \mathbb{M}^{\mathrm{A}}$, temos por definição

- $\gamma_{\mathrm{a} \rightarrow\llcorner}=\gamma_{\llcorner\rightarrow \mathrm{a}}>0$,

- $\gamma_{a \rightarrow b}>0$ se $a \neq b$, e $\gamma_{a \rightarrow b}=0$ se $a=b$,

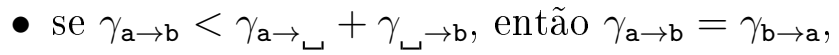

- $\gamma_{\mathrm{a} \rightarrow \sqcup} \leq \gamma_{\mathrm{a} \rightarrow \mathrm{b}}+\gamma_{\mathrm{b} \rightarrow_{\sqcup}} \mathrm{e}$

- $\min \left\{\gamma_{\mathrm{a} \rightarrow \mathrm{c}}, \gamma_{\mathrm{a} \rightarrow \boldsymbol{b}}+\gamma_{\mathrm{c} \rightarrow \mathrm{c}}\right\} \leq \gamma_{\mathrm{a} \rightarrow \mathrm{b}}+\gamma_{\mathrm{b} \rightarrow \mathrm{c}}$

para cada a, b, c $\in \Sigma$. Segue do Teorema 47 que opt $\mathrm{A}_{\gamma} \in \mathcal{M}$ e, sendo $\mathcal{M} \subseteq \mathcal{P r}$, temos que optA $\mathrm{A}_{\gamma} \in \operatorname{Pr}$. Segue do Lema 48 que opt $\mathrm{N}_{\gamma} \in \operatorname{Pr}$. Sejam $s, t, u \in \Sigma^{*}$ sequências arbitrárias, $s \neq t$. Para provar que opt $\mathrm{N}_{\gamma} \in \mathcal{M}$, basta mostrar que opt $\mathrm{N}_{\gamma}(s, s)=0, \operatorname{opt} \mathrm{N}_{\gamma}(s, t)>0$, $o p t \mathrm{~N}_{\gamma}(s, t)=o p t \mathrm{~N}_{\gamma}(t, s)$ e opt $\mathrm{N}_{\gamma}(s, u) \leq o p t \mathrm{~N}_{\gamma}(s, t)+o p t \mathrm{~N}_{\gamma}(t, u)$.

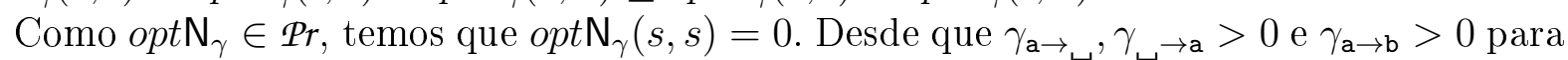

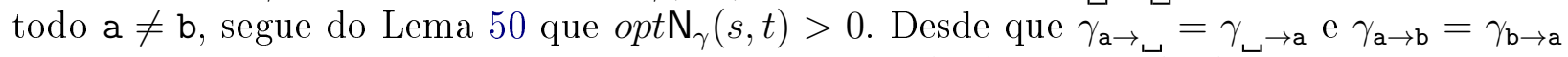
se $\gamma_{\mathrm{a} \rightarrow \mathrm{b}}<\gamma_{\mathrm{a} \rightarrow\llcorner}+\gamma_{\lrcorner \rightarrow \mathrm{b}}$, segue do Lema 58 que opt $\mathrm{N}_{\gamma}(s, t)=\operatorname{opt} \mathrm{N}_{\gamma}(t, s)$.

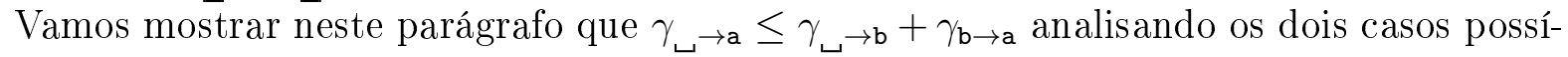

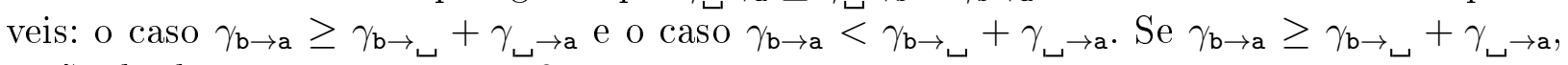

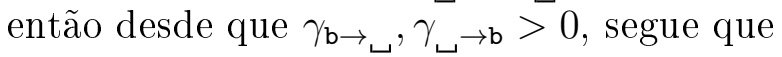

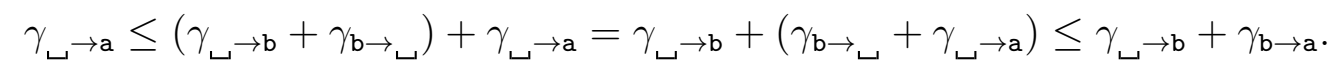

Se $\gamma_{\mathrm{b} \rightarrow \mathrm{a}}<\gamma_{\mathrm{b} \rightarrow \sqcup}+\gamma_{\iota^{\rightarrow a}}$, então segue da definição de $\mathbb{M}^{\mathrm{A}}$ que

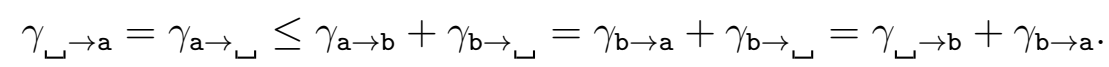

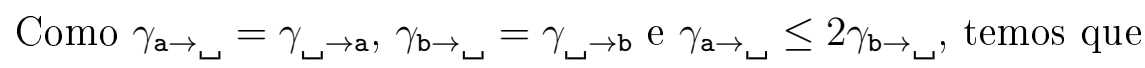

$$
\max \left\{\gamma_{\mathrm{a} \rightarrow \sqcup}, \gamma_{\sqcup \rightarrow \mathrm{a}}\right\}=\gamma_{\mathrm{a} \rightarrow \sqcup} \leq 2 \gamma_{\mathrm{b} \rightarrow \sqcup}=\gamma_{\mathrm{b} \rightarrow \sqcup}+\gamma_{\llcorner\rightarrow \mathrm{b}} \text {. }
$$

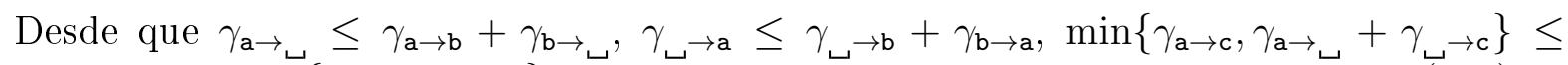
$\gamma_{\mathrm{a} \rightarrow \mathrm{b}}+\gamma_{\mathrm{b} \rightarrow \mathrm{c}}$ e $\max \left\{\gamma_{\mathrm{a} \rightarrow \sqcup}, \gamma_{\lrcorner \rightarrow \mathrm{a}}\right\} \leq \gamma_{\mathrm{b} \rightarrow \sqcup}+\gamma_{\llcorner\rightarrow \mathrm{b}}$, segue do Lema 62 que $\operatorname{optN}_{\gamma}(s, u) \leq$ $o p t \mathrm{~N}_{\gamma}(s, t)+o p t \mathrm{~N}_{\gamma}(t, u)$.

\subsection{Distância de edição}

Nesta seção verificamos quais são as condições suficientes e necessárias para que cada uma das propriedades de uma métrica sejam satisfeitas por opt $\mathrm{E}_{\gamma}$. Vamos mostrar, por exemplo, que

$$
\begin{aligned}
& \begin{array}{c|cccc} 
& \mathrm{a} & \mathrm{b} & \mathrm{c} & \mathrm{c} \\
\hline \mathrm{a} & 2 & 3 & 4 & 10
\end{array}
\end{aligned}
$$

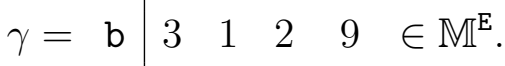

$$
\begin{aligned}
& \text { c } 4 \begin{array}{llll}
4 & 2 & 0 & 5
\end{array} \\
& \begin{array}{l|lll} 
& 9 & 8 & 5
\end{array}
\end{aligned}
$$

Lema 64. Seja $\gamma$ uma matriz de pontuação. Se $D(\gamma)$ possui um ciclo negativo, então opt $\mathrm{E}_{\gamma}(s, t)$ é indefinido para quaisquer $s, t \in \Sigma^{*}$. 
Prova. Sejam $s, t \in \Sigma^{*}$ e $C=\left(a_{1} \rightarrow a_{2}, \ldots, a_{j-1} \rightarrow a_{j}=a_{1}\right)$ um ciclo negativo em $D(\gamma)$ tal que custo $(C)=-X$, para algum número positivo $X$. Para provar o lema, consideramos algum alinhamento estendido E-ótimo $A=\left[c_{1}, \ldots, c_{n}\right]$ de $(s, t)$ e mostramos que existe um alinhamento estendido $A^{\prime}$ de $(s, t)$ tal que

$$
v \mathrm{E}_{\gamma}\left(A^{\prime}\right)<v \mathrm{E}_{\gamma}(A)
$$

contrariando a otimalidade de $A$.

Como $C$ é um ciclo negativo, $C$ é uma sequência não vazia de arcos, o que implica que $C$ possui pelo menos um arco $a_{1} \rightarrow a_{2}$. Como não existe o arco $\sqcup \rightarrow \sqcup$, ou $a_{1}$ ou $a_{2}$ é diferente de ${ }_{\sqcup}$. Dado que $C$ é um ciclo, considere, sem perda de generalidade, que $a_{1} \neq{ }_{\llcorner}$. Isto implica que $c_{1}(1) \rightarrow a_{1}$ e $a_{1} \rightarrow c_{1}(1)$ são arcos de $D(\gamma)$. Seja um inteiro não negativo $k>\left(\gamma_{c_{1}(1) \rightarrow a_{1}}+\gamma_{a_{1} \rightarrow c_{1}(1)}\right) / X$ e note que o alinhamento $A^{\prime}$ obtido a partir de $A$ substituindo a coluna $c_{1}$ pela coluna

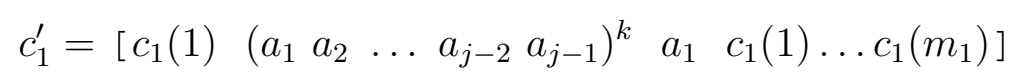

também é um alinhamento de $(s, t)$ e que $v \mathrm{E}_{\gamma}\left(c_{1}^{\prime}\right)=\gamma_{c_{1}(1) \rightarrow \mathrm{a}_{1}}+k(-X)+\gamma_{\mathrm{a}_{1} \rightarrow c_{1}(1)}+v \mathrm{E}_{\gamma}\left(c_{1}\right)$. Sendo, pela definição de $k, \gamma_{c_{1}(1) \rightarrow \mathrm{a}_{1}}+k(-X)+\gamma_{\mathrm{a}_{1} \rightarrow c_{1}(1)}<0$, segue que,

$$
v \mathrm{E}_{\gamma}\left(c_{1}^{\prime}\right)=\gamma_{c_{1}(1) \rightarrow \mathrm{a}_{1}}+k(-X)+\gamma_{\mathrm{a}_{1} \rightarrow c_{1}(1)}+v \mathrm{E}_{\gamma}\left(c_{1}\right)<v \mathrm{E}_{\gamma}\left(c_{1}\right)
$$

Portanto,

$$
v \mathrm{E}_{\gamma}\left(A^{\prime}\right)=v \mathrm{E}_{\gamma}\left[c_{1}^{\prime}, c_{2}, c_{3}, \ldots, c_{n}\right]=v \mathrm{E}_{\gamma}\left(c_{1}^{\prime}\right)+\sum_{i>1} v \mathrm{E}_{\gamma}\left(c_{i}\right)<v \mathrm{E}_{\gamma}\left(c_{1}\right)+\sum_{i>1} v \mathrm{E}_{\gamma}\left(c_{i}\right)=v \mathrm{E}_{\gamma}(A) .
$$

Lema 65. Sejam $s \in \Sigma^{*}$ e $\gamma$ uma matriz de pontuação. Então, opt $\mathrm{E}_{\gamma}(s, s)=0$ se e somente se $D(\gamma)$ não possui ciclo negativo.

Prova. Suponha que opt $\mathrm{E}_{\gamma}(s, s)=0$. Segue da contrapositiva do Lema 64 que $D(\gamma)$ não possui ciclo negativo.

Reciprocamente, suponha que $D(\gamma)$ não possui ciclo negativo. Como

$$
v \mathrm{E}_{\gamma}([s(1), \ldots, s(|s|)])=0
$$

temos que opt $\mathrm{E}_{\gamma}(s, s) \leq 0$. Por outro lado, considere um alinhamento estendido E-ótimo $\left[c_{1}, \ldots, c_{n}\right]$ de $(s, s)$ e, a partir dele, construímos o digrafo $H$, tal que

$$
\begin{aligned}
& V(H)= \Sigma_{\sqcup} \cup \\
&\left\{(1,2),(1,3), \ldots,\left(1, m_{1}-1\right), \ldots,(n, 2),(n, 3), \ldots,\left(n, m_{n}-1\right)\right\}, \\
& E(H)=\left\{(j, i) \rightarrow(j, i+1): \text { para todo } j=1, \ldots, n \text { e } i=2, \ldots, m_{j}-2\right\} \cup \\
&\left\{u \rightarrow(j, 2): \text { para todo } j=1, \ldots, n \text { se } c_{j}(1)=u\right\} \cup \\
&\left\{\left(j, m_{j}-1\right) \rightarrow v: \text { para todo } j=1, \ldots, n \text { se } c_{j}\left(m_{j}\right)=v\right\}, \\
& \text { custo }(u \rightarrow v)= \begin{cases}\gamma_{c_{j}(i) \rightarrow c_{j}(i+1)} & \text { se } u=(j, i) \text { e } v=(j, i+1), \\
\gamma_{c_{j}(1) \rightarrow c_{j}(2)} & \text { se } u=c_{j}(1) \text { e } v=(j, 2), \\
\gamma_{c_{j}\left(m_{j}-1\right) \rightarrow c_{j}\left(m_{j}\right)} & \text { se } u=\left(j, m_{j}-1\right) \text { e } v=c_{j}\left(m_{j}\right) .\end{cases}
\end{aligned}
$$

Note que $H$ é euleriano, custo $(H)=v \mathrm{E}_{\gamma}(A)$ e que cada ciclo $C$ em $H$ é um ciclo em $D(\gamma)$ 
e custo $(C)=v \mathrm{E}_{\gamma}(C)$.

Assim como na construção do digrafo na prova do Lema 41, sendo $H$ euleriano, o Teorema 2 garante que $E(H)$ pode ser decomposto em ciclos arco-disjuntos. Sendo cada um desses ciclos um ciclo em $D(\gamma)$ que, por hipótese não possui ciclo negativo, temos como consequência que

$$
o p t \mathrm{E}_{\gamma}(s, s)=v \mathrm{E}_{\gamma}(A)=\operatorname{custo}(H) \geq 0,
$$

o que nos permite concluir, sendo opt $\mathrm{E}_{\gamma}(s, s) \leq 0$, que opt $\mathrm{E}_{\gamma}(s, s)=0$.

Lema 66. Vale $o p t \mathrm{E}_{\gamma}(s, t) \geq 0$ para cada $s, t \in \Sigma^{*}$ se e somente se $\gamma_{\mathrm{a} \rightarrow \sqcup}, \gamma_{\llcorner\rightarrow \mathrm{a}}, \gamma_{\mathrm{a} \rightarrow \mathrm{b}} \geq 0$, para cada $a, b \in \Sigma$.

Prova. Sejam a, $\mathrm{b} \in \Sigma$ e suponha que $o p t \mathrm{E}_{\gamma}(s, t) \geq 0$ para cada $s, t \in \Sigma^{*}$. Então, $\gamma_{\mathrm{a} \rightarrow \sqcup}=$ $\left.v \mathrm{E}_{\gamma}\left(\left[\mathrm{a}_{\sqcup}\right]\right]\right) \geq \operatorname{opt} \mathrm{E}_{\gamma}(\mathrm{a}, \epsilon) \geq 0$. Analogamente, $\gamma_{\mathrm{L} \rightarrow \mathrm{a}}, \gamma_{\mathrm{a} \rightarrow \mathrm{b}} \geq 0$.

Reciprocamente, sejam $s, t \in \Sigma^{*}$ e suponha que $\gamma_{\mathrm{a} \rightarrow,}, \gamma_{\mathrm{L} \rightarrow \mathrm{a}}, \gamma_{\mathrm{a} \rightarrow \mathrm{b}} \geq 0$ para cada $\mathrm{a}, \mathrm{b} \in \Sigma$ e $A$ é um alinhamento E-ótimo de $(s, t)$. Desde que $v \mathrm{E}_{\gamma}(A)$ é a soma de elementos da entrada de $\gamma$, segue que $v \mathrm{E}_{\gamma}(A) \geq 0$. Concluímos que opt $\mathrm{E}_{\gamma}(s, t)=v \mathrm{E}_{\gamma}(A) \geq 0$.

Lema 67. Vale $\operatorname{opt} \mathrm{E}_{\gamma}(s, t)>0$ para cada $s \neq t \in \Sigma^{*}$ se e somente se

1. $\gamma_{\mathrm{a} \rightarrow \mathrm{a}} \geq 0 \mathrm{e}$

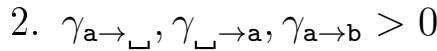

para cada $\mathrm{a} \neq \mathrm{b} \in \Sigma$.

Prova. Sejam $\mathrm{a} \neq \mathrm{b}$ símbolos em $\Sigma$ e suponha que opt $\mathrm{E}_{\gamma}(s, t)>0$ para cada $s \neq t \in \Sigma^{*}$.

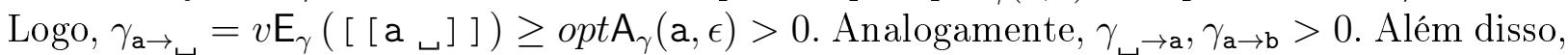
segue do Lema 66 que $\gamma_{\mathrm{a} \rightarrow \mathrm{a}} \geq 0$. Portanto, sendo a, b símbolos arbitrários, se opt $\mathrm{E}_{\gamma}(s, t)>0$ para cada $s \neq t \in \Sigma^{*}$, então $\gamma_{\mathrm{a} \rightarrow \mathrm{a}} \geq 0$ e $\gamma_{\mathrm{a} \rightarrow \mathrm{b}}, \gamma_{\mathrm{b}_{\rightarrow}}, \gamma_{\mathrm{a} \rightarrow \mathrm{b}}>0$ para cada $\mathrm{a} \neq \mathrm{b} \in \Sigma$.

Reciprocamente, sejam $s \neq t$ sequências em $\Sigma^{*}$ e suponha que para cada $\mathrm{a} \neq \mathrm{b} \in \Sigma$, $\gamma_{\mathrm{a} \rightarrow \mathrm{a}} \geq 0$ e $\gamma_{\mathrm{a} \rightarrow \mathrm{b}}, \gamma_{\mathrm{s}_{\rightarrow \mathrm{a}}}, \gamma_{\mathrm{a} \rightarrow \mathrm{b}}>0$. Seja $\left[c_{1}, \ldots, c_{n}\right]$ um alinhamento E-ótimo de $(s, t)$. Como $v \mathrm{E}_{\gamma}\left(c_{i}\right)$ é a soma de entradas em $\gamma$ que não possui entradas negativas, temos que $v \mathrm{E}_{\gamma}\left(c_{i}\right) \geq 0$ para cada $i$. Sendo $s \neq t$, existe pelo menos uma coluna $j$ tal que $c_{j}(1) \neq c_{j}\left(m_{j}\right)$, o que implica que existe $k$ tal que $c_{j}(k) \neq c_{j}(k+1)$. Segue que

$$
v \mathrm{E}_{\gamma}\left(c_{j}\right)=\gamma_{c_{j}(k) \rightarrow c_{j}(k+1)}+\sum_{l=1, l \neq k}^{m_{l}-1} \gamma_{c_{j}(l) \rightarrow c_{j}(l+1)} \geq \gamma_{c_{j}(k) \rightarrow c_{j}(k+1)}+0>0 .
$$

Portanto,

$$
o p t \mathrm{E}_{\gamma}(s, t)=v \mathrm{E}_{\gamma}\left(\left[c_{1}, \ldots, c_{n}\right]\right)=v \mathrm{E}_{\gamma}\left(c_{j}\right)+\sum_{i \neq j} v \mathrm{E}_{\gamma}\left(c_{i}\right) \geq v \mathrm{E}_{\gamma}\left(c_{j}\right)+0>0 .
$$

Logo, sendo $s \neq t$ arbitrárias, segue que se $\gamma_{\mathrm{a} \rightarrow \mathrm{a}} \geq 0$ e $\gamma_{\mathrm{a} \rightarrow}, \gamma_{\mathbf{b}} \rightarrow \mathrm{a}, \gamma_{\mathrm{a} \rightarrow \mathrm{b}}>0$ para cada $\mathrm{a} \neq \mathrm{b} \in \Sigma$, então opt $\mathrm{E}_{\gamma}(s, t)>0$ para cada $s \neq t \in \Sigma^{*}$.

Sejam $\mathrm{a}, \mathrm{b} \in \gamma$. Denotamos o custo de um caminho ótimo de $\mathrm{a} a \mathrm{~b}$ em $\gamma$ por $\mathcal{P}_{\gamma}(\mathrm{a}, \mathrm{b})$.

Lema 68. Seja $\gamma$ uma matriz de pontuação. Então, opt $\mathrm{E}_{\gamma}(s, t)=o p t \mathrm{E}_{\gamma}(t, s)$ para cada $s, t \in \Sigma^{*}$ se e somente se $\mathcal{P}_{\gamma}\left(\mathrm{a},{ }_{\iota}\right)=\mathcal{P}_{\gamma}(\sqcup, \mathrm{a})$ e $\mathcal{P}_{\gamma}(\mathrm{a}, \mathrm{b})=\mathcal{P}_{\gamma}(\mathrm{b}, \mathrm{a})$ para cada $\mathrm{a} \neq \mathrm{b} \in \Sigma$. 
Prova. Sejam $\mathrm{a} \neq \mathrm{b} \in \Sigma$ e suponha que $\operatorname{opt} \mathrm{E}_{\gamma}(s, t)=o p t \mathrm{E}_{\gamma}(t, s)$ para cada $s, t \in \Sigma^{*}$. Qualquer alinhamento estendido de $(\mathrm{a}, \epsilon)$ possui uma única coluna. Logo, o caminho $P$ de a para $\sqcup$ em $D(\gamma)$ que é correspondente à única coluna de um alinhamento E-ótimo de $(\mathrm{a}, \epsilon)$ tem custo opt $\mathrm{E}_{\gamma}(\mathrm{a}, \epsilon)$ o que implica $\mathcal{P}_{\gamma}\left(\mathrm{a},{ }_{\lrcorner}\right) \leq \operatorname{custo}(P)=o p t \mathrm{E}_{\gamma}(\mathrm{a}, \epsilon)$. Similarmente, um alinhamento estendido $A$ de $(\mathrm{a}, \epsilon)$ que corresponde a um caminho ótimo de a para $\sqcup$ tem custo $\left.\mathcal{P}_{\gamma}\left(\mathrm{a}_{{ }}\right)\right)$o que implica que opt $\mathrm{E}_{\gamma}(\mathrm{a}, \epsilon) \leq v \mathrm{E}_{\gamma}(A)=\mathcal{P}_{\gamma}\left(\mathrm{a},{ }_{\iota}\right)$. Segue que opt $\mathrm{E}_{\gamma}(\mathrm{a}, \epsilon)=\mathcal{P}_{\gamma}(\mathrm{a}$, s $)$. Usando argumentação similar, temos opt $\mathrm{E}_{\gamma}(\epsilon, \mathrm{a})=\mathcal{P}_{\gamma}(\sqcup, \mathrm{a})$. Segue, sendo opt $\mathrm{E}_{\gamma}(\mathrm{a}, \epsilon)=\operatorname{opt} \mathrm{E}_{\gamma}(\epsilon, \mathrm{a})$, que $\mathcal{P}_{\gamma}(\mathrm{a}, \iota)=\operatorname{opt} \mathrm{E}_{\gamma}(\mathrm{a}, \epsilon)=\operatorname{opt} \mathrm{E}_{\gamma}(\epsilon, \mathrm{a})=\mathcal{P}_{\gamma}(\iota, \mathrm{a})$.

Um alinhamento E-ótimo de $(\mathrm{a}, \mathrm{b})$ possui uma ou duas colunas. Se ele tem somente uma coluna, com uma argumentação similar à feita no parágrafo anterior, segue que $\mathcal{P}_{\gamma}(\mathrm{a}, \mathrm{b})=$ $\mathcal{P}_{\gamma}(\mathrm{b}, \mathrm{a})$. Se o alinhamento E-ótimo de $(\mathrm{a}, \mathrm{b})$ possui duas colunas, digamos

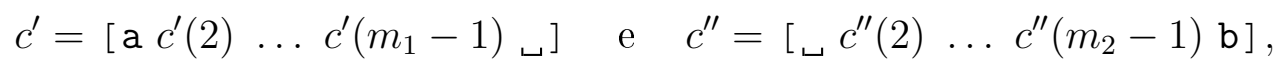

então definimos o alinhamento de $(\mathrm{a}, \mathrm{b})$ com uma única coluna

$$
c=\left[\begin{array}{lllll}
\mathrm{a} & c^{\prime}(2) & \ldots & c^{\prime}\left(m_{j}-1\right) \_ & c^{\prime \prime}(2) \ldots c^{\prime \prime}\left(m_{2}-1\right) \mathrm{b}
\end{array}\right]
$$

onde verificamos que $v \mathrm{E}_{\gamma}\left(\left[c_{1}, c_{2}\right]\right)=v \mathrm{E}_{\gamma}([c])$, o que implica que o alinhamento estendido [c] também é um alinhamento E-ótimo de (a,b). Logo, novamente usando uma argumentação similar ao parágrafo anterior, segue que $\mathcal{P}_{\gamma}(\mathrm{a}, \mathrm{b})=\mathcal{P}_{\gamma}(\mathrm{b}, \mathrm{a})$. Portanto, pelos argumentos dos dois primeiros parágrafos, se opt $\mathrm{E}_{\gamma}(s, t)=o p t \mathrm{E}_{\gamma}(t, s)$ para cada $s, t \in \Sigma^{*}$, então $\mathcal{P}_{\gamma}(\mathrm{a}, \sqcup)=\mathcal{P}_{\gamma}(\sqcup, \mathrm{a})$ e $\mathcal{P}_{\gamma}(\mathrm{a}, \mathrm{b})=\mathcal{P}_{\gamma}(\mathrm{b}, \mathrm{a})$ para cada $\mathrm{a} \neq \mathrm{b} \in \Sigma$.

Reciprocamente, sejam $s, t \in \Sigma^{*}$ e suponha que $\mathcal{P}_{\gamma}\left(\mathrm{a},{ }_{\iota}\right)=\mathcal{P}_{\gamma}\left({ }_{\iota}, \mathrm{a}\right)$ e $\mathcal{P}_{\gamma}(\mathrm{a}, \mathrm{b})=\mathcal{P}_{\gamma}(\mathrm{b}, \mathrm{a})$ para cada $\mathrm{a} \neq \mathrm{b} \in \Sigma$ e que $A=\left[c_{1}, \ldots, c_{n}\right]$ é um alinhamento estendido E-ótimo de $(s, t)$. Cada $c_{i}$ corresponde, pela otimalidade de $A$, a um caminho de custo mínimo de $c_{i}(1)$ a $c_{i}\left(m_{i}\right)$ em $D(\gamma)$ o que implica que

$$
o p t \mathrm{E}_{\gamma}(s, t)=\sum_{i} v \mathrm{E}_{\gamma}\left(c_{i}\right)=\sum_{i} \mathcal{P}_{\gamma}\left(c_{i}(1), c_{i}\left(m_{i}\right)\right)
$$

Considere um alinhamento estendido $B=\left[c_{1}^{\prime}, \ldots, c_{n}^{\prime}\right]$ onde cada coluna $c_{i}^{\prime}$ é definida por um caminho de custo mínimo de $c_{i}\left(m_{i}\right)$ a $c_{i}(1)$. Claramente $B$ é um alinhamento estendido de $(t, s)$ com pontuação igual a $\sum_{i} \mathcal{P}_{\gamma}\left(c_{i}\left(m_{i}\right), c_{i}(1)\right)$. Desde que $\mathcal{P}_{\gamma}\left(c_{i}(1), c_{i}\left(m_{i}\right)\right)=$ $\mathcal{P}_{\gamma}\left(c_{i}\left(m_{i}\right), c_{i}(1)\right)$ para cada $i$, segue que

$$
\begin{aligned}
o p t \mathrm{E}_{\gamma}(s, t) & =\sum_{i} v \mathrm{E}_{\gamma}\left(c_{i}\right)=\sum_{i} \mathcal{P}_{\gamma}\left(c_{i}(1), c_{i}\left(m_{i}\right)\right) \\
& =\sum_{i} \mathcal{P}_{\gamma}\left(c_{i}\left(m_{i}\right), c_{i}(1)\right)=v \mathrm{E}_{\gamma}\left(\left[c_{1}^{\prime}, \ldots, c_{n}^{\prime}\right]\right) \\
& \leq o p t \mathrm{E}_{\gamma}(t, s) .
\end{aligned}
$$

Usando raciocínio análogo, segue que opt $\mathrm{E}_{\gamma}(t, s) \leq o p t \mathrm{E}_{\gamma}(s, t)$ o que nos permite concluir que opt $\mathrm{E}_{\gamma}(s, t)=o p t \mathrm{E}_{\gamma}(t, s)$.

Lema 69. Se $D(\gamma)$ não possui ciclo de custo negativo, então

$$
o p t \mathrm{E}_{\gamma}(s, u) \leq o p t \mathrm{E}_{\gamma}(s, t)+o p t \mathrm{E}_{\gamma}(t, u)
$$

para todo $s, t, u \in \Sigma^{*}$. 
Prova. Sejam $s, t, u \in \Sigma^{*},\left[c_{1}, \ldots, c_{n_{c}}\right]$ e $\left[e_{1}, \ldots, e_{n_{e}}\right]$ alinhamentos E-ótimos de $(s, t)$ e $(t, u)$, respectivamente, e os inteiros $1 \leq i_{1}<\ldots<i_{|t|} \leq n_{c}$ e $1 \leq j_{1}<j_{2}<\ldots<j_{|t|} \leq n_{e}$ tais que $t(k)=c_{i_{k}}\left(m_{i_{k}}\right)=e_{j_{k}}(1)$ e suponha que $D(\gamma)$ não possui ciclo de custo negativo.

Para cada $k=1, \ldots,|t|$, temos

$$
v \mathrm{E}_{\gamma}\left(c_{i_{k}}\right)+v \mathrm{E}_{\gamma}\left(e_{j_{k}}\right)=v \mathrm{E}_{\gamma}\left(\left[c_{i_{k}}(1) \ldots c_{i_{k}}\left(m_{i_{k}}-1\right) t(k) e_{j_{k}}(2) \ldots e_{j_{k}}\left(m_{j_{k}}\right)\right]\right) .
$$

$\operatorname{Sejam} \mathcal{C}_{1}=\left\{k \in\left\{1, \ldots, n_{c}\right\}: k \neq i_{1}, \ldots, i_{|t|}\right\}, \mathcal{C}_{2}=\left\{i_{k}: c_{i_{k}}(1) \neq{ }\right.$ ou $\left.e_{j_{k}}\left(m_{j_{k}}\right) \neq{ }_{\sqcup}\right\}$, $\mathcal{C}_{3}=\left\{i_{k}: c_{i_{k}}(1)={ }_{\iota}\right.$ e $\left.e_{j_{k}}\left(m_{j_{k}}\right)={ }_{\iota}\right\}, \mathcal{E}_{1}=\left\{k \in\left\{1, \ldots, n_{e}\right\}: k \neq j_{1}, \ldots, j_{|t|}\right\}, \mathcal{E}_{2}=\left\{j_{k}:\right.$ $c_{i_{k}}(1) \neq{ }$ ou $\left.e_{j_{k}}\left(m_{j_{k}}\right) \neq{ }\right\}$ e $\mathcal{E}_{3}=\left\{j_{k}: c_{i_{k}}(1)={ }_{\iota}\right.$ e $\left.e_{j_{k}}\left(m_{j_{k}}\right)={ }\right\}$. Note que

$$
\begin{aligned}
v \mathrm{E}_{\gamma}\left(\left[c_{1}, \ldots, c_{n_{c}}\right]\right) & =\sum_{k \in \mathcal{C}_{1}} v \mathrm{E}_{\gamma}\left(c_{k}\right)+\sum_{i_{k} \in \mathcal{C}_{2}} v \mathrm{E}_{\gamma}\left(c_{i_{k}}\right)+\sum_{i_{k} \in \mathcal{C}_{3}} v \mathrm{E}_{\gamma}\left(c_{i_{k}}\right), \\
v \mathrm{E}_{\gamma}\left(\left[e_{1}, \ldots, e_{n_{e}}\right]\right) & =\sum_{k \in \mathcal{E}_{1}} v \mathrm{E}_{\gamma}\left(e_{k}\right)+\sum_{j_{k} \in \mathcal{E}_{2}} v \mathrm{E}_{\gamma}\left(e_{j_{k}}\right)+\sum_{j_{k} \in \mathcal{E}_{3}} v \mathrm{E}_{\gamma}\left(e_{j_{k}}\right) .
\end{aligned}
$$

Como consequência de (4.28), temos que

$$
\begin{aligned}
\sum_{i_{k} \in \mathcal{C}_{2}} v \mathrm{E}_{\gamma}\left(c_{i_{k}}\right)+\sum_{j_{k} \in \mathcal{E}_{2}} v \mathrm{E}_{\gamma}\left(e_{j_{k}}\right) & =\sum_{i_{k} \in \mathcal{C}_{2}}\left(v \mathrm{E}_{\gamma}\left(\left[c_{i_{k}}(1) \ldots t(k) \ldots e_{j_{k}}\left(m_{j_{k}}\right)\right]\right)\right. \\
\sum_{i_{k} \in \mathcal{C}_{3}} v \mathrm{E}_{\gamma}\left(c_{i_{k}}\right)+\sum_{j_{k} \in \mathcal{E}_{3}} v \mathrm{E}_{\gamma}\left(e_{j_{k}}\right) & =\sum_{i_{k} \in \mathcal{C}_{3}}\left(v \mathrm{E}_{\gamma}\left(\left[c_{\sqcup} c_{i_{k}}(2) \ldots t(k) e_{j_{k}}(2) \ldots \sqcup\right]\right)\right) \geq 0
\end{aligned}
$$

onde a desigualdade em (4.32) segue de $D(\gamma)$ não possuir ciclo de custo negativo.

Seja $A$ um alinhamento estendido de $(s, u)$ cujas colunas são definidas de acordo com as seguintes regras: para cada $k \in \mathcal{C}_{1}$, a coluna $c_{k}$ é coluna de $A$; para cada $k \in \mathcal{E}_{1}$, a coluna $e_{k}$ é coluna de $A$; e para cada $k=1, \ldots,|t|$ tal que $i_{k} \in \mathcal{C}_{2}$ a coluna

$$
\left[c_{i_{k}}(1) c_{i_{k}}(2) \ldots c_{i_{k}}\left(m_{i_{k}}-1\right) t(k) e_{j_{k}}(2) e_{j_{k}}(3) \ldots e_{j_{k}}\left(m_{j_{k}}\right)\right]
$$

é coluna de $A$. Logo,

$$
\begin{aligned}
v \mathrm{E}_{\gamma}(A)= & \sum_{k \in \mathcal{C}_{1}} v \mathrm{E}_{\gamma}\left(c_{k}\right)+\sum_{k \in \mathcal{E}_{1}} v \mathrm{E}_{\gamma}\left(e_{k}\right)+\sum_{i_{k} \in \mathcal{C}_{2}}\left(v \mathrm{E}_{\gamma}\left(\left[c_{i_{k}}(1) \ldots t(k) \ldots e_{j_{k}}\left(m_{j_{k}}\right)\right]\right)\right. \\
= & \sum_{k \in \mathcal{C}_{1}} v \mathrm{E}_{\gamma}\left(c_{k}\right)+\sum_{k \in \mathcal{E}_{1}} v \mathrm{E}_{\gamma}\left(e_{k}\right)+\sum_{i_{k} \in \mathcal{C}_{2}} v \mathrm{E}_{\gamma}\left(c_{i_{k}}\right)+\sum_{j_{k} \in \mathcal{E}_{2}} v \mathrm{E}_{\gamma}\left(e_{j_{k}}\right) \\
\leq & \sum_{k \in \mathcal{C}_{1}} v \mathrm{E}_{\gamma}\left(c_{k}\right)+\sum_{k \in \mathcal{E}_{1}} v \mathrm{E}_{\gamma}\left(e_{k}\right)+\sum_{i_{k} \in \mathcal{C}_{2}} v \mathrm{E}_{\gamma}\left(c_{i_{k}}\right)+\sum_{j_{k} \in \mathcal{E}_{2}} v \mathrm{E}_{\gamma}\left(e_{j_{k}}\right)+ \\
& \sum_{i_{k} \in \mathcal{C}_{3}} v \mathrm{E}_{\gamma}\left(c_{i_{k}}\right)+\sum_{j_{k} \in \mathcal{E}_{3}} v \mathrm{E}_{\gamma}\left(e_{j_{k}}\right) \\
= & v \mathrm{E}_{\gamma}\left(\left[c_{1}, \ldots, c_{n_{c}}\right]+v \mathrm{E}_{\gamma}\left(\left[e_{1}, \ldots, e_{n_{e}}\right]\right)\right. \\
= & o p t \mathrm{E}_{\gamma}(s, t)+o p t \mathrm{E}_{\gamma}(t, u)
\end{aligned}
$$

onde (4.33) segue de (4.31); (4.34) segue de (4.32); (4.35) segue de (4.29) e (4.30); e (4.36) segue de $\left[c_{1}, \ldots, c_{n_{c}}\right]$ e $\left[e_{1}, \ldots, e_{n_{e}}\right]$ serem alinhamentos E-ótimos de $(s, t)$ e de $(t, u)$. Desde que opt $\mathrm{E}_{\gamma}(s, u) \leq v \mathrm{E}_{\gamma}(A)$, segue que

$$
o p t \mathrm{E}_{\gamma}(s, u) \leq v \mathrm{E}_{\gamma}(A) \leq o p t \mathrm{E}_{\gamma}(s, t)+o p t \mathrm{E}_{\gamma}(t, u) .
$$


Portanto, desde que $s, t, u$ são sequências arbitrárias, segue que se $D(\gamma)$ não possui ciclo de custo negativo, então opt $\mathrm{E}_{\gamma}(s, u) \leq o p t \mathrm{E}_{\gamma}(s, t)+o p t \mathrm{E}_{\gamma}(t, u)$ para todo $s, t, u \in \Sigma^{*}$.

Teorema 70. Vale opt $\mathrm{E}_{\gamma} \in \mathcal{M}$ se e somente se vale $\gamma \in \mathbb{M}^{\mathrm{E}}$.

Prova. Suponha que opt $\mathrm{E}_{\gamma} \in \mathcal{M}$. Logo,

(i) $\operatorname{opt} \mathrm{E}_{\gamma}(s, t)>0 \mathrm{e}$

(ii) opt $\mathrm{E}_{\gamma}(s, t)=o p t \mathrm{E}_{\gamma}(t, s)$

para cada $s \neq t \in \Sigma^{*}$. Como vale $(i)$, temos pelo Lema 67 que $\gamma_{\mathrm{a} \rightarrow \mathrm{b}}, \gamma_{\mathrm{a} \rightarrow\lrcorner}, \gamma_{\mathrm{c} \rightarrow \mathrm{a}}>0$ e $\gamma_{\mathrm{a} \rightarrow \mathrm{a}} \geq 0$, e como vale $(\mathrm{ii})$, temos pelo Lema 68 que $\mathcal{P}_{\gamma}\left(\mathrm{a},{ }_{\lrcorner}\right)=\mathcal{P}_{\gamma}\left({ }_{\iota}, \mathrm{a}\right)$ e $\mathcal{P}_{\gamma}(\mathrm{a}, \mathrm{b})=\mathcal{P}_{\gamma}(\mathrm{b}, \mathrm{a})$ para cada $\mathrm{a} \neq \mathrm{b} \in \Sigma$. Segue daí que $\gamma \in \mathbb{M}^{\mathrm{E}}$.

Reciprocamente, suponha que $\gamma \in \mathbb{M}^{\mathrm{E}}$. Temos por definição de $\mathbb{M}^{\mathrm{E}}$ que

(a) $\gamma_{\mathrm{a} \rightarrow \mathrm{a}} \geq 0, \gamma_{\mathrm{a} \rightarrow \mathrm{b}}, \gamma_{\mathrm{a} \rightarrow \boldsymbol{\omega}}, \gamma_{\boldsymbol{\omega} \rightarrow \mathrm{a}}>0$,

(b) $\left.\mathcal{P}_{\gamma}\left(\mathrm{a},{ }_{\iota}\right)=\mathcal{P}_{\gamma}(\lrcorner, \mathrm{a}\right)$ e $\mathcal{P}_{\gamma}(\mathrm{a}, \mathrm{b})=\mathcal{P}_{\gamma}(\mathrm{b}, \mathrm{a})$

para todo $\mathrm{a} \neq \mathrm{b} \in \Sigma$. Como vale $(a)$, temos que $D(\gamma)$ não possui ciclo negativo, o que implica pelo Lema 65 que opt $\mathrm{E}_{\gamma}(s, s)=0$ e pelo Lema 69 que opt $\mathrm{E}_{\gamma}(s, u) \leq o p t \mathrm{E}_{\gamma}(s, t)+o p t \mathrm{E}_{\gamma}(t, u)$ para todo $s, t, u \in \Sigma^{*}$. Temos também pelo Lema 67 que opt $\mathrm{E}_{\gamma}(s, t)>0$ para todo $s \neq t \in \Sigma^{*}$. Como vale $(b)$, temos pelo Lema 68 que opt $\mathrm{E}_{\gamma}(s, t)=$ opt $\mathrm{E}_{\gamma}(t, s)$ para todo $s, t \in \Sigma^{*}$. Segue daí que opt $\mathrm{E}_{\gamma} \in \mathcal{M}$.

\subsection{Comentário final}

Neste capítulo determinamos condições suficientes e necessárias para que a matriz de pontuação $\gamma$ induza cada uma das propriedades de uma métrica nas funções opt $\mathrm{A}_{\gamma}$, opt $\mathrm{N}_{\gamma}$ e opt $\mathrm{E}_{\gamma}$. Podemos observar que, em qualquer um dos casos, cada propriedade pode ser verificada gastando tempo $O\left(|\Sigma|^{3}\right)$. 


\section{Capítulo 5}

\section{Matrizes equivalentes}

A função $v \mathrm{~A}_{\gamma}$ é um critério que, como vimos, pode ser utilizada para "classificar" alinhamentos. Informalmente falando, bons alinhamentos possuem pontuação baixa e alinhamentos ruins possuem pontuação alta. Note que uma classificação obtida usando esse critério depende também dos valores em $\gamma$. Por exemplo, considere as seguintes matrizes de pontuação:

$$
\gamma=\begin{array}{c|ccc} 
& \mathrm{a} & \mathrm{b} & \mathrm{u} \\
\hline \mathrm{a} & 0 & 1 & 1 \\
\mathrm{~b} & 1 & 0 & 1 \\
- & 1 & 1
\end{array} \quad \text { e } \delta=\begin{array}{r|ccc}
\mathrm{a} & 0 & 1 & 2 \\
\mathrm{~b} & 1 & 0 & 2 \\
& 2 & 2
\end{array}
$$

e os seguintes alinhamentos de (aba, bab):

$$
A=\left[\begin{array}{lll}
\mathrm{a} & \mathrm{b} & \mathrm{a} \\
\mathrm{b} & \mathrm{a} & \mathrm{b}
\end{array}\right] \text { e } B=\left[\begin{array}{cccc}
\mathrm{a} & \mathrm{b} & \mathrm{a} & \sqcup \\
& \mathrm{b} & \mathrm{a} & \mathrm{b}
\end{array}\right]
$$

Se $\gamma$ é a matriz de pontuação, então $B$ é considerado um alinhamento melhor do que $A$

$$
v \mathrm{~A}_{\gamma}(A)=\gamma_{\mathrm{a} \rightarrow \mathrm{b}}+\gamma_{\mathrm{b} \rightarrow \mathrm{a}}+\gamma_{\mathrm{a} \rightarrow \mathrm{b}}=3>2=\gamma_{\mathrm{a} \rightarrow \sqcup}+\gamma_{\mathrm{b} \rightarrow \mathrm{b}}+\gamma_{\mathrm{a} \rightarrow \mathrm{a}}+\gamma_{\llcorner\rightarrow \mathrm{b}}=v \mathrm{~A}_{\gamma}(B) .
$$

e se $\delta$ é a matriz de pontuação, então é $A$ que é considerado um alinhamento melhor pois, neste caso, $v \mathrm{~A}_{\delta}(A)=3<4=v \mathrm{~A}_{\delta}(B)$.

Entretanto, não é para qualquer par de matrizes de pontuação $\gamma \neq \delta$ que encontramos um par de alinhamentos $A$ e $B$ de duas sequências onde $v \mathrm{~A}_{\gamma}(A)<v \mathrm{~A}_{\gamma}(B)$ e $v \mathrm{~A}_{\delta}(A) \geq v \mathrm{~A}_{\delta}(B)$. Para $\gamma$ e $\delta$ matrizes de pontuação, escrevemos $\gamma \sim \delta$ e dizemos que $\gamma$ e $\delta$ são equivalentes se para cada par de alinhamentos $A$ e $B$ de quaisquer duas sequências, vale

$$
v \mathrm{~A}_{\gamma}(A) \leq v \mathrm{~A}_{\gamma}(B) \text { se e somente se } v \mathrm{~A}_{\delta}(A) \leq v \mathrm{~A}_{\delta}(B)
$$

Da definição são imediatas as seguintes propriedades para $\gamma, \delta, \varphi$ matrizes de pontuação: $\gamma \sim \gamma$ (reflexibilidade), $\gamma \sim \delta$ implica que $\delta \sim \gamma$ (simetria) e $\gamma \sim \delta$ e $\delta \sim \varphi$ implica que $\gamma \sim \varphi$ (transitividade), o que significa que $\sim$ é uma relação de equivalência no conjunto das matrizes de pontuação.

O Problema APS é formulado neste texto como um problema de minimização que é o de encontrar um alinhamento $A$ tal que $v \mathrm{~A}_{\gamma}(A)$ seja o menor possível. Com matrizes de pontuação apropriadas, esse mesmo problema pode ser formulado como um problema de maximização: encontrar um alinhamento $A$ tal que $v \mathrm{~A}_{\gamma}(A)$ seja o maior possível. Setubal e Meidanis [SM97] mostram como determinar, a partir de uma matriz de pontuação $\gamma$ que é utilizada para o Problema APS, uma matriz de pontuação $\delta$ que é utilizada para um 
problema de maximização de modo que $v \mathrm{~A}_{\gamma}(A) \leq v \mathrm{~A}_{\gamma}(B)$ se e somente se $v \mathrm{~A}_{\delta}(A) \geq v \mathrm{~A}_{\delta}(B)$ para cada par de alinhamentos $A$ e $B$ de quaisquer pares de sequências. Neste capítulo, adaptamos o estudo de Meidanis e Setubal para caracterizar pares de matrizes equivalentes, ou mais precisamente, determinamos quais são as condições necessárias e suficientes para que duas matrizes sejam equivalentes. Duas operações em matrizes de pontuação são definidas. Em uma dessas operações, a operação de multiplicação, cada elemento da matriz é multiplicado por um número positivo e, em outra, a operação de soma, um número é adicionado a cada elemento da matriz de pontuação. Mostramos, para um amplo conjunto de matrizes de pontuação, que se duas matrizes pertencem a uma mesma classe de equivalência, então uma pode ser obtida a partir da outra usando essas operações de multiplicação e de soma.

Denotamos $\gamma=0$ se cada entrada da matriz de pontuação $\gamma$ é zero, e $\gamma \neq 0$ caso contrário.

\subsection{Operações $\otimes \mathrm{e} \oplus$ nas matrizes de pontuação}

Sejam $x \in \mathbb{R}$ e $\gamma$ uma matriz de pontuação. A expressão $x \otimes \gamma$, que dizemos ser a multiplicação do número $x$ pela matriz $\gamma$, denota uma matriz de pontuação $\delta$ tal que

$$
\delta_{\mathrm{a} \rightarrow \mathrm{b}}=x \cdot \gamma_{\mathrm{a} \rightarrow \mathrm{b}}, \quad \delta_{\mathrm{a} \rightarrow \sqcup}=x \cdot \gamma_{\mathrm{a} \rightarrow\lrcorner}, \quad \delta_{\lrcorner \rightarrow \mathrm{b}}=x \cdot \gamma_{\lrcorner \rightarrow \mathrm{b}}
$$

e a expressão $x \oplus \gamma$, que dizemos ser a soma do número y pela matriz $\gamma$, denota uma matriz de pontuação $\varphi$ tal que

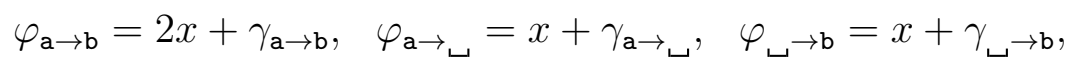

para cada $\mathrm{a}, \mathrm{b} \in \Sigma$. Por exemplo, se

$$
\gamma=\begin{array}{c|ccc} 
& \mathrm{a} & \mathrm{b} & \mathrm{c} \\
\hline \mathrm{a} & 0 & 2 & 2 \\
\mathrm{~b} & 1 & 0 & 1 \\
- & 3 & 1 &
\end{array}
$$

então a matriz de pontuação $\delta=2 \oplus(4 \otimes \gamma)$ é

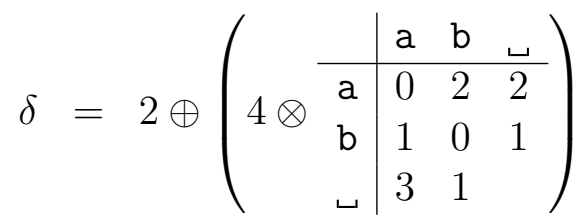

$$
\begin{aligned}
& =2 \oplus \begin{array}{c|ccc} 
& \mathrm{a} & \mathrm{b} & \mathrm{u} \\
\hline \mathrm{a} & 0 & 8 & 8 \\
\mathrm{~b} & 4 & 0 & 4
\end{array} \\
& \begin{array}{c|cc} 
& 12 & 4
\end{array} \\
& =\begin{array}{c|ccc} 
& \mathrm{a} & \mathrm{b} & \mathrm{c} \\
\hline \mathrm{a} & 4 & 12 & 10 \\
\mathrm{~b} & 8 & 4 & 6 \\
\mathrm{c} & 14 & 6
\end{array} .
\end{aligned}
$$

Note que em uma expressão composta por operadores $\otimes \mathrm{e} \oplus$, a operação mais a direita na expressão deve ser executada primeiro. Dado que a matriz de pontuação é o operando que fica mais a direita, essa é a única precedência que faz sentido. Entretanto, os parênteses 
são colocados para enfatizar essa ordem.

Para $\gamma, \delta$ e $\varphi$ matrizes de pontuação e $x, y$ e $z$ números com $x \neq 0$, podemos verificar cada uma das seguintes propriedades:

P1. $1 \otimes \gamma=\gamma$,

P2. $0 \oplus \gamma=\gamma$,

P3. Se $\delta=x \otimes \gamma$, então $\gamma=(1 / x) \otimes \delta$,

P4. Se $\delta=y \oplus \gamma$, então $\gamma=-y \oplus \delta$,

P5. $y \oplus(x \otimes \gamma)=x \otimes((y / x) \oplus \gamma)$,

P6. $z \oplus(y \oplus \gamma)=(z+y) \oplus \gamma$,

P7. $z \otimes(y \otimes \gamma)=(z \cdot y) \otimes \gamma$.

Além disso, note que, para qualquer alinhamento $A$ de um par de sequências $(s, t)$, temos

$$
\begin{aligned}
& v \mathrm{~A}_{x \otimes \gamma}(A)=x \cdot v \mathrm{~A}_{\gamma}(A), \\
& v \mathrm{~A}_{y \oplus \gamma}(A)=y(|s|+|t|)+v \mathrm{~A}_{\gamma}(A) .
\end{aligned}
$$

Sejam $\gamma$ e $\delta$ matrizes de pontuação. Dizemos que $\delta$ é gerada por $\gamma$ em $n$ passos se $n=0$ e $\delta=\gamma$, ou então $n>0$ e existe uma matriz de pontuação $\varphi$ que é gerada por $\gamma$ em $n-1$ passos e $\delta=x$ op $\varphi$, onde op $=\otimes$ ou op $=\oplus$ e $x$ é um número que é positivo se op $=\otimes$. O próximo resultado mostra que se uma matriz pode ser gerada a partir de outra, isto pode ser feito por uma simples operação de soma seguida por uma de multiplicação.

Fato 71. Sejam $\gamma$ e $\delta$ matrizes de pontuação. Para qualquer número natural $n$, se $\delta$ é gerada por $\gamma$ em $n$ passos, então existem $x>0$ e $y$ números tais que $\delta=x \otimes(y \oplus \gamma)$.

Prova. A prova é feita por indução em $n$. Se $n=0$, então $\delta=\gamma$ e vale $\delta=1 \otimes(0 \oplus \gamma)$. Suponha que $n>0$ e, portanto, existe uma matriz de pontuação $\varphi$ que é gerada por $\gamma$ em $n-1$ passos e $\delta=x$ op $\varphi$, onde op $=\otimes$ ou op $=\oplus$ e $x$ é um número que é positivo se op $=\otimes$. Por hipótese de indução, existem $x^{\prime}>0$ e $y^{\prime}$ números tais que $\varphi=x^{\prime} \otimes\left(y^{\prime} \oplus \gamma\right)$. Logo, $\delta=x$ op $\left(x^{\prime} \otimes\left(y^{\prime} \oplus \gamma\right)\right)$. Se op $=\otimes$, então, usando $\mathbf{P} 7$, temos que

$$
\delta=x \text { op }\left(x^{\prime} \otimes\left(y^{\prime} \oplus \gamma\right)\right)=x \otimes\left(x^{\prime} \otimes\left(y^{\prime} \oplus \gamma\right)\right)=\left(x \cdot x^{\prime}\right) \otimes\left(y^{\prime} \oplus \gamma\right),
$$

onde, desde que $x>0$ e $x^{\prime}>0$, temos que $x \cdot x^{\prime}>0$ e o fato está provado. Se op $=\oplus$, então, usando $\mathbf{P 5}$ e $\mathbf{P 6}$, temos que

$$
\begin{aligned}
\delta & =x \text { op }\left(x^{\prime} \otimes\left(y^{\prime} \oplus \gamma\right)\right)=x \oplus\left(x^{\prime} \otimes\left(y^{\prime} \oplus \gamma\right)\right)=x^{\prime} \otimes\left(\frac{x}{x^{\prime}} \oplus\left(y^{\prime} \oplus \gamma\right)\right) \\
& =x^{\prime} \otimes\left(\left(\frac{x}{x^{\prime}}+y^{\prime}\right) \oplus \gamma\right)
\end{aligned}
$$

e também neste caso o fato está provado.

O lema a seguir mostra que uma relação entre matrizes geradas e matrizes equivalentes.

Lema 72. Sejam $\gamma$ uma matriz de pontuação, $x$ um número positivo e $y$ um número. Então, $\gamma \sim x \otimes(y \oplus \gamma)$. 
Prova. Sejam $A$ e $B$ alinhamentos de duas sequências de comprimentos $n$ e $m$. Para demonstrar o lema, mostramos que $v \mathrm{~A}_{\gamma}(A) \leq v \mathrm{~A}_{\gamma}(B)$ implica em $v \mathrm{~A}_{x \otimes(y \oplus \gamma)}(A) \leq v \mathrm{~A}_{x \otimes(y \oplus \gamma)}(B)$ e, depois, que $v \mathrm{~A}_{x \otimes(y \oplus \gamma)}(A) \leq v \mathrm{~A}_{x \otimes(y \oplus \gamma)}(B)$ implica em $v \mathrm{~A}_{\gamma}(A) \leq v \mathrm{~A}_{\gamma}(B)$.

Suponha que $v \mathrm{~A}_{\gamma}(A) \leq v \mathrm{~A}_{\gamma}(B)$. Logo,

$$
\begin{aligned}
v \mathrm{~A}_{x \otimes(y \oplus \gamma)}(A) & =x \cdot v \mathrm{~A}_{y \oplus \gamma}(A)=x\left(y(n+m)+v \mathrm{~A}_{\gamma}(A)\right) \\
& \leq x\left(y(n+m)+v \mathrm{~A}_{\gamma}(B)\right) \\
& =v \mathrm{~A}_{x \otimes(y \oplus \gamma)}(B),
\end{aligned}
$$

onde (5.3) e (5.5) seguem de (5.1) e de (5.2); e (5.4) segue de $x>0$ e da hipótese de $v \mathrm{~A}_{\gamma}(A) \leq$ $v \mathrm{~A}_{\gamma}(B)$. Logo, se $v \mathrm{~A}_{\gamma}(A) \leq v \mathrm{~A}_{\gamma}(B)$, então $v \mathrm{~A}_{x \otimes(y \oplus \gamma)}(A) \leq v \mathrm{~A}_{x \otimes(y \oplus \gamma)}(B)$. Reciprocamente, suponha que $v \mathrm{~A}_{x \otimes(y \oplus \gamma)}(A) \leq v \mathrm{~A}_{x \otimes(y \oplus \gamma)}(B)$. Segue novamente de (5.1) e (5.2) que

$$
\begin{aligned}
x\left(y(n+m)+v \mathrm{~A}_{\gamma}(A)\right) & =v \mathrm{~A}_{x \otimes(y \oplus \gamma)}(A) \\
& \leq v \mathrm{~A}_{x \otimes(y \oplus \gamma)}(B), \\
& =x\left(y(n+m)+v \mathrm{~A}_{\gamma}(B)\right),
\end{aligned}
$$

o que implica, sendo $x>0$, que $v \mathrm{~A}_{\gamma}(A) \leq v \mathrm{~A}_{\gamma}(B)$. Logo, se $v \mathrm{~A}_{x \otimes(y \oplus \gamma)}(A) \leq v \mathrm{~A}_{x \otimes(y \oplus \gamma)}(B)$, então $v \mathrm{~A}_{\gamma}(A) \leq v \mathrm{~A}_{\gamma}(B)$.

\subsection{Condições necessárias para $\gamma \sim \delta$ em $\mathbb{B}$}

Como consequência do Fato 71 e do Lema 72, ser gerada por uma matriz de pontuação $\gamma$ é condição suficiente para $\delta \sim \gamma$. Nesta seção mostramos que, para uma classe de matrizes de pontuação $\mathbb{B}$ que definimos a seguir, essa condição é necessária para $\gamma \sim \delta$. Mais do que isto, mostramos como determinar $x>0$ e $y$ tais que $\delta=x \otimes(y \oplus \gamma)$ quando $\gamma \sim \delta$.

Seja $\gamma$ uma matriz de pontuação. Dizemos que $\gamma$ é uma matriz inteira (racional, real) se todas as entradas de $\gamma$ são números inteiros (racionais, reais). Definimos a classe de matrizes de pontuação $\mathbb{B}$ como sendo aquela tal que se $\gamma \in \mathbb{B}$, então

1. $\gamma$ é racional,

2. $\gamma$ é simétrica,

3. $\gamma_{\mathrm{a} \rightarrow \mathrm{a}}=\gamma_{\mathrm{b} \rightarrow \mathrm{b}} \leq \gamma_{\mathrm{a} \rightarrow \mathrm{b}}$, para cada $\mathrm{a}, \mathrm{b} \in \Sigma \mathrm{e}$

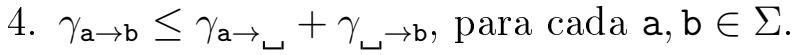

Primeiramente verificamos que as operações $\otimes$ e $\oplus$ são fechadas em $\mathbb{B}$.

Lema 73. Sejam $\gamma \in \mathbb{B}$ uma matriz de pontuação, $x, y \in \mathbb{Q}$, com $x>0$. Então, $x \otimes \gamma \mathrm{e}$ $y \oplus \gamma$ são elementos de $\mathbb{B}$.

Prova. Sejam a, $\mathrm{b} \in \Sigma$.

Temos que $x \cdot \gamma_{\mathrm{a} \rightarrow \mathrm{b}}=(x \otimes \gamma)_{\mathrm{a} \rightarrow \mathrm{b}}, x \cdot \gamma_{\mathrm{a} \rightarrow\lrcorner}=(x \otimes \gamma)_{\mathrm{a} \rightarrow_{\llcorner}}$e $x \cdot \gamma_{\mathrm{u}_{\mathrm{a}}}=(x \otimes \gamma)_{\mathrm{b}_{\mathrm{a}}}$ são números racionais pois o resultado da multiplicação de números racionais é um número racional. Ademais, $2 y+\gamma_{\mathrm{a} \rightarrow \mathrm{b}}=(y \oplus \gamma)_{\mathrm{a} \rightarrow \mathrm{b}}, y+\gamma_{\mathrm{a} \rightarrow \mathrm{b}}=(y \oplus \gamma)_{\mathrm{a} \rightarrow \mathrm{b}}$ e $y+\gamma_{\mathrm{u} \rightarrow \mathrm{a}}=(y \oplus \gamma)_{\rightarrow \mathrm{a}}$ são números racionais pois a soma de números racionais também é um número racional. 
Como $\gamma_{\mathrm{a} \rightarrow \mathrm{b}}=\gamma_{\mathrm{b} \rightarrow \mathrm{a}}$ e $\gamma_{\mathrm{a} \rightarrow_{\sqcup}}=\gamma_{\llcorner\rightarrow \mathrm{a}}$, temos que

$$
\begin{aligned}
& (x \otimes \gamma)_{\mathrm{a} \rightarrow \mathrm{b}}=x \cdot \gamma_{\mathrm{a} \rightarrow \mathrm{b}}=x \cdot \gamma_{\mathrm{b} \rightarrow \mathrm{a}}=(x \otimes \gamma)_{\mathrm{b} \rightarrow \mathrm{a}}, \\
& (x \otimes \gamma)_{\mathrm{a} \rightarrow \sqcup}=x \cdot \gamma_{\mathrm{a} \rightarrow \sqcup}=x \cdot \gamma_{\mathrm{u} \rightarrow \mathrm{a}}=(x \otimes \gamma)_{\mathrm{u} \rightarrow \mathrm{a}} \text {, } \\
& (y \oplus \gamma)_{\mathrm{a} \rightarrow \mathrm{b}}=2 y+\gamma_{\mathrm{a} \rightarrow \mathrm{b}}=2 y+\gamma_{\mathrm{b} \rightarrow \mathrm{a}}=(y \oplus \gamma)_{\mathrm{b} \rightarrow \mathrm{a}} \text {, }
\end{aligned}
$$

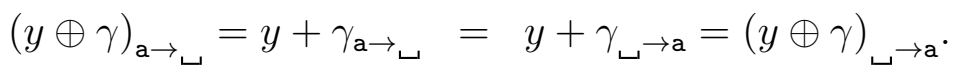

Como $\gamma_{\mathrm{a} \rightarrow \mathrm{a}}=\gamma_{\mathrm{b} \rightarrow \mathrm{b}} \leq \gamma_{\mathrm{a} \rightarrow \mathrm{b}}$ e $x>0$, temos que

$$
\begin{gathered}
(x \otimes \gamma)_{\mathrm{a} \rightarrow \mathrm{a}}=(x \otimes \gamma)_{\mathrm{b} \rightarrow \mathrm{b}}=x \cdot \gamma_{\mathrm{a} \rightarrow \mathrm{a}}=x \cdot \gamma_{\mathrm{b} \rightarrow \mathrm{b}} \leq x \cdot \gamma_{\mathrm{a} \rightarrow \mathrm{b}}=(x \otimes \gamma)_{\mathrm{a} \rightarrow \mathrm{b}} \\
(y \oplus \gamma)_{\mathrm{a} \rightarrow \mathrm{a}}=(y \oplus \gamma)_{\mathrm{b} \rightarrow \mathrm{b}}=2 y+\gamma_{\mathrm{a} \rightarrow \mathrm{a}}=2 y+\gamma_{\mathrm{b} \rightarrow \mathrm{b}} \leq 2 y+\gamma_{\mathrm{a} \rightarrow \mathrm{b}}=(x \oplus \gamma)_{\mathrm{a} \rightarrow \mathrm{b}} .
\end{gathered}
$$

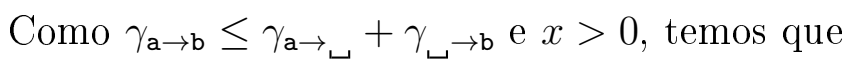

$$
\begin{aligned}
& (x \otimes \gamma)_{\mathrm{a} \rightarrow \mathrm{b}}=x \cdot \gamma_{\mathrm{a} \rightarrow \mathrm{b}} \leq x \cdot \gamma_{\mathrm{a} \rightarrow \mathrm{b}}+x \cdot \gamma_{\mathrm{c} \rightarrow \mathrm{b}}=(x \otimes \gamma)_{\mathrm{a} \rightarrow_{\mathrm{b}}}+(x \otimes \gamma)_{\mathrm{u} \rightarrow \mathrm{b}},
\end{aligned}
$$

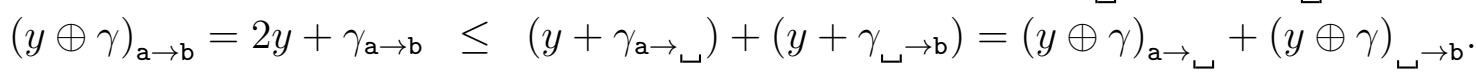

Desde que as propriedades acima são válidas para os símbolos arbitrários a e b, segue que as entradas das matrizes $x \otimes \gamma$ e $y \oplus \gamma$ são números racionais; $x \otimes \gamma$ e $y \oplus \gamma$ são simétricas; $(x \otimes \gamma)_{\mathrm{a} \rightarrow \mathrm{a}}=(x \otimes \gamma)_{\mathrm{b} \rightarrow \mathrm{b}} \leq(x \otimes \gamma)_{\mathrm{a} \rightarrow \mathrm{b}} \mathrm{e}(y \oplus \gamma)_{\mathrm{a} \rightarrow \mathrm{a}}=(y \oplus \gamma)_{\mathrm{b} \rightarrow \mathrm{b}} \leq(y \oplus \gamma)_{\mathrm{a} \rightarrow \mathrm{b}}$, $\mathrm{e}(x \otimes \gamma)_{\mathrm{a} \rightarrow \mathrm{b}} \leq(x \otimes \gamma)_{\mathrm{a}_{\llcorner}}+(x \otimes \gamma)_{\llcorner\rightarrow \mathrm{b}} \mathrm{e}(y \oplus \gamma)_{\mathrm{a} \rightarrow \mathrm{b}} \leq(y \oplus \gamma)_{\mathrm{a} \rightarrow \sqcup}+(y \oplus \gamma)_{{ } \rightarrow \mathrm{b}}$ para cada $\mathrm{a}, \mathrm{b} \in \Sigma$. Segue daí que $x \otimes \gamma, y \oplus \gamma \in \vec{\in} \mathbb{B}$.

Definimos $m d c(\gamma)$ como o maior inteiro positivo capaz de dividir cada elemento de uma matriz inteira $\gamma \neq 0$, ou seja, o maior inteiro $x$ tal que $(1 / x) \otimes \gamma$ é uma matriz inteira.

Fato 74. Seja $\gamma \neq 0$ uma matriz de pontuação racional. Existe um número racional $x>0$ tal que $x \otimes \gamma$ é uma matriz inteira e $m d c(x \otimes \gamma)=1$.

Seja $\mathcal{B}$ o conjunto das matrizes de pontuação $\gamma$ que possuem as seguintes propriedades:

$P_{0} . \gamma \in \mathbb{B}$

$P_{1} . \gamma_{\mathrm{a} \rightarrow \mathrm{a}}=0$ para algum $\mathrm{a} \in \Sigma$,

$P_{2}$. $\gamma$ é uma matriz inteira e

$P_{3} \cdot \gamma=0$ ou $\operatorname{mdc}(\gamma)=1$.

Como consequência dos Itens 3 e 4 da definição de $\mathbb{B}$ e de $P_{1}$ da definição de $\mathcal{B}$, temos que matrizes em $\mathcal{B}$ não possuem entradas negativas. Além disso, também como consequência do Item 3 da definição de $\mathbb{B}$ e de $P_{1}$ da definição de $\mathcal{B}$, temos que, se $\gamma \in \mathcal{B}$, então $\gamma_{a \rightarrow a}=0$, para cada a $\in \Sigma$. Também como consequência da definição, $\gamma=0 \in \mathcal{B}$.

O seguinte resultado mostra que toda matriz de pontuação em $\mathbb{B}$ possui uma matriz de pontuação equivalente em $\mathcal{B}$.

Lema 75. Sejam $\gamma \in \mathbb{B}$ e a $\in \Sigma$. Então, para $y=-\gamma_{\mathrm{a} \rightarrow \mathrm{a}} / 2$, existe um número racional positivo $x$ tal que

$$
x \otimes(y \oplus \gamma) \in \mathcal{B},
$$

e $x \otimes(y \oplus \gamma)$ é uma matriz inteira e $y \oplus \gamma=0$ ou $m d c(x \otimes(y \oplus \gamma))=1$. 
Prova. Como $\gamma \in \mathbb{B}$, por definição temos que $y=-\gamma_{a \rightarrow a} / 2$ é um número racional. Segue do Lema 73 que a matriz de pontuação $\delta=y \oplus \gamma \in \mathbb{B}$. Note que $\delta_{\mathrm{a} \rightarrow \mathrm{a}}=2\left(-\gamma_{\mathrm{a} \rightarrow \mathrm{a}} / 2\right)+\gamma_{\mathrm{a} \rightarrow \mathrm{a}}=0$. Logo, desde que $\delta \in \mathbb{B}$, temos que $\delta_{\mathrm{b} \rightarrow \mathrm{b}}=0$ para cada $\mathrm{b} \in \Sigma$.

Suponha $\delta=0$. Então $1 \otimes(y \oplus \gamma)=1 \otimes \delta=0 \in \mathcal{B}$ e o lema está provado. Assumimos então que $\delta \neq 0$.

Como $\delta \in \mathbb{B}$, por definição temos que as entradas de $\delta$ são números racionais. Segue do Fato 74 , sendo $\delta \neq 0$, que existe um racional $x>0$ tal que a matriz $\varphi=x \otimes \delta$ tem entradas inteiras e $\operatorname{mdc}(\varphi)=1$. Temos também pelo Lema 73, desde que $\delta \in \mathbb{B}$ e $x$ é um racional positivo, que $\varphi \in \mathbb{B}$. Temos também, desde que $\delta_{\mathrm{a} \rightarrow \mathrm{a}}=0$, que $\varphi_{\mathrm{a} \rightarrow \mathrm{a}}=x \cdot \delta_{\mathrm{a} \rightarrow \mathrm{a}}=0$. Segue das observações deste parágrafo que

$$
x \otimes(y \oplus \gamma)=\varphi \in \mathcal{B} .
$$

Se $\gamma, \delta \in \mathcal{B}$ são tais que $\gamma, \delta \neq 0$, então por definição $m d c(\gamma)=m d c(\delta)=1$. Como consequência, temos o seguinte fato.

Fato 76. Sejam $\gamma, \delta \in \mathcal{B}$ tais que $\gamma \neq 0, \delta \neq 0$ e $x>0$. Se $x \otimes \gamma=\delta$, então $x=1$ e, portanto, $\gamma=\delta$.

As proposições a seguir são resultados intermediários fracos. Entretanto, eles auxiliam a demonstração do Lema 82.

Proposição 77. Sejam $\gamma, \delta \in \mathcal{B}, \gamma \sim \delta$ e a $\in \Sigma$. Se $\gamma_{\mathrm{a} \rightarrow_{\sqcup}}=0$, então $\delta_{\mathrm{a} \rightarrow_{\sqcup}}=0$.

Prova. Suponha que $\gamma_{a \rightarrow \sqcup}=0$. Como $\gamma, \delta \in \mathcal{B}$, temos que $\gamma_{a \rightarrow a}=\delta_{a \rightarrow a}=0, \gamma_{a} \rightarrow \sqcup=\gamma_{\sqcup \rightarrow a}$

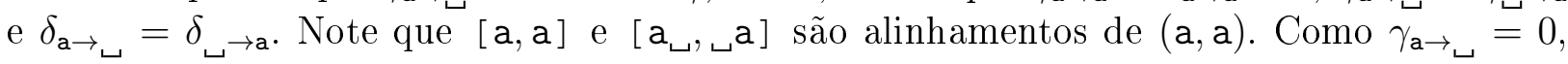
$\gamma_{\mathrm{a} \rightarrow \mathrm{a}}=0$ e $\gamma_{\mathrm{a} \rightarrow \mathrm{b}}=\gamma_{\mathrm{u} \rightarrow \mathrm{a}}$, temos que

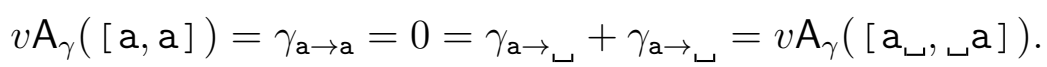

Como $\gamma \sim \delta$ e $v \mathrm{~A}_{\gamma}([\mathrm{a}, \mathrm{a}])=v \mathrm{~A}_{\gamma}\left(\left[\mathrm{a}_{\sqcup},{ }^{\mathrm{a}} \mathrm{a}\right]\right)$, temos que $v \mathrm{~A}_{\delta}([\mathrm{a}, \mathrm{a}])=v \mathrm{~A}_{\delta}\left(\left[\mathrm{a}_{\sqcup},{ }^{\mathrm{a}}\right]\right), \mathrm{o}$ que implica, sendo $\delta_{\mathrm{a} \rightarrow \mathrm{a}}=0$, que

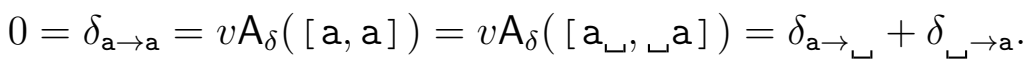

Como $\delta_{\mathrm{a} \rightarrow_{\sqcup}}+\delta_{\iota \rightarrow \mathrm{a}}=0$ e $\delta_{\mathrm{a} \rightarrow_{\sqcup}}=\delta_{\sqcup \rightarrow \mathrm{a}}$, temos que

$$
\delta_{\mathrm{a} \rightarrow_{\sqcup}}=\frac{1}{2}\left(\delta_{\mathrm{a} \rightarrow_{\sqcup}}+\delta_{\mathrm{a} \rightarrow_{\sqcup}}\right)=\frac{1}{2}\left(\delta_{\mathrm{a} \rightarrow_{\sqcup}}+\delta_{\sqcup \rightarrow \mathrm{a}}\right)=\frac{1}{2}(0)=0 .
$$

Portanto, se $\gamma_{\mathrm{a} \rightarrow_{\sqcup}}=0$, então $\delta_{\mathrm{a} \rightarrow_{\iota}}=0$.

Proposição 78. Sejam $\gamma \in \mathcal{B}$ e a, b $\in \Sigma$. Se $\gamma_{\mathrm{a} \rightarrow_{\sqcup}}=\gamma_{\mathrm{b} \rightarrow \sqcup}=0$, então $\gamma_{\mathrm{a} \rightarrow \mathrm{b}}=0$.

Prova. Suponha $\gamma_{\mathrm{a} \rightarrow_{\sqcup}}=\gamma_{\mathrm{b} \rightarrow_{\sqcup}}=0$. Como $\gamma \in \mathcal{B}$, temos que $\gamma_{\mathrm{a} \rightarrow \mathrm{b}}$ é não negativo e que $\gamma_{\mathrm{a} \rightarrow \mathrm{b}} \leq \gamma_{\mathrm{a} \rightarrow \sqcup}+\gamma_{\sqcup \rightarrow \mathrm{b}}$ e $\gamma_{\llcorner\rightarrow \mathrm{b}}=\gamma_{\mathrm{b} \rightarrow \sqcup}$. Segue que

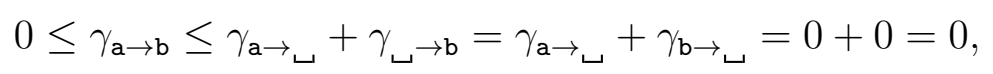

o que implica que $\gamma_{\mathrm{a} \rightarrow \mathrm{b}}=0$. Portanto, se $\gamma_{\mathrm{a} \rightarrow_{\sqcup}}=\gamma_{\mathrm{b} \rightarrow_{\sqcup}}=0$, então $\gamma_{\mathrm{a} \rightarrow \mathrm{b}}=0$. 
Proposição 79. Seja $\gamma \in \mathcal{B}$. Se $\gamma_{\mathrm{a} \rightarrow_{\sqcup}}=0$ para cada $\mathrm{a} \in \Sigma$, então $\gamma=0$.

Prova. Sejam a, $\mathrm{b} \in \Sigma$ e suponha que $\gamma_{\mathrm{a} \rightarrow_{\llcorner}}=\gamma_{\mathrm{b} \rightarrow_{\llcorner}}=0$. Desde que $\gamma \in \mathcal{B} \subseteq \mathbb{B}$, segue que $\gamma_{\lrcorner \rightarrow b}=\gamma_{b \rightarrow \sqcup}=0$ e $\gamma_{\lrcorner \rightarrow a}=\gamma_{a \rightarrow \sqcup}=0$. Além disso, segue da Proposição 78 que $\gamma_{\mathrm{a} \rightarrow \mathrm{b}}=0$. Desde que $\gamma_{\llcorner\rightarrow \mathrm{a}}=\gamma_{\mathrm{a} \rightarrow \sqcup}=\gamma_{\llcorner\rightarrow \mathrm{b}}=\gamma_{\mathrm{b} \rightarrow\llcorner}=\gamma_{\mathrm{a} \rightarrow \mathrm{b}}=0$ para os símbolos arbitrários $\mathrm{a}, \mathrm{b} \in \Sigma$, segue que se $\gamma_{\mathrm{a} \rightarrow \sqcup}=0$ para cada a $\in \Sigma$, então $\gamma=0$.

Proposição 80. Sejam $\gamma, \delta \in \mathcal{B}$ e a, b $\in \Sigma$. Se $\gamma \sim \delta$, então $\gamma_{\mathrm{b} \rightarrow \sqcup} \delta_{\mathrm{a} \rightarrow \sqcup}=\gamma_{\mathrm{a} \rightarrow \sqcup} \delta_{\mathrm{b} \rightarrow \sqcup}$.

Prova. Sejam os inteiros $y=\gamma_{\mathrm{a} \rightarrow_{\sqcup}} \geq 0$ e $z=\gamma_{\mathrm{b} \rightarrow_{\sqcup}} \geq 0$, e os alinhamentos

$$
A=\left[\begin{array}{ccc}
\mathrm{a}^{z} & \mathrm{~b}^{y} & \sqcup^{z} \\
z & \mathrm{~b}^{y} & \mathrm{a}^{z}
\end{array}\right] \text { e } B=\left[\begin{array}{rrr}
\sqcup^{y} & \mathrm{a}^{z} & \mathrm{~b}^{y} \\
\mathrm{~b}^{y} & \mathrm{a}^{z} & \sqcup^{y}
\end{array}\right]
$$

de $\left(\mathrm{a}^{z} \mathrm{~b}^{y}, \mathrm{~b}^{y} \mathrm{a}^{z}\right)$. Como $\gamma \in \mathcal{B}$, temos que $\gamma_{\mathrm{a} \rightarrow\lrcorner}=\gamma_{\lrcorner \rightarrow \mathrm{a}}, \gamma_{\mathrm{b} \rightarrow_{\lrcorner}}=\gamma_{\lrcorner \rightarrow \mathrm{b}}$ e $\gamma_{\mathrm{a} \rightarrow \mathrm{a}}=\gamma_{\mathrm{b} \rightarrow \mathrm{b}}=0$. Logo,

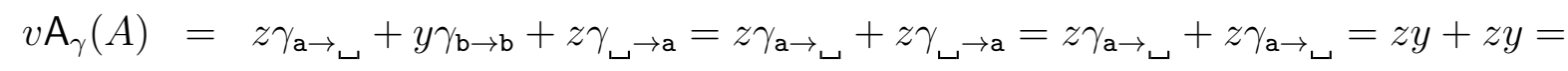

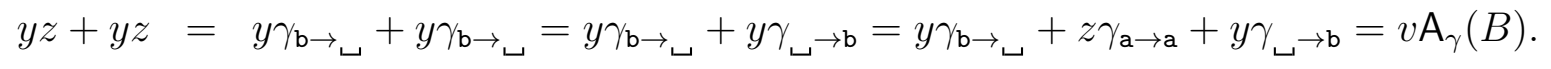

Suponha que $\gamma \sim \delta$. Desde que $v \mathrm{~A}_{\gamma}(A)=v \mathrm{~A}_{\gamma}(B)$, segue que $v \mathrm{~A}_{\delta}(A)=v \mathrm{~A}_{\delta}(B)$. Como

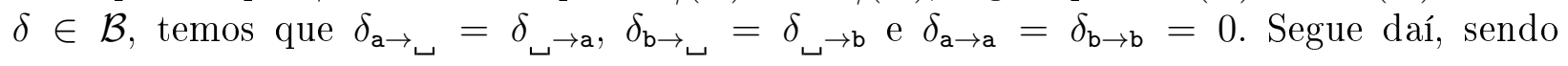
$y=\gamma_{\mathrm{a} \rightarrow \sqcup}=\gamma_{\sqcup \rightarrow \mathrm{a}}$ e $z=\gamma_{\mathrm{b} \rightarrow \sqcup}=\gamma_{\sqcup \rightarrow \mathrm{b}}$, que

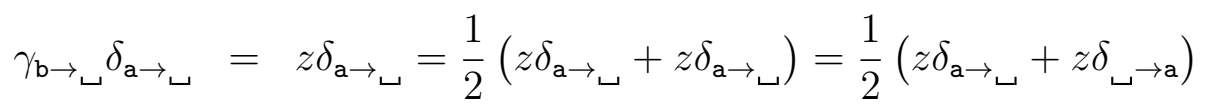

$$
\begin{aligned}
& =\frac{1}{2}\left(z \delta_{\mathrm{a} \rightarrow \sqcup}+y \delta_{\mathrm{b} \rightarrow \mathrm{b}}+z \delta_{\sqcup \rightarrow \mathrm{a}}\right)=\frac{1}{2} v \mathrm{~A}_{\delta}(A) \\
& =\frac{1}{2} v \mathrm{~A}_{\delta}(B)=\frac{1}{2}\left(y \delta_{\sqcup \rightarrow \mathrm{b}}+z \delta_{\mathrm{a} \rightarrow \mathrm{a}}+y \delta_{\mathrm{b} \rightarrow}\right)
\end{aligned}
$$

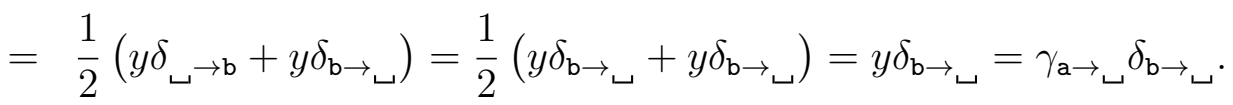

Portanto, se $\gamma \sim \delta$, então $\gamma_{\mathrm{b} \rightarrow} \delta_{\mathrm{a} \rightarrow \sqcup}=\gamma_{\mathrm{a} \rightarrow \sqcup} \delta_{\mathrm{b} \rightarrow_{\sqcup}}$.

Proposição 81. Sejam $\gamma, \delta \in \mathcal{B}$ e a, b $\in \Sigma$. Se $\gamma \sim \delta$, então $\delta_{\mathrm{a} \rightarrow\lrcorner} \gamma_{\mathrm{a} \rightarrow \mathrm{b}}=\gamma_{\mathrm{a} \rightarrow} \delta_{\mathrm{a} \rightarrow \mathrm{b}}$.

Prova. Suponha que $\gamma \sim \delta$. Se $\gamma_{\mathrm{a} \rightarrow \sqcup}=0$, então segue da Proposição 77 que $\delta_{\mathrm{a} \rightarrow \sqcup}=0$ e, neste caso, $\delta_{\mathrm{a} \rightarrow\llcorner} \gamma_{\mathrm{a} \rightarrow \mathrm{b}}=0=\gamma_{\mathrm{a} \rightarrow\llcorner} \delta_{\mathrm{a} \rightarrow \mathrm{b}}$ e a prova está feita. Assumimos então que $\gamma_{\mathrm{a} \rightarrow \sqcup} \neq 0$. Como $\gamma \in \mathcal{B}$, temos que as entradas de $\gamma$ são não negativas. Segue que $\gamma_{a \rightarrow}>0$.

Considere, sendo $\gamma \in \mathcal{B}$, os inteiros não negativos

$$
y=\gamma_{\mathrm{a} \rightarrow \sqcup}, \quad z=\gamma_{\mathrm{b} \rightarrow \sqcup} \text { e } x=\gamma_{\mathrm{a} \rightarrow \mathrm{b}}
$$

Sejam os alinhamentos

$$
\begin{aligned}
& A=\left[\left(\begin{array}{ll}
\mathrm{a} & \mathrm{b} \\
\mathrm{b} & \mathrm{b}
\end{array}\right)^{x}\left(\begin{array}{ll}
\mathrm{a} & \mathrm{b} \\
\mathrm{b} & \mathrm{a}
\end{array}\right)^{y+z-x}\left(\begin{array}{ll}
\mathrm{a} & \mathrm{a} \\
\mathrm{b} & \mathrm{a}
\end{array}\right)^{x} \mathrm{~b}\right] \mathrm{e}
\end{aligned}
$$

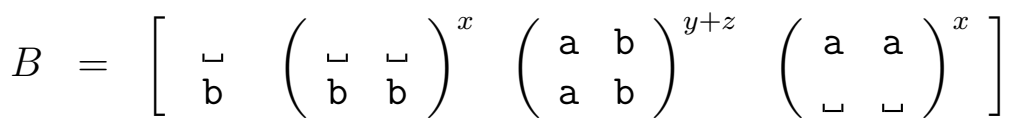

de $\left((\mathrm{ab})^{y+z}(\mathrm{aa})^{x}, \mathrm{~b}(\mathrm{bb})^{x}(\mathrm{ab})^{y+z}\right)$. Note que da definição de $\mathcal{B}$ temos que $y+z-x=\gamma_{\mathrm{a} \rightarrow}+$ $\gamma_{\mathrm{b} \rightarrow\llcorner}-\gamma_{\mathrm{a} \rightarrow \mathrm{b}} \geq 0$. 
Como $\gamma \in \mathcal{B}$, temos que $\gamma_{\mathrm{b} \rightarrow \mathrm{a}}=\gamma_{\mathrm{a} \rightarrow \mathrm{b}}, \gamma_{\llcorner\rightarrow \mathrm{b}}=\gamma_{\mathrm{b} \rightarrow \sqcup}$ e $\gamma_{\mathrm{a} \rightarrow \mathrm{a}}=\gamma_{\mathrm{b} \rightarrow \mathrm{b}}=0$. Segue daí que

$$
\begin{aligned}
& v \mathrm{~A}_{\gamma}(A)=x\left(\gamma_{\mathrm{a} \rightarrow \mathrm{b}}+\gamma_{\mathrm{b} \rightarrow \mathrm{b}}\right)+(y+z-x)\left(\gamma_{\mathrm{a} \rightarrow \mathrm{b}}+\gamma_{\mathrm{b} \rightarrow \mathrm{a}}\right)+x\left(\gamma_{\mathrm{a} \rightarrow \mathrm{b}}+\gamma_{\mathrm{a} \rightarrow \mathrm{a}}\right)+\gamma_{\mathrm{s} \rightarrow \mathrm{b}} \\
& =x \gamma_{\mathrm{a} \rightarrow \mathrm{b}}+(y+z-x)\left(\gamma_{\mathrm{a} \rightarrow \mathrm{b}}+\gamma_{\mathrm{a} \rightarrow \mathrm{b}}\right)+x \gamma_{\mathrm{a} \rightarrow \mathrm{b}}+\gamma_{\mathrm{c} \rightarrow \mathrm{b}} \\
& =2(y+z) \gamma_{\mathrm{a} \rightarrow \mathrm{b}}+\gamma_{\mathrm{c} \rightarrow \mathrm{b}} \\
& =2 x\left(\gamma_{\mathrm{a} \rightarrow_{\sqcup}}+\gamma_{\mathrm{b} \rightarrow \sqcup}\right)+\gamma_{\sqcup \rightarrow \mathrm{b}} \\
& =2 x\left(\gamma_{\mathrm{a} \rightarrow \boldsymbol{\sqcup}}+\gamma_{\boldsymbol{U} \rightarrow \mathrm{b}}\right)+\gamma_{\mathbf{L}} \rightarrow \mathrm{b}
\end{aligned}
$$

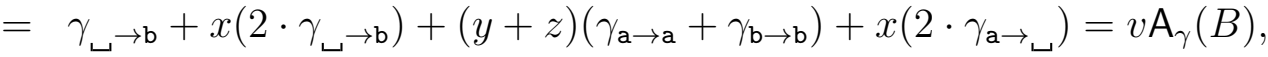

o que implica, sendo $\gamma \sim \delta$, que $v \mathrm{~A}_{\delta}(A)=v \mathrm{~A}_{\delta}(B)$. Como $\delta \in \mathcal{B}$, temos que $\delta_{\mathrm{b} \rightarrow \mathrm{a}}=\delta_{\mathrm{a} \rightarrow \mathrm{b}}$,

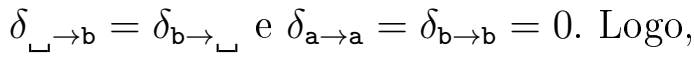

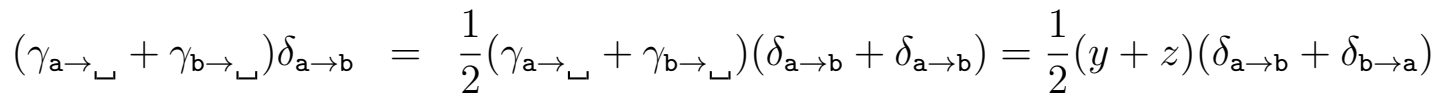

$$
\begin{aligned}
& =\frac{(y+z)\left(\delta_{\mathrm{a} \rightarrow \mathrm{b}}+\delta_{\mathrm{b} \rightarrow \mathrm{a}}\right)+\delta_{\mathrm{u} \rightarrow \mathrm{b}}-\delta_{\mathrm{u} \rightarrow \mathrm{b}}+x \cdot \delta_{\mathrm{b} \rightarrow \mathrm{b}}+x \cdot \delta_{\mathrm{a} \rightarrow \mathrm{a}}}{2} \\
& =\frac{v \mathrm{~A}_{\delta}(A)-\delta_{\boldsymbol{\omega}} \rightarrow \mathrm{b}}{2}=\frac{v \mathrm{~A}_{\delta}(B)-\delta_{\boldsymbol{U}} \rightarrow \mathrm{b}}{2} \\
& =\frac{(2 x+1) \delta_{\mathrm{u} \rightarrow \mathrm{b}}+(y+z)\left(\delta_{\mathrm{a} \rightarrow \mathrm{a}}+\delta_{\mathrm{b} \rightarrow \mathrm{b}}\right)+2 x \cdot \delta_{\mathrm{a} \rightarrow \mathrm{u}}-\delta_{\mathrm{u} \rightarrow \mathrm{b}}}{2} \\
& =x\left(\delta_{\mathrm{a} \rightarrow_{\sqcup}}+\delta_{\iota^{\rightarrow} \mathrm{b}}\right)=x\left(\delta_{\mathrm{a} \rightarrow_{\sqcup}}+\delta_{\mathrm{b} \rightarrow_{\sqcup}}\right) \\
& =\gamma_{\mathrm{a} \rightarrow \mathrm{b}}\left(\delta_{\mathrm{a} \rightarrow_{\sqcup}}+\delta_{\mathrm{b} \rightarrow_{\sqcup}}\right) \text {. }
\end{aligned}
$$

Então, desde que $\gamma \in \mathcal{B}$, temos que $\gamma_{\mathrm{b} \rightarrow_{\sqcup}} \geq 0$. Além disso, por hipótese $\gamma_{\mathrm{a} \rightarrow_{\sqcup}}>0$. Logo, $\gamma_{a \rightarrow \sqcup}+\gamma_{b \rightarrow \sqcup}>0$. Segue que

$$
\begin{aligned}
& \delta_{\mathrm{a} \rightarrow_{\sqcup}} \gamma_{\mathrm{a} \rightarrow \mathrm{b}}=\left(\frac{\gamma_{\mathrm{a} \rightarrow_{\sqcup}}+\gamma_{\mathrm{b} \rightarrow_{\sqcup}}}{\gamma_{\mathrm{a} \rightarrow_{\sqcup}}+\gamma_{\mathrm{b} \rightarrow_{\sqcup}}}\right) \delta_{\mathrm{a} \rightarrow_{\sqcup}} \gamma_{\mathrm{a} \rightarrow \mathrm{b}} \\
& =\left(\frac{\gamma_{a \rightarrow \sqcup} \delta_{a \rightarrow \sqcup}+\gamma_{b \rightarrow} \delta_{a \rightarrow \sqcup}}{\gamma_{a \rightarrow \sqcup}+\gamma_{b \rightarrow \sqcup}}\right) \gamma_{a \rightarrow b} \\
& =\left(\frac{\gamma_{a \rightarrow} \delta_{a \rightarrow \sqcup}+\gamma_{a \rightarrow \sqcup} \delta_{b \rightarrow \sqcup}}{\gamma_{a \rightarrow \sqcup}+\gamma_{b \rightarrow \sqcup}}\right) \gamma_{a \rightarrow b} \\
& =\frac{\gamma_{\mathrm{a} \rightarrow \sqcup}}{\gamma_{\mathrm{a} \rightarrow \sqcup}+\gamma_{\mathrm{b} \rightarrow \sqcup}}\left(\delta_{\mathrm{a} \rightarrow_{\sqcup}}+\delta_{\mathrm{b} \rightarrow \sqcup}\right) \gamma_{\mathrm{a} \rightarrow \mathrm{b}} \\
& =\frac{\gamma_{a \rightarrow \sqcup}}{\gamma_{a \rightarrow \sqcup}+\gamma_{b \rightarrow \sqcup}}\left(\gamma_{a \rightarrow \sqcup}+\gamma_{b \rightarrow \sqcup}\right) \delta_{a \rightarrow b} \\
& =\gamma_{\mathrm{a} \rightarrow \sqcup} \delta_{\mathrm{a} \rightarrow \mathrm{b}} \text {. }
\end{aligned}
$$

onde (5.7) segue da Proposição 80 e (5.8) segue de (5.6). Concluímos que se $\gamma \sim \delta$, então $\delta_{\mathrm{a} \rightarrow\llcorner} \gamma_{\mathrm{a} \rightarrow \mathrm{b}}=\gamma_{\mathrm{a} \rightarrow\llcorner} \delta_{\mathrm{a} \rightarrow \mathrm{b}}$.

Lema 82. Sejam $\gamma, \delta \in \mathcal{B}$. Se $\gamma \sim \delta$, então $\gamma=\delta$.

Prova. Suponha que $\gamma \sim \delta$. Se $\gamma_{\mathrm{a} \rightarrow_{\sqcup}}=0$ para cada $\mathrm{a} \in \Sigma$, então pela Proposição 77 segue que $\delta_{\mathrm{a} \rightarrow\lrcorner}=0$ para cada $\mathrm{a} \in \Sigma$. Segue então da Proposição 79 que $\gamma=0$ e $\delta=0$ e a prova está feita. Assumimos então que existe a $\in \Sigma, \gamma_{a \rightarrow_{\llcorner}} \neq 0$. Seja $k=\delta_{a \rightarrow_{\iota}} / \gamma_{a \rightarrow_{\sqcup}}$.

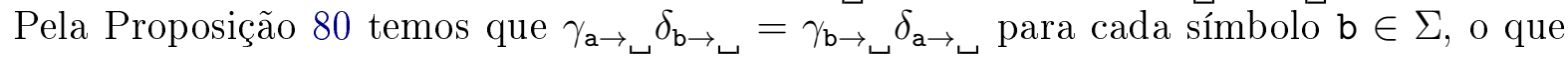


implica, sendo $\gamma_{\mathrm{a} \rightarrow_{\sqcup}} \neq 0$, que

$$
\delta_{\mathrm{b} \rightarrow \sqcup}=\frac{\delta_{\mathrm{a} \rightarrow \sqcup}}{\gamma_{\mathrm{a} \rightarrow \sqcup}} \gamma_{\mathrm{b} \rightarrow \sqcup}=k \gamma_{\mathrm{b} \rightarrow \sqcup} .
$$

Além disso, como $\gamma, \delta \in \mathcal{B} \subseteq \mathbb{B}$, temos que $\gamma_{\mathrm{b} \rightarrow_{\sqcup}}=\gamma_{\iota^{\rightarrow b}}$ e $\delta_{\mathrm{b} \rightarrow_{\sqcup}}=\delta_{{ } \rightarrow \mathrm{b}}$. Segue que

$$
\delta_{\sqcup \rightarrow \mathrm{b}}=\delta_{\mathrm{b} \rightarrow \sqcup}=k \gamma_{\mathrm{b} \rightarrow \sqcup}=k \gamma_{\lrcorner \rightarrow \mathrm{b}},
$$

para cada símbolo $\mathrm{b} \in \Sigma$.

Sejam b, c $\in \Sigma$. Suponha que $\gamma_{\mathrm{b} \rightarrow_{\sqcup}}=\gamma_{\mathrm{c} \rightarrow_{\sqcup}}=0$. Segue da Proposição 77 que $\delta_{\mathrm{b} \rightarrow_{\sqcup}}=$ $\delta_{\mathrm{c} \rightarrow\lrcorner}=0$. Segue da Proposição 78 que $\gamma_{\mathrm{b} \rightarrow \mathrm{c}}=\delta_{\mathrm{b} \rightarrow \mathrm{c}}=0$. Logo, $\delta_{\mathrm{b} \rightarrow \mathrm{c}}=k \gamma_{\mathrm{b} \rightarrow \mathrm{c}}$. Assumimos então que $\gamma_{\mathrm{b} \rightarrow_{\sqcup}} \neq 0$ ou $\gamma_{\lrcorner \rightarrow \mathrm{c}} \neq 0$. Se $\gamma_{\mathrm{b} \rightarrow_{\sqcup}} \neq 0$, então, por (5.9), $\delta_{\mathrm{b} \rightarrow_{\sqcup}}=k \gamma_{\mathrm{b} \rightarrow \sqcup}$ e, pela Proposição 81, $\gamma_{\mathrm{b} \rightarrow \smile} \delta_{\mathrm{b} \rightarrow \mathrm{c}}=\delta_{\mathrm{b} \rightarrow\lrcorner} \gamma_{\mathrm{b} \rightarrow \mathrm{c}}$. Segue que

$$
\delta_{\mathrm{b} \rightarrow \mathrm{c}}=\frac{\delta_{\mathrm{b} \rightarrow{ }_{\sqcup}} \gamma_{\mathrm{b} \rightarrow \mathrm{c}}}{\gamma_{\mathrm{b} \rightarrow \sqcup}}=\frac{k \gamma_{\mathrm{b} \rightarrow} \gamma_{\mathrm{b} \rightarrow \mathrm{c}}}{\gamma_{\mathrm{b} \rightarrow \sqcup}}=k \gamma_{\mathrm{b} \rightarrow \mathrm{c}} .
$$

Similarmente, se $\gamma_{\mathrm{c} \rightarrow \sqcup} \neq 0$, então $\delta_{\mathrm{b} \rightarrow \mathrm{c}}=k \delta_{\mathrm{b} \rightarrow \mathrm{c}}$.

Pelos argumentos acima, concluímos que $\gamma=k \otimes \delta$, o que implica pelo Fato 76 que $\gamma=\delta$. Portanto, se $\gamma \sim \delta$, então $\gamma=\delta$.

Os resultados até aqui obtidos implicitamente sugerem um método para decidir se $\gamma \sim \delta$ quando $\gamma, \delta$ são matrizes de pontuação em $\mathbb{B}$. Como consequência do Lema 75 , temos que existem matrizes $\gamma^{\prime}, \delta^{\prime}$ em $\mathcal{B}$ tais que $\gamma^{\prime} \sim \gamma$ e $\delta^{\prime} \sim \delta$, e como consequência do Lema 82, desde que uma matriz é equivalente a ela mesma, temos que $\gamma \sim \delta$ se e somente se $\gamma^{\prime}=\delta^{\prime}$. O seguinte teorema e sua prova resumem esse resultado, caracterizando pares de matrizes de pontuação equivalentes em $\mathbb{B}$.

Teorema 83. Sejam $\gamma, \delta \in \mathbb{B}$. Então, $\delta$ é gerada por $\gamma$ se e somente se $\gamma \sim \delta$.

Prova. Para demonstrar o teorema mostramos que se $\delta$ é gerada por $\gamma$, então $\gamma \sim \delta$ e, depois, que se $\gamma \sim \delta$, então $\delta$ é gerada por $\gamma$.

Suponha que $\delta$ é gerada por $\gamma$. Pelo Fato 71 temos que existem números $x>0$ e $y$ tais que $\delta=x \otimes(y \oplus \gamma)$. Segue do Lema 72 que $\gamma \sim x \otimes(y \oplus \gamma)=\delta$. Portanto, se $\delta$ é gerada por $\gamma$, então $\gamma \sim \delta$.

Reciprocamente, suponha que $\gamma \sim \delta$. Desde que $\gamma, \delta \in \mathbb{B}$, pelo Lema 75 existem números $x_{1}>0, x_{2}>0, y_{1}$ e $y_{2}$ tais que $\gamma^{\prime}=x_{1} \otimes\left(y_{1} \oplus \gamma\right) \in \mathcal{B}$ e $\delta^{\prime}=x_{2} \otimes\left(y_{2} \oplus \delta\right) \in \mathcal{B}$. Segue do Lema 72 que $\gamma \sim \gamma^{\prime}$ e $\delta \sim \delta^{\prime}$, o que implica por simetria e transitividade, desde que $\gamma \sim \delta$, que $\gamma^{\prime} \sim \delta^{\prime}$. Segue do Lema 82 que $x_{1} \otimes\left(y_{1} \oplus \gamma\right)=\gamma^{\prime}=\delta^{\prime}=x_{2} \otimes\left(y_{2} \oplus \delta\right)$, o que implica usando as Propriedades P3 e P4, da página 75, que

$$
\gamma=-y_{1} \oplus\left(\frac{1}{x_{1}} \otimes\left(x_{2} \otimes\left(y_{2} \oplus \delta\right)\right)\right) .
$$

Portanto, se $\gamma \sim \delta$, então $\delta$ é gerada por $\gamma$. 


\section{Capítulo 6}

\section{Alinhamento de várias sequências}

Nesse capítulo definimos e analisamos novos critérios para, a partir de uma matriz $\gamma$, pontuar um alinhamento de uma $k$-upla de sequências normalizando a pontuação obtida para o critério $v \mathrm{SP}_{\gamma}$.

Em todos os critérios estudados o símbolo $\underset{\sqcup}{\sim}$ alinhado com $\_$não contribui diretamente para a definição da pontuação do alinhamento. Logo, não é necessária a definição da entrada $\gamma_{\llcorner\rightarrow}$. Entretanto, desde que os critérios estudados são aditivos e desde que não queremos mencionar em todo momento o fato de que [u, ] não contribui para a pontuação, consideramos que $\gamma_{\iota_{\lrcorner}}=0$.

\subsection{Introdução}

No Capítulo 2 vimos que

$$
v \mathrm{SP}_{\gamma}(A)=\sum_{h=1}^{k-1} \sum_{i=h+1}^{k} v \mathrm{~A}_{\gamma}\left(A_{\{h, i\}}\right)
$$

Propomos novos critérios para pontuar o alinhamento de várias sequências que levam em conta o "comprimento" dos alinhamentos. Como comprimento, no primeiro critério proposto $\left(\mathrm{V}_{\gamma}^{1}\right)$ consideramos o comprimento do alinhamento, no segundo $\left(\mathrm{V}_{\gamma}^{2}\right)$ consideramos o comprimento de cada alinhamento induzido e no terceiro $\left(\mathrm{V}_{\gamma}^{3}\right)$, a soma dos comprimentos dos alinhamentos induzidos. Esses critérios são formalizados mais precisamente a seguir:

$$
\begin{aligned}
& \mathrm{V}_{\gamma}^{1}(A)= \begin{cases}0 & \text { se }|A|=0 \\
v \operatorname{SP}_{\gamma}(A) /|A| & \text { caso contrário, }\end{cases} \\
& \mathrm{V}_{\gamma}^{2}(A)=\sum_{h=1}^{k-1} \sum_{i=h+1}^{k} v \mathrm{~N}_{\gamma}\left(A_{\{h, i\}}\right), \\
& \mathrm{V}_{\gamma}^{3}(A)= \begin{cases}0 & \text { se }|A|=0 \\
v \operatorname{SP}_{\gamma}(A) /\left(\sum_{h=1}^{k-1} \sum_{i=h+1}^{k}\left|A_{\{h, i\}}\right|\right) & \text { caso contrário. }\end{cases}
\end{aligned}
$$

Para uma $k$-upla de sequências $S$ e $\eta=1,2,3$, a função OPT ${ }_{\gamma}^{\eta}$ é definida para cada critério $\bigvee_{\gamma}^{\eta}$ como

$$
\mathrm{OPT}_{\gamma}^{\eta}(S)=\min _{A \in \mathcal{A}_{S}}\left\{\mathrm{~V}_{\gamma}^{\eta}(A)\right\}
$$

Dizemos que um alinhamento $A$ é $\bigvee_{\gamma}^{\eta}$-ótimo se $\bigvee_{\gamma}^{\eta}(A)=\operatorname{OPT}_{\gamma}^{\eta}(S)$. 


\subsection{Algoritmos exatos}

Seja $S=\left(s_{1}, \ldots, s_{k}\right) \in\left(\Sigma^{*}\right)^{k}$. Considere $n_{i}=\left|s_{i}\right|$ e $N=\sum_{i} n_{i}$. Para um conjunto $C$ qualquer, dizemos que uma sequência de $k$ elementos de $C$ é um $k$-vetor de elementos em $C$ e, assim como os alinhamentos, os $k$-vetores são denotados entre colchetes ("[" e "]"). Em geral dizemos $k$-vetores quando queremos nos referir a $k$-uplas que não são $k$-uplas de sequências de elementos em $\Sigma^{*}$ nem em $\Sigma^{*}$.

Sejam $\vec{v}=\left[v_{1}, v_{2}, \ldots, v_{k}\right]$ e $\vec{w}=\left[\vec{w}_{1}, w_{2}, \ldots, w_{k}\right] k$-vetores. Escrevemos $\vec{v}=\vec{w}$ se $v_{i}=w_{i}$ para todo $i$ e $\vec{v} \neq \vec{w}$ caso contrário. Se $\vec{v}$ e $\vec{w}$ são $k$-vetores de inteiros, escrevemos $\vec{v} \leq \vec{w}$ se $v_{i} \leq w_{i}$ para todo $i$; e $\vec{v}<\vec{w}$ se $\vec{v} \leq \vec{w}$ e existe $i$ tal que $v_{i}<w_{i}$. Denotamos $\vec{v}-\vec{w}$ o $k$-vetor $\left[v_{1}-w_{1}, \ldots, v_{k}-w_{k}\right]$.

Definimos $\vec{V}_{S}$ como sendo o conjunto dos $k$-vetores de inteiros $\left[v_{1}, \ldots, v_{k}\right]$ tais que $0 \leq v_{i} \leq n_{i}$ para cada $i$. Note que se $n_{i}=n$ para todo $i$, então $\left|\vec{V}_{S}\right|=(n+1)^{k}$. Denotamos por $S(\vec{v})$ a $k$-upla $\left(s_{1}\left(1 \ldots v_{1}\right), \ldots, s_{k}\left(1 \ldots v_{k}\right)\right)$ para $\vec{v} \in \vec{V}_{S}$, e dizemos que $S(\vec{v})$ é um prefixo de $S$. Um $k$-vetor de inteiros $\left[b_{1}, \ldots, b_{k}\right]$ é dito $k$-vetor binário se todo $b_{i} \in\{0,1\}$. Denotamos por $\mathbf{0}^{k}$ o $k$-vetor binário com todos os elementos da sequência iguais a zero, e o conjunto de todos os $k$-vetores binários diferentes de $\mathbf{0}^{k}$ por $\mathcal{B}_{k}$. Note, portanto, que $\left|\mathcal{B}_{k}\right|=2^{k}-1$.

Seja $\vec{b} \in \mathcal{B}_{k}$. Definimos $\vec{b} \cdot S(\vec{v})$ como sendo o $k$-vetor de símbolos em $\Sigma_{\sqcup}$

$$
\vec{b} \cdot S(\vec{v})=\left[\sigma_{1}, \ldots, \sigma_{k}\right]
$$

onde cada $\sigma_{i}=s_{i}\left(v_{i}\right)$ se $b_{i}=1$, e é $\longleftarrow$ se $b_{i}=0$.

Se $A$ é um alinhamento e $\vec{v}=[j, j, \ldots, j]$, então $A(\vec{v})$, também denotada por $A(j)$, é a $j$-ésima coluna do alinhamento $A$, e $A\left(j_{1} \ldots j_{2}\right)$ são as colunas $j_{1}, j_{1}+1, \ldots, j_{2}$ de $A$.

\subsubsection{Pontuação-SP}

Genericamente, a pontuação-SP de um $k$-vetor $\vec{\sigma}=\left[\sigma_{1}, \sigma_{2}, \ldots, \sigma_{k}\right]$ em $\Sigma_{\sqcup}$ é definida como

$$
v \mathrm{SP}_{\gamma}(\vec{\sigma})=\sum_{h=1}^{k-1} \sum_{i=h+1}^{k} \gamma_{\sigma_{h} \rightarrow \sigma_{i}} .
$$

Logo, a pontuação-SP de um alinhamento $A$ é a soma das pontuações-SP de suas colunas, ou seja,

$$
v \mathrm{SP}_{\gamma}(A)=\sum_{j=1}^{|A|} v \mathrm{SP}_{\gamma}(A(j)) .
$$

Lembramos que, para uma $k$-upla de sequências $S$, a função optSP ${ }_{\gamma}$ é tal que

$$
\operatorname{optSP}_{\gamma}(S)=\min _{A \in \mathcal{A}_{S}}\left\{v \mathrm{SP}_{\gamma}(A)\right\}
$$

e que dizemos que um alinhamento $A$ de $S$ é $v \operatorname{SP}_{\gamma^{-}}$ótimo se optSP $\operatorname{SP}_{\gamma}(S)=v \operatorname{SP}_{\gamma}(A)$.

Como vimos no Capítulo 2, o Problema AVS é: para uma matriz de pontuação fixa, dada uma $k$-upla $S$, determinar $\operatorname{optSP}_{\gamma}(S)$. Para resolver o problema, computamos uma tabela $D$ indexada por elementos em $\vec{V}_{S}$ que armazena as pontuações-SP dos alinhamentos $v \mathrm{SP}_{\gamma}$-ótimos dos prefixos de $S$. Mais precisamente, para cada $\vec{v} \in \vec{V}_{S}, D(\vec{v})=\operatorname{opt} \mathrm{SP}_{\gamma}(S(\vec{v}))$ e pode ser computada assim:

$$
D(\vec{v})= \begin{cases}0 & \text { se } \vec{v}=\mathbf{0}^{k}, \\ \min _{\vec{b} \in \mathcal{B}_{k}, \vec{b} \leq \vec{v}}\left\{D(\vec{v}-\vec{b})+v \mathrm{SP}_{\gamma}(\vec{b} \cdot S(\vec{v}))\right\} & \text { caso contrário. }\end{cases}
$$


Vamos verificar por indução em $\vec{v}$ que de fato $D(\vec{v})=o p t \mathrm{SP}_{\gamma}(S(\vec{v}))$. Note primeiramente que vale para $\vec{v}=\mathbf{0}^{k}$, pois $D\left(\mathbf{0}^{k}\right)=0=\operatorname{optSP} \gamma(\epsilon, \epsilon, \ldots, \epsilon)$. Para $\vec{v}>\mathbf{0}^{k}\left(\mathbf{0}^{k}<\vec{v}\right)$, vamos mostrar a seguir primeiro que

$$
\operatorname{optSP}(S(\vec{j})) \geq D(\vec{v})=\min _{\vec{b} \in \mathcal{B}_{k}, \vec{b} \leq \vec{v}}\left\{D(\vec{v}-\vec{b})+v \mathrm{SP}_{\gamma}(\vec{b} \cdot S(\vec{v}))\right\}
$$

e depois que

$$
D(\vec{v})=\min _{\vec{b} \in \mathcal{B}_{k}, \vec{b} \leq \vec{v}}\left\{D(\vec{v}-\vec{b})+v \mathrm{SP}_{\gamma}(\vec{b} \cdot S(\vec{v}))\right\} \geq \operatorname{optSP}_{\gamma}(S(\vec{j}))
$$

Suponha que $A$ é um alinhamento $v \mathrm{SP}_{\gamma}$-ótimo de $S(\vec{v})$ e $\vec{b}$ é um vetor tal que $A(|A|)=$ $\vec{b} \cdot S(\vec{v})$, ou seja, $\vec{b} \cdot S(\vec{v})$ é a última coluna de $A$. Seja $B$ um alinhamento $v \mathrm{SP}_{\gamma}$-ótimo de $S(\vec{v}-\vec{b})$. Por hipótese de indução optSP $\gamma(S(\vec{v}-\vec{b}))=D(\vec{v}-\vec{b})$. Segue daí que

$$
\begin{aligned}
\operatorname{optSP}_{\gamma}(S(\vec{v})) & =v \mathrm{SP}_{\gamma}(A)=v \mathrm{SP}_{\gamma}\left(A(1 \ldots|A|-1)+v \mathrm{SP}_{\gamma}(\vec{b} \cdot S(\vec{v}))\right. \\
& \geq v \mathrm{SP}_{\gamma}(B)+v \mathrm{SP}_{\gamma}(\vec{b} \cdot S(\vec{v}))=\operatorname{optSP} \gamma(S(\vec{v}-\vec{b}))+v \mathrm{SP}_{\gamma}(\vec{b} \cdot S(\vec{v})) \\
& =D(\vec{v}-\vec{b})+v \mathrm{SP}_{\gamma}(\vec{b} \cdot S(\vec{v})) \geq \min _{\vec{b} \in \mathcal{B}_{k}, \vec{b} \leq \vec{v}}\left\{D(\vec{v}-\vec{b})+v \operatorname{SP}_{\gamma}(\vec{b} \cdot S(\vec{v}))\right\} \\
& =D(\vec{v})
\end{aligned}
$$

e, portanto, vale (6.4).

Suponha agora que $\vec{b}$ minimiza $D(\vec{v}-\vec{b})+v \mathrm{SP}_{\gamma}(\vec{b} \cdot S(\vec{v})), B^{\prime}$ um alinhamento $v \mathrm{SP}_{\gamma}$-ótimo de $S(\vec{v}-\vec{b}), A^{\prime}$ um alinhamento de $S(\vec{v})$ de tal modo que $A^{\prime}\left(1 \ldots\left|A^{\prime}\right|-1\right)=B^{\prime}$ e $A^{\prime \prime}$ um alinhamento $v \mathrm{SP}_{\gamma}$-ótimo de $S(\vec{v})$. Por hipótese de indução, $D(\vec{v}-\vec{b})=\operatorname{optSP}_{\gamma}(S(\vec{v}-\vec{b}))$. Segue que

$$
\begin{aligned}
D(\vec{v}) & =\min _{\vec{b} \in \mathcal{B}_{k}, \vec{b} \leq \vec{v}}\left\{D(\vec{v}-\vec{b})+v \mathrm{SP}_{\gamma}(\vec{b} \cdot S(\vec{v}))\right\}=D(\vec{v}-\vec{b})+v \mathrm{SP}_{\gamma}(\vec{b} \cdot S(\vec{v})) \\
& =\operatorname{optSP}_{\gamma}(S(\vec{v}-\vec{b}))+v \mathrm{SP}_{\gamma}(\vec{b} \cdot S(\vec{v}))=v \mathrm{SP}_{\gamma}\left(B^{\prime}\right)+v \mathrm{SP}_{\gamma}(\vec{b} \cdot S(\vec{v}))=v \operatorname{SP}_{\gamma}\left(A^{\prime}\right) \\
& \geq v \operatorname{SP}_{\gamma}\left(A^{\prime \prime}\right)=o p t \operatorname{SP}_{\gamma}(S(\vec{v}))
\end{aligned}
$$

e, portanto, vale (6.5).

Como valem $(6.4)$ e $(6.5)$, temos que vale $D(\vec{v})=\operatorname{optSP}_{\gamma}(S(\vec{v}))$ para $\vec{v}>\mathbf{0}^{k}$. Usando programação dinâmica, a tabela $D$ pode ter suas entradas calculada em ordem lexicográfica. Com essa ordem, garantimos que nenhuma entrada $D(\vec{v})$ será calculada antes de $D(\vec{v}-\vec{b})$ para cada $\vec{b} \in \mathcal{B}_{k}, \vec{b} \leq \vec{v}$. O valor de $\operatorname{optSP}_{\gamma}(S)$ é então $D(\vec{n})$ (onde, lembramos da definição, $\left.\vec{n}=\left[n_{1}, \ldots, n_{k}\right]\right)$.

Considerando que todas as sequências possuem comprimento $n$, o espaço utilizado para calcular a tabela $D$ é $\Theta\left((n+1)^{k}\right)$. Para computar uma entrada de $D$, o algoritmo avalia no máximo $2^{k}-1$ outras entradas, onde cada avaliação gasta tempo $\Theta\left(k^{2}\right)$ no pior caso, o que implica que o tempo gasto para determinar cada entrada de $D$ é $O\left(2^{k} k^{2}\right)$. Logo, o tempo total gasto é $O\left(2^{k} k^{2} \cdot(n+1)^{k}\right)$. 


\subsubsection{Critério $\mathrm{V}_{\gamma}^{1}$}

$\mathrm{O}$ critério $\mathrm{V}_{\gamma}^{1}$ leva em conta o comprimento do alinhamento para a sua pontuação. A pontuação de um alinhamento $A$ conforme (6.1) é

$$
\mathrm{V}_{\gamma}^{1}(A)=\frac{v \mathrm{SP}_{\gamma}(A)}{|A|}
$$

se $|A|>0$. Um alinhamento $\mathrm{V}_{\gamma}^{1}$-ótimo de $S$, que é um alinhamento ótimo para esse critério, tem valor

$$
\operatorname{OPT}_{\gamma}^{1}(S)=\min _{A \in \mathcal{A}_{S}}\left\{\mathrm{~V}_{\gamma}^{1}(A)\right\}
$$

e o nosso problema é: para uma matriz $\gamma$ fixa, dada uma $k$-upla $S$, determinar $\operatorname{OPT}_{\gamma}^{1}(S)$.

\section{Algoritmo}

Uma idéia para a solução do problema é, em vez de diretamente computar o valor de um alinhamento $\mathrm{V}_{\gamma}^{1}$-ótimo de $S$, calcular a pontuação-SP mínima considerando cada possível comprimento de um alinhamento. Em outras palavras, computamos as entradas de uma tabela $D$ que é indexada por $\vec{V}_{S} \times\{0,1, \ldots, N\}$. A entrada $D(\vec{v}, L)$ armazena o valor de um alinhamento de $S(\vec{v})$ de comprimento $L$ de menor pontuação-SP. Note que $D\left(\mathbf{0}^{k}, 0\right)=0$. Além disso, $D\left(\vec{v} \neq \mathbf{0}^{k}, 0\right)$ não pode armazenar um valor de um alinhamento simplesmente porque não existe um de comprimento zero quando nem todas as sequências de $S$ têm comprimento zero. Similarmente, $D\left(\mathbf{0}^{k}, L \neq 0\right)$ não armazena um valor de qualquer alinhamento porque se todas as sequências de $S$ têm comprimento zero, então o único alinhamento de $S$ é um alinhamento de comprimento zero. Logo, as entradas da tabela podem ser calculadas pela seguinte relação de recorrência:

$$
D(\vec{v}, L)= \begin{cases}0 & \text { se } \vec{v}=\mathbf{0}^{k} \text { e } L=0, \\ \infty & \text { se } \vec{v}=\mathbf{0}^{k} \text { e } L \neq 0, \\ \infty & \text { se } \vec{v} \neq \mathbf{0}^{k} \text { e } L=0, \\ \min _{\vec{b} \in \mathcal{B}_{k}, \vec{b} \leq \vec{v}}\left\{D(\vec{v}-\vec{b}, L-1)+v \operatorname{SP}_{\gamma}(\vec{b} \cdot S(\vec{v}))\right\} & \text { caso contrário. }\end{cases}
$$

A tabela $D$ é computada para todos os valores possíveis para $L=0, \ldots, N$. Então, o valor

$$
\mathrm{OPT}_{\gamma}^{1}(S)=\min _{L}\left\{\frac{D(\vec{n}, L)}{L}\right\}
$$

é devolvido. O Algoritmo 5 detalha o procedimento descrito.

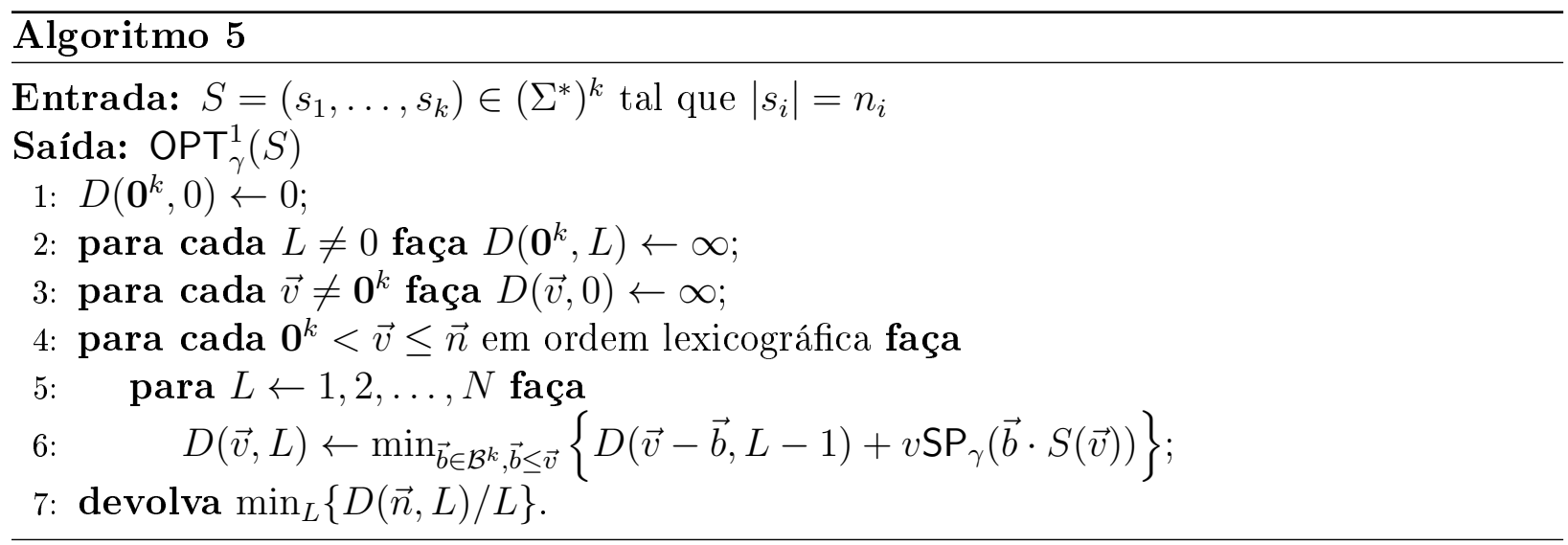


Para a análise do tempo e espaço gastos pelo Algoritmo 5, consideramos que todas as sequências possuem comprimento $n$, o que implica que o espaço utilizado para calcular a tabela de programação dinâmica é $\Theta\left(N(n+1)^{k}\right)$. O tempo gasto pelo algoritmo é o tempo para preencher a tabela $D$ mais o tempo gasto para a execução da Linha 7 do Algoritmo 5. Para calcular cada entrada de $D$ na Linha 6, o algoritmo gasta tempo $O\left(2^{k} k^{2}\right)$ e, portanto, desde que a tabela $D$ possui $\Theta\left(N(n+1)^{k}\right)$ entradas, o algoritmo consome tempo $O\left(2^{k} k^{2} \cdot N(n+1)^{k}\right)$ para computar a tabela $D$. A Linha 7 gasta tempo $\Theta(N)$. Portanto, o consumo total de tempo é

$$
O\left(2^{k} k^{2} \cdot N(n+1)^{k}\right)+\Theta(N)=O\left(2^{k} k^{2} \cdot N(n+1)^{k}\right) .
$$

Desde que $N=k n$, segue que o tempo gasto pelo algoritmo é $O\left(2^{k} k^{3}(n+1)^{k+1}\right)$.

\subsubsection{Critério $\mathrm{V}_{\gamma}^{2}$}

Dado um alinhamento $A$ de $S$, a pontuação do alinhamento $A$ segundo (6.2) é

$$
\mathrm{V}_{\gamma}^{2}(A)=\sum_{h=1}^{k-1} \sum_{i=h+1}^{k} v \mathrm{~N}_{\gamma}\left(A_{\{h, i\}}\right)
$$

e um alinhamento $\mathrm{V}_{\gamma}^{2}$-ótimo é aquele de pontuação

$$
\mathrm{OPT}_{\gamma}^{2}(S)=\min _{A \in \mathcal{A}_{S}}\left\{\mathrm{~V}_{\gamma}^{2}(A)\right\}
$$

Assim, nosso problema é: para uma matriz fixa $\gamma$, dada uma $k$-upla $S$, determinar $\mathrm{OPT}_{\gamma}^{2}(S)$.

Seja um $\left(\begin{array}{c}k \\ 2\end{array}\right)$-vetor de números naturais $\vec{L}$ indexado por conjuntos de dois números inteiros $\{h, i\}$ tais que $1 \leq h<i \leq k$. Denotamos por $L_{h i}$ o elemento de $\vec{L}$ de índice $\{h, i\}$. Então,

$$
\vec{L}=\left[L_{12}, L_{13}, \ldots, L_{1 k}, L_{23}, \ldots L_{2 k}, \ldots, L_{(k-1) k}\right]
$$

Com essa notação podemos representar por um vetor $\vec{L}$ os comprimentos dos alinhamentos induzidos de um alinhamento. Se $A$ é um alinhamento e $\left|A_{\{h, i\}}\right|=L_{h i}$ para cada $h, i$, dizemos que $\vec{L}$ é o comprimento induzido de $A$. Para uma $k$-upla $S=\left(s_{1}, \ldots, s_{k}\right)$, onde $\left|s_{i}\right|=n_{i}$ para cada $i$ definimos

$$
\mathbb{L}=\left\{\vec{L}=\left[L_{12}, L_{13}, \ldots, L_{1 k}, L_{23}, \ldots L_{2 k}, \ldots, L_{(k-1) k}\right]: 0 \leq L_{h i} \leq n_{h}+n_{i}\right\} .
$$

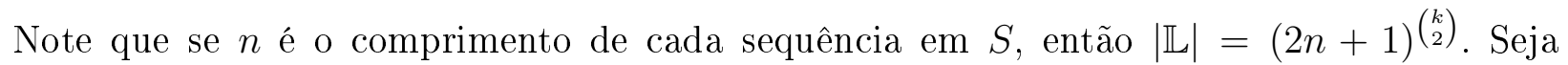
$\vec{b}=\left[b_{1}, \ldots, b_{k}\right]$ um $k$-vetor binário. Sobrecarregando o operador "-", definimos $\vec{L}-\vec{b}$ como sendo um $\left(\begin{array}{l}k \\ 2\end{array}\right)$-vetor de inteiros $\overrightarrow{L^{\prime}}$ que é obtido a partir de $\vec{L}$ e de $\vec{b}$ tal que, para cada $h, i$,

$$
L_{h i}^{\prime}= \begin{cases}L_{h i} & \text { se } b_{h}=b_{i}=0 \\ L_{h i}-1 & \text { caso contrário. }\end{cases}
$$

Claramente, se $\overrightarrow{L^{\prime}} \geq 0$, então $L^{\prime} \in \mathbb{L}$. É importante notar que se $\vec{L}$ é o comprimento induzido de um alinhamento $A$ de $S(\vec{v})$ e $\vec{b}$ é um $k$-vetor binário tal que $\vec{b} \cdot S(\vec{v})$ é a última coluna de $A$, então $\overrightarrow{L^{\prime}}=\vec{L}-\vec{b}$ é o comprimento induzido do alinhamento $A(1 \ldots|A|-1)$.

Seja $\vec{\gamma}$ um $\left(\begin{array}{l}k \\ 2\end{array}\right)$-vetor de matrizes de pontuação indexado por um conjunto de dois números inteiros $\{h, i\}$ tais que $1 \leq h<i \leq k$. Denotamos por $\gamma^{(h i)}$ o elemento de $\vec{\gamma}$ de índice $\{h, i\}$. 
Então,

$$
\vec{\gamma}=\left[\gamma^{(12)}, \gamma^{(13)}, \ldots, \gamma^{(1 k)}, \gamma^{(23)}, \ldots, \gamma^{(2 k)}, \ldots, \gamma^{((k-1) k)}\right]
$$

Definimos a pontuação $\vec{\gamma}-\mathrm{SP}$ de $A$ por

$$
v \mathrm{SP}_{\vec{\gamma}}(A)=\sum_{h=1}^{k-1} \sum_{i=h+1}^{k} v \mathrm{~A}_{\gamma(h i)}\left(A_{\{h, i\}}\right) .
$$

Definindo a pontuação $\vec{\gamma}$-SP de um $k$-vetor $\vec{\sigma}=\left[\sigma_{1}, \ldots, \sigma_{k}\right]$ em $\Sigma_{\longleftarrow}$ como

$$
v \mathrm{SP}_{\vec{\gamma}}(\vec{\sigma})=\sum_{h=1}^{k-1} \sum_{i=h+1}^{k} \gamma_{\sigma_{h} \rightarrow \sigma_{i}}^{(h i)}
$$

podemos, alternativamente, calcular a pontuação $\vec{\gamma}$-SP do alinhamento $A$ por

$$
v \mathrm{SP}_{\vec{\gamma}}(A)=\sum_{j} v \mathrm{SP}_{\vec{\gamma}}(A(j))
$$

\section{Algoritmo}

Antes de mostrar um algoritmo para o caso geral para encontrar $\mathrm{OPT}_{\gamma}^{2}(S)$, descrevemos primeiramente um algoritmo para o caso especial $k=3$, ou seja, quando $S=\left(s_{1}, s_{2}, s_{3}\right)$. Nessa versão, suponha que um oráculo informa previamente qual é o comprimento induzido $\overrightarrow{\mathrm{L}}=\left[\mathrm{L}_{12}, \mathrm{~L}_{13}, \mathrm{~L}_{23}\right]$ de um alinhamento $\mathrm{V}_{\gamma}^{2}$-ótimo de $S$. Isto quer dizer que, para algum alinhamento $\mathrm{V}_{\gamma}^{2}$-ótimo $A$ de $S$, temos que $\mathrm{L}_{h i}=\left|A_{\{h, i\}}\right|$ para cada $h, i$.

Observe que conhecer os valores de $\mathrm{L}_{12}, \mathrm{~L}_{13}$ e $\mathrm{L}_{23}$ não implica, usando somente essa informação, em conhecer o alinhamento $\mathrm{V}_{\gamma}^{2}$-ótimo $A$. Em geral, nem mesmo podemos inferir qual é o valor de $|A|$. Por exemplo,

$$
\left[\begin{array}{ccc}
s_{1}(1) & s_{1}(2) & \sqcup \\
s_{2}(1) & \sqcup & s_{2}(2) \\
\sqcup & s_{3}(1) & s_{3}(2)
\end{array}\right] \quad \text { e } \quad\left[\begin{array}{cccc}
s_{1}(1) & s_{1}(2) & \sqcup & \sqcup \\
s_{2}(1) & \sqcup & s_{2}(2) & \sqcup \\
s_{3}(1) & \sqcup & \sqcup & s_{3}(2)
\end{array}\right]
$$

são alinhamentos de comprimentos diferentes mas de mesmo comprimento induzido para $\left(s_{i}, s_{2}, s_{3}\right)$ onde $\left|s_{1}\right|=\left|s_{2}\right|=\left|s_{3}\right|=2$, e $\mathrm{L}_{12}=\mathrm{L}_{13}=\mathrm{L}_{23}=3$.

Entretanto, com o conhecimento de $\vec{L}=\left[\mathrm{L}_{12}, \mathrm{~L}_{13}, \mathrm{~L}_{23}\right]$, segue que

$$
\begin{aligned}
\mathrm{OPT}_{\gamma}^{2}(S) & =\min _{A \in \mathcal{A}_{S}: \forall h, i\left(\mathrm{~L}_{h i}=\left|A_{\{h, i\}}\right|\right)}\left\{\sum_{h=1}^{k-1} \sum_{i=h+1}^{k} \frac{v \mathrm{~A}_{\gamma}\left(A_{\{h, i\}}\right)}{\mathrm{L}_{h i}}\right\} \\
& =\min _{A \in \mathcal{A}_{S}: \forall h, i\left(\mathrm{~L}_{h i}=\left|A_{\{h, i\}}\right|\right)}\left\{\sum_{h=1}^{k-1} \sum_{i=h+1}^{k} v \mathrm{~A}_{\gamma(h i)}\left(A_{\{h, i\}}\right)\right\},
\end{aligned}
$$

onde $\gamma^{(h i)}$ é uma matriz de pontuação obtida multiplicando os elementos de $\gamma$ por $1 / L_{h i}$. Então, para $\vec{L}$ fixo (porque o oráculo já garantiu ser o comprimento induzido de um alinhamento $\mathrm{V}_{\gamma}^{2}$-ótimo), computamos $\vec{\gamma}$ a fim de calcular as entradas de uma tabela $D_{\overrightarrow{\mathrm{L}}}$, onde

$$
D_{\overrightarrow{\mathrm{L}}}\left(\vec{v}=\left[v_{1}, v_{2}, v_{3}\right], \vec{L}=\left[L_{12}, L_{13}, L_{23}\right]\right)=\min _{A \in \mathcal{A}_{S(\overrightarrow{1} \ldots \vec{v}): \forall h, i\left(L_{h i}=\left|A_{\{h, i\}}\right|\right)}}\left\{\sum_{h<i} v \mathrm{~A}_{\gamma(h i)}\left(A_{\{h, i\}}\right)\right\}
$$


corresponde à pontuação $\vec{\gamma}$-SP de um alinhamento de menor pontuação $\vec{\gamma}-\mathrm{SP}$ quando o comprimento induzido é $\vec{L}$. A tabela $D_{\vec{L}}$ pode ser então calculada usando a recorrência

$$
D_{\overrightarrow{\mathrm{L}}}(\vec{v}, \vec{L})= \begin{cases}0 & \text { se } \vec{v}=\mathbf{0}^{k} \text { e } \vec{L}=\mathbf{0}^{\left(\begin{array}{c}
k \\
2
\end{array}\right),} \\
\infty & \text { se } \left.\vec{v}=\mathbf{0}^{k} \text { e } \vec{L} \neq \mathbf{0}^{(} \begin{array}{c}
k \\
2
\end{array}\right), \\
\infty & \text { se } \left.\vec{v} \neq \mathbf{0}^{k} \text { e } \vec{L}=\mathbf{0}^{(k} \begin{array}{c}
k \\
2
\end{array}\right), \\
\min _{\vec{b} \in \mathcal{B}_{k}, \vec{b} \leq \vec{v}, \vec{b} \leq \vec{L}} D_{\overrightarrow{\mathrm{L}}}(\vec{v}-\vec{b}, \vec{L}-\vec{b})+v \mathrm{SP}_{\vec{\gamma}}(\vec{b} \cdot S(\vec{v})) & \text { caso contrário, }\end{cases}
$$

onde $\vec{b} \leq \vec{L}$ também é uma sobrecarga que significa que $\vec{L}-\vec{b} \geq \mathbf{0}^{\left(\begin{array}{c}k \\ 2\end{array}\right)}$.

Neste caso, se $\overrightarrow{\mathrm{L}}$ é o comprimento induzido de um alinhamento $\mathrm{V}_{\gamma}^{2}$-ótimo de $S$, então $\mathrm{OPT}_{\gamma}^{2}(S)=D_{\overrightarrow{\mathrm{L}}}(\vec{n}, \overrightarrow{\mathrm{L}})$. Se cada sequência tem comprimento $n$, então, o espaço total gasto

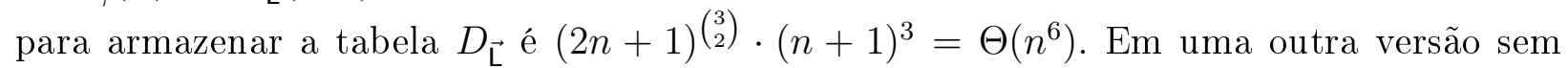
oráculo, ou seja, quando $\vec{L}$ não é conhecido, a computação deve ser repetida para cada elemento $\vec{L} \in \mathbb{L}$ mas o espaço pode ser reutilizado não havendo a necessidade de espaço adicional.

Se $\vec{L}$ é conhecido, o tempo gasto pelo algoritmo é aquele usado para calcular todas as entradas de $D_{\overrightarrow{\mathrm{L}}}$. Como $D_{\overrightarrow{\mathrm{L}}}$ possui $\Theta\left(n^{6}\right)$ entradas e cada entrada consome tempo $O\left(2^{k} k^{2}\right)$ para ser computado, o tempo total gasto é $O\left(2^{k} k^{2} \cdot n^{6}\right)$. Na versão sem oráculo, ou seja, se $\overrightarrow{\mathrm{L}}$ não é conhecido, o tempo gasto por $\overrightarrow{\mathrm{L}}$ deve ser multiplicado pelo total de elementos em $\mathbb{L}$ que é $(2 n+1)^{3}$. Portanto, neste último caso, $O\left(2^{k} k^{2} \cdot(2 n+1)^{3} n^{6}\right)=O\left(2^{k} k^{2} \cdot n^{9}\right)$ é o tempo total gasto para todas as computações.

O algoritmo a seguir é uma extensão natural do algoritmo acima quando $k$ não é fixo e nem L é conhecido. Denotamos aqui, dada uma matriz de pontuação $\gamma$ e um comprimento induzido $\mathrm{L}$, o $\left(\begin{array}{l}k \\ 2\end{array}\right)$-vetor de matrizes de pontuação $\gamma \times \mathrm{L}=\left[\gamma^{(12)}, \ldots, \gamma^{k(k-1)}\right]$, onde, para cada $h<i$, temos que $\gamma^{(h i)}$ é obtida dividindo cada entrada de $\gamma$ por $L_{h i}$.

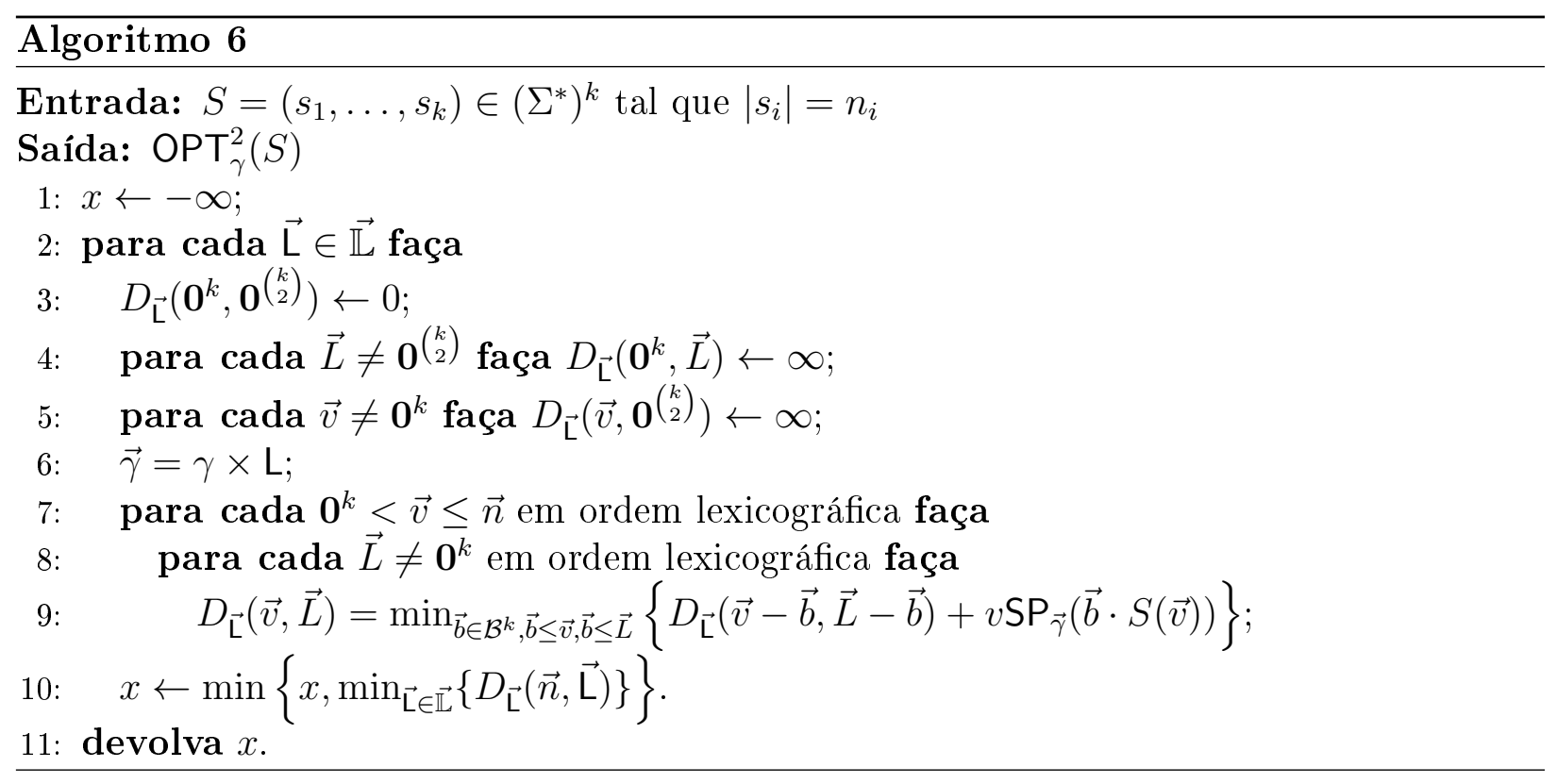

O Algoritmo 6, para $k$ sequências de comprimento $n$, gasta espaço $(2 n+1)^{\left(\begin{array}{l}k \\ 2\end{array}\right)} \cdot(n+1)^{k}$ para armazenar uma tabela $D_{\overrightarrow{\mathrm{L}}}$. Para cada um dos $(2 n+1)^{\left(\begin{array}{c}k \\ 2\end{array}\right)}$ valores $\overrightarrow{\mathrm{L}} \in \overrightarrow{\mathbb{L}}$ a tabela $D_{\overrightarrow{\mathrm{L}}}$ é recalculada. Como cada entrada gasta tempo $O\left(2^{k} k^{2}\right)$ para ser computada, o tempo total 
gasto é

$$
O\left(2^{k} k^{2} \cdot(2 n+1)^{\left(\begin{array}{c}
k \\
2
\end{array}\right)} \cdot(2 n+1)^{\left(\begin{array}{c}
k \\
2
\end{array}\right)}(n+1)^{k}=\left(1+\frac{1}{2 n+1}\right)^{k}(2 n+1)^{k^{2}} k^{2}\right)
$$

que é $\left.O\left((2 n+1)^{k^{2}} k^{2}\right)\right)$ se $k \leq 2 n+1$ pois, sendo $(1+1 / k)^{k} \leq e=2,718281828 \ldots$ onde $e$ é a constante de Euler, temos que $(1+1 /(2 n+1))^{k} \leq(1+1 / k)^{k} \leq e$ também é constante.

\section{O problema de existência de um alinhamento $A$ para um dado $\vec{L}$}

Para muitos valores $\vec{L} \in \mathbb{L}$ não existe um alinhamento correspondente. Em vez de examinar $\Theta\left(2^{k}\right)$ entradas no pior caso para constatar esse fato, queremos detectar rapidamente se existe um alinhamento correspondente para $\vec{L}$. Esse problema pode ser assim enunciado.

Problema 7. RACI: Reconhecimento de um alinhamento pelo comprimento induzido

Dado um $k$-vetor $\vec{n}$ de inteiros, correspondente aos comprimentos das sequências em $S=\left(s_{1}, \ldots, s_{k}\right)$ e um $\left(\begin{array}{c}k \\ 2\end{array}\right)$-vetor de números inteiros $\vec{L}$ decidir se existe um alinhamento $A$ de $S$ tal que $\vec{L}$ é comprimento induzido de $A$.

Note que um alinhamento de $(s, t)$ possui comprimento $L$ se e somente se a quantidade de substituições (de símbolos iguais ou não) nesse alinhamento é exatamente $|s|+|t|-L$. Portanto, o problema acima pode ser facilmente reformulado trocando $\vec{L}$ por um $\left(\begin{array}{l}k \\ 2\end{array}\right)$-vetor $\vec{X}$ de mesmo tipo de $\vec{L}$, onde $X_{h i}=\left|s_{h}\right|+\left|s_{i}\right|-L_{h i}=n_{h}+n_{i}-L_{h i}$ para cada $h$ e cada $i$. Assim, o problema pode ser reescrito para o problema de, dados $\vec{n}$ e $\vec{X}$, decidir se existe um alinhamento $A$ de algum $S=\left(s_{1}, \ldots, s_{k}\right)$, onde $\left|s_{i}\right|=n_{i}$ para cada $i$ e tal que cada $A_{\{i, j\}}$ possui $X_{i j}$ substituições. Exceto pela formulação diferente, esse é exatamente o problema conhecido como Reconhecimento de padrão de intersecção( Recognizing intersection patterns) que enunciamos a seguir usando uma matriz de números naturais $M$ com $k$ linhas e $k$ colunas no lugar dos vetores $\vec{n}$ e $\vec{X}$ : para cada $i, M[i, i]=n_{i}$ e, para cada par de inteiros $h, i, M[h, i]=X_{h, i}$.

Problema 8. RPI: Reconhecimento de padrão de intersecção

Dada uma matriz de inteiros $M$ com $k$ linhas e $k$ colunas, decidir se existe uma coleção de conjuntos $c_{1}, \ldots, c_{k}$ tais que $\left|c_{h} \cap c_{i}\right|=M[h, i]$.

Como exemplo da equivalência dos problemas acima, suponha que $\vec{n}=[5,5,5]$ e que $X_{12}=X_{13}=2$ e $X_{23}=0$. A resposta ao nosso problema é Sim pois, para $\left(s_{1}, s_{2}, s_{3}\right)$, os alinhamentos induzidos do seguinte alinhamento com 11 colunas

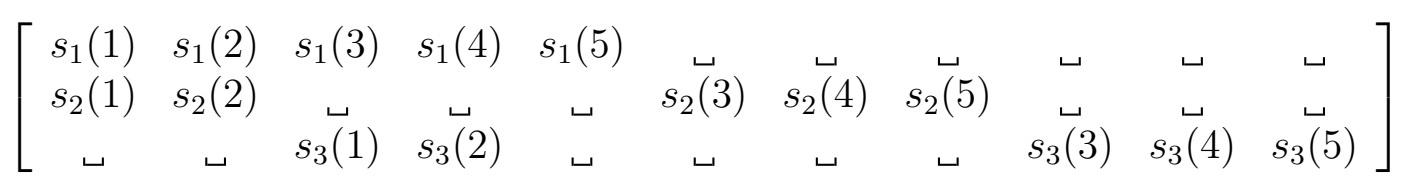

possuem os valores dados em $\vec{X}$. O Problema RPI para esta instância quer encontrar uma coleção de 3 conjuntos para a matriz

$$
M=\begin{array}{l|lll} 
& 1 & 2 & 3 \\
\hline 1 & 5 & 2 & 2 \\
2 & 2 & 5 & 0 \\
3 & 2 & 0 & 5
\end{array}
$$

e a resposta é Sim como vemos na figura a seguir. 


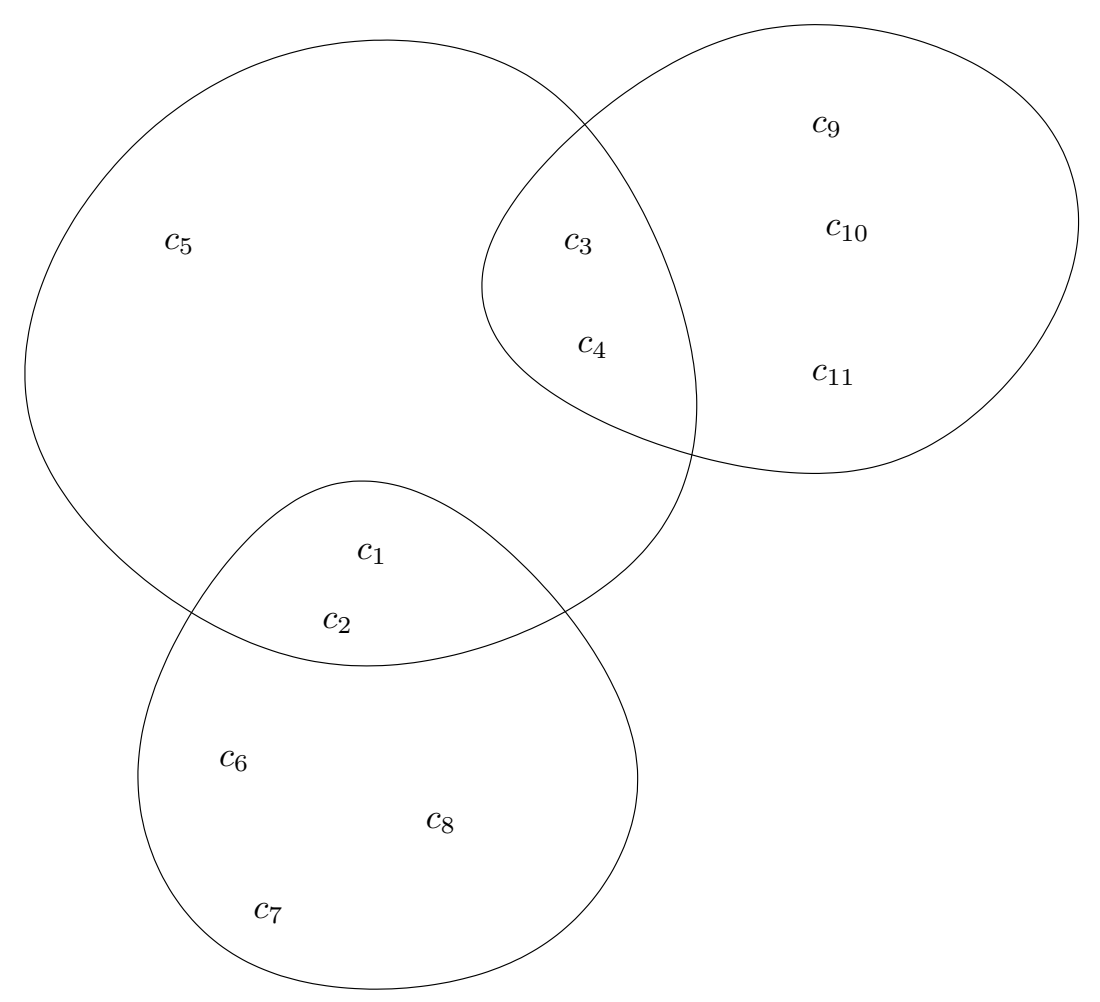

Em um outro exemplo, suponha que $\vec{n}=[5,5,5], X_{12}=X_{13}=3$ e $X_{23}=0$. Nesse caso a resposta é Não. Para verificar isto, suponha por contradição que existe um alinhamento $A$ de uma 3-upla $S=\left(s_{1}, s_{2}, s_{3}\right)$ para essa entrada. Como $X_{12}=3$, temos que $s_{1}$ e $s_{2}$ devem ter 3 caracteres alinhados que não podem estar alinhados com $s_{3}$ porque $X_{23}=0 \mathrm{e}$, de forma análoga, como $X_{13}=3$, temos que $s_{1}$ e $s_{3}$ devem ter 3 caracteres alinhados que não podem estar alinhados com $s_{2}$. Logo, $s_{1}$ deve ter pelo menos 6 símbolos o que é uma contradição.

Chvátal [Chv80] mostrou que para uma classe especial de matrizes $M$ em que $M[i, i]=3$ para cada $i$, o Problema RPI é NP-completo. Portanto, o Problema 7 é NP-completo quando a quantidade de símbolos em cada sequência é exatamente 3 e é difícil se as sequências possuem comprimento arbitrário.

\subsubsection{Critério $\mathrm{V}_{\gamma}^{3}$}

Dado um alinhamento $A$ de $S$, conforme (6.3), temos

$$
\mathrm{V}_{\gamma}^{3}(A)=\frac{v \mathrm{SP}_{\gamma}(A)}{\sum_{h=1}^{k-1} \sum_{i=h+1}^{k}\left|A_{\{h, i\}}\right|}
$$

se $|A|>0$. Lembre que um alinhamento $\mathrm{V}_{\gamma}^{3}$-ótimo é aquele de pontuação

$$
\operatorname{OPT}_{\gamma}^{3}(S)=\min _{A \in \mathcal{A}_{S}}\left\{\mathrm{~V}_{\gamma}^{3}(A)\right\}
$$

e que o seguinte problema está a ele associado: para uma matriz de pontuação $\gamma$ fixa, dada uma $k$-upla $S$ de sequências, determinar $\operatorname{OPT}_{\gamma}^{3}(S)$.

\section{Algoritmo}

Assim como os outros critérios, descrevemos um algoritmo que usa programação dinâmica para a solução do problema. Aqui, cada entrada $D(\vec{v}, L)$ da tabela armazena a pontuação-SP de um alinhamento $A$ do prefixo $S(\vec{v})$ de menor pontuação SP tal que $\sum_{i<h}\left|A_{\{i, h\}}\right|=L$. 
Os vetores booleanos $\vec{b}$ são utilizados para representar a contribuição na soma dos comprimentos dos alinhamentos induzidos. Assim, definimos $\|\vec{b}\|=\left(\begin{array}{l}k \\ 2\end{array}\right)-\sum_{h<i, b_{h}=b_{i}=0} 1$. Note que se $\vec{v}-\vec{b}$ é a última coluna de uma alinhamento $A$ e $L=\sum_{h=1}^{k-1} \sum_{i=h+1}^{k}\left|A_{\{h, i\}}\right|$ é a soma dos comprimentos dos alinhamentos induzidos por $A$, então a soma dos comprimentos dos alinhamentos induzidos por $A(1 \ldots|A|-1)$ é $L-\|\vec{b}\|$. Logo,

$$
D(\vec{v}, L)= \begin{cases}0 & \text { se } \vec{v}=\mathbf{0}^{k} \text { e } L=0 \\ \infty & \text { se } \vec{v}=\mathbf{0}^{k} \text { e } L \neq 0 \\ \infty & \text { se } \vec{v} \neq \mathbf{0}^{k} \text { e } L=0 \\ \min _{\vec{b} \in \mathcal{B}_{k}, \vec{b} \leq \vec{v},\|\vec{b}\| \leq L}\left\{D(\vec{v}-\vec{b}, L-\|\vec{b}\|)+v \operatorname{SP}_{\gamma}(\vec{b} \cdot S(\vec{v}))\right\} & \text { caso contrário. }\end{cases}
$$

O Algoritmo 7 descreve a computação para encontrar o valor de $\operatorname{OPT}_{\gamma}^{3}(S)$ conforme discutido acima.

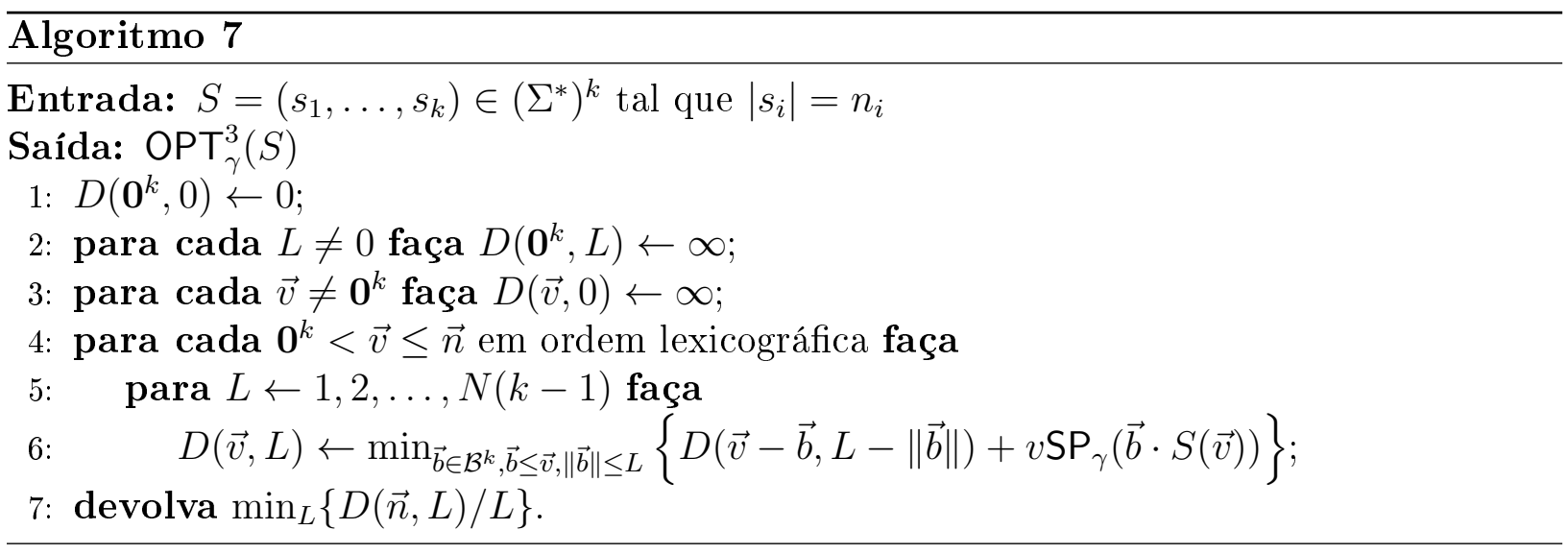

Considere que todas as sequências possuem comprimento $n$. A tabela $D$ é computada para todos os valores possíveis para $L=1, \ldots,\left(\begin{array}{c}k \\ 2\end{array}\right)(2 n)=n k^{2}-n k$ e, depois de calculada, determinamos

$$
o p t \mathrm{SP}_{\gamma}(S)=\min _{L}\left\{\frac{D(\vec{n}, L)}{L}\right\}
$$

Assim, o espaço utilizado para a tabela $D$ é $\left(n k^{2}-n k+1\right) \cdot(n+1)^{k}=\Theta\left(k^{2}(n+1)^{k+1}\right)$ e, desde que o tempo gasto para determinar cada entrada de $D$ é $O\left(2^{k} k^{2}\right)$, o tempo total gasto é $O\left(2^{k} k^{4}(n+1)^{k+1}\right)$.

\subsection{Complexidade}

Nesta seção estudamos a complexidade do problema do alinhamento de várias sequências para cada um dos novos critérios definidos neste texto. Para isto definimos o seguinte problema parametrizado.

Problema 9. AVSN- $\eta$ : Alinhamento de várias sequências com critério de pontuação $\mathrm{V}_{\gamma}^{\eta}$

Dados uma $k$-upla $S=\left(s_{1}, \ldots, s_{k}\right)$ de sequências em $\Sigma^{*}$ e um número racional $C$, decidir se, para uma matriz de pontuação fixa $\gamma$ com entradas inteiras e $\eta$ fixo, existe um alinhamento $A$ de $S$, tal que $\bigvee_{\gamma}^{\eta}(A) \leq C$.

Vamos provar que, para cada $\eta$, o Problema AVSN- $\eta$ é NP-completo para várias matrizes de pontuação. Note primeiramente que, dado um alinhamento $A$ e um racional $C$, podemos verificar em tempo polinomial que $\mathrm{V}_{\gamma}^{\eta}(A) \leq C$ o que implica que AVSN- $\eta$ está em NP. 
Para completar a prova, basta mostrarmos uma redução polinomial do Problema AVSd ao Problema AVSN- $\eta$. Conforme estudamos no Capítulo 2, AVSd é a versão de decisão do problema de alinhamento de várias sequências com o critério de pontuação-SP: dados uma $k$-upla $S=\left(s_{1}, \ldots, s_{k}\right)$ de sequências em $\Sigma^{*}$ e um número inteiro $C$, decidir se, para uma matriz de pontuação fixa $\gamma$ com entradas inteiras, existe um alinhamento $A$ de $S$, tal que $v \mathrm{SP}_{\gamma}(A) \leq C$. Conforme estudado naquele capítulo, o Problema AVSd é NP-completo considerando diferentes matrizes de pontuação.

Considere um alfabeto $\Sigma$ e uma matriz de pontuação $\gamma$ com entradas inteiras, para os quais o Problema AVSd é NP-completo. As restrições adicionais para $\gamma$ são $\gamma_{a \rightarrow b}=\gamma_{b \rightarrow a}$ e $\gamma_{\mathrm{a} \rightarrow \mathrm{b}}=0$ se e somente se $\mathrm{a}=\mathrm{b}$ para cada $\mathrm{a}, \mathrm{b} \in \Sigma_{\mathrm{\omega}}$. Considere um novo símbolo $\sigma \notin \Sigma_{\mathrm{v}} \mathrm{e}$ um alfabeto $\Sigma^{\sigma}=\Sigma \cup\{\sigma\}$. Seja $G$ um inteiro positivo de valor maior ou igual a cada entrada de $\gamma$. Definimos a matriz de pontuação $\gamma^{\sigma}$ tal que $\gamma_{\mathrm{a} \rightarrow \mathrm{b}}^{\sigma}=\gamma_{\mathrm{a} \rightarrow \mathrm{b}}, \gamma_{\mathrm{a} \rightarrow \sigma}^{\sigma}=\gamma_{\sigma \rightarrow \mathrm{a}}^{\sigma}=G$ e $\gamma_{\sigma \rightarrow \sigma}^{\sigma}=0$ para cada a, $\mathrm{b} \in \Sigma_{\sqcup}$. Vamos mostrar que, para $\Sigma^{\sigma}$ e $\gamma^{\sigma}$ fixos, o Problema AVSN- $\eta$ é NP-completo.

Considerando um alfabeto $\Sigma$ e uma matriz de pontuação $\gamma$ fixos, para uma instância $\left(S=\left(s_{1}, \ldots, s_{k}\right), C\right)$ do Problema AVSd, ponha $S^{L}=\left(s_{1} \sigma^{L}, \ldots, s_{k} \sigma^{L}\right)$, onde $L=N k^{2} M G$ $\operatorname{com} M=\max _{i}\left\{\left|s_{i}\right|\right\}$ e $N=\sum_{i=1}^{k}\left|s_{i}\right|$.

Seja $A=\left[s_{1}^{\prime}, \ldots, s_{k}^{\prime}\right]$ um alinhamento de $S^{L}$. Dizemos que $\left|\left\{i: s_{i}^{\prime}(j)=\sigma\right\}\right|$ é a quantidade de $\sigma$ na coluna $j$ de $A$ e que $j$ é uma coluna $\sigma$ se $k$ é a quantidade de $\sigma$ na coluna $j$. Se $j$ não é coluna $\sigma$ de $A$, mas cada $j+1, j+2, \ldots,|A|$ são colunas $\sigma$, então dizemos que $A(j+1 \ldots|A|)$ é a cauda do alinhamento $A$ e que a coluna $j$ é a base da cauda, e que o comprimento da cauda de $A$ é $|A|-j$. Dizemos ainda que um alinhamento de $S^{L}$ é canônico se o comprimento de sua cauda é $L$.

Se $A=\left[s_{1}^{\prime}, \ldots, s_{k}^{\prime}\right]$ é um alinhamento de $S$, então denotamos por $A^{L}$ o alinhamento canônico $A^{L}=\left[s_{1}^{\prime} \sigma^{L}, \ldots, s_{k}^{\prime} \sigma^{L}\right]$ de $S^{L}$. O seguinte resultado mostra um limitante superior para a pontuação de um alinhamento canônico.

Fato 84. Seja $A$ um alinhamento de $S$. Então, $v \mathrm{SP}_{\gamma^{\sigma}}\left(A^{L}\right)=v \mathrm{SP}_{\gamma}(A) \leq k^{2} M G$.

Prova. Suponha que $A=\left[s_{1}^{\prime}, \ldots, s_{k}^{\prime}\right]$. Cada sequência em $S$ tem comprimento não superior a $M$ o que implica que cada alinhamento induzido por $A$ possui não mais do que $2 M$ colunas. Cada entrada de $\gamma$ é menor ou igual a $G$. Portanto, para cada $h, i, v \mathrm{~A}_{\gamma}\left(s_{h}^{\prime}, s_{i}^{\prime}\right)=$ $v \mathrm{~A}_{\gamma}\left(s_{h}^{\prime} \sigma^{L}, s_{i}^{\prime} \sigma^{L}\right) \leq 2 M G$, o que implica que

$$
\begin{aligned}
v \mathrm{SP}_{\gamma^{\sigma}}\left(A^{L}\right) & =v \mathrm{SP}_{\gamma}(A)=\sum_{h=1}^{k-1} \sum_{i=h+1}^{k} v \mathrm{~A}_{\gamma}\left(A_{\{h, i\}}\right) \\
& \leq \sum_{h=1}^{k-1} \sum_{i=h+1}^{k} 2 M G=\left(\begin{array}{l}
k \\
2
\end{array}\right) 2 M G=\frac{k(k-1)}{2} 2 M G \leq k^{2} M G .
\end{aligned}
$$

O seguinte resultado mostra a relação entre alinhamentos canônicos e alinhamento $\mathrm{V}_{\gamma^{\sigma^{-}}}^{\eta}$ ótimos.

Lema 85. Seja $S$ uma $k$-upla de sequências. Para qualquer $\eta$, existe um alinhamento canônico de $S^{L}$ que é $\mathrm{V}_{\gamma^{\sigma}}^{\eta}$-ótimo.

Prova. Suponha por contradição que todo alinhamento canônico de $S^{L}$ não é $\mathrm{V}_{\gamma^{\sigma}}^{\eta}$-ótimo. Seja $A=\left[s_{1}^{\prime}, \ldots, s_{k}^{\prime}\right]$ um alinhamento $\mathrm{V}_{\gamma^{\sigma}}^{\eta}$-ótimo de $S^{L}$ com comprimento de cauda máxima cuja quantidade de $\sigma$ na base da cauda é máxima. Note que por hipótese $A$ não é canônico. 
Seja $q$ o índice da base da cauda de $A$. Como $A$ não é canônico, a coluna $q$ contém

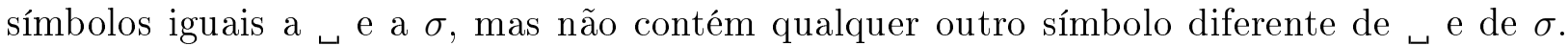
Seja $p$ o maior índice tal que $p<q$ e existe um inteiro $i$ onde $s_{i}^{\prime}(p)=\sigma$ e $s_{i}^{\prime}(q)=\sqcup$.

Seja $A^{\prime}=\left[s_{1}^{\prime \prime}, \ldots, s_{k}^{\prime \prime}\right]$ um alinhamento de $S^{L}$ igual ao alinhamento $A$ exceto pela colunas $p$ e $q$ que são alteradas conforme a regra a seguir. Para cada $h$, temos que

$$
s_{h}^{\prime \prime}= \begin{cases}s_{h}^{\prime} & \text { se } s_{h}^{\prime}(p) \neq \sigma \text { ou } s_{h}^{\prime}(q) \neq\llcorner, \\ s_{h}^{\prime}(1 \ldots p-1) \cdot \cdot^{\prime} \cdot s_{h}^{\prime}(p+1 \ldots q-1) \cdot \sigma \cdot s_{h}^{\prime}\left(q+1 \ldots\left|s_{h}^{\prime}\right|\right) & \text { caso contrário. }\end{cases}
$$

Note que o comprimento da cauda de $A^{\prime}$ é maior do que o de $A$, ou então $A$ e $A^{\prime}$ possuem caudas de mesmo comprimento mas a quantidade de $\sigma$ na base da cauda de $A^{\prime}$ é maior do que a em $A$. Logo, pela escolha de $A, A^{\prime}$ não é $\mathrm{V}_{\gamma^{\sigma}}^{\eta}$-ótimo.

Sejam $h, i$ inteiros. Classificamos o alinhamento induzido $A_{\{h, i\}}$ de $A$ da seguinte maneira:

1. tipo 1: quando $v \mathrm{~A}_{\gamma^{\sigma}}\left(A_{\{h, i\}}\right)=v \mathrm{~A}_{\gamma^{\sigma}}\left(A_{\{h, i\}}^{\prime}\right)$ e $\left|A_{\{h, i\}}\right|=\left|A_{\{h, i\}}^{\prime}\right|$;

2. tipo 2: quando $v \mathrm{~A}_{\gamma^{\sigma}}\left(A_{\{h, i\}}\right) \neq v \mathrm{~A}_{\gamma^{\sigma}}\left(A_{\{h, i\}}^{\prime}\right)$ e $\left|A_{\{h, i\}}\right|=\left|A_{\{h, i\}}^{\prime}\right|$. Nesse caso, a única possibilidade é que em uma das sequências, digamos $s_{h}^{\prime}$, temos $s_{h}^{\prime}(p)=\sigma$ e $s_{h}^{\prime}(q)={ }$, e na outra, a sequência $s_{i}^{\prime}$, temos $s_{i}^{\prime}(p)=x$ e $s_{i}^{\prime}(q)=\sigma$, onde $x \in \Sigma$. Por hipótese, $G \geq 0, G \geq \gamma_{x \rightarrow \sqcup}^{\sigma}$ e $G \geq \gamma^{\sigma} \rightarrow x$. Se $h<i$, segue que

$$
v \mathrm{~A}_{\gamma^{\sigma}}\left(A_{\{h, i\}}^{\prime}\right)=v \mathrm{~A}_{\gamma^{\sigma}}\left(A_{\{h, i\}}\right)-2 G+\gamma_{x \rightarrow \sqcup}^{\sigma} \leq v \mathrm{~A}_{\gamma^{\sigma}}\left(A_{\{h, i\}}\right),
$$

e se $h>i$, segue que

$$
v \mathrm{~A}_{\gamma^{\sigma}}\left(A_{\{h, i\}}^{\prime}\right)=v \mathrm{~A}_{\gamma^{\sigma}}\left(A_{\{h, i\}}\right)-2 G+\gamma^{\sigma} \rightarrow x \leq v \mathrm{~A}_{\gamma^{\sigma}}\left(A_{\{h, i\}}\right) .
$$

Portanto, $v \mathrm{~A}_{\gamma^{\sigma}}\left(A_{\{h, i\}}^{\prime}\right) \leq v \mathrm{~A}_{\gamma^{\sigma}}\left(A_{\{h, i\}}\right)$;

3. tipo 3: quando $\left|A_{\{h, i\}}\right| \neq\left|A_{\{h, i\}}^{\prime}\right|$. Nesse caso, a única possibilidade é que em uma das sequências, digamos $s_{h}^{\prime}$, temos que $s_{h}^{\prime}(p)=\sigma$ e $s_{h}^{\prime}(q)={ }_{\longleftarrow}$ e na outra, $s_{i}^{\prime}$, temos que $s_{i}^{\prime}(p)={ }_{\llcorner}$e $s_{i}^{\prime}(q)=\sigma$. Segue independentemente dos valores de $h, i$ que

$$
v \mathrm{~A}_{\gamma^{\sigma}}\left(A_{\{h, i\}}^{\prime}\right)=v \mathrm{~A}_{\gamma^{\sigma}}\left(A_{\{h, i\}}\right)-2 G \quad \text { e } \quad\left|A_{\{h, i\}}\right|=\left|A_{\{h, i\}}^{\prime}\right|+1 .
$$

Vamos mostrar a seguir que o lema vale para $\eta=1$. Como, para qualquer tipo e quaisquer inteiros $h, i$, vale $v \mathrm{~A}_{\gamma^{\sigma}}\left(A_{\{h, i\}}^{\prime}\right) \leq v \mathrm{~A}_{\gamma^{\sigma}}\left(A_{\{h, i\}}\right)$, temos que $v \operatorname{SP}_{\gamma^{\sigma}}\left(A^{\prime}\right) \leq v \operatorname{SP}_{\gamma^{\sigma}}(A)$. Suponha que $\left|A^{\prime}\right|=|A|$. Logo,

$$
\mathrm{V}_{\gamma^{\sigma}}^{1}\left(A^{\prime}\right)=\frac{v \mathrm{SP}_{\gamma^{\sigma}}\left(A^{\prime}\right)}{\left|A^{\prime}\right|} \leq \frac{v \mathrm{SP}_{\gamma^{\sigma}}(A)}{\left|A^{\prime}\right|}=\frac{v \mathrm{SP}_{\gamma^{\sigma}}(A)}{|A|}=\mathrm{V}_{\gamma^{\sigma}}^{1}(A)
$$

contrariando que $A^{\prime}$ não ser $\bigvee_{\gamma^{\sigma}}^{1}$-ótimo. Assumimos então que $\left|A^{\prime}\right| \neq|A|$. Logo, $\left|A^{\prime}\right|=$ $|A|-1$ e pelo menos um alinhamento $A_{\{h, i\}}$ é do tipo 3 , o que implica que $v \mathrm{~A}_{\gamma^{\sigma}}\left(A_{\{h, i\}}^{\prime}\right)=$ $v \mathrm{~A}_{\gamma^{\sigma}}\left(A_{\{h, i\}}\right)-2 G$. Segue que $v \mathrm{SP}_{\gamma^{\sigma}}\left(A^{\prime}\right) \leq v \mathrm{SP}_{\gamma^{\sigma}}(A)-2 G$. Logo,

$$
\mathrm{V}_{\gamma^{\sigma}}^{1}\left(A^{\prime}\right)=\frac{v \mathrm{SP}_{\gamma^{\sigma}}\left(A^{\prime}\right)}{\left|A^{\prime}\right|} \leq \frac{v \mathrm{SP}_{\gamma^{\sigma}}(A)-2 G}{|A|-1}
$$

Seja $B$ um alinhamento canônico. Pelo Fato 84 temos que $v \mathrm{SP}_{\gamma^{\sigma}}(B) \leq k^{2} M G$. Pela 
escolha de $A$, temos que $\mathrm{V}_{\gamma^{\sigma}}^{1}(A) \leq \mathrm{V}_{\gamma^{\sigma}}^{1}(B)$. Como $G \geq 1,|B| \geq L=N k^{2} M G$, segue que

$$
\mathrm{V}_{\gamma^{\sigma}}^{1}(A) \leq \mathrm{V}_{\gamma^{\sigma}}^{1}(B)=\frac{v \mathrm{SP}_{\gamma^{\sigma}}(B)}{|B|} \leq \frac{k^{2} M G}{N k^{2} M G}=\frac{1}{N} \leq G
$$

Sendo $v \operatorname{SP}_{\gamma^{\sigma}}(A) /|A| \leq G$ e $G \geq 1$, temos que

$$
\frac{v \mathrm{SP}_{\gamma^{\sigma}}(A)-2 G}{|A|-1} \leq \frac{v \mathrm{SP}_{\gamma^{\sigma}}(A)}{|A|}
$$

o que implica por (6.6) e pela definição de $\mathrm{V}_{\gamma^{\sigma}}^{1}(A)$ que

$$
\mathrm{V}_{\gamma^{\sigma}}^{1}\left(A^{\prime}\right) \leq \frac{v \mathrm{SP}_{\gamma^{\sigma}}(A)-2 G}{|A|-1} \leq \frac{v \mathrm{SP}_{\gamma^{\sigma}}(A)}{|A|}=\mathrm{V}_{\gamma^{\sigma}}^{1}(A)
$$

contrariando novamente $A^{\prime}$ não ser $\bigvee_{\gamma^{\sigma}}^{1}$-ótimo. Logo, existe um alinhamento canônico de $S^{L}$ que é $\mathrm{V}_{\gamma^{\sigma}}^{1}$-ótimo.

Mostramos agora que o lema vale para $\eta=2$. Se um alinhamento induzido $A_{\{h, i\}}$ é do tipo 1 ou 2, então $v \mathrm{~A}_{\gamma^{\sigma}}\left(A_{\{h, i\}}^{\prime}\right) \leq v \mathrm{~A}_{\gamma^{\sigma}}\left(A_{\{h, i\}}\right)$ e $\left|A_{\{h, i\}}^{\prime}\right|=\left|A_{\{h, i\}}\right|$ o que implica que

$$
v \mathrm{~N}_{\gamma^{\sigma}}\left(A_{\{h, i\}}^{\prime}\right)=\frac{v \mathrm{~A}_{\gamma^{\sigma}}\left(A_{\{h, i\}}^{\prime}\right)}{\left|A_{\{h, i\}}^{\prime}\right|} \leq \frac{v \mathrm{~A}_{\gamma^{\sigma}}\left(A_{\{h, i\}}\right)}{\left|A_{\{h, i\}}\right|}=v \mathrm{~N}_{\gamma^{\sigma}}\left(A_{\{h, i\}}\right) .
$$

Se $A_{\{h, i\}}$ é do tipo 3 , então $v \mathrm{~A}_{\gamma^{\sigma}}\left(A_{\{h, i\}}^{\prime}\right)=v \mathrm{~A}_{\gamma^{\sigma}}\left(A_{\{h, i\}}\right)-2 G$ e $\left|A_{\{h, i\}}^{\prime}\right|=\left|A_{\{h, i\}}\right|-1$ o que implica que

$$
v \mathrm{~N}_{\gamma^{\sigma}}\left(A_{\{h, i\}}^{\prime}\right)=\frac{v \mathrm{~A}_{\gamma^{\sigma}}\left(A_{\{h, i\}}^{\prime}\right)}{\left|A_{\{h, i\}}^{\prime}\right|}=\frac{v \mathrm{~A}_{\gamma^{\sigma}}\left(A_{\{h, i\}}\right)-2 G}{\left|A_{\{h, i\}}\right|-1} \leq v \mathrm{~N}_{\gamma^{\sigma}}\left(A_{\{h, i\}}\right),
$$

onde a última desigualdade é consequência de $v \mathrm{~A}_{\gamma^{\sigma}}\left(A_{\{h, i\}}\right) /\left|A_{\{h, i\}}\right| \leq G$ e $G \geq 1$ desde que, sendo $G$ o valor da maior entrada em $\gamma^{\sigma}, G$ é um limitante superior para $v \mathrm{~N}_{\gamma^{\sigma}}\left(A_{\{h, i\}}\right)$.

Segue de (6.7) e de (6.8) que,

$$
\mathrm{V}_{\gamma^{\sigma}}^{2}\left(A^{\prime}\right)=\sum_{h=1}^{k-1} \sum_{i=h+1}^{k} v \mathrm{~N}_{\gamma^{\sigma}}\left(A_{\{h, i\}}^{\prime}\right) \leq \sum_{h=1}^{k-1} \sum_{i=h+1}^{k} v \mathrm{~N}_{\gamma^{\sigma}}\left(A_{\{h, i\}}\right)=\mathrm{V}_{\gamma^{\sigma}}^{2}(A),
$$

contrariando que $A^{\prime}$ não ser $\mathrm{V}_{\gamma^{\sigma}}^{2}$-ótimo. Logo, existe um alinhamento canônico de $S^{L}$ que é $\mathrm{V}_{\gamma^{\sigma}}^{2}$-ótimo.

Mostramos agora o caso $\eta=3$. Denotamos por $T_{j}$ o conjunto de todos os pares $(h, i)$ tais que $A_{\{h, i\}}$ é do tipo $j$. Lembrando que cada alinhamento induzido $A_{\{h, i\}}$ tipos 1 e 2 são tais que $v \mathrm{~A}_{\gamma^{\sigma}}\left(A_{\{h, i\}}^{\prime}\right) \leq v \mathrm{~A}_{\gamma^{\sigma}}\left(A_{\{h, i\}}\right)$ e $\left|A_{\{h, i\}}^{\prime}\right|=\left|A_{\{h, i\}}\right|$, segue que a contribuição total dos alinhamento induzidos tipos 1 e 2 para a pontuação $\mathrm{V}_{\gamma^{\sigma}}^{3}$ é

$$
\sum_{(h, i) \in T_{1} \cup T_{2}} v \mathrm{~A}_{\gamma^{\sigma}}\left(A_{\{h, i\}}^{\prime}\right) \leq \sum_{(h, i) \in T_{1} \cup T_{2}} v \mathrm{~A}_{\gamma^{\sigma}}\left(A_{\{h, i\}}\right) \quad \text { e } \sum_{(h, i) \in T_{1} \cup T_{2}}\left|A_{\{h, i\}}^{\prime}\right|=\sum_{(h, i) \in T_{1} \cup T_{2}}\left|A_{\{h, i\}}\right| .
$$

E lembrando que cada alinhamento $A_{\{h, i\}}$ do tipo 3 é tal que $v \mathrm{~A}_{\gamma^{\sigma}}\left(A_{\{h, i\}}^{\prime}\right)=v \mathrm{~A}_{\gamma^{\sigma}}\left(A_{\{h, i\}}\right)-2 G$ 
e $\left|A_{\{h, i\}}^{\prime}\right|=\left|A_{\{h, i\}}\right|-1$, temos que

$$
\sum_{(h, i) \in T_{3}} v \mathrm{~A}_{\gamma^{\sigma}}\left(A_{\{h, i\}}^{\prime}\right)=\sum_{(h, i) \in T_{3}}\left(v \mathrm{~A}_{\gamma^{\sigma}}\left(A_{\{h, i\}}\right)-2 G\right) \text { e } \sum_{(h, i) \in T_{3}}\left|A_{\{h, i\}}^{\prime}\right|=\sum_{(h, i) \in T_{3}}\left(\left|A_{\{h, i\}}\right|-1\right) .
$$

Segue daí que

$$
\begin{aligned}
\mathrm{V}_{\gamma^{\sigma}}^{3}\left(A^{\prime}\right) & =\frac{v \mathrm{SP}_{\gamma^{\sigma}}\left(A^{\prime}\right)}{\left|A^{\prime}\right|}=\frac{\sum_{(h, i) \in T_{1} \cup T_{2}} v \mathrm{~A}_{\gamma^{\sigma}}\left(A_{\{h, i\}}^{\prime}\right)+\sum_{(h, i) \in T_{3}} v \mathrm{~A}_{\gamma^{\sigma}}\left(A_{\{h, i\}}^{\prime}\right)}{\sum_{(h, i) \in T_{1} \cup T_{2}}\left|A_{\{h, i\}}^{\prime}\right|+\sum_{(h, i) \in T_{3}}\left|A_{\{h, i\}}^{\prime}\right|} \\
& \leq \frac{\sum_{(h, i) \in T_{1} \cup T_{2}} v \mathrm{~A}_{\gamma^{\sigma}}\left(A_{\{h, i\}}\right)+\sum_{(h, i) \in T_{3}}\left(v \mathrm{~A}_{\gamma^{\sigma}}\left(A_{\{h, i\}}\right)-2 G\right)}{\sum_{(h, i) \in T_{1} \cup T_{2}}\left|A_{\{h, i\}}\right|+\sum_{(h, i) \in T_{3}}\left(\left|A_{\{h, i\}}\right|-1\right)} \\
& =\frac{v \mathrm{SP}_{\gamma^{\sigma}}(A)-2\left|T_{3}\right| G}{|A|-\left|T_{3}\right|} \leq \frac{v \mathrm{SP}_{\gamma^{\sigma}}(A)}{|A|}=\mathrm{V}_{\gamma^{\sigma}}^{3}(A),
\end{aligned}
$$

onde a última desigualdade é consequência de $v \mathrm{SP}_{\gamma^{\sigma}}(A) /|A| \leq G$ e $G \geq 1$. O argumento $v \mathrm{SP}_{\gamma^{\sigma}}(A) /|A| \leq G$ vale pois, sendo $G$ o valor da maior entrada em $\gamma^{\sigma}$, $G$ é um limitante superior para $\mathrm{V}_{\gamma^{\sigma}}^{3}(A)$. Logo, $\mathrm{V}_{\gamma^{\sigma}}^{3}\left(A^{\prime}\right) \leq \mathrm{V}_{\gamma^{\sigma}}^{3}(A)$, contrariando $A^{\prime}$ não ser $\mathrm{V}_{\gamma^{\sigma}}^{3}$-ótimo. Logo, existe um alinhamento canônico de $S^{L}$ que é $\mathrm{V}_{\gamma^{\sigma}}^{3}$-ótimo.

Os próximos resultados mostram que para um valor apropriado para $C^{\eta}$, a solução de uma instância $\left(S^{L}, C^{\eta}\right)$ do Problema AVSN- $\eta$ também é solução da instância $(S, C)$ do Problema AVSd.

Lema 86. Se $C \geq k^{2} M G$, então

$$
\operatorname{AVSd}(S, C)=\operatorname{AVSN}-1\left(S^{L}, \frac{C}{L}\right)=\operatorname{AVSN}-2\left(S^{L}, \frac{C}{L}\right)=\operatorname{AVSN}-3\left(S^{L}, \frac{C}{\left(\begin{array}{l}
k \\
2
\end{array}\right) L}\right)=\operatorname{Sim} .
$$

Prova. Suponha que $C \geq k^{2} M G$. Seja $A$ um alinhamento de $S$. Segue do Fato 84 que $v \mathrm{SP}_{\gamma}(A)=v \mathrm{SP}_{\gamma^{\sigma}}\left(A^{L}\right) \leq k^{2} M G \leq C$.

Como $v \operatorname{SP}_{\gamma}(A) \leq C$, temos que $\operatorname{AVSd}(S, C)=\operatorname{Sim}$. Como $v \mathrm{SP}_{\gamma^{\sigma}}\left(A^{L}\right) \leq C$, temos que $\mathrm{V}_{\gamma^{\sigma}}^{1}\left(A^{L}\right)=v \mathrm{SP}_{\gamma^{\sigma}}\left(A^{L}\right) / L \leq C / L$, o que implica que $\operatorname{AVSN}-1\left(S^{L}, \frac{C}{L}\right)=\operatorname{Sim}$. Como $v \mathrm{SP}_{\gamma^{\sigma}}\left(A^{L}\right) \leq C$ e $\left|A_{\{h, i\}}^{L}\right| \geq L$, temos que

$$
\mathrm{V}_{\gamma^{\sigma}}^{2}\left(A^{L}\right)=\sum_{h=1}^{k-1} \sum_{i=h+1}^{k} \frac{v \mathrm{~A}_{\gamma^{\sigma}}\left(A_{\{h, i\}}^{L}\right)}{\left|A_{\{h, i\}}^{L}\right|} \leq \frac{\sum_{h=1}^{k-1} \sum_{i=h+1}^{k} v \mathrm{~A}_{\gamma^{\sigma}}\left(A_{\{h, i\}}^{L}\right)}{L} \leq \frac{C}{L},
$$

o que implica que AVSN-2 $\left(S^{L}, \frac{C}{L}\right)=\operatorname{Sim} . \operatorname{E} v \operatorname{SP}_{\gamma^{\sigma}}\left(A^{L}\right) \leq C$ e $\left|A_{\{h, i\}}^{L}\right| \geq L$ também implica que

$$
\mathrm{V}_{\gamma^{\sigma}}^{3}\left(A^{L}\right)=\frac{v \mathrm{SP}_{\gamma^{\sigma}}\left(A^{L}\right)}{\sum_{h=1}^{k-1} \sum_{i=h+1}^{k}\left|A_{\{h, i\}}^{L}\right|} \leq \frac{v \mathrm{SP}_{\gamma^{\sigma}}\left(A^{L}\right)}{\sum_{h=1}^{k-1} \sum_{i=h+1}^{k} L}=\frac{v \mathrm{SP}_{\gamma^{\sigma}}\left(A^{L}\right)}{\left(\begin{array}{c}
k \\
2
\end{array}\right) L} \leq \frac{C}{\left(\begin{array}{c}
k \\
2
\end{array}\right) L}
$$

o que implica que AVSN-3 $\left(S^{L}, \frac{C}{\left(\begin{array}{c}k \\ 2\end{array}\right) L}\right)=\operatorname{Sim}$.

Portanto, se $C \geq k^{2} M G$, então

$\operatorname{AVSd}(S, C)=\operatorname{AVSN}-1\left(S^{L}, \frac{C}{L}\right)=\operatorname{AVSN}-2\left(S^{L}, \frac{C}{L}\right)=\operatorname{AVSN}-3\left(S^{L}, \frac{C}{\left(\begin{array}{c}k \\ 2\end{array}\right) L}\right)=\operatorname{Sim}$ 
Lema 87. Sejam $C^{1}=C^{2}=C / L$ e $C^{3}=C /\left(\left(\begin{array}{l}k \\ 2\end{array}\right) L\right)$ e $L=N k^{2} M G$. Então, $\operatorname{AVSd}(S, C)=$ Sim se e somente se $\operatorname{AVSN}-\eta\left(S^{L}, C^{\eta}\right)=$ Sim para cada $\eta$.

Prova. Suponha que $C \geq k^{2} M G$. Como consequência do Lema 86, temos que o lema está provado. Assumimos então que $C<k^{2} M G$ e para provar o lema vamos mostrar que valem as seguintes afirmações quando $C<k^{2} M G$ : $\operatorname{se} \operatorname{AVSd}(S, C)=\operatorname{Sim}$, então

$$
\operatorname{AVSN}-1\left(S^{L}, \frac{C}{L}\right)=\operatorname{AVSN}-2\left(S^{L}, \frac{C}{L}\right)=\operatorname{AVSN}-3\left(S^{L}, \frac{C}{\left(\begin{array}{c}
k \\
2
\end{array}\right) L}\right)=\operatorname{Sim}
$$

e, para cada $\eta$, vale que se $\operatorname{AVSN-} \eta\left(S^{L}, C^{\eta}\right)=\operatorname{Sim}$, então $\operatorname{AVSd}(S, C)=\operatorname{Sim}$.

Suponha então que $\operatorname{AVSd}(S, C)=\operatorname{Sim}$. Logo, existe um alinhamento $A$ de $S$ tal que $v \operatorname{SP}_{\gamma}(A) \leq C$. Sendo $v \mathrm{SP}_{\gamma^{\sigma}}\left(A^{L}\right)=v \mathrm{SP}_{\gamma}(A)$, segue que

$$
\begin{aligned}
\mathrm{V}_{\gamma^{\sigma}}^{1}\left(A^{L}\right) & =\frac{v \mathrm{SP}_{\gamma^{\sigma}}\left(A^{L}\right)}{\left|A^{L}\right|} \leq \frac{v \mathrm{SP}_{\gamma^{\sigma}}\left(A^{L}\right)}{L}=\frac{v \mathrm{SP}_{\gamma}(A)}{L} \leq \frac{C}{L} \\
\mathrm{~V}_{\gamma^{\sigma}}^{2}\left(A^{L}\right) & =\sum_{h=1}^{k-1} \sum_{i=h+1}^{k} \frac{v \mathrm{~A}_{\gamma^{\sigma}}\left(A_{\{h, i\}}^{L}\right)}{\left|A_{\{h, i\}}^{L}\right|} \\
& \leq \sum_{h=1}^{k-1} \sum_{i=h+1}^{k} \frac{v \mathrm{~A}_{\gamma^{\sigma}}\left(A_{\{h, i\}}^{L}\right)}{L}=\frac{v \mathrm{SP}_{\gamma^{\sigma}}\left(A^{L}\right)}{L}=\frac{v \mathrm{SP}_{\gamma}(A)}{L} \leq \frac{C}{L} \\
\mathrm{~V}_{\gamma^{\sigma}}^{3}\left(A^{L}\right) & =\frac{v \mathrm{SP}_{\gamma^{\sigma}}\left(A^{L}\right)}{\sum_{h=1}^{k-1} \sum_{i=h+1}^{k}\left|A_{\{h, i\}}^{L}\right|} \leq \frac{v \mathrm{SP}_{\gamma^{\sigma}}\left(A^{L}\right)}{\sum_{h=1}^{k-1} \sum_{i=h+1}^{k} L}=\frac{v \mathrm{SP}_{\gamma^{\sigma}}\left(A^{L}\right)}{\left(\begin{array}{c}
k \\
2
\end{array}\right) L}=\frac{v \mathrm{SP}_{\gamma}(A)}{\left(\begin{array}{c}
k \\
2
\end{array}\right) L} \leq \frac{C}{\left(\begin{array}{c}
k \\
2
\end{array}\right) L}
\end{aligned}
$$

onde a primeira desigualdade de cada expressão segue de que $A^{L}$ ou cada alinhamento induzido de $A^{L}$ possui comprimento maior ou igual a $L$, e a segunda desigualdade segue de $v \operatorname{SP}_{\gamma}(A) \leq C$. Segue que se $\operatorname{AVSd}(S, C)=\operatorname{Sim}$, então

$$
\operatorname{AVSN}-1\left(S^{L}, \frac{C}{L}\right)=\operatorname{AVSN}-2\left(S^{L}, \frac{C}{L}\right)=\operatorname{AVSN}-3\left(S^{L}, \frac{C}{\left(\begin{array}{c}
k \\
2
\end{array}\right) L}\right)=\operatorname{Sim}
$$

Suponha que AVSN- $i\left(S^{L}, C^{\eta}\right)=$ Sim. Escolha um alinhamento $\mathrm{V}_{\gamma^{\sigma}}^{\eta}$-ótimo e canônico $A^{L}$ de $S^{L}$ que o Lema 85 garante existir. Segue que $\mathrm{V}_{\gamma^{\sigma}}^{\eta}\left(A^{L}\right) \leq C^{\eta}$.

Então, o alinhamento $A^{L}$ é tal que $\mathrm{V}_{\gamma^{\sigma}}^{1}\left(A^{L}\right) \leq C^{1}=C / L$. Segue que

$$
\begin{aligned}
v \mathrm{SP}_{\gamma}(A) & =v \mathrm{SP}_{\gamma^{\sigma}}\left(A^{L}\right)=(N+L) \cdot \frac{v \mathrm{SP}_{\gamma^{\sigma}}\left(A^{L}\right)}{N+L} \\
& \leq(N+L) \cdot \frac{v \mathrm{SP}_{\gamma^{\sigma}}\left(A^{L}\right)}{\left|A^{L}\right|}=(N+L) \cdot \mathrm{V}_{\gamma^{\sigma}}^{1}\left(A^{L}\right) \\
& \leq(N+L) \cdot \frac{C}{L}=\frac{N C}{L}+C \leq \frac{N k^{2} M G}{L}+C<1+C,
\end{aligned}
$$

onde (6.9) segue de que $\left|A^{L}\right| \leq N+L$. 
O alinhamento $A^{L}$ é tal que $\bigvee_{\gamma^{\sigma}}^{2}\left(A^{L}\right) \leq C^{2}=C / L$. Segue que

$$
\begin{aligned}
v \mathrm{SP}_{\gamma}(A) & =v \mathrm{SP}_{\gamma^{\sigma}}\left(A^{L}\right)=(N+L) \cdot \frac{v \mathrm{SP}_{\gamma^{\sigma}}\left(A^{L}\right)}{N+L}=(N+L) \cdot \sum_{h=1}^{k-1} \sum_{i=h+1}^{k} \frac{v \mathrm{~A}_{\gamma^{\sigma}}\left(A_{\{h, i\}}^{L}\right)}{N+L} \\
& \leq(N+L) \cdot \sum_{h=1}^{k-1} \sum_{i=h+1}^{k} \frac{v \mathrm{~A}_{\gamma^{\sigma}}\left(A_{\{h, i\}}^{L}\right)}{\left|A_{\{h, i\}}^{L}\right|}=(N+L) \cdot \mathrm{V}_{\gamma^{\sigma}}^{2}\left(A^{L}\right) \\
& \leq(N+L) \cdot \frac{C}{L}=\frac{N C}{L}+C \leq \frac{N k^{2} M G}{L}+C<1+C,
\end{aligned}
$$

onde (6.10) segue de que o comprimento de qualquer alinhamento induzido de um alinhamento canônico não é maior do que $N+L$.

O alinhamento $A^{L}$ é tal que $\mathrm{V}_{\gamma^{\sigma}}^{3}\left(A^{L}\right) \leq C^{3}=C /\left(L\left(\begin{array}{l}k \\ 2\end{array}\right)\right)$. Segue que

$$
\begin{aligned}
v \mathrm{SP}_{\gamma}(A) & =v \mathrm{SP}_{\gamma^{\sigma}}\left(A^{L}\right)=\left(N+L\left(\begin{array}{l}
k \\
2
\end{array}\right)\right) \frac{v \mathrm{SP}_{\gamma^{\sigma}}\left(A^{L}\right)}{N+L\left(\begin{array}{l}
k \\
2
\end{array}\right)} \\
& \leq\left(N+L\left(\begin{array}{l}
k \\
2
\end{array}\right)\right) \frac{v \mathrm{SP}_{\gamma^{\sigma}}\left(A^{L}\right)}{\sum_{h=1}^{k-1} \sum_{i=h+1}^{k}\left|A_{\{h, i\}}^{L}\right|}=\left(N+L\left(\begin{array}{l}
k \\
2
\end{array}\right)\right) \mathrm{V}_{\gamma^{\sigma}}^{3}\left(A^{L}\right) \\
& \leq\left(N+L\left(\begin{array}{l}
k \\
2
\end{array}\right)\right) \frac{C}{L\left(\begin{array}{c}
k \\
2
\end{array}\right)}=\frac{N C}{L\left(\begin{array}{c}
k \\
2
\end{array}\right)}+C<\frac{N k^{2} M G}{\left(\begin{array}{l}
k \\
2
\end{array}\right) L}+C<1+C,
\end{aligned}
$$

onde a desigualdade em (6.11) segue de que a soma dos comprimentos dos alinhamentos de duas sequências induzidos de um alinhamento canônico não é maior do que $N+L\left(\begin{array}{l}k \\ 2\end{array}\right)$.

Portanto, considerando qualquer $\eta$, temos que $\operatorname{AVSN}-i\left(S^{L}, C^{\eta}\right)=$ Sim implica que $v \mathrm{SP}_{\gamma}(A)<1+C$. Como as entradas da matriz de pontuação são inteiras segue que $v \mathrm{SP}_{\gamma}(A)$ é um número inteiro. Sendo $C$ um número inteiro, segue que $v \operatorname{SP}_{\gamma}(A) \leq C$.

\subsection{Algoritmo de Aproximação}

Gusfield [Gus93] descreve um algoritmo que é uma 2-aproximação para o Problema AVS. Seu algoritmo leva em consideração que $\gamma \in \mathbb{M}^{\mathrm{C}}$, ou seja, a matriz de pontuação utilizada é uma métrica em $\Sigma_{\text {b }}$ e a 2-aproximação é obtida porque vale $\gamma_{a \rightarrow c} \leq \gamma_{a \rightarrow b}+\gamma_{b \rightarrow c}$ para cada $\mathrm{a}, \mathrm{b}, \mathrm{c} \in \Sigma$. Nesta seção, adaptamos o algoritmo de Gusfield e descrevemos um algoritmo que é uma 6-aproximação para o Problema AVS quando $\gamma \in \mathbb{M}^{\mathrm{A}}$ e é uma 12-aproximação para a versão de otimização do Problema AVSN-2 quando $\gamma \in \mathbb{M}^{\mathbb{N}}$. A versão de otimização de AVSN-2 tem como objetivo, dado uma $k$-upla de sequências $S$, encontrar $\mathrm{OPT}_{\gamma}^{2}(S)$ para uma matriz de pontuação fixa $\gamma$.

Conforme visto no Capítulo 4 , vale $\gamma \in \mathbb{M}^{\mathrm{A}}$ se e somente se

1. $\gamma_{\mathrm{a} \rightarrow \sqcup}=\gamma_{\sqcup \rightarrow \mathrm{a}}>0$,

2. $\gamma_{\mathrm{a} \rightarrow \mathrm{b}}>0$ se $\mathrm{a} \neq \mathrm{b}$, e $\gamma_{\mathrm{a} \rightarrow \mathrm{b}}=0$ se $\mathrm{a}=\mathrm{b}$,

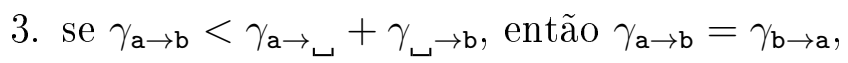

4. $\gamma_{\mathrm{a} \rightarrow \sqcup} \leq \gamma_{\mathrm{a} \rightarrow \mathrm{b}}+\gamma_{\mathrm{b} \rightarrow \sqcup}$,

5. $\min \left\{\gamma_{\mathrm{a} \rightarrow \mathrm{c}}, \gamma_{\mathrm{a} \rightarrow \sqcup}+\gamma_{\lrcorner \rightarrow \mathrm{c}}\right\} \leq \gamma_{\mathrm{a} \rightarrow \mathrm{b}}+\gamma_{\mathrm{b} \rightarrow \mathrm{c}}$, 
e $\gamma \in \mathbb{M}^{\mathbb{N}}$ se e somente se

1. $\gamma \in \mathbb{M}^{\mathrm{A}} \mathrm{e}$

2. $\gamma_{\mathrm{a} \rightarrow \sqcup} \leq 2 \gamma_{\mathrm{b} \rightarrow_{\sqcup}}$ para cada $\mathrm{a}, \mathrm{b} \in \Sigma$.

\subsection{1 v-estrela}

Sejam $S=\left(s_{1}, \ldots, s_{k}\right)$ uma $k$-upla de sequências, $v$ uma função genérica para pontuar um alinhamento de duas sequências tal que $v\left(\left[s_{h}^{\prime}, s_{h}^{\prime}\right]\right)=0$, para qualquer alinhamento de $\left(s_{h}, s_{h}\right), c$ um inteiro tal que $1 \leq c \leq k$ e A uma coleção de $k-1$ alinhamentos: um alinhamento $\mathrm{A}_{h}$ de $\left(s_{h}, s_{c}\right)$ para cada $h<c$, e de $\left(s_{c}, s_{h}\right)$ para cada $h>c$. A v-estrela de $S$ com centro $c$ para $\mathrm{A}$ é um digrafo $D(\mathrm{~A}, c)$ cujo conjunto de vértices é $\{1, \ldots, k\}$ e o conjunto de $\operatorname{arcos}$ é

$$
\{h \rightarrow c: h=1,2, \ldots, c-1\} \cup\{c \rightarrow h: h=c+1, c+2, \ldots, k\} .
$$

Cada arco $h \rightarrow c$ e cada arco $c \rightarrow h$ tem custo $v\left(\mathrm{~A}_{h}\right)$.
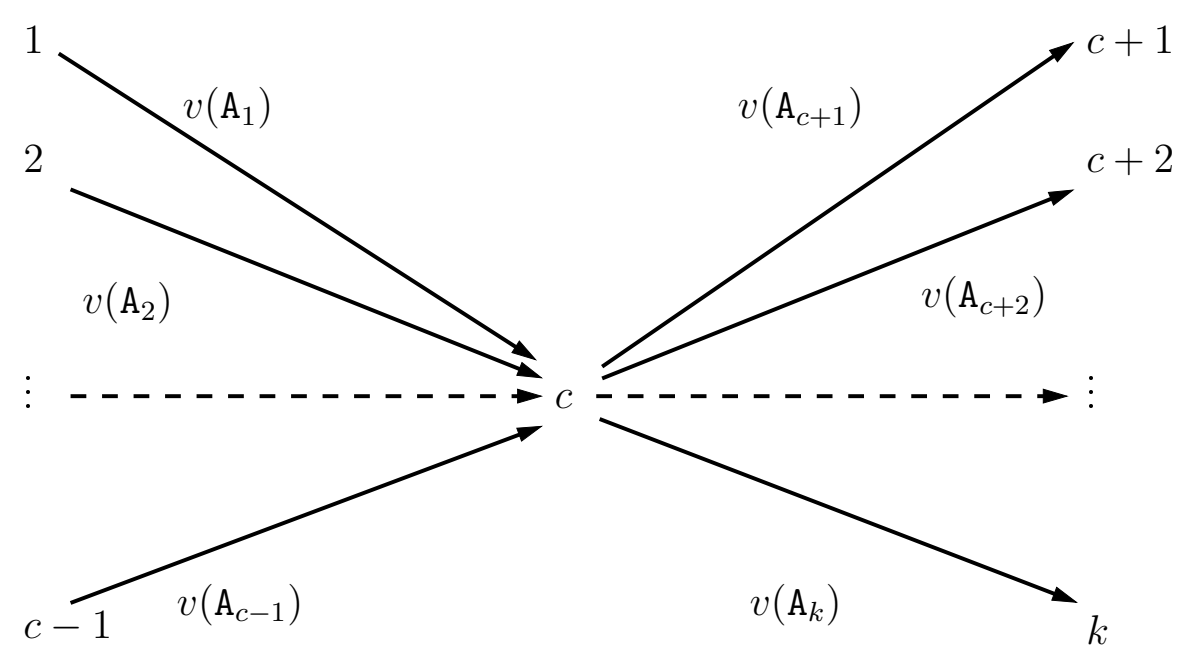

O custo do digrafo $D(\mathrm{~A}, c)$, que denotamos custo $(D(\mathrm{~A}, c))$, é a soma dos custos dos arcos de $D(\mathrm{~A}, c)$, ou seja, custo $(D(\mathrm{~A}, c))=\sum_{h \neq c} v\left(\mathrm{~A}_{h}\right)$. Observe que se A é tal que, para um alinhamento $A, \mathrm{~A}_{h}=A_{\{c, h\}}$ para todo $\mathrm{A}_{h} \in \mathrm{A}$, então

$$
\sum_{h=1}^{k-1} \sum_{i=h+1}^{k}\left(v\left(A_{\{h, c\}}\right)+v\left(A_{\{c, i\}}\right)\right)=(k-1) \cdot \operatorname{custo}(D(\mathrm{~A}, c)) .
$$

Uma $v$-estrela $D(\mathrm{~A}, c)$ de $S$ é ótima se não existe uma $v$-estrela de $S$ de custo menor que $\operatorname{custo}(D(\mathrm{~A}, c))$. Um alinhamento $\left[s_{h}^{\prime}, s_{i}^{\prime}\right]$ de $\left(s_{h}, s_{i}\right)$ é $v$-ótimo se não existe alinhamento de $\left(s_{h}, s_{i}\right)$ de custo menor do que $v\left(\left[s_{h}^{\prime}, s_{i}^{\prime}\right]\right)$. Denotamos custo de um alinhamento $v$-ótimo de $\left(s_{h}, s_{i}\right)$ por opt $\left(s_{h}, s_{i}\right)$. Note que o custo de uma $v$-estrela ótima é

$$
\min _{c}\left\{\sum_{h=1}^{c-1} \operatorname{opt}\left(s_{h}, s_{c}\right)+\sum_{h=c+1}^{k} \operatorname{opt}\left(s_{c}, s_{h}\right)\right\} .
$$

Para um alinhamento $A$ de uma $k$-upla $S$, denotamos $V(A)=\sum_{h=1}^{k-1} \sum_{i=h+1}^{k} v\left(A_{\{h, i\}}\right)$ e $\mathrm{OPT}(S)=\min _{A \in \mathcal{A}_{S}}\{V(A)\}$. Se $A$ é um alinhamento e $V(A)=\operatorname{OPT}(S)$, dizemos que $A$ é um alinhamento $V$-ótimo. 
Lema 88. Se $D(\mathrm{~A}, c)$ é uma $v$-estrela ótima, então

$$
\operatorname{custo}(D(\mathrm{~A}, c)) \leq \frac{2}{k} \cdot \mathrm{OPT}(S)
$$

Prova. Seja $A$ um alinhamento $V$-ótimo e suponha que $D(\mathrm{~A}, c)$ é uma $v$-estrela ótima. Então,

$$
\begin{aligned}
\frac{k \cdot(k-1)}{2} \cdot \operatorname{custo}(D(\mathrm{~A}, c)) & =\frac{k-1}{2} \cdot \sum_{i=1}^{k} \operatorname{custo}(D(\mathrm{~A}, c)) \leq \frac{k-1}{2} \cdot \sum_{i=1}^{k} \sum_{h \neq i} v\left(A_{\{h, i\}}\right) \\
& =\frac{k-1}{2} \cdot(2 V(A))=(k-1) \cdot \operatorname{OPT}(S),
\end{aligned}
$$

onde a desigualdade segue da otimalidade da $v$-estrela $D(\mathrm{~A}, c)$. Segue que se $D(\mathrm{~A}, c)$ é uma $v$-estrela ótima, então custo $(D(\mathrm{~A}, c)) \leq(2 / k) \cdot \mathrm{OPT}(S)$.

\subsubsection{Desdobramento}

Sejam $s, t \in \Sigma^{*}$. Suponha que $A=\left[s^{\prime}, t^{\prime}\right]$ é alinhamento de $(s, t)$ ou $A=\left[t^{\prime}, s^{\prime}\right]$ é alinhamento de $(t, s)$. Dizemos que uma coluna $j$ de $A$ é $s$-desdobrável se $s^{\prime}(j) \neq \sqcup, t^{\prime}(j) \neq \sqcup$ e $\gamma_{t^{\prime}(j) \rightarrow_{\sqcup}} \leq \gamma_{s^{\prime}(j) \rightarrow t^{\prime}(j)}$. Seja $J=\left\{j_{1}, \ldots, j_{m}\right\}$, com $1 \leq j_{1}<j_{2}<\ldots<j_{m} \leq|A|$, o conjunto de todos os inteiros que são colunas s-desdobráveis de $A$. O s-desdobramento do alinhamento $A$ é o alinhamento

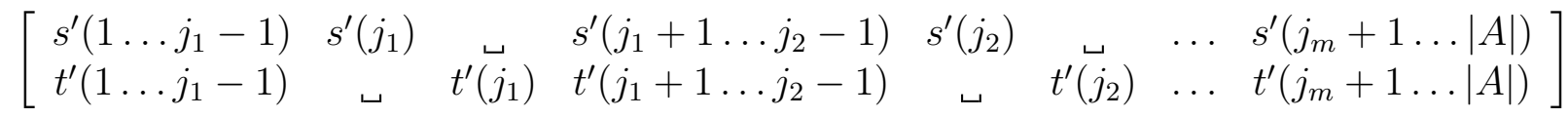

se $A=\left[s^{\prime}, t^{\prime}\right]$ e

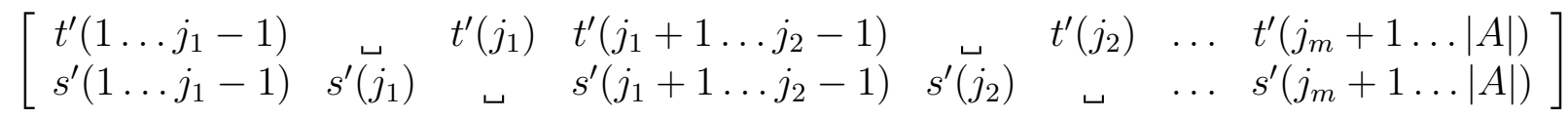

se $A=\left[t^{\prime}, s^{\prime}\right]$. Dizemos que $J$ é o conjunto que define o $s$-desdobramento de $A$.

O seguinte fato é usado para auxiliar o cálculo da pontuação de um $s$-desdobramento.

Fato 89. Seja $\gamma \in \mathbb{M}^{\mathrm{A}}$ e $\mathrm{a}, \mathrm{b} \in \Sigma$. Então, $\gamma_{\mathrm{a} \rightarrow_{\sqcup}}>\gamma_{\mathrm{a} \rightarrow \mathrm{b}}$ se e somente se $\gamma_{\mathrm{a} \rightarrow_{\sqcup}}>\gamma_{\mathrm{b} \rightarrow \mathrm{a}}$.

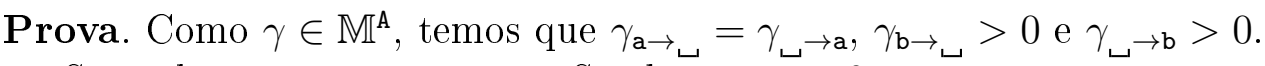

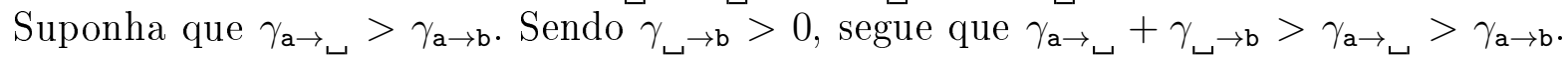
Logo $\gamma_{a \rightarrow}+\gamma_{b \rightarrow b}>\gamma_{a \rightarrow b}$ o que implica, sendo $\gamma \in \mathbb{M}^{A}$, que $\gamma_{a \rightarrow b}=\gamma_{b \rightarrow a}$. Logo, $\gamma_{a \rightarrow}>$

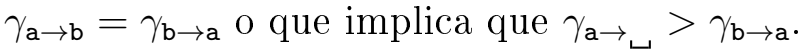

Suponha agora que $\gamma_{\mathrm{a} \rightarrow_{\sqcup}}>\gamma_{\mathrm{b} \rightarrow \mathrm{a}}$. Desde que $\gamma_{\mathrm{a} \rightarrow_{\sqcup}}=\gamma_{\mathrm{b}_{\mathrm{a}}}$ e $\gamma_{\mathrm{b} \rightarrow_{\sqcup}}>0$, segue que

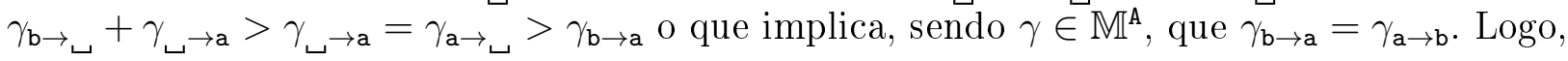
$\gamma_{\mathrm{a} \rightarrow\llcorner}>\gamma_{\mathrm{b} \rightarrow \mathrm{a}}=\gamma_{\mathrm{a} \rightarrow \mathrm{b}}$

O c-desdobramento de uma $v$-estrela $D(\mathrm{~A}, c)$ de $S=\left(s_{1}, \ldots, s_{k}\right)$ é a $v$-estrela $D\left(\mathrm{~A}^{c}, c\right)$ de $S$ onde os alinhamentos em $\mathrm{A}^{c}$ são $s_{c}$-desdobramentos dos alinhamentos em A. O próximo resultado mostra que o $c$-desdobramento de uma $v$-estrela aumenta o seu custo de maneira limitada quando $\gamma \in \mathbb{M}^{\mathrm{A}}$ e $v=v \mathrm{~A}_{\gamma}$ ou $v=v \mathrm{~N}_{\gamma}$. 
Proposição 90. Sejam $S=\left(s_{1}, \ldots, s_{k}\right)$ uma $k$-upla de sequências, $c$ um inteiro e $D(\mathrm{~A}, c)$ uma $v$-estrela de $S$. Considere $\gamma \in \mathbb{M}^{\mathrm{A}}$ e $v=v \mathrm{~A}_{\gamma}$ ou $v=v \mathbf{N}_{\gamma}$. Então,

$$
\operatorname{custo}\left(D\left(\mathrm{~A}^{c}, c\right)\right) \leq 3 \cdot \operatorname{custo}(D(\mathrm{~A}, c)) \text {. }
$$

Prova. Sejam $h$ um inteiro arbitrário, $1 \leq h<c, \mathrm{~A}_{h}=\left[s_{h}^{\prime}, s_{c}^{\prime}\right] \in \mathrm{A}$ alinhamento de $\left(s_{h}, s_{c}\right)$ e $\left[s_{h}^{\prime \prime}, s_{c}^{\prime \prime}\right]=\mathrm{A}_{h}^{c}$. Considere também $J$ o conjunto que define o $s_{c}$-desdobramento de $\left[s_{h}^{\prime}, s_{c}^{\prime}\right]$. Segue que

$$
\begin{aligned}
v \mathrm{~A}_{\gamma}\left(\left[s_{h}^{\prime \prime}, s_{c}^{\prime \prime}\right]\right) & =v \mathrm{~A}_{\gamma}\left(\left[s_{h}^{\prime}, s_{c}^{\prime}\right]\right)+\sum_{j \in J}\left(\gamma_{s_{h}^{\prime}(j) \rightarrow \sqcup}+\gamma_{\sqcup \rightarrow s_{c}^{\prime}(j)}-\gamma_{s_{h}^{\prime}(j) \rightarrow s_{c}^{\prime}(j)}\right) \\
& \leq v \mathrm{~A}_{\gamma}\left(\left[s_{h}^{\prime}, s_{c}^{\prime}\right]\right)+\sum_{j \in J}\left(\gamma_{s_{h}^{\prime}(j) \rightarrow \sqcup}+\gamma_{\sqcup \rightarrow s_{h}^{\prime}(j)}\right) \\
& =v \mathrm{~A}_{\gamma}\left(\left[s_{h}^{\prime}, s_{c}^{\prime}\right]\right)+2 \cdot \sum_{j \in J} \gamma_{s_{h}^{\prime}(j) \rightarrow \sqcup} \\
& \leq v \mathrm{~A}_{\gamma}\left(\left[s_{h}^{\prime}, s_{c}^{\prime}\right]\right)+2 \cdot \sum_{j \in J} \gamma_{s_{h}^{\prime}(j) \rightarrow s_{c}^{\prime}(j)},
\end{aligned}
$$

onde (6.13) e (6.14) valem porque $\gamma \in \mathbb{M}^{\mathrm{A}}$; e (6.15) vale pelo Fato 89 desde que, sendo cada $j \in J$ um $s_{c}$-desdobramento de $\left[s_{h}^{\prime}, s_{c}^{\prime}\right], \gamma_{s_{h}^{\prime}(j) \rightarrow_{\sqcup}} \leq \gamma_{s_{c}^{\prime}(j) \rightarrow s_{h}^{\prime}(j)}$ por definição.

Desde que $J \subseteq\{1,2, \ldots,|A|\}$ e $\gamma \in \mathbb{M}^{\mathrm{A}}$, temos que

$$
\sum_{j \in J} \gamma_{s_{h}^{\prime}(j) \rightarrow s_{c}^{\prime}(j)} \leq \sum_{j \in\{1,2, \ldots,|A|\}} \gamma_{s_{h}^{\prime}(j) \rightarrow s_{c}^{\prime}(j)}=v \mathrm{~A}_{\gamma}\left(\left[s_{h}^{\prime}, s_{c}^{\prime}\right]\right)
$$

Segue que

$$
\begin{aligned}
v \mathrm{~A}_{\gamma}\left(\left[s_{h}^{\prime \prime}, s_{c}^{\prime \prime}\right]\right) & \leq v \mathrm{~A}_{\gamma}\left(\left[s_{h}^{\prime}, s_{c}^{\prime}\right]\right)+2 \cdot \sum_{j \in J} \gamma_{s_{h}^{\prime}(j) \rightarrow s_{c}^{\prime}(j)} \\
& \leq v \mathrm{~A}_{\gamma}\left(\left[s_{h}^{\prime}, s_{c}^{\prime}\right]\right)+2 \cdot v \mathrm{~A}_{\gamma}\left(\left[s_{h}^{\prime}, s_{c}^{\prime}\right]\right)=3 \cdot v \mathrm{~A}_{\gamma}\left(\left[s_{h}^{\prime}, s_{c}^{\prime}\right]\right) .
\end{aligned}
$$

Além disso,

$$
\begin{aligned}
v \mathrm{~N}_{\gamma}\left(\left[s_{h}^{\prime \prime}, s_{c}^{\prime \prime}\right]\right) & =\frac{v \mathrm{~A}_{\gamma}\left(\left[s_{h}^{\prime \prime}, s_{c}^{\prime \prime}\right]\right)}{\left|\left[s_{h}^{\prime \prime}, s_{c}^{\prime \prime}\right]\right|} \leq \frac{3 \cdot v \mathrm{~A}_{\gamma}\left(\left[s_{h}^{\prime}, s_{c}^{\prime}\right]\right)}{\left|\left[s_{h}^{\prime}, s_{c}^{\prime}\right]\right|+|J|} \leq \frac{3 \cdot v \mathrm{~A}_{\gamma}\left(\left[s_{h}^{\prime}, s_{c}^{\prime}\right]\right)}{\left|\left[s_{h}^{\prime}, s_{c}^{\prime}\right]\right|} \\
& =3 \cdot v \mathrm{~N}_{\gamma}\left(\left[s_{h}^{\prime}, s_{c}^{\prime}\right]\right) .
\end{aligned}
$$

Portanto, desde que o argumento vale para um inteiro $h$ arbitrário, temos que se $h<c$, então $v\left(\mathrm{~A}_{h}^{c}\right)=v\left(\left[s_{h}^{\prime \prime}, s_{c}^{\prime \prime}\right]\right) \leq 3 \cdot v\left(\left[s_{h}^{\prime}, s_{c}^{\prime}\right]\right)=3 \cdot v\left(\mathrm{~A}_{h}\right)$. Usando argumentos similares, temos também que se $h>c$, então $v\left(\mathrm{~A}_{h}^{c}\right) \leq 3 \cdot v\left(\mathrm{~A}_{h}\right)$. Logo,

$$
\operatorname{custo}\left(D\left(\mathrm{~A}^{c}, c\right)\right)=\sum_{h<c} v\left(\mathrm{~A}_{h}^{c}\right)+\sum_{h>c} v\left(\mathrm{~A}_{h}^{c}\right)=\sum_{h \neq c} v\left(\mathrm{~A}_{h}^{c}\right) \leq 3 \cdot \sum_{h \neq c} v\left(\mathrm{~A}_{h}\right)=3 \cdot \operatorname{custo}(D(\mathrm{~A}, c)) .
$$

O próximo lema mostra uma relação entre o desdobramento de $v$-estrelas ótimas e alinhamentos $v$-ótimos quando $\gamma \in \mathbb{M}^{\mathrm{A}}$ e $v=v \mathrm{~A}_{\gamma}$ ou $v=v \mathrm{~N}_{\gamma}$.

Lema 91. Sejam $\gamma \in \mathbb{M}^{\mathrm{A}}, v=v \mathrm{~A}_{\gamma}$ ou $v=v \mathrm{~N}_{\gamma}, D(\mathrm{~A}, c)$ uma $v$-estrela ótima de $S$, e $A$ um 
alinhamento de $S$ tal que $A_{\{c, h\}}=\mathrm{A}_{h}^{c}$ para cada $\mathrm{A}_{h}^{c} \in \mathrm{A}^{c}$. Então,

$$
\sum_{h=1}^{k-1} \sum_{i=h+1}^{k}\left(v\left(A_{\{h, c\}}\right)+v\left(A_{\{c, i\}}\right)\right) \leq 6 \cdot \operatorname{OPT}(S)
$$

Prova. Temos que

$$
\begin{aligned}
\sum_{h=1}^{k-1} \sum_{i=h+1}^{k}\left(v\left(A_{\{h, c\}}\right)+v\left(A_{\{c, i\}}\right)\right) & =(k-1) \cdot \operatorname{custo}\left(D\left(\mathrm{~A}^{c}, c\right)\right) \\
& \leq 3 \cdot(k-1) \cdot \operatorname{custo}(D(\mathrm{~A}, c)) \\
& \leq 3 \cdot(k-1) \cdot \frac{2}{k} \cdot \mathrm{OPT}(S) \leq 6 \cdot \operatorname{OPT}(S)
\end{aligned}
$$

onde (6.16) segue de (6.12), (6.17) segue da Proposição 90 e (6.18) segue do Lema 88.

Note que o tempo gasto para computar um $s_{c}$-desdobramento depende de qual estrutura de dados utilizamos para representar o alinhamento. Se utilizamos uma matriz para representar um alinhamento, o tempo gasto não é superior à quantidade de elementos do alinhamento que é $O\left(k^{2} n\right)$.

\subsubsection{Alinhamento compatível}

Um alinhamento $A$ é dito compatível com uma $v$-estrela $D(\mathrm{~A}, c)$ se $A_{\{h, c\}}=\mathrm{A}_{h}$ para cada $h \neq c$. Há vários alinhamentos compatíveis com uma $v$-estrela de $S$. Descrevemos a seguir e imerso no texto o Algoritmo AlinhaCompativel que recebe uma $v$-estrela $D(\mathrm{~A}, c)$ e devolve um alinhamento $A$ compatível com a $v$-estrela $D(\mathrm{~A}, c)$ e que possui propriedades que são necessárias para garantir os fatores de aproximação tanto para o problema AVS quanto para a versão de otimização do Problema AVSN-2.

Considere

$$
\begin{aligned}
\mathrm{A}= & \left\{\left[s_{h}^{\prime}, s_{c}^{h}\right]: \text { alinhamento de }\left(s_{h}, s_{c}\right) \text { para } h<c\right\} \cup \\
& \left\{\left[s_{c}^{h}, s_{h}^{\prime}\right]: \text { alinhamento de }\left(s_{c}, s_{h}\right) \text { para } h>c\right\} .
\end{aligned}
$$

Para $h \neq c, 1 \leq h \leq k, R(h, 0)$ denota a quantidade de 's antes de $s_{c}(1)$ em $s_{c}^{h}, R(h, j)$ a quantidade de ${ }^{\prime}$ 's entre $s_{c}(j)$ e $s_{c}(j+1)$ para $j=1,2, \ldots,\left|s_{c}\right|-1$ e $R\left(h,\left|s_{c}\right|\right)$ a quantidade de ${ }^{\prime}$ 's depois de $s_{c}\left(\left|s_{c}\right|\right)$ em $s_{c}^{h}$. Com essa definição podemos escrever cada $s_{c}^{h}$ como

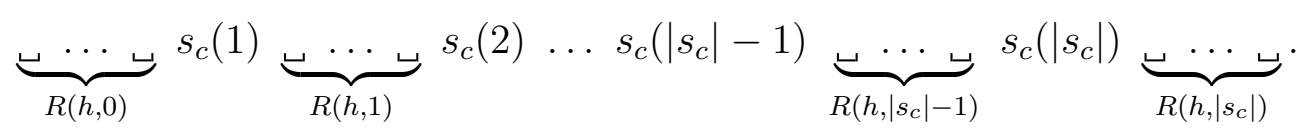

Definimos também para $j=0, \ldots,\left|s_{c}\right|$ e $h=1, \ldots, k+1, h \neq c$

$$
Q(h, j)=\sum_{i=0}^{h-1} R(i, j)
$$

Por exemplo, para $S=$ (aaa, bbbbb, cc, ddd, eeeeee), considere

$$
A=\left\{\left[\begin{array}{cccc}
a & a & a & u \\
& d & d & d
\end{array}\right],\left[\begin{array}{cccccc}
b & b & u & b & b & b \\
& d & d & & c & d
\end{array}\right],\left[\begin{array}{ccc}
c & c & u \\
d & d & d
\end{array}\right],\left[\begin{array}{ccccccc}
\sqcup & \sqcup & \sqcup & d & d & d & \sqcup \\
e & e & e & e & v & e & e
\end{array}\right]\right\}
$$


e a $v$-estrela $D(\mathrm{~A}, 4)$. Temos então

$$
\begin{aligned}
& \begin{array}{l|llll} 
& 0 & 1 & 2 & 3 \\
\hline 1 & 1 & 0 & 0 & 0
\end{array} \quad \begin{array}{l|llll}
0 & 1 & 2 & 3 \\
\hline 1 & 0 & 0 & 0 & 0 \\
2 & 1 & 0 & 0 & 0
\end{array}
\end{aligned}
$$

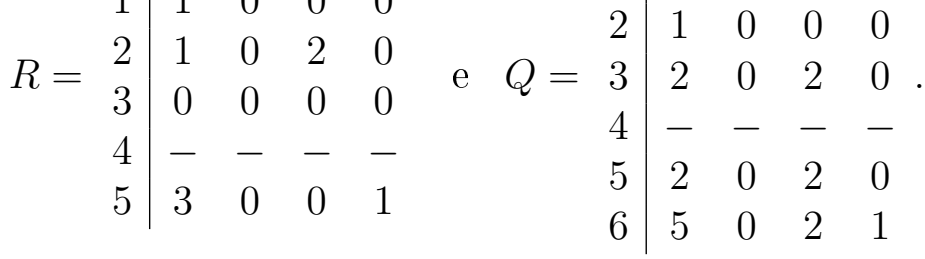

Ponha

$$
s_{c}^{\prime \prime}=\underbrace{\cdots \cdots \sqcup}_{Q(k+1,0)} s_{c}(1) \underbrace{\cdots \cdots \sqcup}_{Q(k+1,1)} s_{c}(2) \ldots s_{c}\left(\left|s_{c}\right|-1\right) \underbrace{\cdots}_{Q\left(k+1,\left|s_{c}\right|-1\right)} s_{c}\left(\left|s_{c}\right|\right) \underbrace{\cdots \cdots}_{Q\left(k+1,\left|s_{c}\right|\right)} .
$$

Seja $C(h, 0)=0, C\left(h,\left|s_{c}(j)\right|+1\right)=\left|s_{h}^{\prime}\right|+1$ e $C(h, j)$ o índice do símbolo em $s_{h}^{\prime}, h \neq c$, que está alinhado com $s_{c}(j)$ para $j=1, \ldots, s_{h}^{\prime}\left(\left|s_{c}(j)\right|\right)$. Definimos, para $j=0, \ldots, s_{h}^{\prime}\left(\left|s_{c}(j)\right|\right)$,

$$
P(h, j)=\underbrace{\underbrace{\cdots} \sqcup}_{Q(h, j)} \underbrace{s_{h}^{\prime}(C(h, j)+1 \ldots C(h, j+1)-1)}_{R(h, j)} \underbrace{\underbrace{\cdots} \sqcup}_{Q(k+1, j)-Q(h, j)-R(h, j)} .
$$

No nosso exemplo,

$$
C=\begin{array}{c|ccccc} 
& 0 & 1 & 2 & 3 & 4 \\
\hline 1 & 0 & 2 & 3 & 4 & 5 \\
2 & 0 & 2 & 3 & 6 & 7 \\
3 & 0 & 1 & 2 & 3 & 4 \\
4 & - & - & - & - & - \\
5 & 0 & 4 & 5 & 6 & 8
\end{array} .
$$

Ponha agora para todo $h \neq c$

$$
s_{h}^{\prime \prime}=P(h, 0) \cdot s_{h}^{\prime}(C(h, 1)) \cdot P(h, 1) \cdot s_{h}^{\prime}(C(h, 2)) \cdot \ldots \cdot s_{h}^{\prime}\left(C\left(h,\left|s_{c}\right|\right)\right) \cdot P\left(h,\left|s_{c}\right|\right) .
$$

O Algoritmo AlinhaCompativel $(D(\mathrm{~A}, c))$ devolve então o alinhamento $\left[s_{1}^{\prime \prime}, \ldots, s_{k}^{\prime \prime}\right]$. Assim, obtemos o alinhamento

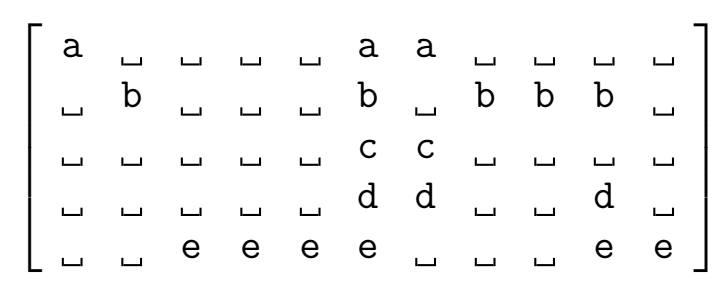

do nosso exemplo.

Por construção do algoritmo, temos os seguintes resultados.

Fato 92. Seja $A=\left[s_{1}^{\prime}, \ldots, s_{k}^{\prime}\right]$ devolvido por AlinhaCompativel $\left(D\left(\mathrm{~A}^{c}, c\right)\right)$. Então, para cada $j$, se $s_{c}^{\prime}(j)=\_$, existe um único inteiro $h, 1 \leq h \leq k$ tal que $s_{h}^{\prime}(j) \neq \sim^{\circ}$. Além disso, sendo $\left(D\left(\mathrm{~A}^{c}, c\right)\right)$ um $c$-desdobramento, se $s_{h}^{\prime}(j) \neq \neq_{\sqcup}$ e $s_{c}^{\prime}(j) \neq \neq_{\sqcup}$, então $\gamma_{s_{h}^{\prime}(j) \rightarrow \smile}>\gamma_{s_{c}^{\prime}(j) \rightarrow s_{h}^{\prime}(j)}$.

Seja $Q_{\max }=\max _{\mathrm{a} \in \Sigma}\left\{\gamma_{\mathrm{a} \rightarrow \boldsymbol{\sqcup}}, \gamma_{\boldsymbol{\iota} \rightarrow \mathrm{a}}\right\}$ e considere o seguinte resultado.

Proposição 93. Considere que $A=\left[s_{1}^{\prime}, \ldots, s_{k}^{\prime}\right]$ é um alinhamento devolvido pelo Algoritmo AlinhaCompativel $\left(D\left(\mathrm{~A}^{c}, c\right)\right)$. Se $\gamma \in \mathbb{M}^{\mathrm{A}}$, então, para $h \neq c$ e $i \neq c$, temos que 
(i) $\gamma_{s_{h}^{\prime}(j) \rightarrow s_{i}^{\prime}(j)} \leq \gamma_{s_{h}^{\prime}(j) \rightarrow s_{c}^{\prime}(j)}+\gamma_{s_{c}^{\prime}(j) \rightarrow s_{i}^{\prime}(j)}$ para cada $j=1, \ldots,|A| \mathrm{e}$

(ii) $v \mathrm{~N}_{\gamma}\left(A_{\{h, i\}}\right) \leq 2 Q_{\max }$.

Prova. Vamos mostrar que vale $(i)$. Seja $j$ um inteiro arbitrário e suponha que $\gamma \in \mathbb{M}^{\mathrm{A}}$. Verificamos a validade do item analisando os possíveis valores de $s_{h}^{\prime}(j), s_{i}^{\prime}(j)$ e $s_{c}^{\prime}(j)$.

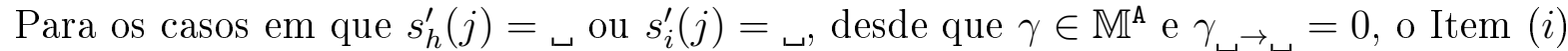
pode ser verificado usando as propriedades de $\mathbb{M}^{\mathrm{A}}$ na Tabela 4.2 do Capítulo 2 considerando os diversos casos possíveis em que acontece $s_{h}^{\prime}(j)={ }$ ou $s_{i}^{\prime}(j)=\_$. Logo, para finalizar a prova do item, assumimos que $s_{h}^{\prime}(j) \neq \_$e $s_{i}^{\prime}(j) \neq \_$. Segue da contrapositiva do Fato 92 que

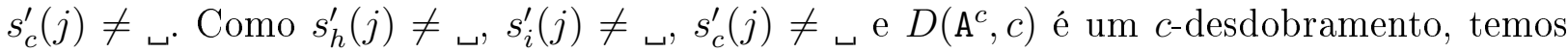
que $\gamma_{s_{h}^{\prime}(j) \rightarrow_{\sqcup}}>\gamma_{s_{c}^{\prime}(j) \rightarrow s_{h}^{\prime}(j)}$ e $\gamma_{s_{i}^{\prime}(j) \rightarrow \sqcup}>\gamma_{s_{c}^{\prime}(j) \rightarrow s_{i}^{\prime}(j)}$.

Como $\gamma_{s_{h}^{\prime}(j) \rightarrow_{\sqcup}}>\gamma_{s_{c}^{\prime}(j) \rightarrow s_{h}^{\prime}(j)}$, pelo Fato 89 temos que $\gamma_{s_{h}^{\prime}(j) \rightarrow_{\sqcup}}>\gamma_{s_{h}^{\prime}(j) \rightarrow s_{c}^{\prime}(j)}$. Desde que $\gamma_{s_{i}^{\prime}(j) \rightarrow \sqcup}>\gamma_{s_{c}^{\prime}(j) \rightarrow s_{i}^{\prime}(j)}$, segue da definição de $\mathbb{M}^{\mathrm{A}}$ que $\gamma_{\sqcup^{\prime} \rightarrow s_{i}^{\prime}(j)}=\gamma_{s_{i}^{\prime}(j) \rightarrow \sqcup}>\gamma_{s_{c}^{\prime}(j) \rightarrow s_{i}^{\prime}(j)}$. Como $\gamma_{s_{h}^{\prime}(j) \rightarrow \sqcup}>\gamma_{s_{h}^{\prime}(j) \rightarrow s_{c}^{\prime}(j)}$ e $\gamma_{\sqcup \rightarrow s_{i}^{\prime}(j)}>\gamma_{s_{c}^{\prime}(j) \rightarrow s_{i}^{\prime}(j)}$, temos que $\gamma_{s_{h}^{\prime}(j) \rightarrow \sqcup}+\gamma_{\sqcup \rightarrow s_{i}^{\prime}(j)}>\gamma_{s_{h}^{\prime}(j) \rightarrow s_{c}^{\prime}(j)}+$ $\gamma_{s_{c}^{\prime}(j) \rightarrow s_{i}^{\prime}(j)}$. Segue da definição de $\mathbb{M}^{\mathrm{A}}$ que $\gamma_{s_{h}^{\prime}(j) \rightarrow s_{i}^{\prime}(j)} \leq \gamma_{s_{h}^{\prime}(j) \rightarrow s_{c}^{\prime}(j)}+\gamma_{s_{c}^{\prime}(j) \rightarrow s_{i}^{\prime}(j)}$, finalizando a prova do Item $(i)$.

Vamos agora mostrar o Item $(i i)$. Suponha sem perda de generalidade que $h<i$, vamos primeiramente provar que, para qualquer inteiro $j, \gamma_{s_{h}^{\prime}(j) \rightarrow s_{i}^{\prime}(j)} \leq 2 Q_{\max }$. Se $s_{h}^{\prime}(j)=\longleftarrow$ ou $s_{i}^{\prime}(j)={ }_{\iota}$, então, como $\gamma_{\iota^{\prime}}=0$ e $\gamma \in \mathbb{M}^{\mathrm{A}}$, considerando os diversos casos possíveis em que acontece $s_{h}^{\prime}(j)={ }_{\iota}$ ou $s_{i}^{\prime}(j)={ }_{\iota}$, temos que vale $\gamma_{s_{h}^{\prime}(j) \rightarrow s_{i}^{\prime}(j)} \leq Q_{\max } \leq 2 Q_{\max }$ e o item está provado. Assumimos então que $s_{h}^{\prime}(j) \neq \_$e $s_{i}^{\prime}(j) \neq \neq^{\prime}$. Segue do Fato 92 que $s_{c}^{\prime}(j) \neq \sqcup$

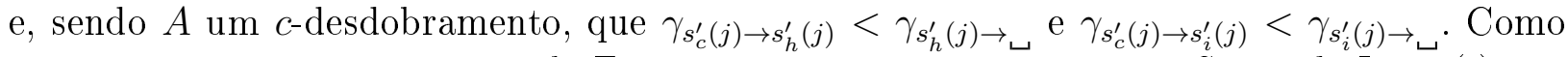
$\gamma_{s_{c}^{\prime}(j) \rightarrow s_{h}^{\prime}(j)}<\gamma_{s_{h}^{\prime}(j) \rightarrow \sqcup}$, temos pelo Fato 89 que $\gamma_{s_{h}^{\prime}(j) \rightarrow s_{c}^{\prime}(j)}<\gamma_{s_{h}^{\prime}(j) \rightarrow \sqcup}$. Segue do Item $(i)$ que $\gamma_{s_{h}^{\prime}(j) \rightarrow s_{i}^{\prime}(j)} \leq \gamma_{s_{h}^{\prime}(j) \rightarrow s_{c}^{\prime}(j)}+\gamma_{s_{c}^{\prime}(j) \rightarrow s_{i}^{\prime}(j)}<\gamma_{s_{h}^{\prime}(j) \rightarrow_{\sqcup}}+\gamma_{s_{i}^{\prime}(j) \rightarrow_{\sqcup}} \leq Q_{\max }+Q_{\max }=2 Q_{\text {max }}$. Segue que $v \mathrm{~N}_{\gamma}\left(A_{\{h, i\}}\right)=v \mathrm{~A}_{\gamma}\left(A_{\{h, i\}}\right) /\left|A_{\{h, i\}}\right| \leq 2 Q_{\max }\left|A_{\{h, i\}}\right| /\left|A_{\{h, i\}}\right|=2 Q_{\max }\left|A_{\{h, i\}}\right|$, finalizando a prova do Item $(i i)$.

Lema 94. Seja $A=\left[s_{1}^{\prime}, \ldots, s_{k}^{\prime}\right]$ devolvido por AlinhaCompativel $\left(D\left(\mathrm{~A}^{c}, c\right)\right)$. Então,

- se $\gamma \in \mathbb{M}^{\mathrm{A}}$, então $v \mathrm{~A}_{\gamma}\left(A_{\{h, i\}}\right) \leq v \mathrm{~A}_{\gamma}\left(A_{\{h, c\}}\right)+v \mathrm{~A}_{\gamma}\left(A_{\{c, i\}}\right)$ e

- se $\gamma \in \mathbb{M}^{\mathbb{N}}$, então $v \mathrm{~N}_{\gamma}\left(A_{\{h, i\}}\right) \leq 2 \cdot\left(v \mathrm{~N}_{\gamma}\left(A_{\{h, c\}}\right)+v \mathrm{~N}_{\gamma}\left(A_{\{c, i\}}\right)\right)$

para cada $h<i, h \neq c, i \neq c$.

Prova. Sejam $h, i$ inteiros arbitrários e $X=\left\{j: s_{c}^{\prime}(j) \neq{ }_{\sqcup}\right.$ e $\left.s_{h}^{\prime}(j)=s_{i}^{\prime}(j)=_{\sqcup}\right\}$ e suponha que $\gamma \in \mathbb{M}^{A}$. Segue que

$$
\begin{aligned}
& v \mathrm{~A}_{\gamma}\left(A_{\{h, i\}}\right)+\sum_{j \in X}\left(\gamma_{\sqcup \rightarrow s_{c}^{\prime}(j)}+\gamma_{s_{c}^{\prime}(j) \rightarrow \sqcup}\right)=\sum_{j \notin X} \gamma_{s_{h}^{\prime}(j) \rightarrow s_{i}^{\prime}(j)}+\sum_{j \in X}\left(\gamma_{\sqcup \rightarrow s_{c}^{\prime}(j)}+\gamma_{s_{c}^{\prime}(j) \rightarrow \smile}\right) \\
& \leq \sum_{j \notin X}\left(\gamma_{s_{h}^{\prime}(j) \rightarrow s_{c}^{\prime}(j)}+\gamma_{s_{c}^{\prime}(j) \rightarrow s_{i}^{\prime}(j)}\right)+ \\
& \sum_{j \in X}\left(\gamma_{\sqcup \rightarrow s_{c}^{\prime}(j)}+\gamma_{s_{c}^{\prime}(j) \rightarrow}\right) \\
& =v \mathrm{~A}_{\gamma}\left(A_{\{h, c\}}\right)+v \mathrm{~A}_{\gamma}\left(A_{\{c, i\}}\right) \text {, }
\end{aligned}
$$

onde a desigualdade vale pela Proposição 93. Portanto,

$$
v \mathrm{~A}_{\gamma}\left(A_{\{h, i\}}\right) \leq v \mathrm{~A}_{\gamma}\left(A_{\{h, c\}}\right)+v \mathrm{~A}_{\gamma}\left(A_{\{c, i\}}\right)-\sum_{j \in X}\left(\gamma_{\sqcup \rightarrow s_{c}^{\prime}(j)}+\gamma_{s_{c}^{\prime}(j) \rightarrow \sqcup}\right)
$$




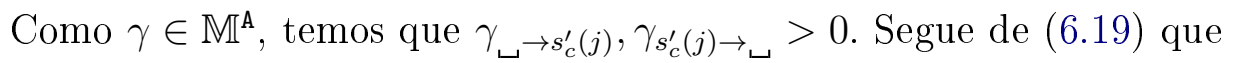

$$
\begin{aligned}
v \mathrm{~A}_{\gamma}\left(A_{\{h, i\}}\right) & \leq v \mathrm{~A}_{\gamma}\left(A_{\{h, c\}}\right)+v \mathrm{~A}_{\gamma}\left(A_{\{c, i\}}\right)-\sum_{j \in X}\left(\gamma_{\sqcup \rightarrow s_{c}^{\prime}(j)}+\gamma_{s_{c}^{\prime}(j) \rightarrow}\right) \\
& \leq v \mathrm{~A}_{\gamma}\left(A_{\{h, c\}}\right)+v \mathrm{~A}_{\gamma}\left(A_{\{c, i\}}\right)
\end{aligned}
$$

e o primeiro item da proposição está provado.

Suponha agora que $\gamma \in \mathbb{M}^{\mathbb{N}}$. Segue da definição de $\mathbb{M}^{\mathbb{N}}$ que

$$
Q_{\max }=\max _{\sigma \in \Sigma}\left\{\gamma_{\sigma \rightarrow \sqcup}, \gamma_{\sqcup \rightarrow \sigma}\right\}=\max _{\sigma \in \Sigma}\left\{\gamma_{\sigma \rightarrow_{\sqcup}}\right\} \leq 2 \gamma_{s_{c}^{\prime}(j) \rightarrow_{\sqcup}}=\gamma_{\sqcup \rightarrow s_{c}^{\prime}(j)}+\gamma_{s_{c}^{\prime}(j) \rightarrow_{\sqcup}} .
$$

para todo $j$. Além disso,

$$
\left|A_{\{h, c\}}\right| \leq\left|A_{\{h, i, c\}}\right|, \quad\left|A_{\{c, i\}}\right| \leq\left|A_{\{h, i, c\}}\right| \quad \text { e } \quad\left|A_{\{h, i\}}\right|=\left|A_{\{h, i, c\}}\right|-|X| .
$$

Segue daí que

$$
\begin{aligned}
v \mathrm{~N}_{\gamma}\left(A_{\{h, i\}}\right)= & \frac{v \mathrm{~A}_{\gamma}\left(A_{\{h, i\}}\right)}{\left|A_{\{h, i\}}\right|} \\
\leq & \frac{v \mathrm{~A}_{\gamma}\left(A_{\{h, i\}}\right)+2 \cdot Q_{\max }|X|}{\left|A_{\{h, i\}}\right|+|X|} \leq 2 \cdot \frac{v \mathrm{~A}_{\gamma}\left(A_{\{h, i\}}\right)+Q_{\max }|X|}{\left|A_{\{h, i\}}\right|+|X|} \\
\leq & 2 \cdot\left(\frac{v \mathrm{~A}_{\gamma}\left(A_{\{h, c\}}\right)+v \mathrm{~A}_{\gamma}\left(A_{\{c, i\}}\right)}{\left|A_{\{h, i, c\}}\right|-|X|+|X|}-\right. \\
& \left.\frac{\sum_{j \in X}\left(\gamma_{\sqcup \rightarrow s_{c}^{\prime}(j)}+\gamma_{s_{c}^{\prime}(j) \rightarrow}\right)+Q_{\max }|X|}{\left|A_{\{h, i, c\}}\right|-|X|+|X|}\right) \\
\leq & 2 \cdot \frac{v \mathrm{~A}_{\gamma}\left(A_{\{h, c\}}\right)+v \mathrm{~A}_{\gamma}\left(A_{\{c, i\}}\right)-Q_{\max }|X|+Q_{\max }|X|}{\left|A_{\{h, i, c\}}\right|-|X|+|X|} \\
= & 2 \cdot \frac{v \mathrm{~A}_{\gamma}\left(A_{\{h, c\}}\right)+v \mathrm{~A}_{\gamma}\left(A_{\{c, i\}}\right)}{\left|A_{\{h, i, c\}}\right|}=\frac{v \mathrm{~A}_{\gamma}\left(A_{\{h, c\}}\right)}{\left|A_{\{h, i, c\}}\right|}+\frac{v \mathrm{~A}_{\gamma}\left(A_{\{c, i\}}\right)}{\left|A_{\{h, i, c\}}\right|} \\
\leq & 2 \cdot \frac{v \mathrm{~A}_{\gamma}\left(A_{\{h, c\}}\right)}{\left|A_{\{h, c\}}\right|}+\frac{v \mathrm{~A}_{\gamma}\left(A_{\{c, i\}}\right)}{\left|A_{\{c, i\}}\right|}=2 \cdot\left(v \mathrm{~N}_{\gamma}\left(A_{\{h, c\}}\right)+v \mathrm{~N}_{\gamma}\left(A_{\{c, i\}}\right)\right) .
\end{aligned}
$$

onde a primeira desigualdade de (6.20) segue da Proposição 93 (dado que $\mathbb{M}^{\mathbb{N}} \subseteq \mathbb{M}^{\mathbb{A}}$ ) e do Fato 53, e a segunda desigualdade segue de $v \mathrm{~A}_{\gamma}\left(A_{\{h, i\}}\right) \geq 0$ (dado que toda entrada de $\gamma$ é maior ou igual a zero); (6.21) segue de (6.19) e de $\left|A_{\{h, i\}}\right|=\left|A_{\{h, i, c\}}\right|-|X|$, e (6.22) segue da definição de $Q_{\max }$. Portanto, se $\gamma \in \mathbb{M}^{\mathbb{N}}$, a proposição também está provada.

Para calcular o tempo gasto para determinar um alinhamento compatível, suponha que cada sequência de $S$ possui comprimento $n$. Note que cada linha da matriz $R$ pode ser computada consultando uma única vez cada coluna de um alinhamento $A_{\{h, c\}}$ que possui comprimento máximo $2 n$. Portanto, a matriz $R$ pode ser computada gastando tempo $O(k n)$. Similarmente as matrizes $Q$ e $C$ gastam tempo $O(k n)$ para serem computadas. Depois, para cada elemento do alinhamento obtido, consultamos uma quantidade constante de entradas das matrizes $R, Q$ e $C$ o que implica que o tempo gasto é proporcional à quantidade de elementos de $A$ que é $O\left(k^{2} n\right)$. Portanto, AlinhaCompativel gasta tempo $O\left(k^{2} n\right)$.

\subsubsection{Algoritmo}

Teorema 95. O Algoritmo 8 está correto. Ademais, sendo $O\left(n^{2}\right)$ um limitante superior 


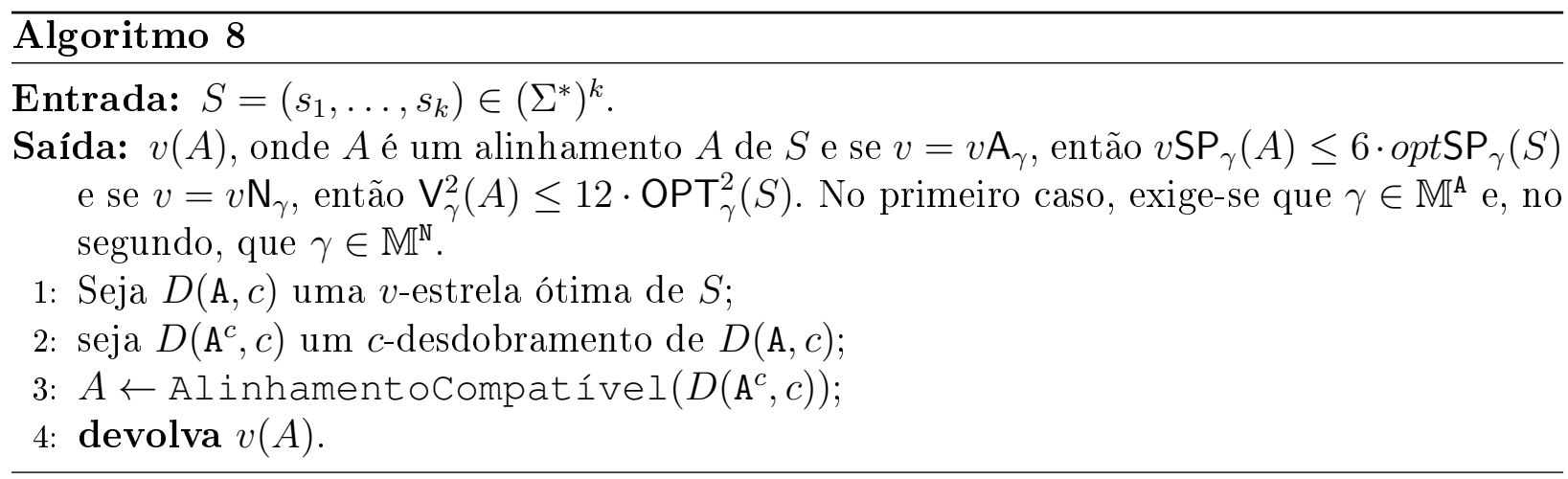

de tempo para encontrar a $v \mathrm{~A}_{\gamma}$-pontuação de um alinhamento A-ótimo de duas sequências de comprimento $n$ e seu respectivo alinhamento quando todas as sequências de $S$ têm comprimento $n$, temos que o tempo gasto pelo algoritmo é $O\left(k^{2} n^{2}\right)$.

Prova. Claramente o valor devolvido pelo Algoritmo 8 é a pontuação de um alinhamento de $S$. Vamos mostrar então que o fator de aproximação é o esperado.

Suponha primeiro que $v=v \mathrm{~A}_{\gamma}$ e $\gamma \in \mathbb{M}^{\mathrm{A}}$. Então,

$$
\begin{aligned}
v \mathrm{SP}_{\gamma}(A) & =\sum_{h=1}^{k-1} \sum_{i=h+1}^{k} v \mathrm{~A}_{\gamma}\left(A_{\{h, i\}}\right) \\
& \leq \sum_{h=1}^{k-1} \sum_{i=h+1}^{k}\left(v \mathrm{~A}_{\gamma}\left(A_{\{h, c\}}\right)+v \mathrm{~A}_{\gamma}\left(A_{\{c, i\}}\right) \leq 6 \cdot o p t \mathrm{SP}_{\gamma}(S),\right.
\end{aligned}
$$

onde a primeira desigualdade segue do Lema 94 e a segunda segue do Lema 91. Portanto, o fator de aproximação é o esperado para este caso.

Suponha agora que $v=v \mathrm{~N}_{\gamma}$ e $\gamma \in \mathbb{M}^{\mathrm{N}}$. Então, nesse caso,

$$
\begin{aligned}
\mathrm{V}_{\gamma}^{2}(A) & =\sum_{h=1}^{k-1} \sum_{i=h+1}^{k} v \mathrm{~N}_{\gamma}\left(A_{\{h, i\}}\right) \\
& \leq \sum_{h=1}^{k-1} \sum_{i=h+1}^{k} 2\left(v \mathrm{~N}_{\gamma}\left(A_{\{h, c\}}\right)+v \mathrm{~N}_{\gamma}\left(A_{\{c, i\}}\right)\right)=2 \cdot \sum_{h=1}^{k-1} \sum_{i=h+1}^{k}\left(v \mathrm{~N}_{\gamma}\left(A_{\{h, c\}}\right)+v \mathrm{~N}_{\gamma}\left(A_{\{c, i\}}\right)\right) \\
& \leq 2 \cdot 6 \cdot \mathrm{OPT}_{\gamma}^{2}(S)=12 \cdot \mathrm{OPT}_{\gamma}^{2}(S),
\end{aligned}
$$

onde, similarmente, a primeira desigualdade segue do Lema 94 e a segunda segue do Lema 91 e, novamente, o fator de aproximação está correto também para este caso.

O tempo gasto para encontrar uma $v$-estrela ótima é o tempo para calcular as pontuações dos alinhamentos dois-a-dois de $S$ que é $\left(\begin{array}{c}k \\ 2\end{array}\right) O\left(n^{2}\right)$ mais o tempo para determinar os $(k-1)$ alinhamentos da estrela ótima que é $(k-1) O\left(n^{2}\right)$. Portanto, o tempo gasto para calcular a Linha 1 é $\left(\begin{array}{l}k \\ 2\end{array}\right) O\left(n^{2}\right)+(k-1) O\left(n^{2}\right)=O\left(k^{2} n^{2}\right)$.

O tempo gasto para calcular as Linhas 2 e 3 é $O\left(k^{2} n\right)$ cada e o da Linha 4 é $O\left(k^{3} n\right)$, pois temos que computar o custo de $\left(\begin{array}{l}k \\ 2\end{array}\right) O\left(k^{2}\right)$ alinhamentos de duas sequências de comprimento $O(k n)$. Portanto, o tempo total gasto pelo algoritmo é

$$
O\left(k^{2} n^{2}\right)+O\left(k^{2} n\right)+O\left(k^{2} n\right)+O\left(k^{3} n\right)=O\left(k^{2} n^{2}+k^{3} n\right) .
$$


Finalizamos o capítulo, resumindo na Tabela 6.1 os resultados de nossa pesquisa.

\begin{tabular}{c|c|c|c} 
Problema & Tempo - AE & NP-completude & Tempo - AA \\
\hline \hline AVS & - & - & $O\left(k^{2} n^{2}+k^{3} n\right)$ \\
\hline AVSN-1 & $O\left(2^{k} k^{3}(n+1)^{k+1}\right)$ & \multirow{2}{*}{ Sim } & $O\left(k^{2} n^{2}+k^{3} n\right)$ \\
\hline AVSN-2 & $O\left(\left(1+\frac{1}{2 n+1}\right)^{k}(2 n+1)^{k^{2}} k^{2}\right)$ & & - \\
\hline AVSN-3 & $O\left(2^{k} k^{4}(n+1)^{k+1}\right)$ & & - \\
\hline \hline
\end{tabular}

Tabela 6.1: Consideramos no estudo da complexidade que a quantidade de sequências é $k$ e que cada uma das sequências possui comprimento n; Tempo - $\mathbf{A E}$ é a coluna que contém o tempo gasto de cada algoritmo exato aqui descrito; Tempo - AA é a coluna que contém o tempo gasto de cada algoritmo aproximado aqui descrito: para o Problema $\boldsymbol{A V S}$, o algoritmo é uma 6-aproximação e para o Problema $\boldsymbol{A} \boldsymbol{V} \boldsymbol{S N}$-2, o algoritmo é uma 12-aproximação quando a matriz de pontuação está em $\mathbb{M}^{\mathrm{N}}$. 


\section{Capítulo 7}

\section{Conclusão}

Neste capítulo fazemos uma breve revisão do que foi estudado neste doutorado, sugerindo trabalhos futuros.

No Capítulo 2 apresentamos os conceitos básicos desenvolvidos durante a tese e alguns resultados encontrados na literatura. Ademais, mostramos um algoritmo inédito para o Problema APSN. Esse algoritmo não representa uma contribuição importante além desta tese pois, pelo menos assintoticamente, não é mais rápido do que o melhor algoritmo para o problema e porque seu uso é restrito, pois somente pode ser usados se a matriz de pontuação possui custo uniforme de inserção e remoção.

No Capítulo 3 apresentamos uma estrutura capaz de representar uma sequência de operações de edição que transformam uma sequência na outra. Para $a, b \in \Sigma^{*}$, descrevemos um algoritmo que computa a pontuação de uma sequência de operações de edição que transforma a em $\mathrm{b}$ e que possui pontuação mínima.

Como consequência dos resultados obtidos no Capítulo 4 podemos caracterizar as matrizes de pontuação $\gamma$ que induzem vários tipos de funções (pramétrica, semimétrica, quasimétrica, etc) nas sequências. Fazemos isto para os critérios $v \mathrm{~A}_{\gamma}, v \mathrm{~N}_{\gamma}$ e $v \mathrm{E}_{\gamma}$.

No Capítulo 5 determinamos, para uma classe ampla de matrizes de pontuação, as condições necessárias e suficientes para que duas matrizes de pontuação sejam equivalentes. Neste texto, consideramos que as matrizes de pontuação $\gamma$ e $\delta$ são equivalentes se, para quaisquer alinhamentos $A$ e $B$ de duas sequências, $v \mathrm{~A}_{\gamma}(A) \leq v \mathrm{~A}_{\gamma}(B)$ se e somente se $v \mathrm{~A}_{\delta}(A) \leq v \mathrm{~A}_{\delta}(B)$. Entretanto, em geral não estamos interessados em quaisquer alinhamentos mas sim em alinhamentos A-ótimos. Seria interessante caracterizar matrizes de pontuação $\gamma$ e $\delta$ que tenham a seguinte propriedade: $o p t \mathrm{~A}_{\gamma}(s, t)=v \mathrm{~A}_{\gamma}(A)$ se e somente se opt $\mathrm{A}_{\delta}(s, t)=v \mathrm{~A}_{\delta}(A)$.

No Capítulo 6 desta tese, usando o critério $v \mathrm{~A}_{\gamma}$ para pontuar duas sequências, descrevemos um algoritmo que é uma 6-aproximação para o Problema AVS quando a matriz de pontuação está em $\mathbb{M}^{\mathrm{A}}$. Trocando o critério $v \mathrm{~A}_{\gamma}$ por $v \mathrm{~N}_{\gamma}$, se $\gamma \in \mathbb{M}^{\mathbb{N}}$, esse algoritmo é uma 12-aproximação para a versão de otimização do Problema AVSN-2. Bafna, Lawler e Pevzner [BLP97] descrevem um algoritmo para o Problema AVS quando $\gamma \in \mathbb{M}^{\mathrm{C}}$ que é uma $l / k$-aproximação e que é polinomial em $n$ e $k$ para um $l$ fixo. Seria interessante investigar se o mesmo algoritmo pode ser adaptado para os problemas AVS quando $\gamma \in \mathbb{M}^{\mathbb{A}}$ e para a versão de otimização de AVSN-2 quando $\gamma \in \mathbb{M}^{\mathrm{N}}$.

Um esquema de aproximação em tempo polinomial - polynomial time approximation schemes (PTAS) consiste em um algoritmo que recebe como entrada uma instância de um problema de otimização e um número $\varepsilon>0$ e que é uma $(1+\varepsilon)$-aproximação e, em tempo polinomial no tamanho da entrada, devolve uma solução. A classe MAX-SNP-difícil é a classe dos problemas que não possuem PTAS a menos que P = NP. Just [Jus01] mostrou que o Problema AVS é MAX-SNP-difícil quando a matriz de pontuação é uma supermatriz 
de

\begin{tabular}{c|cccc} 
& $\mathrm{a}$ & $\mathrm{b}$ & $\mathrm{c}$ & $\mathbf{c}$ \\
\hline $\mathrm{a}$ & 0 & 1 & 0 & 2 \\
$\mathrm{~b}$ & 1 & 0 & 0 & 2 \\
$\mathrm{c}$ & 0 & 0 & 0 & 2 \\
$\mathrm{c}$ & 2 & 2 & 2 &
\end{tabular}.

Nesta tese mostramos que, para cada $\eta$, o Problema AVSN- $\eta$ é NP-completo. O algoritmo de aproximação descrito para AVSN-2 mostra que ele pode ser aproximado por uma constante. Seria importante verificar se existem algoritmos de aproximação para os problemas AVSN-1 e AVSN-3 e, para cada $\eta$, se o Problema AVSN- $\eta$ é MAX-SNP-difícil. 


\section{Referências Bibliográficas}

[AE00] A.N. Arslan e Ö. Egecioglu. Efficient algorithms for normalized edit distance. Journal of Discrete Algorithms, 1(1):3-20, 2000. 18, 28

[AG97] A. Apostolico e Z. Galil. Pattern Matching Algorithms. Oxford University Press, USA, 1997. 1

[AP90] A. V. Arkhangel'skiǐ e L. S. Pontryagin. General Topology I: Basic Concepts and Constructions, Dimension Theory. Springer-Verlag, Berlin, 1990. 43

[AS06] E. Araújo e J. Soares. Scoring matrices that induce metrics on sequences. Em José R. Correa, Alejandro Hevia, e Marcos A. Kiwi, editors, LATIN, volume 3887 of Lecture Notes in Computer Science, páginas 68-79. Springer, 2006. 2, 4, 5

[BLP97] V. Bafna, E.L. Lawler, e P.A. Pevzner. Approximation algorithms for multiple sequence alignment. Theoretical Computer Science, 182(1-2):233-244, 1997. 109

[Bri03] R. Brito. Alinhamento de sequências biológicas. Dissertação de Mestrado, Instituto de Matemática e Estatística - IME - USP, 2003. 30

[BV01] P. Bonizzoni e G.D. Vedova. The complexity of multiple sequence alignment with SP-score that is a metric. Theoretical Computer Science, 259(1-2):63-79, 2001. 35

[Chv80] V. Chvátal. Recognizing intersection patterns. Em Annals Discrete Mathematics, volume 8, páginas 249-251. North Holland Publishing Company, 1980. 91

[CL88] H. Carrillo e D. Lipman. The multiple sequence alignment problem in biology. SIAM Journal on Applied Mathematics, 48(5):1073-1082, 1988. 30

[CLZU02] M. Crochemore, G.M. Landau, e M. Ziv-Ukelson. A sub-quadratic sequence alignment algorithm for unrestricted cost matrices. Em Proceedings of the thirteenth annual ACM-SIAM symposium on Discrete algorithms, SODA '02, páginas 679-688, Philadelphia, PA, USA, 2002. Society for Industrial and Applied Mathematics. 13, 28

[Eli06] I. Elias. Settling the intractability of multiple alignment. Journal of Computational Biology, 13(7):1323-1339, September 2006. 35

[Flo62] R.W. Floyd. Algorithm 97: shortest path. Communications of the ACM, 5(6):345, 1962. 41

$\left[\mathrm{GPB}^{+} 02\right]$ G. Gutin, A. Punnen, A. Barvinok, E. Gimadi, e A. Serdyukov. The Traveling Salesman Problem and Its Variations. Springer, 2002. 9 
[Gus93] D. Gusfield. Efficient methods for multiple sequence alignment with guaranteed error bounds. Bulletin of Mathematical Biology, 55(1):141-154, 1993. 98

[Jus01] W. Just. Computational complexity of multiple sequence alignment with SPscore. Journal of computational biology, 8(6):615-623, 2001. 35, 109

[Kim68] Y. Kim. Pseudo quasi metric spaces. Proceedings of the Japan Academy, 1968. 43

[Lev66] V.I. Levenshtein. Binary codes capable of correcting deletions, insertions and reversals. Soviet Physics Doklady, 10(8):707-710, 1966. 2

[MP80] W.J. Masek e M.S. Paterson. A faster algorithm computing string edit distances. Journal of Computer and System sciences, 20(1):18-31, 1980. 13, 28

[MV93] A. Marzal e E. Vidal. Computation of normalized edit distance and applications. IEEE Transactions on Pattern Analysis and Machine Intelligence, 15(9):926-932, 1993. 1, 3, 13, 15, 28, 44

[Nav06] G. Navarro. Comunicação pessoal, 2006. 2

[NW70] S.B. Needleman e C.D. Wunsch. A general method applicable to the search for similarities in the amino acid sequence of two proteins. Journal of molecular biology, 48(3):443-453, 1970. 9, 11, 28

[RU81] K. Räihä e E. Ukkonen. The shortest common supersequence problem over binary alphabet is NP-complete. Theoretical Computer Science, 16:187-198, 1981. 31

[Sel74] P.H. Sellers. On the theory and computation of evolutionary distances. SIAM Journal on Applied Mathematics, 26(4):787-793, 1974. 2, 44

[SM97] J.C. Setubal e J. Meidanis. Introduction to computational molecular biology. PWS Publishing Company, 1997. 1, 73

[SS70] L.A. Steen e J.A. Seebach. Counterexamples in Topology. Holt, Rinehart and Winston New York, 1970. 43

[VMA95] E. Vidal, A. Marzal, e P. Aibar. Fast computation of normalized edit distances. Pattern Analysis and Machine Intelligence, IEEE Transactions on, 17(9):899902, 1995. 17, 28

[War62] S. Warshall. A theorem on boolean matrices. Journal of the ACM (JACM), 9(1):11-12, 1962. 41

[Wil31a] W.A. Wilson. On quasi-metric spaces. American Journal of Mathematics, $53(3): 675-684,1931.43$

[Wil31b] W.A. Wilson. On semi-metric spaces. American Journal of Mathematics, 53(2):361-373, 1931. 43

[WJ94] L. Wang e T. Jiang. On the complexity of multiple sequence alignment. Journal of computational biology, 1(4):337-348, 1994. 30, 35

[YB07] L. Yujian e L. Bo. A normalized Levenshtein distance metric. IEEE transactions on pattern analysis and machine intelligence, 29(6):1091-1095, June 2007. 4, 45 


\section{Índice Remissivo}

$k$-vetor, 84

alfabeto, 7

algoritmo

AlinhaCompativel, 102

alinhamento, 7

$V$-ótimo, 99

$v \mathrm{SP}_{\gamma}$-ótimo, 29

v-ótimo, 99

ótimo, 1

$\mathrm{A}(\lambda)$-ótimo, 16

A-ótimo, 10

alinha, 8

alinhados, 8

canônico, 93

cauda, 93

base, 93

coluna

coluna $\sigma, 93$

quantidade de $\sigma, 93$

coluna do, 7

comprimento

base, 93

comprimento do, 8

estendido, 3, 37

ótimo, 38

coluna, 37

comprimento, 37

induzido, 39

induzido, 8

comprimento, 87

matriz dos, 13

N-ótimo, 13

pontuação- $v \mathrm{~N}_{\gamma}, 13$

aproximação

$\alpha$-aproximação, 10

fator de, 10

critério de pontuação

pontuação- $v \mathrm{~A}_{\gamma}, 10$

pontuação- $v \mathrm{~A}_{\gamma}(\lambda), 15$

pontuação- $v \mathrm{E}_{\gamma}, 38$ pontuação- $v \mathrm{SP}_{\gamma}, 28$

critério para a pontuação, 1

desdobramento

$c$-desdobramento de uma $v$-estrela, 100

s-desdobrável, 100

$s$-desdobramento, 100

o conjunto que define, 100

digrafo, 9

arco, 9

aparece, 9

custo, 9

entra, 9

sai, 9

caminho, 9,38

ótimo, 9

comprimento, 9

concatenação de, 9

custo do, 9

ciclo, 9

ciclo negativo, 9

define, 9

simples, 9

custo do, 9

euleriano, 9

vértice, 9

elemento de $\mathbb{A}$

A-ótimo, 21

corresponde ao alinhamento, 21

custo, 21

custo normalizado, 24

define, 21

espaço, 7

estrela

$v$-estrela, 99

ótima, 99

centro, 99

função

$V, 99$

$v \mathrm{~A}_{\gamma}, 2$

opt $\mathrm{A}_{\gamma}, 2$ 
hemimétrica, 43

induzida, 43

Levenshtein, 2

métrica, 43

pramétrica, 43

pseudométrica, 43

quasimétrica, 43

semimétrica, 43

função ótima, 1

$v \mathrm{~A}_{\gamma}(\lambda), 15$

matriz de pontuação, 2,8

custo, 2

equivalentes, 4,73

multiplicação, 74

reflexibilidade, 73

simetria, 73

soma, 74

transitividade, 73

mdc, 77

multiplicação

matriz de pontuação, 74

operação de edição, 2

inserção, 2

remoção, 2

substituição, 2

problema

APS, 10

APSN, 13

AVS, 29

AVSN, 92

AVSd, 30

CME, 38

RACI, 90

RPI, 90

SC-Mín, 31

símbolos, 7

sequência

comprimento, 7

vazia, 7

sequências

supersequência, 30

soma

matriz de pontuação, 74

supermatriz, 30 